REPRESENTATION THEORY

An Electronic Journal of the American Mathematical Society

Volume 14, Pages 510-600 (August 3, 2010)

S 1088-4165(2010)00384-2

\title{
DOUBLE AFFINE HECKE ALGEBRAS AT ROOTS OF UNITY
}

\author{
M. VARAGNOLO AND E. VASSEROT
}

\begin{abstract}
We study double affine Hecke algebras at roots of unity and their relations with deformed Hilbert schemes. In particular their categories of finitely generated modules are derived equivalent to some category of coherent sheaves.
\end{abstract}

\section{INTRODUCTION}

The double affine Hecke algebras, DAHA for short, have been introduced by Cherednik about 15 years ago for the proof of MacDonald conjectures. Their representation theory has been much studied in recent years. In particular, simple representation of the category $\mathcal{O}$ has been classified in $[\mathrm{V}]$ when the parameters are not not roots of one. It is expected that DAHA play some role in modular representations of affine Lie algebras or $p$-adic groups. See [VV2, VV3].

In this paper we study the DAHA when the parameters are roots of unity. We will only consider the type $A_{n}$ case and we will assume that the order of the modular parameter is large enough. Note that the following is already known: the classification of simple modules in type $A_{1}$ when parameters are roots of 1 in C], the classification of simple modules in type $A_{n}$ with generic quantum parameter and modular parameter equal to 1 in $\mathrm{O}$ ] and the classification of simple modules of rational DAHA of type $A_{n}$ in large characteristic in BFG. Here we adapt the technics of the latter work to the DAHA case. The proof is much more complicated in our case. There are several reasons for this.

The first one is that, for DAHA, one must introduce a new ring of quantum differential operators over $G L_{n}$. The usual one, constructed via the Heisenberg double, is not convenient because the adjoint action is not compatible with the multiplication. So one must introduce a twisted version of the Heisenberg double which is, technically, much more complicated.

The second reason is that some standard properties of the rational DAHA (Noetherianity, finiteness of the global dimension, etc.) do not generalize easily. The rational DAHA, which contains large polynomial subalgebras, has a natural finite filtration with a nice associated graded ring. The DAHA does not have such a filtration, basically because the polynomial subalgebras are replaced there by algebras of Laurent polynomials. This problem has another consequence. As we explain below, one of the main goals of our work is to compare the spherical DAHA with the algebra of global section of a sheaf of quantum differential operators on a

Received by the editors June 14, 2006 and, in revised form, January 21, 2009

2000 Mathematics Subject Classification. Primary 17B37; Secondary 16W35, 20 C08. 
smooth variety (the deformed Hilbert scheme). Traditionally such statements are proved as follows:

- one embeds the spherical DAHA into a quantum torus via Dunkl operators,

- one embeds the algebra of quantum differential operators into a quantum torus via a homomorphism "à la Harish-Chandra",

- one compares the two subalgebras using some filtered/graded arguments.

In our case the first step makes sense (see section 3.3), the second one also makes sense (see sections 1.11 and 2.8), but the third step does not make sense (at least to our knowledge). One of the basic tools that we use instead of filtred/graded arguments comes from symplectic geometry. More precisely, we assume that the parameters are roots of unity. So the algebras we must compare have large centers. They are Poisson orders, following the terminology in [BG2]. See section A.10 for details.

One could imagine that the DAHA's representations can be recovered from the representations of the rational DAHA. The geometric construction we give below shows that this cannot be, because DAHA's representations are classified by quasicoherent sheaves over a family of deformed Hilbert schemes which strictly contains the Hilbert schemes entering in the rational case.

Now recall that the proof in $[\mathrm{BFG}$, is based on the following proposition of Bezrukavnikov and Kaledin.

Proposition. Let $k$ be an algebraically closed field. Let $X$ be a smooth connected $k$-variety with trivial canonical class and let $Y$ be an affine variety. Let $f: X \rightarrow Y$ be a proper morphism. Let $\mathcal{E}$ be an Azumaya algebra over $X$ such that

$$
H^{>0}(X, \mathcal{E})=0 .
$$

Assume that the algebra $\mathbf{A}=\mathcal{E}(X)$ has a finite global dimension. Then the functor

$$
D^{b}(\boldsymbol{C o h}(\mathcal{E})) \rightarrow D^{b}\left(\mathcal{M o d}^{\mathrm{fg}}(\mathbf{A})\right), \quad \mathcal{F} \mapsto \operatorname{RHom}_{\boldsymbol{C o h}(\mathcal{E})}(\mathcal{E}, \mathcal{F})
$$

is an equivalence between the bounded derived category of sheaves of coherent $\mathcal{E}$ modules on $X$ and the bounded derived category of finitely generated $\mathbf{A}$-modules.

To apply this proposition one must prove the following four steps:

- the rational DAHA and its spherical subalgebra are Morita equivalent (under some restrictions on parameters),

- the Chow morphism of the Hilbert scheme $X=\operatorname{Hilb}\left(\mathbb{A}^{2}\right)$ of finite length subschemes of the affine plane is a resolution of singularities of the spectrum of the center of the spherical rational DAHA,

- there is an Azumaya algebra $\mathcal{E}$ over $X$ such that $H^{>0}(X, \mathcal{E})=0$ and $\mathcal{E}(X)$ is isomorphic to the spherical rational DAHA,

- the rational DAHA have a finite global dimension.

In our case we follow these ideas: each of the four steps has an analogue, though all statements need different proofs. Here are a few words concerning the layout of the paper.

In section 1 we first introduce a quantum analogue of DO (differential operators) on $G L_{n} \times \mathbb{P}^{n-1}$. Then we construct a "deformed Harish-Chandra homomorphism" from a ring of QDO (quantum differential operators) to a quantum torus. More precisely, sections 1.3 to 1.5 contain generalities on Heisenberg doubles, quantum moment maps and quantum reduction. In sections 1.6 to 1.10 this is applied to 
the quantum deformation of the enveloping algebra of $\mathfrak{g l}_{n}$. An essential ingredient consists of twisting the product of the Heisenberg double by an explicit cocycle given in terms of the $R$-matrix. This yields a new ring, denoted by $\mathbb{D}^{\prime}$. There are two reasons to do so. The first one is that the quantum adjoint action on the quantized function ring of $G L_{n}$ is not an Hopf algebra action while the adjoint action on $\mathbb{D}^{\prime}$ is a Hopf algebra action. The second one is that the Poisson bracket on the center of the DAHA at roots of unity, given by Hayashi's construction, is the Ruijsenaars-Schneider system by $\mathrm{O}$ and the latter differs from the Poisson bracket of the Heisenberg double. Proposition 1.8.3 and Lemma 4.1.2(b) relate the twisted ring of QDO to the Ruijsenaars-Schneider system. In section 1.11 we give an analogue of the deformed Harish-Chandra homomorphism used in [BFG]. Our construction is based on the work of Etingof and Kirillov in EK].

In section 2 we specialize the previous constructions to the case where the quantum parameters are roots of unity. Sections 2.2 and 2.3 are technical. The reader may skip them, and return for proofs of statements referred to in the subsequent sections. More precisely, section 2.2 is a reminder of more or less standard facts on quantum groups at roots of unity, while section 2.3 deals with invariants and good filtrations. Observe that the most general form of the DAHA is an algebra over the 3 -dimensional ring $\mathbb{Z}\left[q^{ \pm 1}, t^{ \pm 1}\right]$, while quantum groups are traditionally algebras over a $(\leqslant 2)$-dimensional ring. This is a source of technical difficulties. Another source of problems is that we do not know if the algebras of QDO we consider here are all flat. To solve this we must impose that the order of the modular parameter of the DAHA is large enough. In sections 2.4 to 2.7 we specialize the QDO defined in the first part to roots of unity. The deformed Hilbert scheme mentioned above is introduced in section 2.7. Finally, in section 2.8 we specialize the deformed Harish-Chandra homomorphism to roots of unity.

Section 3 is a reminder on double affine Hecke algebras. A convenient reference for this is Cherednik's book [C]. Theorems 3.1.2 and 3.6.9 are new. The first one proves that the DAHA has a finite global dimension. The second one proves that the DAHA is Morita equivalent to its spherical subalgebra. Observe that 3.6.9 uses completely different techniques than in the rest of the paper. It is based on the $K$-theoretic construction in $[\mathrm{V}]$ and $[\mathrm{VV} 3$. For $q=1$ some of the results in this section can be found in $\mathrm{O}$.

Section 4 contains two parts. Section 4.1 is the analogue of the second step above. There we study the deformed Hilbert scheme $T$ introduced in section 2.6. Note that $T$ is the geometric quotient of an open subset of

$$
\left\{(g, h, v, \varphi) \in G L_{n}(\mathbb{C}) \times G L_{n}(\mathbb{C}) \times T^{*} \mathbb{C}^{n} ; g h-\zeta^{2 l} h g+v \otimes \varphi=0\right\},
$$

where $\zeta$ is an invertible parameter. The basic properties of $T$ are more or less standard. Note however that, contrary to the rational case, the scheme $T$ may not be affine for $\zeta \neq 1$. Section 4.2 is the analogue of the third step above. Theorem 4.2.1 is the main result of the paper. In the first part of 4.2.1 we construct a sheaf of Azumaya algebras $\mathfrak{T}$ over $T$. Observe that, contrary to crystalline differential operators in positive characteristic, our ring of QDO on $G L_{n}$ at roots of unity is not an Azumaya algebra but only a maximal order in a central simple algebra. See Corollary 2.2.4 where precise information is given on the Azumaya locus. This information is deduced from the classification of symplectic leaves of the Heisenberg dual in $[\mathrm{AM}$. To prove that $\mathfrak{T}$ is an Azumaya algebra we use once again symplectic 
geometry. Indeed, the key point is that the Poisson structure on $T$ is nondegenerate and that $\mathfrak{T}$ is a Poisson order over $T$. In the second part of 4.2.1, which is analogous to the third step above, we prove first that the deformed Harish-Chandra homomorphism yields an isomorphism from $H^{0}(T, \mathfrak{T})$ to the spherical DAHA and that there is no higher cohomology. The injectivity uses the following basic facts:

- $T$ is a symplectic variety and the function ring of a smooth, affine, connected, symplectic variety is a simple Poisson ring,

- $\mathfrak{T}$ is an Azumaya algebra over $T$ and for any Azumaya algebra A extensions and contractions yield a bijection from two-sided ideals in $\mathbf{A}$ to ideals in the center $Z(\mathbf{A}) \subset \mathbf{A}$.

On the other hand, the surjectivity uses the following basic fact:

- the spherical DAHA, at roots of unity, is a maximal order in a central simple algebra. Further, for any maximal order $\mathbf{A}$, if $\mathbf{B} \subset \operatorname{Frac}(\mathbf{A})$ is a ring containing $\mathbf{A}$ which is finite over $Z(\mathbf{A})$, then $\mathbf{B}=\mathbf{A}$.

To prove the vanishing of higher cohomology we use a deformation to $q=1$ such that the parameter remains a root of unity at each step. The way to do this is to make a deformation to positive characteristic as in [APW]. Then, to lift the vanishing from positive characteristic to characteristic zero we use a Poisson order argument and the following lemma:

- Let $\mathrm{A} \subset \mathbb{C}$ be a DVR with residue field k. Let $f: X \rightarrow Y$ be a proper morphism of flat Poisson A-schemes such that $Y$ is affine, irreducible and $Y \otimes \mathbb{C}$ is symplectic. If $\mathcal{E} \in \operatorname{Coh}\left(\mathcal{O}_{X}\right)$ is an $\mathcal{O}_{X}$-algebra which is a flat A-module and $\mathcal{E} \otimes \mathbb{C}$ is a Poisson order over $Y \otimes \mathbb{C}$, then

$$
H^{>0}(X \otimes \mathrm{k}, \mathcal{E} \otimes \mathrm{k})=0 \Rightarrow H^{>0}(X \otimes \mathbb{C}, \mathcal{E} \otimes \mathbb{C})=0 .
$$

Applications to the representation theory of DAHA at roots of unity are given in Theorem 4.2.7. We do not insist on this, and we will come back to this in a future publication.

Finally, let us make a comment on the structure of the paper. It ends with a large appendix. To facilitate the reading we have put there some standard results for which we did not find an appropriate reference and technical lemmas whose proof was not important for reading the main arguments.

Acknowledgments. We would like to thank H. H. Andersen, A. Braverman, and C. De Concini for valuable discussions.

\section{Quantum GRoups AND QDO}

1.1. Groups and root systems. Let $\mathrm{A}$ be a commutative ring and $n$ an integer $>0$. We denote by $G L_{n, \mathrm{~A}}, S L_{n, \mathrm{~A}}$ the general and the special linear groups over A. If no confusion is possible we omit the ring $\mathrm{A}$ and we abbreviate

$$
G=G_{\mathrm{A}}=G L_{n, \mathrm{~A}}, \quad S L=S L_{\mathrm{A}}=S L_{n, \mathrm{~A}} .
$$

Let $G_{+}$be the semi-group of all $n \times n$-matrices. Let $H \subset H$ be the subgroup of all diagonal matrices, let $U_{-} \subset G$ be the subgroup of all lower unipotent matrices and let $U_{+} \subset G$ be the subgroup of all upper unipotent matrices. Let $e \in G(\mathrm{~A})$ be the unit and let $\mathfrak{g}$ be the Lie algebra of $G$. 
The elements of the weight lattice $X$ of $G$ will be identified with sequences $\lambda=\left(\lambda_{1}, \ldots, \lambda_{n}\right)$ of integers. Let $\varepsilon_{i}=(0, \ldots, 1, \ldots, 0)$ be the $i$ th standard basis element of $X$. For each $h=\operatorname{diag}\left(h_{1}, h_{2}, \ldots, h_{n}\right) \in H(\mathrm{~A})$ we write

$$
h^{\lambda}=h_{1}^{\lambda_{1}} h_{2}^{\lambda_{2}} \cdots h_{n}^{\lambda_{n}} \in \mathrm{A} .
$$

Let $X_{+}=\left\{\lambda_{1} \geqslant \cdots \geqslant \lambda_{n}\right\}$ be the set of dominant weights in $X$. Let $\Pi \subset X$ be the root system of $G$, let $\Pi_{+}=\left\{\varepsilon_{i}-\varepsilon_{j} ; i<j\right\}$ be the set of positive roots and $S=\left\{\alpha_{i}\right\}$ the set of simple roots. As usual we write $\alpha_{i}=\varepsilon_{i}-\varepsilon_{i+1}$ for $i \in I=\{1,2, \ldots, n-1\}$. Let $Y_{+}, Y \subset X$ be the submonoid and the subgroup generated by the set $S$. Set $\rho=\sum_{\alpha \in \Pi_{+}} \alpha / 2$ and $\theta=\varepsilon_{1}-\varepsilon_{n}$. For each $i=1,2, \ldots, n$ we set $\omega_{i}=\sum_{j \leqslant i} \varepsilon_{j}$. Let

$$
X \times X \rightarrow \mathbb{Z}, \quad\left(\lambda, \lambda^{\prime}\right) \mapsto \lambda \cdot \lambda^{\prime}
$$

be the canonical symmetric bilinear form. We write $\lambda \geqslant \lambda^{\prime}$ provided that

$$
\lambda-\lambda^{\prime} \in Y_{+} \text {. }
$$

We identify the Weyl group of $G$ with the symmetric group on $n$-letters $\Sigma_{n}$ in the usual way. Let $\left\{s_{\alpha}\right\},\left\{s_{i}\right\} \subset \Sigma_{n}$ be the sets of reflections and simple reflections. Let

$$
\widehat{\Sigma}_{n}=\Sigma_{n} \ltimes Y, \quad \widetilde{\Sigma}_{n}=\Sigma_{n} \ltimes X,
$$

the affine Weyl group and the extended affine Weyl group. We will abbreviate

$$
\tau_{\lambda}=(1, \lambda), \quad w=(w, 0) .
$$

The unique affine simple reflection in $\widehat{\Sigma}_{n}$ which does not belong to $\Sigma_{n}$ is denoted by $s_{0}=s_{\theta} \tau_{-\theta}$. Recall that the Abelian group

$$
P=X / Y
$$

acts on $\widehat{\Sigma}_{n}$ by automorphisms of the extended Dynkin diagram and that we have

$$
\widetilde{\Sigma}_{n}=\widehat{\Sigma}_{n} \rtimes P \text {. }
$$

The reflection representation of $\Sigma_{n}$ on $X$ yields a representation of $\widetilde{\Sigma}_{n}$ on the $\mathbb{Z}$ module $\widetilde{X}=X \oplus \mathbb{Z} \delta$ such that

$$
\tau_{\lambda}\left(\lambda^{\prime}\right)=\lambda^{\prime}-\left(\lambda \cdot \lambda^{\prime}\right) \delta, \quad \tau_{\lambda}(\delta)=\delta, \quad w(\delta)=\delta .
$$

We will put $\alpha_{0}=\delta-\theta \in \widetilde{X}$.

1.2. Schemes and algebras. A filtration of an object $V$ in an Abelian category will always be an ascending sequence of subobjects $F_{i}(V)$ with $i \in \mathbb{Z}$ such that $V=\bigcup_{i} F_{i}(V)$. The filtration is separated if $\bigcap_{i} F_{i}(V)=0$ and it is positive if $F_{-1}(V)=0$. Let $\operatorname{gr}(V)$ be the associated graded object. If $V$ is a $\mathbb{Z}$-graded object let $V^{m}$ be the homogeneous component of degree $m$. We set also

$$
V^{+}=\bigoplus_{m \geqslant 0} V^{m}, \quad V^{>0}=\bigoplus_{m>0} V^{m}
$$

All rings or algebras are assumed to be unital. We will use the following abbreviations: ID for integral domain, CID for commutative integral domain, CNID for commutative Noetherian integral domain, NID for Noetherian integral domain and DVR for discrete valuation ring. Let $\mathrm{A}$ be a commutative ring. If it is clear from the context, we write $V \otimes W, V^{*}, \operatorname{Hom}(V, W)$ for $V \otimes_{\mathrm{A}} W, \operatorname{Hom}_{\mathrm{A}}(V, \mathrm{~A})$, $\operatorname{Hom}_{\mathrm{A}}(V, W)$ and $(:)$ for the canonical pairing $V^{*} \otimes V \rightarrow \mathrm{A}$. 
Fix any group $G$. A $G$-ring $\mathrm{A}$ is a ring with a $G$-action by ring automorphisms. If $\mathrm{A}$ is commutative, a $G$-equivariant A-algebra is a $G$-ring $\mathbf{A}$ with a morphism of $G$-rings $\mathrm{A} \rightarrow \mathbf{A}$. A $G$-equivariant A-module is an A-module with a compatible $G$-action. Let $\boldsymbol{M o d}(\mathbf{A}, G)$ denote the category of $G$-equivariant $\mathbf{A}$-modules.

If $\mathbf{A}$ is a ring and $i \neq j \in\{1,2,3\}$, let $\mathbf{A}^{\otimes 2} \subset \mathbf{A}^{\otimes 3}, a \mapsto a_{i j}$ be the inclusion obtained by inserting 1 into the component not named. Let $\mathbf{A}_{\circ}$ be the opposite ring and $Z(\mathbf{A})$ the center. If the multiplicative system generated by an element $a \in \mathbf{A}$ is a denominator set, we write $\mathbf{A}_{a}$ for the corresponding quotient ring. Let $\operatorname{Frac}(\mathbf{A})$ denote the quotient ring of $\mathbf{A}$ whenever it is defined. If $\mathbf{A}$ is a $\mathbb{Z}$-graded ring and $a \in Z(\mathbf{A})$ is a homogeneous element, let $\mathbf{A}_{(a)}$ be the degree 0 homogeneous component of the quotient ring $\mathbf{A}_{a}$. We will use the same notation for a $\mathbb{Z}$-graded $\mathbf{A}$-module. If $\mathbf{A}$ is an A-algebra let $\operatorname{Der}_{\mathrm{A}}(\mathbf{A})$ be the set of $\mathrm{A}$-linear derivations of $\mathbf{A}$. If the ring $\mathrm{A}$ is clear from the context, we abbreviate $\operatorname{Der}(\mathbf{A})=\operatorname{Der}_{\mathrm{A}}(\mathbf{A})$. Given an A-linear map $\chi: \mathbf{A} \rightarrow$ A let $\mathbf{A}^{\chi}$ be the kernel of $\chi$. We will write $\operatorname{lgd}(\mathbf{A})$ for the left global dimension of $\mathbf{A}$ and $\operatorname{fd}(V), \operatorname{pd}(V)$ for the flat and projective dimension of a left A-module $V$. If confusion may happen, we write $\operatorname{fd}_{\mathbf{A}}(V), \operatorname{pd}_{\mathbf{A}}(V)$. If $\varphi \in V^{*}$ and $v \in V$, let $c_{\varphi, v}$ be the corresponding matrix coefficient, i.e., the linear form on A such that $a \mapsto \varphi(a v)$.

Let $\boldsymbol{M} \boldsymbol{\operatorname { d o d }}(\mathbf{A})$ be the category of left $\mathbf{A}$-modules and let $\mathcal{M}_{\boldsymbol{o d}}{ }^{\mathrm{lf}}(\mathbf{A})$ be the full subcategory consisting of locally finite modules. Let $\mathcal{M}_{\boldsymbol{o d}}(\mathbf{A}), \boldsymbol{M}_{\boldsymbol{o d}} \boldsymbol{d}_{r}^{\mathrm{lf}}(\mathbf{A})$ be the corresponding categories of right modules. Let

$$
\boldsymbol{M} \operatorname{Mod}(\mathbf{A}) \rightarrow \operatorname{Mod}^{\mathrm{lf}}(\mathbf{A}), V \mapsto V^{\mathrm{lf}}
$$

be the right adjoint to the canonical embedding. Let $\mathcal{G} \boldsymbol{r}(\mathbf{A})$ be the category of $\mathbb{Z}$-graded left $\mathbf{A}$-modules and let $\mathcal{Q} \boldsymbol{g r}(\mathbf{A})$ be the quotient by the full subcategory consisting of torsion modules; see $\mathrm{AZ}$.

Unless specified otherwise a scheme is a Noetherian separated $\mathbb{Z}$-scheme. We will call variety a reduced separated scheme of finite type over an algebraically closed field. For any commutative ring $\mathrm{A}$ and any scheme $X$ let $X(\mathrm{~A})$ be the set of Apoints of $X$. We will write $\mathcal{O}=\mathcal{O}_{X}$ if the scheme $X$ is clear from the context. Let $\mathcal{C o h}(\mathcal{O}), \mathcal{Q} \operatorname{coh}(\mathcal{O})$ be the categories of coherent and quasi-coherent sheaves. Let $K(X)$ be the function field of $X$. An action of an algebraic group $G$ on $X$ will always mean a rational action, i.e., an action obtained from a coaction of the Hopf algebra $\mathcal{O}(G)$.

Given an algebra $\mathcal{E}$ in $\operatorname{Coh}(\mathcal{O})$ let

$$
\mathcal{Q} \operatorname{coh}(\mathcal{E}) \subset \mathcal{Q} \operatorname{coh}(\mathcal{O}), \quad \operatorname{Coh}(\mathcal{E}) \subset \mathcal{C o h}(\mathcal{O})
$$

be the subcategories of sheaves with a structure of left $\mathcal{E}$-module. Morphisms are $\mathcal{E}$-linear homomorphisms. Recall that $\mathcal{E}$ is an Azumaya algebra over $X$ if it is $\mathcal{O}$ coherent and if, for all closed points $x$ of $X$, the stalk $\mathcal{E}_{x}$ is an Azumaya algebra over the local ring $\mathcal{O}_{x}$; see [M2, IV.2].

If $X=\operatorname{Proj}(\mathrm{A})$ and $\mathrm{A}$ is a $\mathbb{Z}_{+}$-graded commutative ring, let

$$
\mathcal{Q g r}(\mathrm{A}) \rightarrow \mathcal{Q} \operatorname{coh}\left(\mathcal{O}_{X}\right), M \mapsto \widetilde{M}
$$

be Serre's localization functor. We will use the same notation for the localization functor on an affine scheme.

Assume that $\mathrm{k}$ is a field of characteristic $p>0$. Set $l=p^{e}$ with $e$ an integer $>0$. Given a k-scheme $X$, let $X^{(e)}$ be its eth Frobenius twist. Recall that $X^{(e)}$ coincides 
with $X$ as a scheme but that it is equipped with a different k-linear structure. The $e$-th power of the Frobenius homomorphism is an affine morphism

$$
\operatorname{Fr}^{e}: X \rightarrow X^{(e)}
$$

which yields a bijection on the sets of k-points. So we may identify the sheaf $\mathcal{O}_{X}$ over $X$ with the sheaf $\left(\mathrm{Fr}^{e}\right)_{*} \mathcal{O}_{X}$ over $X^{(e)}$. If $X$ is a reduced scheme, the $l$ th power map $\mathcal{O}_{X(e)} \rightarrow\left(\mathrm{Fr}^{e}\right)_{*} \mathcal{O}_{X}$ is injective. Under the identification above it yields an isomorphism between the sheaf $\mathcal{O}_{X^{(e)}}$ and the subsheaf

$$
\mathcal{O}_{X}^{l}=\left\{f^{l} ; f \in \mathcal{O}_{X}\right\} \subset \mathcal{O}_{X} .
$$

If $Y \subset X$ is a closed subscheme, then $Y^{(e)}$ is a closed subscheme of $X^{(e)}$. If $Y$ is closed, reduced and $\mathcal{I} \subset \mathcal{O}_{X}$ is its ideal sheaf, then

$$
\mathcal{O}_{Y^{(e)}} \simeq \mathcal{O}_{Y}^{l} \simeq \mathcal{O}_{X}^{l} / \mathcal{I}^{[l]},
$$

where $\mathcal{I}^{[l]} \subset \mathcal{O}_{X}^{l}$ is the ideal generated by the $l$ th powers of elements of $\mathcal{I}$.

1.3. Hopf algebras. Let A be a commutative ring. Let $\mathbf{H}$ be a Hopf algebra. We will always assume that $\mathbf{H}$ is a Hopf algebra over A which is free as an A-module and with a bijective antipode. Let $\varepsilon, m, \Delta, \iota$ be the counit, the multiplication, the coproduct and the antipode of $\mathbf{H}$. Let $\bar{m}, \bar{\Delta}, \bar{\iota}$ be the opposite maps. For each $h \in \mathbf{H}$ we write $\Delta(h)=h_{1} \otimes h_{2}$ and $\Delta^{2}(h)=($ id $\otimes \Delta) \Delta(h)=h_{1} \otimes h_{2} \otimes h_{3}$.

The opposite Hopf algebra, the co-opposite Hopf algebra, the tensor square Hopf algebra and the enveloping Hopf algebra associated with $\mathbf{H}$ are

$$
\mathbf{H}_{\circ}=(\mathbf{H}, \bar{m}, \Delta, \bar{\iota}), \quad \mathbf{H}^{\circ}=(\mathbf{H}, m, \bar{\Delta}, \bar{\iota}), \quad \mathbf{H}^{\otimes 2}=\mathbf{H} \otimes \mathbf{H}, \quad \mathbf{H}^{e}=\mathbf{H}^{\circ} \otimes \mathbf{H},
$$

respectively. The coproduct and the antipode of $\mathbf{H}^{\otimes 2}$ are

$$
\Delta\left(h \otimes h^{\prime}\right)=\left(h_{1} \otimes h_{1}^{\prime}\right) \otimes\left(h_{2} \otimes h_{2}^{\prime}\right), \quad \iota\left(h \otimes h^{\prime}\right)=\iota(h) \otimes \iota\left(h^{\prime}\right) .
$$

Fix a normal left 2-cocycle, i.e., an invertible element $c \in \mathbf{H}^{\otimes 2}$ such that

$$
(\varepsilon \otimes \mathrm{id})(c)=(\mathrm{id} \otimes \varepsilon)(c)=1 \otimes 1, \quad(\Delta \otimes \mathrm{id})(c)(c \otimes 1)=(\mathrm{id} \otimes \Delta)(c)(1 \otimes c) .
$$

A normal left 2-cocycle is called a twist. The twisted Hopf algebra associated with $c$ is

$$
\mathbf{H}_{c}=\left(\mathbf{H}, m, \Delta_{c}, \iota_{c}\right)
$$

with $\Delta_{c}(h)=c^{-1} \Delta(h) c$. We will say that the Hopf algebras $\mathbf{H}, \mathbf{H}_{c}$ are equivalent.

Left or right $\mathbf{H}$-actions are denoted by the symbols $\triangleright$ and $\triangleleft$. We identify $(\mathbf{H}, \mathbf{H})$ bimodules and left $\mathbf{H}^{e}$-modules so that $\left(h \otimes h^{\prime}\right) \triangleright v=h^{\prime} \triangleright v \triangleleft \iota(h)$. We will omit the action symbol when it is clear from the context.

Let $\mathbf{H}^{*}$ be the dual of $\mathbf{H}$, i.e., the set of all linear maps $\mathbf{H} \rightarrow$ A. It is an A-algebra and a $\mathbf{H}^{e}$-module. The restricted dual of $\mathbf{H}$ relative to the tensor category of $\mathbf{H}$ modules which are finite and projective over A, i.e., the sum of all $\mathbf{H}^{e}$-submodules of $\mathbf{H}^{*}$ which are of finite rank as A-modules, is a Hopf algebra.

Let $\mathbf{H}^{\prime} \subset \mathbf{H}$ an A-subalgebra and $\chi: \mathbf{H}^{\prime} \rightarrow \mathrm{A}$ an A-algebra homomorphism. The set of $\left(\mathbf{H}^{\prime}, \chi\right)$-invariants in a $\mathbf{H}^{\prime}$-module $V$ is

$$
V^{\mathbf{H}^{\prime}, \chi}=\left\{v \in V ; h v=\chi(h) v, \forall h \in \mathbf{H}^{\prime}\right\} .
$$

We will abbreviate $V^{\mathbf{H}^{\prime}}=V^{\mathbf{H}^{\prime}, \varepsilon}$ and call it the space of $\mathbf{H}^{\prime}$-invariants in $V$.

We will use the symbol $\mathbf{U}$ for a quasi-triangular Hopf algebra. The $R$-matrix is denoted by $R=\sum_{s} r_{s}^{+} \otimes r_{s}^{-}$. Recall that

$$
R \Delta(u)=\bar{\Delta}(u) R, \quad(\Delta \otimes \mathrm{id})(R)=R_{13} R_{23}, \quad(\mathrm{id} \otimes \Delta)(R)=R_{13} R_{12},
$$




$$
(\varepsilon \otimes \mathrm{id})(R)=(\mathrm{id} \otimes \varepsilon)(R)=1, \quad(\iota \otimes \iota)(R)=R, \quad(\iota \otimes \mathrm{id})(R)=R^{-1} .
$$

Let $\mathbf{F}$ be the restricted dual of $\mathbf{U}$ relative to the tensor category of $\mathbf{U}$-modules which are finite and projective over A. There exist Hopf algebra homomorphisms

$$
R^{+}: \mathbf{F}^{\circ} \rightarrow \mathbf{U}, f \mapsto(f \otimes \text { id }: R), \quad R^{-}: \mathbf{F}^{\circ} \rightarrow \mathbf{U}, f \mapsto\left(f \otimes \text { id }: R_{21}^{-1}\right) .
$$

Before we go on let us give a few examples.

\subsubsection{Examples.}

(a) The element $R_{23} \in \mathbf{U}^{\otimes 4}$ is a twist of the Hopf algebra $\mathbf{U}^{\otimes 2}$. Let $\mathbf{U}^{[2]}$ be the corresponding twisted Hopf algebra. It is quasi-triangular with $\Delta_{c}(u)=$ $R_{23}^{-1} \Delta(u) R_{23}$ and $\iota_{c}(u)=R_{21} \iota(u) R_{21}^{-1}$. A computation yields

$$
\Delta_{c}\left(u \otimes u^{\prime}\right)=\sum_{s, t} u_{1} \otimes \iota\left(r_{s}^{+}\right) u_{1}^{\prime} r_{t}^{+} \otimes r_{s}^{-} u_{2} r_{t}^{-} \otimes u_{2}^{\prime}
$$

Let $\bar{\Delta}$ denote the coproduct of $\mathbf{F}^{\circ}$. By [M1, 7.28, 7.31] there is a Hopf algebra homomorphism

$$
\Lambda=\left(R^{+} \otimes R^{-}\right) \circ \bar{\Delta}: \mathbf{F}^{\circ} \rightarrow \mathbf{U}^{[2]} .
$$

(b) The element $R_{35} R_{34}$ is a twist of the Hopf algebra $\mathbf{U}^{[2]} \otimes \mathbf{U}$. Let $\mathbf{U}^{[3]}$ be the corresponding twisted Hopf algebra. The maps $\Delta, \Delta^{2}$ are Hopf algebra inclusions of $\mathbf{U} \subset \mathbf{U}^{[2]}, \mathbf{U}^{[3]}$. A direct computation yields the following formula in $\mathbf{U}^{[3]}$ :

$$
\begin{aligned}
\Delta\left(v \otimes u^{\prime} \otimes 1\right)= & \sum_{s, t, x, y, z} v_{1} \otimes \iota\left(r_{s}^{+}\right) u_{1}^{\prime} r_{t}^{+} \\
& \otimes \iota\left(r_{z}^{+} r_{y}^{+} r_{x}^{+}\right) \otimes r_{x}^{-} r_{s}^{-} v_{2} r_{t}^{-} \iota\left(r_{z}^{-}\right) \otimes\left(\mathrm{ad} r_{y}^{-}\right)\left(u_{2}^{\prime}\right) \otimes 1 .
\end{aligned}
$$

(c) The Hopf algebra $\mathbf{U}^{e}$ is equivalent to $\mathbf{U}^{[2]}$, the corresponding twist is $R_{13} R_{23}$. The Hopf algebra $\left(\mathbf{U}^{e}\right)^{\otimes 2}$ is equivalent to $\mathbf{U}^{\circ} \otimes \mathbf{U}^{[3]}$, the corresponding twist is $R_{37} R_{36} R_{47} R_{46}$.

Given a representation $V$ of $\mathbf{H}^{e}$ and an element $h \in \mathbf{H}$ we will set $(\operatorname{ad} h) \triangleright v=$ $\Delta(h) \triangleright v$. If $\mathbf{H}$ is quasi-triangular and $V$ is a representation $\mathbf{H}^{[2]}$ we will also set $(\operatorname{ad} h) \triangleright v=\Delta(h) \triangleright v$. In both cases this yields a representation of $\mathbf{H}$ on $V$. We call it the adjoint $\mathbf{H}$-action.

The adjoint $\mathbf{H}$-action on $\mathbf{H}$ is given by $(\operatorname{ad} h)\left(h^{\prime}\right)=h_{1} h^{\prime} \iota\left(h_{2}\right)$. We may also use the right adjoint action, which is defined by $\left(\operatorname{ad}_{r} h\right)\left(h^{\prime}\right)=\iota\left(h_{1}\right) h^{\prime} h_{2}$. Let $\mathbf{H}^{\mathrm{lf}} \subset \mathbf{H}$ denote the locally finite part of the adjoint action. A subalgebra $\mathbf{H}^{\prime} \subset \mathbf{H}$ is said to be normal if it is preserved by the adjoint $\mathbf{H}$-action.

The following properties are standard. See section A.1 for details.

\subsubsection{Examples.}

(a) $\mathbf{U} \otimes 1, \mathbf{U}^{[2]} \otimes 1$ are normal left coideal subalgebras of $\mathbf{U}^{[2]}, \mathbf{U}^{[3]}$, respectively.

(b) The map $\kappa: \mathbf{F} \rightarrow \mathbf{U}^{\text {lf }}, f \mapsto R^{-} \iota\left(f_{1}\right) R^{+}\left(f_{2}\right)$ is (adU)-linear. It is injective if $\mathbf{U}$ is factorizable. Taking $R_{21}^{-1}$ as the $R$-matrix instead of $R$, we get another (adU)-linear map $\bar{\kappa}$ such that $\bar{\kappa}(f)=R^{+} \iota\left(f_{1}\right) R^{-}\left(f_{2}\right)$. We have $m(\bar{\kappa} \otimes \kappa) \Delta=m(\kappa \otimes \bar{\kappa}) \Delta=\varepsilon$.

(c) There are linear isomorphisms $\varpi_{i}: \mathbf{U}^{\otimes i} \rightarrow \mathbf{U}^{[i]}, i=2,3$, which commute with the adjoint actions and are such that $\Delta \kappa=\varpi_{2}(\kappa \otimes \kappa) \Delta$ and $\Delta^{2} \kappa=$ $\varpi_{3}(\kappa \otimes \kappa \otimes \kappa) \Delta^{2}$. 
1.4. Smash products. Let $A$ be a commutative ring and $\mathbf{H}$ a Hopf A-algebra. Let $\mathbf{A}$ be an A-algebra. An $\mathbf{H}$-action on $\mathbf{A}$ is a representation such that

$$
h \triangleright 1=\varepsilon(h) 1, \quad h \triangleright a a^{\prime}=\left(h_{1} \triangleright a\right)\left(h_{2} \triangleright a^{\prime}\right), \quad \forall h, a, a^{\prime} .
$$

If the action is clear from the context we simply say that $\mathbf{A}$ is an $\mathbf{H}$-algebra. The following properties are immediate: the locally finite part $\mathbf{A}^{\mathrm{lf}}$ of the $\mathbf{H}$-action on $\mathbf{A}$ is an A-subalgebra and the coaction map is an algebra homomorphism $\mathbf{A} \rightarrow \mathbf{H}^{*} \otimes \mathbf{A}$.

For any left coideal subalgebra $\mathbf{H}^{\prime} \subset \mathbf{H}$ let $\boldsymbol{M} \boldsymbol{o d}\left(\mathbf{A}, \mathbf{H}^{\prime}\right)$ be the category of the $\mathbf{H}^{\prime}$-equivariant A-modules, i.e., the category of the $\mathbf{A}$-modules $V$ with an $\mathbf{H}^{\prime}$-action such that

$$
h \triangleright(a v)=\left(h_{1} \triangleright a\right)\left(h_{2} \triangleright v\right), \quad \forall h, a, v .
$$

Let $\mathbf{A}$ be an $\mathbf{H}$-algebra. Fix a twist $c$ of $\mathbf{H}$. We define an $\mathbf{H}_{c}$-algebra $\mathbf{A}_{c}$ by defining a new multiplication on $\mathbf{A}$ such that

$$
m_{c}\left(a \otimes a^{\prime}\right)=m\left(c \triangleright\left(a \otimes a^{\prime}\right)\right), \quad \forall a, a^{\prime} .
$$

The $\mathbf{H}_{c}$-action on $\mathbf{A}_{c}$ is the same as the $\mathbf{H}$-action on $\mathbf{A}$. We say that the $\mathbf{H}$-algebra $\mathbf{A}$ is equivalent to the $\mathbf{H}_{c}$-algebra $\mathbf{A}_{c}$.

Given $V \in \operatorname{Mod}(\mathbf{A}, \mathbf{H})$ we define a new object $V_{c} \in \operatorname{Mod}\left(\mathbf{A}_{c}, \mathbf{H}_{c}\right)$ as follows. Let $\alpha: \mathbf{A} \otimes V \rightarrow V$ denote the $\mathbf{A}$-action on $V$. There is an $\mathbf{A}_{c}$-action on $V$ given by $a \otimes v \mapsto \alpha(c \triangleright(a \otimes v))$. Together with the original $\mathbf{H}$-action on $V$ this yields $V_{c}$.

Before we go on let us give a few examples. See section A.2 for details.

\subsubsection{Examples.}

(a) It is known that $\mathbf{F}$ is a $\mathbf{U}^{e}$-algebra such that $\left(u \otimes u^{\prime}\right) \triangleright f=u^{\prime} \triangleright f \triangleleft \iota(u)$, where $u \triangleright f=\left(f_{2}: u\right) f_{1}$ and $f \triangleleft u=\left(f_{1}: u\right) f_{2}$. The corresponding adjoint action is given by $(\operatorname{ad} u) f=u_{2} \triangleright f \triangleleft \iota\left(u_{1}\right)$. We may also use the right adjoint action which is given by $\left(\operatorname{ad}_{r} u\right) f=\iota\left(u_{2}\right) \triangleright f \triangleleft u_{1}$.

The adjoint $\mathbf{U}$-action on $\mathbf{U}$ is a Hopf algebra action, the adjoint $\mathbf{U}$-action on $\mathbf{F}$ is not. More generally, the adjoint $\mathbf{U}$-action on a $\mathbf{U}^{[2]}$-algebra is a Hopf algebra action, while the adjoint $\mathbf{U}$-action on a $\mathbf{U}^{e}$-algebra is not.

The multiplication $m$ in $\mathbf{F}$ satisfies the following relation:

$$
\sum_{s} m\left(\left(f \triangleleft r_{s}^{+}\right) \otimes\left(f^{\prime} \triangleleft r_{s}^{-}\right)\right)=\sum_{s} m\left(\left(r_{s}^{-} \triangleright f^{\prime}\right) \otimes\left(r_{s}^{+} \triangleright f\right)\right) .
$$

(b) Let $\mathbf{F}^{\prime}$ be the $\mathbf{U}^{[2]}$-algebra equivalent to the $\mathbf{U}^{e}$-algebra $\mathbf{F}$. The $\mathbf{U}^{[2]}$-action on $\mathbf{F}^{\prime}$ is given by $\left(u \otimes u^{\prime}\right) \triangleright f=u^{\prime} \triangleright f \triangleleft \iota(u)$. Let $m, m^{\prime}$ be the multiplication in $\mathbf{F}, \mathbf{F}^{\prime}$, respectively. We have

$$
\begin{aligned}
m\left(f \otimes f^{\prime}\right) & =\sum_{s} m^{\prime}\left(\left(\operatorname{ad} r_{s}^{+}\right)(f) \otimes\left(f^{\prime} \triangleleft r_{s}^{-}\right)\right), \\
m^{\prime}\left(f \otimes f^{\prime}\right) & =\sum_{s} m\left(\left(\operatorname{ad} r_{s}^{+}\right)(f) \otimes\left(f^{\prime} \triangleleft \iota\left(r_{s}^{-}\right)\right)\right) .
\end{aligned}
$$

In particular, the following relation holds:

$$
\sum_{s, t} m^{\prime}\left(\left(r_{s}^{+} \triangleright f \triangleleft r_{t}^{-}\right) \otimes\left(f^{\prime} \triangleleft r_{t}^{+} r_{s}^{-}\right)\right)=\sum_{s, t} m^{\prime}\left(\left(r_{t}^{+} r_{s}^{-} \triangleright f^{\prime}\right) \otimes\left(r_{s}^{+} \triangleright f \triangleleft r_{t}^{-}\right)\right) .
$$

The maps $\kappa, \bar{\kappa}$ are (adU)-algebra homomorphisms $\mathbf{F}^{\prime} \rightarrow \mathbf{U}^{\text {lf }}$. Composing the counit of $\mathbf{U}$ and the map $\kappa$ we get a homomorphism $\mathbf{F}^{\prime} \rightarrow$ A. This map is equal to the counit of $\mathbf{F}$. 
(c) Fix a $\mathbf{U}$-algebra $\mathbf{A}$. Twisting the multiplication in $\mathbf{A}^{\otimes 2}$ by the twist $R_{23}$ yields a $\mathbf{U}^{[2]}$-algebra $\mathbf{A}^{(2)}$. Now set $\mathbf{A}=\mathbf{U}$ or $\mathbf{F}^{\prime}$ with the adjoint $\mathbf{U}$ action. We get the $\left(\operatorname{ad} \mathbf{U}^{[2]}\right)$-algebras $\mathbf{U}^{(2)},\left(\mathbf{F}^{\prime}\right)^{(2)}$, respectively. The map $\varpi_{2}$ in 1.3.3(c) is an (ad $\left.\mathbf{U}^{[2]}\right)$-algebra homomorphism $\mathbf{U}^{(2)} \rightarrow \mathbf{U}^{[2]}$.

(d) Given an $\mathbf{H}$-algebra $\mathbf{A}$ and a left or right coideal subalgebra $\mathbf{H}^{\prime} \subset \mathbf{H}$ the set of $\mathbf{H}^{\prime}$-invariants $\mathbf{A}^{\mathbf{H}^{\prime}}$ is an A-subalgebra of $\mathbf{A}$.

Recall that $\mathbf{A}$ is an $\mathbf{H}$-algebra. The smash product of $\mathbf{A}$ and $\mathbf{H}$ is the A-algebra $\mathbf{A} \sharp \mathbf{H}$ generated by $\mathbf{A}, \mathbf{H}$ with the additional relations $h a=\left(h_{1} \triangleright a\right) h_{2}$ for all $h, a$. It is an $\mathbf{H}$-overalgebra of $\mathbf{A}$ and of $\mathbf{H}$, for the $\mathbf{H}$-action given by

$$
h^{\prime} \triangleright(a h)=\left(h_{1}^{\prime} \triangleright a\right)\left(\operatorname{ad} h_{2}^{\prime}\right)(h) .
$$

Let $\ell, \partial_{\triangleright}$ be the obvious inclusions $\mathbf{A}, \mathbf{H} \subset \mathbf{A} \sharp \mathbf{H}$. An $\mathbf{A} \sharp \mathbf{H}$-module is the same as an $\mathbf{H}$-equivariant $\mathbf{A}$-module. We define the basic representation of $\mathbf{A} \sharp \mathbf{H}$ to be $\mathbf{A}$, with the left multiplication by $\mathbf{A}$ and the natural action of $\mathbf{H}$.

Given a left coideal subalgebra $\mathbf{H}^{\prime} \subset \mathbf{H}$ let $\mathbf{A} \sharp \mathbf{H}^{\prime} \subset \mathbf{A} \sharp \mathbf{H}$ be the subalgebra generated by $\ell(\mathbf{A}), \partial_{\triangleright}\left(\mathbf{H}^{\prime}\right)$. It is preserved by the $\mathbf{H}$-action if $\mathbf{H}^{\prime}$ is a normal subalgebra. The restriction of the basic representation of $\mathbf{A} \sharp \mathbf{H}$ to $\mathbf{A} \sharp \mathbf{H}^{\prime}$ is called again the basic representation of $\mathbf{A} \sharp \mathbf{H}^{\prime}$. The following is proved in section A.2.

1.4.2. Proposition. For each twist $c$ there is an isomorphism of A-algebras $\Xi$ : $\mathbf{A}_{c} \sharp \mathbf{H}_{c} \rightarrow \mathbf{A} \sharp \mathbf{H}$ which factors through the identity of the basic representations $\mathbf{A}_{c} \rightarrow \mathbf{A}$.

\subsubsection{Examples.}

(a) The obvious inclusions $\mathbf{U}^{\circ}, \mathbf{U} \subset \mathbf{U}^{e}$ yield normal left coideal subalgebras of $\mathbf{U}^{e}$. Thus $\mathbf{F} \sharp \mathbf{U}^{\circ}, \mathbf{F} \sharp \mathbf{U}$ are $\mathbf{U}^{e}$-subalgebras of the smash-product $\mathbf{F} \sharp \mathbf{U}^{e}$.

(b) The inclusion $\mathbf{U} \subset \mathbf{U}^{[2]}$ maps $\mathbf{U}$ onto a normal left coideal subalgebra of $\mathbf{U}^{[2]}$; see 1.3.3(a). Further, $\mathbf{F}^{\prime}$ is a $\mathbf{U}^{[2]}$-algebra by 1.4.1(b). Thus $\mathbf{F}^{\prime} \sharp \mathbf{U}$, $\mathbf{F}^{\prime} \sharp \mathbf{U}^{[2]}$ are both $\mathbf{U}^{[2]}$-algebras. The isomorphism $\Xi: \mathbf{F}^{\prime} \sharp \mathbf{U}^{[2]} \rightarrow \mathbf{F} \sharp \mathbf{U}^{e}$ in 1.4.2 factors to an A-algebra isomorphism $\mathbf{F}^{\prime} \sharp \mathbf{U} \rightarrow \mathbf{F} \sharp \mathbf{U}^{\circ}$.

(c) By definition of $\mathbf{F}$ we have $\mathbf{F} \subset \mathbf{U}^{*}$. We also have $\mathbf{U} \subset \mathbf{F}^{*}$ iff the natural pairing $(:): \mathbf{F} \times \mathbf{U} \rightarrow \mathrm{A}$ is nondegenerate. If $\mathbf{U} \subset \mathbf{F}^{*}$, then the basic representation of $\mathbf{F} \sharp \mathbf{U}$ on $\mathbf{F}$ is faithful. See section A.2 for details.

1.5. Quantum reduction. Let $\mathrm{A}$ be a commutative ring. Let $\mathbf{H}$ be a Hopf algebra and let $\mathbf{A}$ be an $\mathbf{H}$-algebra. Let $\mathbf{H}^{\prime} \subset \mathbf{H}$ be a left coideal subalgebra.

A quantum moment map for $\mathbf{A}$ (QMM for short) is an A-algebra homomorphism $\partial_{\triangleright}: \mathbf{H}^{\prime} \rightarrow \mathbf{A}$ such that

$$
\left(h_{1} \triangleright a\right) \partial_{\triangleright}\left(h_{2}\right)=\partial_{\triangleright}(h) a, \quad \forall a, h .
$$

Fix the map $\partial_{\triangleright}$. Then the $\mathbf{H}$-action on $\mathbf{A}$ is given by

$$
h^{\prime} \triangleright \ell(a) \partial_{\triangleright}(h)=\ell\left(h_{1}^{\prime} \triangleright a\right) \partial_{\triangleright}\left(\operatorname{ad} h_{2}^{\prime}\right)(h) .
$$

Note that any $V \in \boldsymbol{M} \boldsymbol{o d}(\mathbf{A})$ has a natural structure of an $\mathbf{H}^{\prime}$-equivariant $\mathbf{A}$-module: the $\mathbf{H}^{\prime}$-action is the composition of $\partial_{\triangleright}$ and the $\mathbf{A}$-action on $V$. So we have defined a functor $\operatorname{Mod}(\mathbf{A}) \rightarrow \operatorname{Mod}\left(\mathbf{A}, \mathbf{H}^{\prime}\right)$.

An A-algebra homomorphism $\chi: \mathbf{H}^{\prime} \rightarrow$ A yields an algebra homomorphism

$$
\mathbf{H}^{\prime} \rightarrow \mathbf{H}, \quad h \mapsto h^{\chi}=h_{1} \chi\left(h_{2}\right) .
$$


We will say that $\mathbf{H}^{\prime}$ is $\chi$-stable if it is preserved by this map. Note that we have

$$
\Delta\left(h^{\chi}\right)=h_{1} \otimes h_{2}^{\chi}, \quad\left(\operatorname{ad} h^{\prime}\right)\left(h^{\chi}\right)=\left(\left(\operatorname{ad} h^{\prime}\right) h\right)^{\chi}, \quad \forall h \in \mathbf{H}^{\prime}, h^{\prime} \in \mathbf{H} .
$$

Thus, if $\mathbf{H}^{\prime} \subset \mathbf{H}$ is normal, then

$$
\left(\mathbf{H}^{\prime}\right)^{\chi}=\left\{h^{\chi} ; h \in \mathbf{H}^{\prime}\right\}
$$

is also normal in $\mathbf{H}$. If $\mathbf{H}^{\prime} \subset \mathbf{H}$ is $\chi$-stable and normal, the left ideal

$$
\mathbf{A} \partial_{\triangleright}\left(\mathbf{H}^{\prime}\right)^{\chi} \subset \mathbf{A}
$$

is preserved by the $\mathbf{H}^{\prime}$-action. Thus we may set

$$
\mathbf{A} / / \chi \mathbf{H}^{\prime}=\left(\mathbf{A} / \mathbf{A} \partial_{\triangleright}\left(\mathbf{H}^{\prime}\right)^{\chi}\right)^{\mathbf{H}^{\prime}} .
$$

If $\chi=\varepsilon$ we will abbreviate $\mathbf{A} / / \mathbf{H}^{\prime}=\mathbf{A} / / \varepsilon \mathbf{H}^{\prime}$. The following is proved in section A.3.

1.5.2. Proposition. Let $\mathbf{H}^{\prime} \subset \mathbf{H}$ be a normal $\chi$-stable left coideal subalgebra and let $\partial_{\triangleright}: \mathbf{H}^{\prime} \rightarrow \mathbf{A}$ be a $Q M M$. The following hold:

(a) $\mathbf{A} / / \chi \mathbf{H}^{\prime}$ is an A-algebra,

(b) if $\mathbf{A}$ is a field and $\mathbf{A}$ is a matrix algebra, then $\mathbf{A} / / \chi \mathbf{H}^{\prime}$ is again a matrix algebra,

(c) taking invariants yield a functor $\mathcal{M o d}(\mathbf{A}) \rightarrow \operatorname{Mod}\left(\mathbf{A} / / \chi \mathbf{H}^{\prime}\right), V \mapsto V^{\mathbf{H}^{\prime}, \chi}$.

\subsubsection{Remarks.}

(a) Assume that $V \in \boldsymbol{M} \boldsymbol{M o d}(\mathbf{H})$ is projective of finite rank over A. Then $\operatorname{End}(V)$ is an $\mathbf{H}$-algebra for the $\mathbf{H}$-action such that $\operatorname{End}(V)=V \otimes V^{\circ}$ as a $\mathbf{H}$ module. Here $V^{\circ}$ is the contragredient dual left $\mathbf{H}$-module, i.e., the dual A-module with the action twisted by the antipode. If $\mathbf{A} \subset \operatorname{End}(V)$ is an $\mathbf{H}$-subalgebra, then an algebra homomorphism $\partial_{\triangleright}: \mathbf{H}^{\prime} \rightarrow \mathbf{A}$ is a QMM if $\partial_{\triangleright}(h)(v)=h \triangleright v$ for all $h \in \mathbf{H}^{\prime}, v \in V$.

(b) The inclusion $\partial_{\triangleright}: \mathbf{H} \rightarrow \mathbf{A} \sharp \mathbf{H}$ is a QMM.

(c) Let $\mathbf{H}^{\prime} \subset \mathbf{H}$ be a normal left coideal subalgebra. Let $\chi: \mathbf{H}^{\prime} \rightarrow \mathrm{A}$ be an A-algebra homomorphism. Assume that $\mathbf{H}^{\prime}$ is $\chi$-stable. The assignment $\ell(a) \partial_{\triangleright}(h) \mapsto \ell(a) \partial_{\triangleright}\left(h^{\chi}\right)$ gives an $\mathbf{H}$-algebra endomorphism of $\mathbf{A} \sharp \mathbf{H}^{\prime}$.

(d) Let $\mathbf{H}^{\prime} \subset \mathbf{H}$ be a normal left coideal subalgebra. Let $\chi: \mathbf{H}^{\prime} \rightarrow \mathrm{A}$ be an A-algebra homomorphism. Assume that $\mathbf{H}^{\prime}$ is $\chi$-stable. Let $\mathbf{A}$ be an $\mathbf{H}$-algebra with a QMM $\partial_{\triangleright}: \mathbf{H}^{\prime} \rightarrow \mathbf{A}$. Assume that $\phi: \mathbf{A} \rightarrow \mathbf{A}$ is an $\mathbf{H}$-algebra homomorphism such that $\phi \partial_{\triangleright}(h)=\partial_{\triangleright}\left(h^{\chi}\right)$ for all $h \in \mathbf{H}^{\prime}$. Then $\phi$ factors to an A-algebra isomorphism $\mathbf{A} / / \mathbf{H}^{\prime}=\mathbf{A} / / \chi \mathbf{H}^{\prime}$.

1.6. Quantized enveloping algebra. Fix an indeterminate $q$ and a field extension $\mathbb{Q}(q) \subset \mathcal{K}$. Let $\mathbb{U}$ be the $\mathcal{K}$-algebra generated by $e_{i}, f_{i}, k_{\lambda}, i \in I, \lambda \in X$ with the defining relations

$$
\begin{gathered}
k_{\lambda} e_{i}=q^{\lambda \cdot \alpha_{i}} e_{i} k_{\lambda}, \quad k_{\lambda} f_{i}=q^{-\lambda \cdot \alpha_{i}} f_{i} k_{\lambda}, \quad k_{\lambda} k_{\lambda^{\prime}}=k_{\lambda+\lambda^{\prime}}, \\
{\left[e_{i}, f_{j}\right]=\delta_{i j}\left(k_{\alpha_{i}}-k_{-\alpha_{i}}\right) /\left(q-q^{-1}\right)}
\end{gathered}
$$

and the quantum Serre relations. Here we set $\delta_{i j}=1$ if $i=j$ and zero else. The $\mathcal{K}$-algebra $\mathbb{U}$ is an ID; see [J2, sec. 7.3.4]. To simplify we may write $k_{i}=k_{\alpha_{i}}$. The coproduct and antipode are

$$
\begin{gathered}
\Delta\left(e_{i}\right)=k_{i}^{-1} \otimes e_{i}+e_{i} \otimes 1, \quad \Delta\left(f_{i}\right)=1 \otimes f_{i}+f_{i} \otimes k_{i}, \quad \Delta\left(k_{\lambda}\right)=k_{\lambda} \otimes k_{\lambda}, \\
\iota\left(e_{i}\right)=-k_{i} e_{i}, \quad \iota\left(f_{i}\right)=-f_{i} k_{i}^{-1}, \quad \iota\left(k_{\lambda}\right)=k_{\lambda}^{-1} .
\end{gathered}
$$


Let $\mathbb{U}_{+}, \mathbb{U}_{-}, \mathbb{U}_{0} \subset \mathbb{U}$ be the positive, negative and Cartan part. We fix once and for all a reduced expression of $w_{0}$, the longest element in the Weyl group. Let $e_{\alpha}, f_{\alpha}$, $\alpha \in \Pi_{+}$be the corresponding root vectors in $\mathbb{U}$. We may write $e_{i j}=e_{\alpha}, f_{i j}=f_{\alpha}$ if $\alpha=\varepsilon_{i}-\varepsilon_{j}$. Put

$$
\dot{e}_{\alpha}=\left(q-q^{-1}\right) e_{\alpha}, \quad \dot{f}_{\alpha}=\left(q-q^{-1}\right) f_{\alpha}, \quad \dot{e}_{i}=\dot{e}_{\alpha_{i}}, \quad \dot{f}_{i}=\dot{f}_{\alpha_{i}} .
$$

Consider the Hopf algebra isomorphism $\mathfrak{a}^{\prime}: \mathbb{U} \rightarrow \mathbb{U}_{\circ}$ given by

$$
\mathfrak{a}^{\prime}\left(e_{i}\right)=f_{i} k_{i}^{-1}, \quad \mathfrak{a}^{\prime}\left(f_{i}\right)=k_{i} e_{i}, \quad \mathfrak{a}^{\prime}\left(k_{\lambda}\right)=k_{\lambda} .
$$

The map $\mathfrak{a}=\bar{\iota} \circ \mathfrak{a}^{\prime}=\mathfrak{a}^{\prime} \circ \iota$ is the Cartan involution of $\mathbb{U}$.

Let $X^{\prime}$ be the set of characters $\mathbb{U}_{0} \rightarrow \mathcal{K}$. Given an $\mathbb{U}$-module $V$ and a character $\nu \in X^{\prime}$ let $V_{\nu} \subset V$ be the corresponding weight subspace. We say that $V$ is a weight module if $V=\bigoplus_{\nu} V_{\nu}$ and $\operatorname{dim}\left(V_{\nu}\right)<\infty$ for all $\nu$. Let $V^{*}=\bigoplus_{\nu} V_{\nu}^{*}$ be the dual right weight module. The contragredient dual left weight module $V^{\circ}$ is obtained by twisting the right action on $V^{*}$ by the antipode. For each $\lambda \in X$, the weight lattice of $G$, we have the character $q^{\lambda}: k_{\lambda^{\prime}} \mapsto q^{\lambda \cdot \lambda^{\prime}}$. This yields an inclusion $X \subset X^{\prime}$. We say that the weight module $V$ is of type 1 if all of its weights belong to $X$.

Let $\mathcal{O}(\mathbb{U}) \subset \boldsymbol{M o d}(\mathbb{U})$ be the BGG subcategory. For each $\nu \in X^{\prime}$ let $M(\nu)$ be the Verma module with the highest weight $\nu$ and let $V(\nu)$ be its top. We abbreviate

$$
M(\lambda)=M\left(q^{\lambda}\right), \quad V(\lambda)=V\left(q^{\lambda}\right) .
$$

We will fix once and for all a basis $\left(v_{i}^{\lambda}\right)$ of $V(\lambda)$ for each $\lambda \in X$ which consists of weight vectors such that $v_{1}^{\lambda}$ is a highest weight vector and $v_{1}^{\lambda} \otimes v_{1}^{\lambda^{\prime}}=v_{1}^{\lambda+\lambda^{\prime}}$. Let $\left(\varphi_{i}^{\lambda}\right)$ be the dual basis. We will abbreviate $v_{\lambda}=v_{1}^{\lambda}$ and $\varphi_{\lambda}=\varphi_{1}^{\lambda}$.

1.7. Quantized function algebra. Let $\mathbb{F}$ be the restricted dual of the Hopf algebra $\mathbb{U}$ with respect to the category of finite dimensional modules of type 1 . It is a $\mathbb{U}^{e}$-algebra and an ID; see [J2, sec. 9.1.9]. For each $\lambda \in X_{+}, i, j=1,2, \ldots, n$ we put $c_{\lambda}=c_{\varphi_{\lambda}, v_{\lambda}}$ and $c_{i j}=c_{\varphi_{i}^{\omega_{1}}, v_{j}^{\omega_{1}}}$. Let $\operatorname{cf}(V(\lambda)) \subset \mathbb{F}$ be the vector subspace spanned by the matrix coefficients of $V(\lambda)$. Let $\mathbb{F}_{+} \subset \mathbb{F}$ be the subalgebra generated by the set $\left\{c_{i j}\right\}$.

Let $\mathbb{F}_{0}$ be the $\mathcal{K}$-span of $\left\{q^{\lambda} ; \lambda \in X\right\}$ and let

$$
\varrho_{0}: \mathbb{F} \rightarrow \mathbb{F}_{0}
$$

be the restriction of functions to $\mathbb{U}_{0}$. The map $\varrho_{0}$ is a Hopf algebra homomorphism.

Let $\mathbb{F}^{\prime}$ be as in 1.4.1. Let $\mathbb{F}_{+}^{\prime} \subset \mathbb{F}^{\prime}$ be the subalgebra generated by the set $\left\{c_{i j}\right\}$. The identity $\mathbb{F} \rightarrow \mathbb{F}^{\prime}$ maps $\mathbb{F}_{+}$onto $\mathbb{F}_{+}^{\prime}$.

We need the following quotient rings of $\mathbb{F}$ :

- $\mathbb{F}_{i}$ is the localization at the denominator set generated by $c_{1 i}$ for $i=$

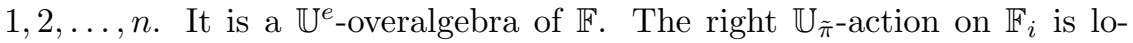
cally finite.

- $\mathbb{F}_{*}$ is the localization at the denominator set generated by $\left\{c_{1 i}\right\}$.

- $\mathbb{F}_{\Sigma}$ is the localization at $\Sigma=\left\{c_{\lambda} ; \lambda \in X_{+}\right\}$; see [J2, 9.1.10(iii)].

Let $\mathbb{U}^{e}, \mathbb{U}^{[2]}, \mathbb{U}^{[3]}$ be as in section 1.3. Notice that the coproduct of $\mathbb{U}^{[2]}, \mathbb{U}^{[3]}$ maps only to a completion of the tensor square. This will have no consequence in the rest of the paper. So we omit it and call $\mathbb{U}^{[2]}, \mathbb{U}^{[3]}$ a Hopf algebra again. Set

$$
\mathbb{U}^{\prime}=\bigoplus_{\lambda \in 2 X_{+}}(\operatorname{ad} \mathbb{U})\left(k_{\lambda}\right), \quad\left(\mathbb{U}^{\prime}\right)^{[2]}=\varpi_{2}\left(\mathbb{U}^{\prime} \otimes \mathbb{U}^{\prime}\right), \quad\left(\mathbb{U}^{\prime}\right)^{[3]}=\varpi_{3}\left(\mathbb{U}^{\prime} \otimes \mathbb{U}^{\prime} \otimes \mathbb{U}^{\prime}\right)
$$


The maps $\varpi_{2}, \varpi_{3}$ are as in 1.3.3(c) and section A.1.

The universal $R$-matrix yields the following endomorphism of $V\left(\omega_{1}\right) \otimes V\left(\omega_{1}\right)$ :

$$
R^{q}=\sum_{i, j} q^{\delta_{i j}} e_{i i} \otimes e_{j j}+\left(q-q^{-1}\right) \sum_{i<j} e_{i j} \otimes e_{j i} .
$$

1.7.1. Proposition. (a) The maps $\kappa, \bar{\kappa}$ yield $(\operatorname{ad} \mathbb{U})$-algebra isomorphisms $\mathbb{F}^{\prime} \rightarrow \mathbb{U}^{\prime}$ such that $\kappa\left(c_{\lambda}\right)=\bar{\kappa} \iota\left(c_{\lambda}\right)=k_{2 \lambda}$. There are the inclusions

$$
\Delta\left(\mathbb{U}^{\prime}\right) \subset\left(\mathbb{U}^{\prime}\right)^{[2]} \subset \mathbb{U} \otimes \mathbb{U}^{\prime}, \quad \Delta^{2}\left(\mathbb{U}^{\prime}\right) \subset\left(\mathbb{U}^{\prime}\right)^{[3]} \subset \mathbb{U} \otimes\left(\mathbb{U}^{\prime}\right)^{[2]} .
$$

(b) The ring $\mathbb{F}^{\prime}$ is a $\mathbb{U}^{[2]}$-algebra. It is the localization of $\mathbb{F}_{+}^{\prime}$ at the multiplicative set generated by $c_{\omega_{n}}$, a central element. The assignment $c_{i j} \mapsto t_{i j}$ is an isomorphism from $\mathbb{F}_{+}^{\prime}$ to the algebra generated by $\left\{t_{i j}\right\}$ with the defining relations $R_{21}^{q} T_{13} R_{12}^{q} T_{23}=T_{23} R_{21}^{q} T_{13} R_{12}^{q}$ where $T=\sum_{i, j} e_{i j} \otimes t_{i j}$.

Proof. The maps $\kappa, \bar{\kappa}$ are (adU)-algebra homomorphisms $\mathbb{F}^{\prime} \rightarrow \mathbb{U}^{\prime}$ by 1.4.1(b). The invertibility of $\kappa$ is proved in [J2], [BS]. The inclusion $\Delta\left(\mathbb{U}^{\prime}\right) \subset\left(\mathbb{U}^{\prime}\right)^{[2]}$ follows from the equalities $\kappa\left(\mathbb{F}^{\prime}\right)=\mathbb{U}^{\prime}$ and $\Delta \kappa(f)=\varpi_{2}\left(\kappa f_{1} \otimes \kappa f_{2}\right)$; see above and 1.3.3(c). The inclusion $\left(\mathbb{U}^{\prime}\right)^{[2]} \subset \mathbb{U} \otimes \mathbb{U}^{\prime}$ follows from the formula for $\varpi_{2}$ in section A.1. Part $(b)$ is proved in [DM, prop. 4.11].

Let $\mathbb{U}_{\pi} \subset \mathbb{U}$ be the $\mathcal{K}$-subalgebra generated by the elements $\iota\left(f_{i}\right), k_{\lambda}, e_{j}$ with $\lambda_{1}=0, j \neq 1$. Let $\mathbb{U}_{\pi}^{\prime}=\mathbb{U}_{\pi} \cap \mathbb{U}^{\prime}$. Finally, let $\mathbb{U}_{\tilde{\pi}} \subset \mathbb{U}$ be the Hopf subalgebra generated by $\mathbb{U}_{0}$ and $\mathbb{U}_{\pi}$. Both $\mathbb{U}_{\pi}, \mathbb{U}_{\pi}^{\prime}$ are normal left coideal subalgebras of $\mathbb{U}_{\tilde{\pi}}$.

Set $\mathbb{V}=\mathbb{U}^{\prime} / \mathbb{I}_{V}$ where $\mathbb{I}_{V}$ is the right ideal generated by $\left(\mathbb{U}_{\pi}^{\prime}\right)^{\varepsilon}=\mathbb{U}_{\pi}^{\prime} \cap \mathbb{U}^{\varepsilon}$. The adjoint $\mathbb{U}_{\tilde{\pi}^{-a c t i o n}}$ on $\mathbb{U}^{\prime}$ factors to $\mathbb{V}$. It is locally finite. Let $\mathbb{V}_{+}$be the image of

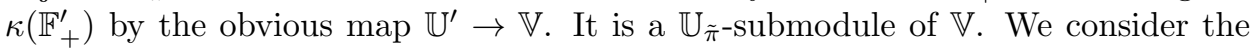
restriction of this action to the $\mathcal{K}$-subalgebra $\iota\left(\mathbb{U}_{\pi}\right) \subset \mathbb{U}_{\tilde{\pi}}$.

For any right $\mathbb{U}_{\pi}$-module $V$ we abbreviate $V^{\pi}=V^{\mathbb{U}_{\pi}}$. By 1.4.1 $(d)$ the subset $\mathbb{F}^{\pi} \subset \mathbb{F}$ is a $\mathbb{U}$-subalgebra for the standard left $\mathbb{U}$-action. As a $\mathcal{K}$-algebra it is generated by the set $\left\{c_{1 i}\right\}$. Also, let ${ }^{\pi} \mathbb{F} \subset \mathbb{F}$ be the $\mathbb{U}$-subalgebra generated by the set $\left\{c_{i 1}\right\}$ with the contragredient left action, i.e., the right $\mathbb{U}$-action twisted by the antipode. The following is proved in section A.4.

1.7.2. Lemma. (a) The $\mathcal{K}$-algebras $\mathbb{U}_{\pi}^{\prime},\left(\mathbb{U}_{\pi}^{\prime}\right)^{\varepsilon}$ are generated by the subsets $\left\{\kappa \iota\left(c_{i j}\right)\right.$; $j \neq 1\},\left\{\kappa \iota\left(c_{i j}\right)-\delta_{i j} ; j \neq 1\right\}$, respectively.

(b) There is an $\iota\left(\mathbb{U}_{\pi}\right)$-module isomorphism $\mathbb{V}_{+} \simeq \pi \mathbb{F}$. The $\iota\left(\mathbb{U}_{\pi}\right)$-module $\mathbb{V}$ is isomorphic to the localization of ${ }^{\pi} \mathbb{F}$ with respect to the multiplicative set generated by $c_{11}$.

\subsubsection{Remarks.}

(a) The Hopf algebra $\mathbb{U}$ is not quasi-triangular, but $\mathbb{F}$ is coquasi-triangular. For $V, W \in \mathcal{O}(\mathbb{U})$ the universal $R$-matrix maps the element $v \otimes w \in V \otimes W$ to a well-defined element

$$
\sum_{s}\left(r_{s}^{+} \triangleright v\right) \otimes\left(r_{s}^{-} k_{|v|} \triangleright w\right)=\sum_{s}\left(r_{s}^{+} k_{|w|} \triangleright v\right) \otimes\left(r_{s}^{-} \triangleright w\right),
$$

for some homogeneous element $r_{s}^{ \pm} \in \mathbb{U}_{ \pm}$. If $f \in \mathbb{F}$ is homogeneous of weight $\lambda$ relative to $\triangleright$, then $R^{+}(f)=\sum_{s}\left(f: r_{s}^{+}\right) r_{s}^{-} k_{\lambda}$ and $R^{-} \iota(f)=$ $\sum_{s}\left(f: r_{s}^{-}\right) r_{s}^{+} k_{\lambda}$.

(b) The U-algebra $\mathbb{F}^{\pi}$ is isomorphic to $\bigoplus_{m \geqslant 0} V\left(m \omega_{1}\right)$ with the Cartan multiplication rule. We have also ${ }^{\pi} \mathbb{F}=\bigoplus_{m \geqslant 0} V\left(m \omega_{1}\right)^{\circ}$ as a $\mathbb{U}$-module. 
(c) Set $\mathbb{F}_{i}^{\pi}=\left(\mathbb{F}_{i}\right)^{\pi}$ and $\mathbb{F}_{*}^{\pi}=\left(\mathbb{F}_{*}\right)^{\pi}$. For any $\mathbb{F}^{\pi}$-module $\mathbb{V}$ we write

$$
V_{i}=\mathbb{F}_{i}^{\pi} \otimes_{\mathbb{F} \pi} V, \quad V_{*}=\mathbb{F}_{*}^{\pi} \otimes_{\mathbb{F} \pi} V .
$$

The $\mathcal{K}$-algebra $\mathbb{F}_{*}^{\pi}$ is the quantum torus generated by the invertible elements $c_{1 i}^{ \pm 1}, i=1,2, \ldots, n$, modulo the relations $c_{1 i} c_{1 j}=q c_{1 j} c_{1 i}$ if $i>j$. For each weight $\lambda=\left(\lambda_{1}, \lambda_{2}, \ldots, \lambda_{n}\right)$ we put $c_{1 \lambda}=\left(c_{1 n}\right)^{\lambda_{n}} \cdots\left(c_{12}\right)^{\lambda_{2}}\left(c_{11}\right)^{\lambda_{1}}$. Let $x_{\lambda}, y_{\lambda} \in \operatorname{End}\left(\mathbb{F}_{*}^{\pi}\right)$ be given by $y_{\lambda}\left(c_{1 \mu}\right)=q^{\lambda \cdot \mu} c_{1 \mu}$ and $x_{\lambda}\left(c_{1 \mu}\right)=c_{1, \lambda+\mu}$ for each $\mu$. We abbreviate $y_{i}=y_{\varepsilon_{i}}$ and $x_{i}=x_{\varepsilon_{i}}$. Set $\tilde{\lambda}=\sum_{j \neq 1} \lambda_{j} \omega_{j-1}$. The elements $\partial_{\triangleright}\left(\dot{e}_{j}\right), \partial_{\triangleright}\left(\dot{f}_{j}\right), \partial_{\triangleright}\left(k_{\lambda}\right)$ and $\ell\left(c_{1 \lambda}\right) \partial_{\triangleright}\left(k_{\tilde{\lambda}}\right)^{-1}$ of $\mathbb{D}_{\triangleright}$ act on $\mathbb{F}_{*}^{\pi}$ as the operators

$$
x_{j} x_{j+1}^{-1}\left(y_{j+1}-y_{j+1}^{-1}\right), x_{j}^{-1} x_{j+1}\left(y_{j}-y_{j}^{-1}\right), y_{\lambda}, x_{\lambda} .
$$

(d) We have $\mathbb{U} \subset \mathbb{F}^{*}$, i.e., the natural pairing $\mathbb{F} \times \mathbb{U} \rightarrow \mathcal{K}$ is nondegenerate.

(e) The $\mathcal{K}$-algebra $\mathbb{F}$ is a quantum analogue of the function algebra $\mathcal{O}(G)$. Let $\bar{c}_{\lambda}, \bar{c}_{i j} \in \mathcal{O}(G)$ be the functions corresponding to the elements $c_{\lambda}, c_{i j}$. We write again $\Sigma=\left\{\bar{c}_{\lambda} ; \lambda \in X_{+}\right\}$, a subset of $\mathcal{O}(G)$. We have $\Sigma^{-1} \mathcal{O}(G)=$ $\mathcal{O}\left(G_{\Sigma}\right)$ with $G_{\Sigma}=U_{+} H U_{-}$the open Bruhat cell of $G$.

(f) We will abbreviate $\Sigma=\kappa(\Sigma)$, a multiplicative set in $\mathbb{U}^{\prime}$. Let $\mathbb{U}_{\Sigma}^{\prime}$ be the corresponding quotient ring.

From now on we use the following conventions:

- to simplify we omit the terms " $k_{\lambda}$ " in the formulas for the $R$-matrix,

- given an element $u$ of $\mathbb{U}$ or $\mathbb{U}^{\circ}$ the expression $u_{1} \otimes u_{2}$ will always denote the coproduct in $\mathbb{U}$. So we write $u_{2} \otimes u_{1}$ for the coproduct in $\mathbb{U}^{\circ}$.

1.8. QDO on $G$. The Hopf algebras $\mathbb{U}, \mathbb{U}^{\circ}$ act on the $\mathcal{K}$-algebra $\mathbb{F}$ via the natural left action and the contragredient left action, respectively. Let $\mathbb{D}_{\triangleright}=\mathbb{F} \sharp \mathbb{U}$ and $\mathbb{D}_{\triangleleft}=\mathbb{F} \sharp \mathbb{U}^{\circ}$. Both are $\mathbb{U}^{e}$-algebras by $1.4 .3(a)$. In section 1.4 we have defined inclusions

$$
\partial_{\triangleright}: \mathbb{U} \rightarrow \mathbb{D}_{\triangleright}, \quad \partial_{\triangleright}: \mathbb{U}^{\circ} \rightarrow \mathbb{D}_{\triangleleft} .
$$

To avoid confusion we will use the symbol $\partial_{\triangleright}$ for the first map and we will use the composed morphism

$$
\partial_{\triangleleft}=\partial_{\triangleright} \circ \bar{\iota}: \mathbb{U}_{\diamond} \rightarrow \mathbb{D}_{\triangleleft}
$$

rather than the second map. To simplify the notation we may write $\mathbb{U}$ instead of $\mathbb{U}_{\text {。 }}$. So we view $\partial_{\triangleleft}$ as a map $\mathbb{U} \rightarrow \mathbb{D}_{\triangleleft}$. Recall that

$$
\begin{aligned}
& \partial_{\triangleright}(u) \ell(f)=\ell\left(u_{1} \triangleright f\right) \partial_{\triangleright}\left(u_{2}\right), \\
& \partial_{\triangleleft}(u) \ell(f)=\ell\left(f \triangleleft u_{1}\right) \partial_{\triangleleft}\left(u_{2}\right) .
\end{aligned}
$$

Note that $\mathbb{U}^{\prime} \subset \mathbb{U}$ is a normal left coideal subalgebra by 1.7.1(a). Let $\mathbb{D} \subset \mathbb{D}_{\triangleright}$ be the $\mathbb{U}^{e}$-subalgebra generated by $\ell(\mathbb{F}), \partial_{\triangleright}\left(\mathbb{U}^{\prime}\right)$. It is isomorphic to the subalgebra of $\mathbb{D}_{\triangleleft}$ generated by $\ell(\mathbb{F}), \partial_{\triangleleft}\left(\mathbb{U}^{\prime}\right)$. The $\mathbb{U}^{e}$-action on $\mathbb{D}$ is given by

$$
\begin{aligned}
& \left(u \otimes u^{\prime}\right) \triangleright\left(\ell(f) \partial_{\triangleright}(v)\right)=\ell\left(u_{1}^{\prime} \triangleright f \triangleleft \iota(u)\right) \partial_{\triangleright}\left(\operatorname{ad} u_{2}^{\prime}\right)(v), \\
& \left(u \otimes u^{\prime}\right) \triangleright\left(\ell(f) \partial_{\triangleleft}(v)\right)=\ell\left(u^{\prime} \triangleright f \triangleleft \iota\left(u_{2}\right)\right) \partial_{\triangleleft}\left(\operatorname{ad} u_{1}\right)(v) .
\end{aligned}
$$

The adjoint $\mathbb{U}$-action on $\mathbb{D}$ is given by

$$
\begin{aligned}
& (\operatorname{ad} u)\left(\ell(f) \partial_{\triangleright}(v)\right)=\ell\left(\operatorname{ad} u_{1}\right)(f) \partial_{\triangleright}\left(\operatorname{ad} u_{2}\right)(v), \\
& (\operatorname{ad} u)\left(\ell(f) \partial_{\triangleleft}(v)\right)=\ell\left(\operatorname{ad} u_{2}\right)(f) \partial_{\triangleleft}\left(\operatorname{ad} u_{1}\right)(v) .
\end{aligned}
$$


Let $\mathbb{D}_{+} \subset \mathbb{D}$ be the subalgebra generated by $\ell\left(\mathbb{F}_{+}\right)$and $\partial_{\triangleright} \kappa\left(\mathbb{F}_{+}\right)$. The following is proved in section A.5.

1.8.2. Proposition. (a) The ring $\mathbb{D}_{\triangleright}$ is an $I D$.

(b) The basic $\mathbb{D}_{\triangleright}$-module is faithful and $\mathbb{U}^{e}$-equivariant.

(c) There are $Q M M \partial_{\triangleleft}: \mathbb{U}^{\prime} \rightarrow \mathbb{D}$ and $\partial_{\triangleright}: \mathbb{U}^{\prime} \rightarrow \mathbb{D}$.

(d) The $\mathcal{K}$-linear map $\mathbb{F} \otimes \mathbb{U}^{\prime} \rightarrow \mathbb{D}, f \otimes u \mapsto \ell(f) \partial_{\triangleleft}(u)$ is invertible.

The adjoint $\mathbb{U}$-action on $\mathbb{D}$ is not a Hopf algebra action. Thus it is convenient to introduce the following $\mathcal{K}$-subalgebras:

$$
\mathbb{D}^{\prime}=\mathbb{F}^{\prime} \sharp \mathbb{U}^{\prime}, \quad \mathbb{D}_{\triangleright}^{\prime}=\mathbb{F}^{\prime} \sharp \mathbb{U} \subset \mathbb{F}^{\prime} \sharp \mathbb{U}^{[2]} .
$$

Here $\mathbb{U}^{\prime}, \mathbb{U}$ embed into $\mathbb{U}^{[2]}$ as in 1.3.3(a). Both are $\mathbb{U}^{[2]}$-algebras. Note that $\mathbb{D}_{\triangleright}^{\prime} \simeq \mathbb{D}_{\triangleleft}$ by 1.4.3(b). The adjoint $\mathbb{U}$-action is given by

$$
(\operatorname{ad} u)\left(\ell(f) \partial_{\triangleright}(v)\right)=\ell\left(\operatorname{ad} u_{1}\right)(f) \partial_{\triangleright}\left(\operatorname{ad} u_{2}\right)(v) .
$$

To understand the semi-classical analogue of $\mathbb{D}^{\prime}$ it is useful to write a presentation in matrix form. Given formal symbols $\ell_{i j}, \ell_{i j}^{\prime}$ set $L=\sum_{i, j} e_{i j} \otimes \ell_{i j}$ and $L^{\prime}=$ $\sum_{i, j} e_{i j} \otimes \ell_{i j}^{\prime}$. Let $\ell_{\omega_{n}}, \ell_{\omega_{n}}^{\prime}$ be the quantum determinants of $L, L^{\prime}$. Let $\mathbb{D}_{+}^{\prime}$ be the $\mathcal{K}$-algebra generated by $\left\{\ell_{i j}, \ell_{i j}^{\prime}\right\}$ with the relations

$$
\begin{aligned}
& R_{21}^{q} L_{13} R_{12}^{q} L_{23}=L_{23} R_{21}^{q} L_{13} R_{12}^{q}, \\
& R_{21}^{q} L_{13}^{\prime} R_{12}^{q} L_{23}^{\prime}=L_{23}^{\prime} R_{21}^{q} L_{13}^{\prime} R_{12}^{q}, \\
& R_{21}^{q} L_{13} R_{12}^{q} L_{23}^{\prime}=L_{23}^{\prime} R_{21}^{q} L_{13}\left(R_{21}^{q}\right)^{-1} .
\end{aligned}
$$

The following is proved in section A.5.

1.8.3. Proposition. (a) There is a $Q M M \partial_{2}:\left(\mathbb{U}^{\prime}\right)^{[2]} \rightarrow \mathbb{D}^{\prime}$.

(b) The elements $\ell_{\omega_{n}}, \ell_{\omega_{n}}^{\prime}$ generate a denominator set of $\mathbb{D}_{+}^{\prime}$ whose quotient ring is isomorphic to $\mathbb{D}^{\prime}$.

1.8.4. Remark. The ring $\mathbb{D}_{\triangleleft}$ satisfies properties similar to 1.8.2. For future use, observe that $\partial_{\triangleright}\left(\mathbb{U}^{\prime}\right) \subset\left(\mathbb{D}_{\triangleright}\right)^{\triangleleft \mathbb{U}}, \partial_{\triangleleft}\left(\mathbb{U}^{\prime}\right) \subset\left(\mathbb{D}_{\triangleright}\right)^{\mathbb{U} \triangleright}$ and that $\left(\mathbb{D}_{\triangleright}\right)^{\triangleleft \mathbb{U}}$, $\left(\mathbb{D}_{\triangleright}\right)^{\mathbb{U} \triangleright}$ centralize each other in $\mathbb{D}_{\triangleright}$. See section A.5 for details.

1.9. QDO on $\mathbb{P}^{n-1}$. In this section we study several versions of the ring of QDO on $\mathbb{P}^{n-1}$. First, write $\mathbb{R}=\mathbb{D} / \mathbb{I}_{R}$ where $\mathbb{I}_{R}$ is the left ideal generated by $\partial_{\triangleleft}\left(\mathbb{U}_{\pi}^{\prime}\right)^{\varepsilon}$. It is a $\mathbb{U}_{\tilde{\pi}}^{\circ} \otimes \mathbb{U}$-equivariant $\mathbb{D}$-module. Let $\mathbb{D}^{\pi} \subset \mathbb{D}$ be the set of right $\mathbb{U}_{\pi^{-}}$-invariant elements. It is a $\mathbb{U}$-algebra by 1.4.1(d). By 1.5.2 the subset $\mathbb{R}^{\pi} \subset \mathbb{R}$ of right $\mathbb{U}_{\pi^{-}}$ invariant elements is also a $\mathbb{U}$-algebra. It is the first analogue of DO on $\mathbb{P}^{n-1}$. For technical reasons we need two other different versions of the ring of QDO on $\mathbb{P}^{n-1}$.

The $\mathcal{K}$-linear isomorphism $\ell \otimes \partial_{\triangleleft}: \mathbb{F} \otimes \mathbb{U}^{\prime} \rightarrow \mathbb{D}$ factors to an isomorphism

$$
\ell \otimes \partial_{\triangleleft}: \mathbb{F} \otimes \mathbb{V} \rightarrow \mathbb{R}
$$

Let $\mathbb{R}_{+}$be the image of $\mathbb{F} \otimes \mathbb{V}_{+}$by this isomorphism. It is a $\mathbb{U}_{\tilde{\pi}}^{\circ} \otimes \mathbb{U}_{\text {-submodule of }}$ $\mathbb{R}$. Let $\mathbb{R}_{+}^{\pi} \subset \mathbb{R}_{+}$be the set of $\mathbb{U}_{\pi}$-invariant elements, a $\mathbb{U}$-submodule again. Here we identify $\mathbb{U}_{\pi}$ with the algebra $\mathbb{U}_{\pi} \otimes 1$. It is the second analogue of DO on $\mathbb{P}^{n-1}$.

Let $\mathbb{D}_{\triangleright}^{\pi} \subset \mathbb{D}_{\triangleright}$ be the set of right $\mathbb{U}_{\pi}$-invariant elements. The basic representation of $\mathbb{D}_{\triangleright}$ on $\mathbb{F}$ factors to a representation of $\mathbb{D}_{\triangleright}^{\pi}$ on $\mathbb{F}^{\pi}$. Let $\mathbb{R}_{\triangleright}^{\pi} \subset \operatorname{End}\left(\mathbb{F}^{\pi}\right)$ be the $\mathcal{K}$-subalgebra generated by the action of $\mathbb{D}_{\triangleright}^{\pi}$ on $\mathbb{F}^{\pi}$. It is the third analogue of DO on $\mathbb{P}^{n-1}$. 
We also use some quotient rings. The elements $\ell\left(c_{1 i}\right), \partial_{\triangleright}\left(k_{\lambda}\right), i \in I, \lambda \in 2 X_{+}$ belong to $\mathbb{D}^{\pi}$. Hence they map into $\mathbb{R}^{\pi}$. Let $\mathbb{R}_{\Sigma}^{\pi}$ be the quotient ring obtained by inverting all of these elements.

The following is proved in section A.6.

1.9.1. Proposition. (a) The algebra $\mathbb{R}_{\triangleright}^{\pi}$ is an ID and a $\mathbb{U}$-algebra. The quotient ring $\mathbb{R}_{\triangleright, *}^{\pi}$ is a quantum torus.

(b) The algebra $\mathbb{R}^{\pi}$ is an ID and $a \mathbb{U}$-subalgebra of $\operatorname{End}\left(\mathbb{F}^{\pi}\right)$. The quotient ring $\mathbb{R}_{\Sigma}^{\pi}$ is a quantum torus.

(c) The $\mathcal{K}$-subspace $\mathbb{R}_{+}^{\pi} \subset \mathbb{R}^{\pi}$ is a $\mathbb{U}$-subalgebra. There is an isomorphism of $\mathbb{U}$-equivariant $\mathbb{F}^{\pi}$-modules $\mathbb{F}^{\pi} \otimes \pi \mathbb{F} \rightarrow \mathbb{R}_{+}^{\pi}$.

\subsubsection{Remarks.}

(a) In 1.9.1(c) we have equipped $\mathbb{F}^{\pi} \otimes^{\pi} \mathbb{F}$ with the tensor product of the left $\mathbb{U}$ action on $\mathbb{F}^{\pi}$ and the contragredient left $\mathbb{U}$-action on ${ }^{\pi} \mathbb{F}$. The isomorphism $\mathbb{F}^{\pi} \otimes{ }^{\pi} \mathbb{F} \rightarrow \mathbb{R}_{+}^{\pi}$ is given by $f \otimes f^{\prime} \mapsto \ell\left(f \iota\left(f_{1}^{\prime}\right)\right) \partial_{\triangleleft} \kappa\left(f_{2}^{\prime}\right)$.

(b) The left action of $k_{\omega_{n}}$ yields the $\mathbb{Z}$-grading on $\mathbb{R}^{\pi}$ given by

$$
\operatorname{deg}(x)=d \Longleftrightarrow k_{\omega_{n}} \triangleright x=q^{d} x .
$$

The subalgebra $\mathbb{R}_{+}^{\pi}$ is also $\mathbb{Z}$-graded. The elements $c_{1 i} \otimes 1,1 \otimes c_{i 1} \in \mathbb{F}^{\pi} \otimes^{\pi} \mathbb{F}$ have degree $1,-1$.

(c) The proof of 1.9.1(b) uses the surjection $\mathbb{D}_{i}^{\pi} \rightarrow \mathbb{R}_{i}^{\pi}$. The natural map $\mathbb{D}^{\pi} \rightarrow \mathbb{R}^{\pi}$ is not surjective. Indeed, the left action of $k_{\omega_{n}}$ yields a $\mathbb{Z}_{+^{-}}$ grading on $\mathbb{D}^{\pi}$ as in part (b) above. The canonical map $\mathbb{D}^{\pi} \rightarrow \mathbb{R}^{\pi}$ preserves the grading, so it cannot be surjective.

$(d)$ The map $\partial_{\triangleright}: \mathbb{U}^{\prime} \rightarrow \mathbb{D}$ factors to $\mathbb{U}^{\prime} \rightarrow \mathbb{R}^{\pi}$.

1.10. QDO on $G \times \mathbb{P}^{n-1}$. First, we introduce a ring of QDO on $G \times G$. The Hopf algebra $\mathbb{H}=\mathbb{U}^{\circ} \otimes \mathbb{U}^{[3]}$ is equivalent to $\left(\mathbb{U}^{e}\right)^{\otimes 2}$. The tensor square $\mathbb{F}^{\otimes 2}$ is a $\left(\mathbb{U}^{e}\right)^{\otimes 2}$-algebra such that

$$
\left(u \otimes v \otimes u^{\prime} \otimes v^{\prime}\right) \triangleright\left(f \otimes f^{\prime}\right)=(v \triangleright f \triangleleft \iota(u)) \otimes\left(v^{\prime} \triangleright f^{\prime} \triangleleft \iota\left(u^{\prime}\right)\right) .
$$

So we may twist the multiplication in $\mathbb{F}^{\otimes 2}$ to get an $\mathbb{H}$-algebra $\mathbb{G}$. Now we define some subalgebra of the smash-product $\mathbb{G} \sharp \mathbb{H}$. Set $\mathbb{H}^{\prime}=\iota\left(\mathbb{U}^{\prime}\right) \otimes\left(\mathbb{U}^{\prime}\right)^{[3]}$. The inclusions

$$
\begin{aligned}
& a: \mathbb{U}^{[2]} \rightarrow \mathbb{H}, u \mapsto 1 \otimes u \otimes 1, \\
& b: \mathbb{U}^{\circ} \rightarrow \mathbb{H}, u \mapsto u \otimes 1^{3}, \\
& c: \mathbb{U} \rightarrow \mathbb{H}, u \mapsto 1 \otimes \Delta^{2}(u)
\end{aligned}
$$

restrict to algebra embeddings of $\left(\mathbb{U}^{\prime}\right)^{[2]}, \iota\left(\mathbb{U}^{\prime}\right)$ and $\mathbb{U}^{\prime}$ into $\mathbb{H}^{\prime}$. Note that $\left(\mathbb{U}^{\prime}\right)^{[2]} \subset \mathbb{H}$ is a normal left coideal subalgebra by $1.3 .3(a)$. Thus there is an $\mathbb{H}$-algebra $\mathbb{E}=$ $\mathbb{G} \sharp\left(\mathbb{U}^{\prime}\right)^{[2]}$. Consider the following linear maps:

$$
\begin{aligned}
& \gamma: \mathbb{D} \rightarrow \mathbb{E}, \quad \ell(f) \partial_{\triangleright}(v) \mapsto \ell(f \otimes 1) \partial_{\triangleright} \varpi_{2}(v \otimes 1), \\
& \gamma^{\prime}: \mathbb{D}^{\prime} \rightarrow \mathbb{E}, \quad \ell\left(f^{\prime}\right) \partial_{\triangleright}\left(u^{\prime}\right) \mapsto \ell\left(1 \otimes f^{\prime}\right) \partial_{\triangleright} \varpi_{2}\left(1 \otimes u^{\prime}\right), \\
& \psi: \mathbb{D} \otimes \mathbb{D}^{\prime} \rightarrow \mathbb{E}, \quad d \otimes d^{\prime} \mapsto \gamma(d) \gamma^{\prime}\left(d^{\prime}\right) .
\end{aligned}
$$

The following is proved in section A.7.

1.10.1. Proposition. (a) The maps $\gamma: \mathbb{D} \rightarrow \mathbb{E}$ and $\gamma^{\prime}: \mathbb{D}^{\prime} \rightarrow \mathbb{E}$ are algebra homomorphisms. 
(b) The map $\psi: \mathbb{D} \otimes \mathbb{D}^{\prime} \rightarrow \mathbb{E}$ is invertible.

(c) The $\mathbb{U}$-actions on $\mathbb{E}$ associated with $b, c$ are given respectively by

$u \triangleright \psi\left(d \otimes d^{\prime}\right)=\psi\left((d \triangleleft \iota(u)) \otimes d^{\prime}\right), \quad u \triangleright \psi\left(d \otimes d^{\prime}\right)=\psi\left(\left(u_{1} \triangleright d\right) \otimes\left(\operatorname{ad} u_{2}\right)\left(d^{\prime}\right)\right)$.

(d) The basic representation of $\mathbb{E}$ on $\mathbb{G}$ is faithful.

(e) There is a quantum moment map $\partial_{3}: \mathbb{H}^{\prime} \rightarrow \mathbb{E}$.

Let $\partial_{a}, \partial_{b}, \partial_{c}$ be the maps composed of $\partial_{3}$ and $a, b, c$.

Let $\mathbb{G}_{i}, \mathbb{G}_{*}$ be the rings of quotients of $\mathbb{G}$ relative to the multiplicative sets generated by $c_{1 i} \otimes 1$ and $\left\{c_{1 i} \otimes 1\right\}$. Let $\mathbb{G}^{\pi} \subset \mathbb{G}$ be the set of $\mathbb{U}_{\pi}$-invariant elements for the action associated with the map $b$. It is a subalgebra of $\mathbb{G}$. We define $\mathbb{E}_{i}, \mathbb{E}_{*}$ and $\mathbb{E}^{\pi}$ in the same way.

Now we can introduce the ring of QDO on $G \times \mathbb{P}^{n-1}$. It is one of the main objects of this paper. Put $\mathbb{S}=\mathbb{E} / \mathbb{I}_{S}$ where $\mathbb{S}$ is the left ideal generated by $\partial_{b} \iota\left(\mathbb{U}_{\pi}^{\prime}\right)^{\varepsilon}$. The quantum reduction of $\mathbb{E}$ relative to $\partial_{b}$ yields the algebra

$$
\mathbb{S}^{\pi}=\mathbb{E} / / \iota\left(\mathbb{U}_{\pi}^{\prime}\right) .
$$

The $\mathbb{U}$-action on $\mathbb{E}$ associated with the map $c$ is called the adjoint action. It factors to a $\mathbb{U}$-action on $\mathbb{S}^{\pi}$. The following is proved in section A.7.

1.10.3. Proposition. (a) The map $\psi$ factors to linear isomorphisms $\mathbb{R} \otimes \mathbb{D}^{\prime} \rightarrow \mathbb{S}$, $\mathbb{R}^{\pi} \otimes \mathbb{D}^{\prime} \rightarrow \mathbb{S}^{\pi}$.

(b) The ring $\mathbb{S}^{\pi}$ is an $I D$. The maps $\partial_{a}$, $\partial_{c}$ factor to $\partial_{a}:\left(\mathbb{U}^{\prime}\right)^{[2]} \rightarrow \mathbb{S}^{\pi}, \partial_{c}$ : $\mathbb{U}^{\prime} \rightarrow \mathbb{S}^{\pi}$. Both are QMM.

\subsubsection{Remarks.}

(a) The relations for $\mathbb{D}_{+}^{\prime}$ in 1.8 .3 are homogeneous. We equip $\mathbb{D}^{\prime}$ with the $\mathbb{Z}$-grading such that $\operatorname{deg}\left(\ell_{i j}\right)=\operatorname{deg}\left(\ell_{i j}^{\prime}\right)=1$.

(b) We equip $\mathbb{D}$ with the $\mathbb{Z}$-grading such that $\operatorname{deg}\left(\ell\left(c_{i j}\right)\right)=1$ and $\operatorname{deg}\left(\partial_{\triangleright} \kappa\left(c_{i j}\right)\right)$ $=0$, compare 1.9.2(c).

(c) We equip $\mathbb{E}$ with the $\mathbb{Z}$-grading such that the linear map $\psi: \mathbb{D} \otimes \mathbb{D}^{\prime} \rightarrow \mathbb{E}$ is homogeneous of degree 0 . This grading is preserved by the $\mathbb{U}$-action associated with the maps $b, c$. Further, $\partial_{b}, \partial_{c}$ map into the homogeneous component of degree 0 by A.7.4(b) and A.5.3.

(d) We equip $\mathbb{S}^{\pi}$ with the $\mathbb{Z}$-grading such that the linear map $\psi: \mathbb{R}^{\pi} \otimes \mathbb{D}^{\prime} \rightarrow \mathbb{S}^{\pi}$ is homogeneous of degree 0 . Here $\mathbb{R}^{\pi}, \mathbb{D}^{\prime}$ are given the gradings in 1.9.2(b), 1.10.4(a). In other words, the grading on $\mathbb{S}^{\pi}$ is given by the action of $k_{\omega_{n}}$ associated with the map $c$; see 1.9.2(b).

1.11. The deformed Harish-Chandra homomorphism. The purpose of this section is to define a particular homomorphism between two different rings of QDO. Our construction uses intertwiners. First we recall what intertwiners are. For $V, W \in \boldsymbol{M o d}(\mathbb{U})$ there is a representation of $\mathbb{U}$ on $\operatorname{Hom}(V, W \otimes V)$ given by

$$
(u \triangleright f)(v)=u_{1} \triangleright f\left(\iota\left(u_{2}\right) \triangleright v\right) .
$$

The $\mathbb{U}$-invariant elements are the $\mathbb{U}$-linear maps $V \rightarrow W \otimes V$.

We define the adjoint representation of $\mathbb{U}$ on $\operatorname{Hom}(\mathbb{U}, W)$ as follows. For any $u \in$ $\mathbb{U}, g \in \operatorname{Hom}(\mathbb{U}, W)$ the map $(\operatorname{ad} u) g \in \operatorname{Hom}(\mathbb{U}, W)$ is given by $x \mapsto u_{1} \triangleright g\left(\operatorname{ad}_{r}\left(u_{2}\right) x\right)$. This action preserves the subspace $W \otimes \mathbb{U}^{*} \subset \operatorname{Hom}(\mathbb{U}, W)$ of finite rank operators. Further, the adjoint action on $W \otimes \mathbb{U}^{*}$ is given by

$$
(\operatorname{ad} u)(w \otimes f)=\left(u_{1} \triangleright w\right) \otimes\left(\operatorname{ad} u_{2}\right) f .
$$


Now, assume that $V \in \boldsymbol{O}(\mathbb{U})$. We have an embedding of $\mathbb{U}$-modules

$$
\operatorname{Hom}(V, W \otimes V) \subset \operatorname{Hom}(\mathbb{U}, W)
$$

taking $g$ to the map

$$
u \mapsto \sum_{i}\left(\operatorname{id}_{W} \otimes \varphi_{i}: g\left(\iota(u) k_{2 \rho} v_{i}\right)\right) .
$$

Here $\left(v_{i}\right),\left(\varphi_{i}\right)$ are dual bases of $V, V^{*}$. Let $I(W, V)$ be the image of this map. Put

$$
W \hat{\otimes} \mathbb{F}=\sum_{V \in \mathcal{O}(\mathbb{U})} I(W, V) .
$$

This is a $\mathbb{U}$-module for the adjoint action. Taking invariants commutes with direct limits. Thus the set of $\mathbb{U}$-invariant elements in $W \hat{\otimes} \mathbb{F}$ is the sum of the spaces of intertwiner operators $V \rightarrow W \otimes V$.

Now we can define the deformed Harish-Chandra homomorphism. Before doing that we must define a new ring of QDO. We will see later that this ring, denoted $\mathbb{T}^{0}$, is indeed a quantum analogue of the ring of differential operators on the punctual Hilbert scheme of $\mathbb{A}^{2}$. Fix a unit $t \in \mathcal{K}$. Set $\bar{\chi}=\chi \circ \iota$, where $\chi$ is the unique $\mathcal{K}$-algebra homomorphism

$$
\chi: \mathbb{U} \rightarrow \mathcal{K}, \quad k_{\lambda} \mapsto q^{\lambda \cdot \omega_{n}} t^{-\lambda \cdot \omega_{n}} .
$$

Consider the quantum reduction relative to $\partial_{c}$,

$$
\mathbb{T}^{0}=\mathbb{S}^{\pi} / / \bar{\chi} \mathbb{U}^{\prime}
$$

It is a $\mathcal{K}$-algebra. Note that $\mathbb{T}^{0}$ is also the quantum reduction relative to $\partial_{b}, \partial_{c}$,

$$
\mathbb{T}^{0}=\mathbb{E} / / \varepsilon \otimes \bar{\chi}\left(\iota\left(\mathbb{U}_{\pi}^{\prime}\right) \otimes \mathbb{U}^{\prime}\right) .
$$

The $\mathcal{K}$-algebra $\mathbb{T}^{0}$ is equipped with two natural algebra homomorphisms. Indeed, recall that $\Delta\left(\mathbb{U}^{\prime}\right) \subset\left(\mathbb{U}^{\prime}\right)^{[2]}$ by 1.7.1(a). Thus we have the maps

$$
\begin{aligned}
& z^{\prime}=\gamma^{\prime} \circ \ell: \mathbb{F}^{\prime} \rightarrow \mathbb{E}, \\
& z=\partial_{a} \circ \Delta: \mathbb{U}^{\prime} \rightarrow \mathbb{E} .
\end{aligned}
$$

They yield algebra homomorphisms

$$
\begin{aligned}
& z^{\prime}: Z\left(\mathbb{F}^{\prime}\right) \rightarrow \mathbb{T}^{0}, \\
& z: Z\left(\mathbb{U}^{\prime}\right) \rightarrow \mathbb{T}^{0} .
\end{aligned}
$$

We will drop the symbols $z, z^{\prime}$ when there is no danger of confusion. Indeed, $Z\left(\mathbb{F}^{\prime}\right) \subset \mathbb{F}^{\prime}$ is the set of $\mathbb{U}$-invariant elements for the adjoint action by 1.7.1(a). Thus $z^{\prime}$ maps into $\mathbb{T}^{0}$ by definition of $\gamma^{\prime}$. Similarly, $z$ maps into $\mathbb{T}^{0}$ because $\partial_{a}\left(u_{1} \otimes u_{2}\right)$ commutes with $\partial_{c}(v)$ for each $u \in Z\left(\mathbb{U}^{\prime}\right), v \in \mathbb{U}^{\prime}$. For future use, recall that there is a unique $\mathcal{K}$-algebra isomorphism

$$
\Omega: \mathbb{U}_{0}^{\Sigma_{n}} \rightarrow Z\left(\mathbb{U}^{\prime}\right)
$$

such that the element $\Omega_{i}=\Omega\left(\sum_{w} k_{w\left(\omega_{i}\right)}\right)$ acts on the module $V(\lambda)$ by multiplication by the scalar $\sum_{w} q^{(2 \rho+\lambda) \cdot w\left(\omega_{i}\right)}$ for each $i, \lambda$.

To formulate the main result of this section we need an auxiliary quantum torus. Note that $\mathbb{U}_{0}$ is a Hopf algebra and that the pairing

$$
\mathbb{F}_{0} \times \mathbb{U}_{0} \rightarrow \mathcal{K}, \quad\left(q^{\mu}, k_{\lambda}\right) \mapsto q^{\mu \cdot \lambda}
$$


yields an inclusion $\mathbb{F}_{0} \subset \mathbb{U}_{0}^{*}$. Let $\mathbb{D}_{0}^{\prime}=\mathbb{F}_{0} \sharp \mathbb{U}_{0}$ be the corresponding smash product. It is a $\mathcal{K}$-algebra which is generated by elements $\partial_{\triangleright}\left(k_{\lambda}\right), \ell\left(q^{\mu}\right)$ with $\lambda, \mu \in X$. We will drop the symbols $\partial_{\triangleright}, \ell$ when there is no danger of confusion. Let $\mathbb{D}_{0} \subset \mathbb{D}_{0}^{\prime}$ be the subalgebra generated by the set $\left\{k_{\lambda}, q^{\mu} ; \lambda \in 2 X, \mu \in X\right\}$. Let $\mathbb{D}_{0, \star}^{\prime}, \mathbb{D}_{0, \star}$ be the rings of quotients with respect to the multiplicative set generated by

$$
\left\{q^{\alpha}-t^{2 j} q^{m} ; \alpha \in \Pi, m \in \mathbb{Z}, j=0,-1\right\} .
$$

There is a $\Sigma_{n}$-action on $\mathbb{D}_{0, \star}$ given by $w\left(q^{\lambda}\right)=q^{w \lambda}$ and $w\left(k_{\lambda}\right)=k_{w \lambda}$ for each $w$, $\lambda$. Let $\mathbb{D}_{0, \star}^{\Sigma_{n}}$ be the set of $\Sigma_{n}$-invariant elements. Consider the $\mathcal{K}$-algebra homomorphisms

$$
\begin{aligned}
& L^{\prime}: Z\left(\mathbb{F}^{\prime}\right) \rightarrow \mathbb{D}_{0, \star}^{\Sigma_{n}}, f \mapsto \varrho_{0}(f), \\
& L: Z\left(\mathbb{U}^{\prime}\right) \rightarrow \mathbb{D}_{0, \star}^{\Sigma_{n}}, \Omega_{i} \mapsto \sum_{w} \prod_{\alpha} \frac{t^{2} q^{w(\alpha)}-1}{q^{w(\alpha)}-1} k_{2 w\left(\omega_{i}\right)} .
\end{aligned}
$$

The product runs over all positive roots $\alpha$ such that $\alpha \cdot \omega_{i}=1$.

1.11.4. Theorem. There is a $\mathcal{K}$-algebra homomorphism $\Phi: \mathbb{T}^{0} \rightarrow \mathbb{D}_{0, \star}^{\Sigma_{n}}$ such that $z^{\prime}(f) \mapsto L^{\prime}(f)$ and $z(u) \mapsto L(u)$ for $f \in Z\left(\mathbb{F}^{\prime}\right), u \in Z\left(\mathbb{U}^{\prime}\right)$.

Proof. The proof is quite technical. To facilitate the reading we first explain the main arguments of the proof and we split it into five different steps. The homomorphism $\Phi$ comes from a quantum version of the radial part of invariant differential operators. First we prove that $\mathbb{T}^{0}$ acts on the space intertwiners of a particular $\mathbb{U}$-module $W_{t}$. This module was already introduced in [EK]. A quantum torus $\mathbb{D}_{0, \star}^{\Sigma_{n}}$ also acts on the set of generalized traces of those intertwiners. The radial part is a linear map $\phi: \mathbb{T}^{0} \rightarrow \mathbb{D}_{0, \star}^{\Sigma_{n}}$ which intertwines both actions. Our map $\Phi$ is just a renormalized version of $\phi$.

Step 1. First, we rewrite the $\mathcal{K}$-algebra $\mathbb{T}^{0}$ in a slightly different way. The linear map $\chi \otimes \varepsilon: \mathbb{U}^{\otimes 2} \rightarrow \mathcal{K}$ restricts to an algebra homomorphism $\left(\mathbb{U}^{\prime}\right)^{[2]} \rightarrow \mathcal{K}$. By 1.5.3(c) there is a $\mathcal{K}$-algebra automorphism

$$
\nu: \mathbb{E} \rightarrow \mathbb{E}, \ell\left(f \otimes f^{\prime}\right) \partial_{\triangleright}\left(v \otimes u^{\prime}\right) \mapsto \ell\left(f \otimes f^{\prime}\right) \partial_{\triangleright}\left(v^{\chi} \otimes u^{\prime}\right) .
$$

By A.7.4(e), for each $u \in \mathbb{U}^{\prime}$ we have

$$
\nu \partial_{b} \iota(u)=\partial_{b} \iota\left(u^{\chi}\right)=\partial_{b}\left((\iota u)^{\bar{\chi}}\right), \quad \nu \partial_{c}(u)=\partial_{c}\left(u^{\chi}\right) .
$$

Thus 1.5.3 $(d)$ yields a $\mathcal{K}$-algebra isomorphism

$$
\mathbb{T}^{0} \simeq \mathbb{E} / / \bar{\chi} \otimes \varepsilon\left(\iota\left(\mathbb{U}_{\pi}^{\prime}\right) \otimes \mathbb{U}^{\prime}\right) .
$$

Step 2. Next, we introduce a particular $\mathbb{U}$-module $W_{t}$ which we use to define a space of intertwiners. We define $W_{t}$ as an object of $\mathcal{M o d}\left(\mathbb{F}_{*}^{\pi}, \mathbb{U}\right)$. A $\mathcal{K}$-basis of $W_{t}$ consists of the elements $a_{\mu}, \mu \in X$. The $\mathbb{D}_{\triangleright, *}^{\pi}$-action is given by

$$
\begin{aligned}
& \partial_{\triangleright}\left(k_{\lambda}\right)\left(a_{\mu}\right)=q^{\lambda \cdot \mu} a_{\mu}, \\
& \partial_{\triangleright}\left(\dot{f}_{i}\right)\left(a_{\mu}\right)=\left(q^{\mu_{i}+1} t^{-1}-q^{-\mu_{i}-1} t\right) a_{\mu-\alpha_{i}}, \\
& \partial_{\triangleright}\left(\dot{e}_{i}\right)\left(a_{\mu}\right)=\left(q^{\mu_{i+1}+1} t^{-1}-q^{-\mu_{i+1}-1} t\right) a_{\mu+\alpha_{i}}, \\
& \ell\left(c_{1 \lambda}\right)\left(a_{\mu}\right)=q^{\tilde{\lambda} \cdot\left(\mu+\omega_{n}\right)} t^{-\tilde{\lambda} \cdot \omega_{n}} a_{\lambda+\mu} .
\end{aligned}
$$

Compare 1.7.3(c). Note that the representation of $\mathbb{U}$ on $W_{t}$ is precisely the weight $\mathbb{U}$-module denoted $W_{k}$ in [EK]. Here $k$ is a formal symbol such that $t=q^{k}$. 
Step 3. Now, we construct a $\mathbb{T}^{0}$-action on the space $I^{0}$ of intertwiners

$$
V \rightarrow W_{t} \otimes V, \quad V \in \mathcal{O}(\mathbb{U})
$$

Since the algebra $\mathbb{T}^{0}$ is a quantum reduction of the algebra $\mathbb{E}$ we will indeed define a $\iota\left(\mathbb{U}_{\pi}\right) \otimes \mathbb{U}$-equivariant $\mathbb{E}$-module $W \hat{\otimes} \mathbb{F}$ such that $I^{0}$ is the set of $\left(\iota\left(\mathbb{U}_{\pi}\right) \otimes \mathbb{U}, \bar{\chi} \otimes \varepsilon\right)$ invariant elements in $W \hat{\otimes} \mathbb{F}$. Then we apply 1.5.2(c).

The induced $\mathbb{F}_{*}$-module $W=\mathbb{F}_{*} \otimes_{\mathbb{F}_{*}^{\pi}} W_{t}$ belongs to $\operatorname{Mod}\left(\mathbb{F}_{*}, \mathbb{U}\right)$. So it is a $\mathbb{D}_{\triangleright, *}$-module. The $\mathbb{U}$-action on $W$ is given by

$$
u \triangleright(f \otimes w)=\left(u_{1} \triangleright f\right) \otimes\left(u_{2} \triangleright w\right) .
$$

Consider the $\mathbb{U}$-module $W \hat{\otimes} \mathbb{F}$. It is equipped with the action of the $\mathcal{K}$-algebra $\mathbb{D}_{\triangleright} \otimes$ $\mathbb{D}_{\triangleleft}$ such that $\mathbb{D}_{\triangleright}$ acts on $W$ as above and $\mathbb{D}_{\triangleleft}$ acts on $\mathbb{F}$ via the basic representation. In (A.7.3) we have defined a $\mathcal{K}$-algebra homomorphism

$$
\Xi: \mathbb{E} \rightarrow \mathbb{D}_{\triangleright} \otimes \mathbb{D}_{\triangleleft}, \partial_{3}\left(u \otimes v \otimes u^{\prime} \otimes v^{\prime}\right) \mapsto \partial_{\triangleleft} \iota(u) \partial_{\triangleright}(v) \otimes \partial_{\triangleleft} \iota\left(u^{\prime}\right) \partial_{\triangleright}\left(v^{\prime}\right)
$$

Thus the $\mathcal{K}$-algebra $\mathbb{E}$ acts on $W \hat{\otimes} \mathbb{F}$ via $\Xi$. The $\mathbb{U}$-action on $W \hat{\otimes} \mathbb{F}$ is given by

$$
(\operatorname{ad} u)(w \otimes f)=\left(u_{1} \triangleright w\right) \otimes\left(\operatorname{ad} u_{2}\right) f .
$$

If $u \in \mathbb{U}^{\prime}$, this action is the same as the action given by the QMM,

$$
\partial_{c}: \mathbb{U}^{\prime} \rightarrow \mathbb{E} .
$$

Now let $I=W_{t} \hat{\otimes} \mathbb{F}$. The inclusion $W_{t} \rightarrow W, w \mapsto 1 \otimes w$ is obviously $\mathbb{U}$ equivariant. Thus $I$ is indeed a $\mathbb{U}$-submodule of $W \hat{\otimes} \mathbb{F}$. The elements of $\partial_{b}\left(\iota \mathbb{U}_{\pi}^{\prime}\right)^{\bar{\chi}}$ act on $W \hat{\otimes} \mathbb{F}$ through the $\mathbb{E}$-action. They annihilate the subspace $I$. More precisely, we have

$$
I=(W \hat{\otimes} \mathbb{F})^{\pi, \chi}
$$

(left to the reader). Here we use the notation in (1.3.1). Let $I^{0} \subset I$ be the set of $\mathbb{U}$-invariant elements. We have proved the following:

- the $\mathbb{E}$-action on $W \hat{\otimes} \mathbb{F}$ factors to a $\mathbb{T}^{0}$-action on $I^{0}$ by $1.5 .2(c),(1.11 .5)$,

- the $\mathcal{K}$-vector space $I^{0}$ is spanned by the intertwining operators $V \rightarrow W_{t} \otimes V$ with $V \in \mathcal{O}(\mathbb{U})$.

Step 4. Next, we use the generalized trace on intertwiners in $I^{0}$ and we define the corresponding radial part for elements of $\mathbb{T}^{0}$. This yields a $\mathcal{K}$-algebra homomorphism $\phi$ from $\mathbb{T}^{0}$ to a quantum torus $\mathbb{D}_{0, \star}^{\prime}$. See $(1.11 .10)$ below.

Let $\hat{\mathbb{F}}_{0}$ be the $\mathcal{K}$-algebra of formal series of the form

$$
\sum_{\mu \in S} \sum_{\nu \in Y_{+}} a_{\mu \nu} \cdot \mu q^{-\nu}
$$

where $a_{\mu \nu} \in \mathcal{K}$ and $S \subset X^{\prime}$ is a finite subset. The $\mathcal{K}$-linear map

$$
\varrho=\mathrm{id} \otimes \varrho_{0}: W \hat{\otimes} \mathbb{F} \rightarrow W \otimes \hat{\mathbb{F}}_{0}
$$

factors to a linear map

$$
\varrho: I^{0} \rightarrow W_{t, 0} \otimes \hat{\mathbb{F}}_{0} .
$$

This is the generalized trace map mentioned above. Note that the zero weight subspace $W_{t, 0} \subset W_{t}$ is one-dimensional. We may abbreviate $\mathcal{K}=W_{t, 0}$, hoping it will not create any confusion. Thus we obtain a linear map

$$
\varrho: I^{0} \rightarrow \hat{\mathbb{F}}_{0} .
$$


Taking expansions yields an inclusion $\mathbb{F}_{0, \star} \subset \hat{\mathbb{F}}_{0}$. Thus the $\mathcal{K}$-algebra $\mathbb{D}_{0, \star}^{\prime}$ acts on $\hat{\mathbb{F}}_{0}$ and the $\mathcal{K}$-algebra $\mathbb{U} \otimes \mathbb{D}_{0, \star}^{\prime}$ acts on $W_{t} \otimes \hat{\mathbb{F}}_{0}$. The following is proved in [EK].

1.11.6. Lemma. (a) Fix a $\mathcal{K}$-vector space $V$. If $D \in \operatorname{Hom}\left(W_{t, 0}, V\right) \otimes \mathbb{D}_{0, \star}^{\prime}$ vanishes on $\varrho\left(I^{0}\right)$, then it is zero.

(b) There is a linear map $\nabla: \mathbb{U} \rightarrow \mathbb{U} \otimes \mathbb{D}_{0, \star}^{\prime}$ such that $(\nabla u) \varrho(g)=\varrho((z u) g)$ for each $g \in I^{0}$.

Using this lemma we can construct the radial part, which is a $\mathcal{K}$-algebra homo-

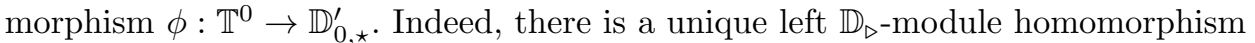

$$
\mathbb{D}_{\triangleright} \otimes \mathbb{D}_{\triangleleft} \rightarrow \mathbb{D}_{\triangleright} \otimes \mathbb{D}_{0, \star}^{\prime}, \quad \partial_{\triangleright}\left(u_{1}\right) \otimes \ell(f) \partial_{\triangleleft} \iota\left(u_{2}\right) \mapsto\left(1 \otimes \ell \varrho_{0}(f)\right)\left(\partial_{\triangleright} \otimes 1\right)(\nabla u),
$$

for each $u \in \mathbb{U}, f \in \mathbb{F}$. Composing it with $\Xi$ we get a $\mathcal{K}$-linear map

$$
\phi: \mathbb{E} \rightarrow \mathbb{D}_{\triangleright} \otimes \mathbb{D}_{0, \star}^{\prime}
$$

By 1.11.6(b) we have

$$
\phi(x) \cdot \varrho(g)=\varrho(x \cdot g), \quad \forall g \in I^{0}, x \in \mathbb{E} .
$$

In other words, the operator $\phi(x)$ is the radial part of the operator $x$.

The $\mathbb{D}_{\triangleright}$-action on $W$ yields a linear map $\mathbb{D}_{\triangleright} \rightarrow \operatorname{Hom}\left(W_{t, 0}, W\right)$. Composing it with the map $\phi$ in (1.11.7) we get a linear map

$$
\phi: \mathbb{E} \rightarrow \operatorname{Hom}\left(W_{t, 0}, W\right) \otimes \mathbb{D}_{0, \star}^{\prime} .
$$

It vanishes on the left ideal generated by $\partial_{b} \iota\left(\mathbb{U}_{\pi}^{\prime}\right)^{\bar{\chi}}$ and $\partial_{c}\left(\mathbb{U}^{\prime}\right)^{\varepsilon}$ by $1.11 .6(a)$ and (1.11.8). Thus, by (1.11.5), it factors to a linear map

$$
\mathbb{T}^{0} \rightarrow \operatorname{End}\left(W_{t, 0}\right) \otimes \mathbb{D}_{0, \star}^{\prime}=\mathbb{D}_{0, \star}^{\prime} .
$$

Let $\phi$ also denote this map. The identity (1.11.8) holds again for this new map $\phi$. Thus $\phi$ is an algebra homomorphism by 1.11.6(a).

Step 5. Finally, we renormalize the map $\phi$ in a suitable way, and we check that the new map satisfies the requirements in the theorem.

If $f \in Z\left(\mathbb{F}^{\prime}\right)$, then we have $\Xi \gamma^{\prime} \ell(f)=1 \otimes \ell(f)$ by A.7.4(d). Thus $\phi z^{\prime}(f)=\ell \varrho_{0}(f)$. We also have $\phi z(u)=\nabla(u)$ for each $u \in Z\left(\mathbb{U}^{\prime}\right)$. Let $\pi \in \hat{\mathbb{F}}_{0}$ be the expansion of the infinite product

$$
q^{-\rho} t^{\rho} \prod_{i, \alpha}\left(1-q^{2 i} q^{-\alpha}\right) /\left(1-q^{2 i-2} t^{2} q^{-\alpha}\right) .
$$

Here $i$ runs over all positive integers and $\alpha$ over all positive roots. Let $\Phi$ be the composition of $\phi$ and the conjugation by $\ell(\pi)^{-1}$. The map $\Phi$ is an algebra homomorphism such that $\Phi(f)=L^{\prime}(f)$ for $f \in Z\left(\mathbb{F}^{\prime}\right)$. By [EK, thm. 4] we also have $\Phi(u)=L(u)$ for $u \in Z\left(\mathbb{U}^{\prime}\right)$.

Now, we must check that $\Phi\left(\mathbb{T}^{0}\right) \subset \mathbb{D}_{0, \star}^{\Sigma_{n}}$. The inclusion $\Phi\left(\mathbb{T}^{0}\right) \subset \mathbb{D}_{0, \star}$ is a routine computation using the inductive construction of $\nabla$ in [op. cit]. Let us concentrate on the $\Sigma_{n}$-invariance. Fix an element $x \in \mathbb{T}^{0}$. We know that $\Phi(x) \in \mathbb{D}_{0, \star}$. We must prove that it is $\Sigma_{n}$-invariant. To do this we will use the following refinement of 1.11.6. For $\lambda \in X$ there is an unique nonzero intertwiner

$$
g_{\lambda}: M\left(q^{\lambda} t^{\rho}\right) \rightarrow W_{t} \otimes M\left(q^{\lambda} t^{\rho}\right),
$$

up to a multiplicative scalar, because $M\left(q^{\lambda} t^{\rho}\right)$ is an irreducible Verma module. 
1.11.11. Lemma. (a) If $\lambda+\rho \in X_{+}$, then $\pi^{-1} \varrho\left(g_{\lambda}\right) \in \mathbb{F}_{0}^{\Sigma_{n}}$.

(b) Fix $D \in \mathbb{D}_{0, \star}^{\prime}$. If $D \varrho\left(g_{\lambda}\right)=0$ for all weight $\lambda$ which is far enough into the dominant Weyl chamber, then $D=0$.

(c) For each $x \in \mathbb{T}^{0}$ there is a finite subset $\Lambda^{\prime} \subset X_{+}$such that $x \cdot g_{\lambda}$ belongs to $\sum_{\lambda^{\prime} \in \Lambda^{\prime}} I\left(W_{t}, V\left(\lambda^{\prime}\right) \otimes M\left(q^{\lambda} t^{\rho}\right)\right)$ for all weight $\lambda$.

(d) For each $\lambda^{\prime} \in X$ the module $V\left(\lambda^{\prime}\right) \otimes M\left(q^{\lambda} t^{\rho}\right)$ admits a flag whose quotients are isomorphic to the Verma modules $M\left(q^{\mu} t^{\rho}\right)$ such that $\mu-\lambda$ belongs to the multiset of weights of $V\left(\lambda^{\prime}\right)$, counted with their multiplicities.

By $1.11 .11(c),(d)$ there is a finite subset $\Lambda^{\prime} \subset X$ such that $x \cdot g_{\lambda}$ is a linear combination of the intertwiners $g_{\mu}$ with $\mu \in \lambda+\Lambda^{\prime}$ for each $\lambda \in X$. If $\lambda$ is far enough into the dominant Weyl chamber, then we have $\lambda+\rho+\Lambda^{\prime} \in X_{+}$. So 1.11.8 and 1.11.11(a) imply that

$$
(\Phi(x)-w \Phi(x)) \cdot \pi^{-1} \varrho\left(g_{\lambda}\right)=0, \quad \forall w \in \Sigma_{n} .
$$

Therefore, $\Phi(x)$ is $\Sigma_{n}$-invariant by $1.11 .11(b)$.

Proof of 1.11.11. (a) and $(b)$ are proved in [EK], $(c)$ is left to the reader and $(d)$ is well known.

\section{Roots OF Unity}

2.1. A reminder of Poisson geometry. Let A be any CNID. By a Poisson A-algebra we mean a commutative affine A-algebra with a Lie bracket satisfying the Leibniz rule. A Poisson A-scheme is an A-scheme $X$ such that $\mathcal{O}(U)$ is a Poisson A-algebra for each open set $U \subset X$. A Poisson group is a Poisson group $\alpha$-scheme whose structural morphisms are Poisson homomorphisms. In the same way we define a Poisson action of a Poisson group on a Poisson scheme. See [L] for backround on Poisson geometry.

Let $D=G^{2}$ and let $G \subset D$ denote the diagonal subgroup. Consider the groups

$$
G^{\vee}=\left\{g^{\vee}=\left(u_{-}^{-1} h^{-1}, u_{+} h\right) \in D ; u_{ \pm} \in U_{ \pm}, h \in H\right\}, \quad D^{\vee}=G \times G^{\vee} .
$$

We use the following notation in $\mathfrak{g}$ :

$$
\bar{e}_{i}=e_{i, i+1}, \bar{f}_{i}=e_{i+1, i}, \bar{h}_{\lambda}=\sum_{i} \lambda_{i} e_{i i}, \forall i \in I, \lambda \in X .
$$

We equip $\mathfrak{g}$ with the nondegenerate invariant bilinear form $\langle:\rangle$ such that

$$
\left\langle\bar{h}_{\lambda}: \bar{h}_{\lambda^{\prime}}\right\rangle=\lambda \cdot \lambda^{\prime}, \quad \lambda, \lambda^{\prime} \in X
$$

Let $\mathfrak{d}=\mathfrak{g} \times \mathfrak{g}$ be the Lie algebra of $D$. It is equipped with the nondegenerate invariant bilinear form given by

$$
\left\langle\left(x, x^{\prime}\right):\left(y, y^{\prime}\right)\right\rangle=-\langle x: y\rangle+\left\langle x^{\prime}: y^{\prime}\right\rangle .
$$

This yields a linear isomorphism

$$
\sharp: \mathfrak{d}^{*} \rightarrow \mathfrak{d} \text {. }
$$

It factors to an isomorphism $\sharp:\left(\mathfrak{g}^{\vee}\right)^{*} \rightarrow \mathfrak{g}$. Let $\sharp$ also denote the inverse map $\mathfrak{g} \rightarrow\left(\mathfrak{g}^{\vee}\right)^{*}$. From now on it will be simpler to use the following notation:

$$
\mathfrak{g}^{*}=\mathfrak{g}^{\vee}, \quad G^{*}=G^{\vee}, \quad D^{*}=D^{\vee} .
$$

Equip $G$ with the Drinfeld-Sklyanin bracket and $G^{*}$ with the dual bracket. Let $X$ be a Poisson scheme with a right Poisson $G$-action. The infinitesimal action 
of $x \in \mathfrak{g}$ on $X$ is a derivation $x \triangleright$ of $\mathcal{O}_{X}$. For $f \in \mathcal{O}\left(G^{*}\right)$ we abbreviate $f^{\sharp}=$ $\sharp\left(\mathrm{d}_{e} f\right)$, an element in $\mathfrak{g}$. A $G$-equivariant Poisson moment map is a Poisson algebra homomorphism $\partial: \mathcal{O}\left(G^{*}\right) \rightarrow \mathcal{O}(X)$ such that

$$
\{\partial f, \varphi\}=\left(f_{1}^{\sharp} \triangleright \varphi\right) \partial f_{2}, \quad \forall f, \varphi .
$$

Here the map $f \mapsto f_{1} \otimes f_{2}$ is the comultiplication of $\mathcal{O}\left(G^{*}\right)$. Let $U \subset X$ be a $G$ invariant open subset. It is well known that $\mathcal{O}(U)^{G} \subset \mathcal{O}(U)$ is a Poisson subalgebra. Let $I \subset \mathcal{O}(U)$ be the ideal generated by $\partial \mathcal{O}\left(G^{*}\right)^{\varepsilon}$. The Poisson bracket on $\mathcal{O}(U)$ yields a Poisson bracket on the algebra $\mathrm{B}=(\mathcal{O}(U) / I)^{G}$ such that the obvious map $\mathcal{O}(U)^{G} \rightarrow$ B is a Poisson algebra homomorphism.

We define Poisson brackets on the groups $D, D^{*}$ as follows. Let $\mathfrak{d}$ be the Lie algebra of $D$. We define a bivector $\pi_{0} \in \wedge^{2} \mathfrak{d}$ by the formula

$$
\left\langle\pi_{0}: x \wedge y\right\rangle=\left\langle x-x^{*}: y\right\rangle-\left\langle x^{*}: y\right\rangle .
$$

Here $x^{*} \in \mathfrak{g}^{*}$, the Lie algebra of $G^{*}$, and $x-x^{*} \in \mathfrak{g}$, the diagonal of $\mathfrak{d}$. The bivector field on $D$ is given by $\pi_{D}(d)=d \triangleright \pi_{0}+\pi_{0} \triangleleft d$ where $\triangleright, \triangleleft$ are the left and right translations. The multiplication in $D$ gives the étale map

$$
D^{*} \rightarrow D, \quad\left(g, g^{*}\right) \mapsto(g, g) g^{*} .
$$

Thus the Poisson bracket on $D$ lifts to $D^{*}$. Let $\pi_{G}, \pi_{G^{*}}, \pi_{D^{*}}$ be the Poisson bivector fields of $G, G^{*}, D^{*}$. An explicit formula for $\pi_{D^{*}}$ is given in A.8.6(a) below. Recall that $G_{\Sigma} \subset G$ is the open Bruhat cell. We set

$$
D_{\Sigma}=\left\{(g, h) \in D ; h \in G_{\Sigma}, g h g^{-1} \in G_{\Sigma}\right\} .
$$

2.2. QDO on $G$ at roots of unity. Now we collect several facts on quantum groups at roots of unity. The reader may skip this section and return when needed.

Recall that $\mathcal{K}$ is an extension of $\mathbb{Q}(q)$. Let $\mathcal{A} \subset \mathcal{K}$ be a subring such that $\mathcal{K}=\operatorname{Frac}(\mathcal{A})$. We will assume that $\mathbb{Z}\left[q, q^{-1}\right] \subset \mathcal{A}$. Fix a unit $t \in \mathcal{A}$. Let $\mathcal{A} \rightarrow \mathrm{A}$ be a ring homomorphism. Let $\tau, \zeta \in \mathrm{A}$ be the images of $q, t$. Let $l$ be the order of $\tau$ in the multiplicative group of A. We are mainly interested in the case where $l$ is finite. In this case we will always assume that $l=p^{e}$, an odd prime power with $e>0$. Occasionally we may use an A-algebra $\mathrm{k}$. We will always assume that $\mathcal{A}$, $\mathrm{A}, \mathrm{k}$ are CNID with global dimension $\leqslant 2$. We set $\mathrm{k}=$ Frac $(\mathrm{A})$. Unless specified otherwise we will also assume that

$$
\mathcal{A} \rightarrow \mathrm{A} \rightarrow \mathrm{k}
$$

is a diagram of local rings and that the residual characteristic is zero or is large enough. We call $(\mathcal{A}, \mathrm{A}, \mathrm{k})$ a modular triple and $(\mathcal{A}, \mathrm{A}),(\mathrm{A}, \mathrm{k})$ modular pairs. Examples of modular triples are given in 2.2.5 below.

For any $\mathcal{A}$-module $\mathbb{V}_{\mathcal{A}}$ we abbreviate $\mathbb{V}_{\mathrm{A}}=\mathbb{V}_{\mathcal{A}} \otimes \mathrm{A}$. If $l$ is finite we write $\mathbf{V}_{\mathrm{A}}=\mathbb{V}_{\mathrm{A}}$. If the ring $\mathrm{A}$ is clear from the context we drop it.

We use the following notation for $q$-numbers: $[r]=\left(q^{r}-q^{-r}\right) /\left(q-q^{-1}\right)$ and $[r] !=\prod_{s=1}^{r}[s]$ !. For each $x \in \mathbb{U}$ we call $x^{(r)}=x^{r} /[r]$ ! the quantum divided power.

Let $\dot{U}_{\mathcal{A}} \subset \mathbb{U}$ be the Lusztig lattice, as defined in [DL, sec. 3.4]. See also BG3, sec. III.7] for a review. It is the $\mathcal{A}$-subalgebra generated by $e_{\alpha}^{(r)}, f_{\alpha}^{(r)}, k_{\lambda}$ and $h_{\lambda}^{(r)}=\prod_{s=1}^{r}\left(k_{\lambda} q^{1-s}-1\right)\left(q^{s}-1\right)^{-1}$ where $\alpha \in S, r \in \mathbb{Z}_{+}$and $\lambda \in X$.

Let $\mathbb{U}_{\mathcal{A}} \subset \mathbb{U}$ be the Deconcini-Kac-Procesi lattice used in [DP, sec. 12.1]. See also BG3, sec. III.6] for a review. It is the $\mathcal{A}$-subalgebra generated by the elements $\dot{e}_{\alpha}, \dot{f}_{\alpha}$ and $k_{\lambda}$. Here $\alpha$ is a simple root and $\lambda \in X$. 
Let $\dot{\mathbb{U}}_{0, \mathcal{A}}, \mathbb{U}_{0, \mathcal{A}}$ be the corresponding Cartan subalgebras. Recall that we have fixed a reduced expression for $w_{0}$. This yields an ordering $\beta_{1} \leqslant \beta_{2} \leqslant \cdots \leqslant \beta_{N}$ of the positive roots. For any sequence $m=\left(m_{i}\right)$ of integers $\geqslant 0$ we set

$$
|m|=\sum_{i} m_{i} \beta_{i}
$$

Given two sequences $m=\left(m_{i}\right), n=\left(n_{i}\right)$ as above we define the monomials

$$
\dot{e}^{m} k_{\lambda} \iota\left(\dot{f}^{n}\right)=\dot{e}_{\beta_{1}}^{m_{1}} \ldots \dot{e}_{\beta_{N}}^{m_{N}} k_{\lambda} \dot{f}_{\beta_{N}}^{n_{N}} \ldots \dot{f}_{\beta_{1}}^{n_{1}} .
$$

These monomials form an $\mathcal{A}$-basis of $\mathbb{U}_{\mathcal{A}}$.

Let $\mathbb{F}_{\mathcal{A}} \subset \mathbb{F}$ be the Hopf $\mathcal{A}$-algebra dual to $\dot{\mathbb{U}}_{\mathcal{A}}$, i.e., the sum of the coefficient spaces $\operatorname{cf}\left(V_{\mathcal{A}}(\lambda)\right), \lambda \in X_{+}$, of the $\dot{U}_{\mathcal{A}}$-modules of type 1 which are free of finite rank as $\mathcal{A}$-modules. It is known that $\mathbb{F}_{\mathcal{A}}$ is a free $\mathcal{A}$-module.

Set $\mathbb{F}^{\prime}{ }_{\mathcal{A}}=\mathbb{F}_{\mathcal{A}}$ as an $\mathcal{A}$-module with the multiplication $m^{\prime}$ from section 1.7. Also, write $\dot{\mathbb{H}}_{\mathcal{A}}=\dot{\mathbb{U}}_{\mathcal{A}}^{\otimes 4}$ as an $\mathcal{A}$-algebra with the coproduct from section 1.10.

The other lattices are defined in a similar way. For instance, $\mathbb{G}_{\mathcal{A}}=\mathbb{F}_{\mathcal{A}} \otimes \mathbb{F}_{\mathcal{A}}$ as an $\mathcal{A}$-module with the multiplication in section 1.10, $\mathbb{U}_{\mathcal{A}}^{[2]}=\mathbb{U}_{\mathcal{A}}^{\otimes 2}$ as an $\mathcal{A}$ algebra with the coproduct in section 1.7 (up to some completion), $\mathbb{U}_{\mathcal{A}}^{\prime}=\kappa\left(\mathbb{F}_{\mathcal{A}}^{\prime}\right)$, $\left(\mathbb{U}_{\mathcal{A}}^{\prime}\right)^{e}=\iota\left(\mathbb{U}_{\mathcal{A}}^{\prime}\right) \otimes \mathbb{U}_{\mathcal{A}}^{\prime},\left(\mathbb{U}_{\mathcal{A}}^{\prime}\right)^{[2]}=\varpi_{2}\left(\mathbb{U}_{\mathcal{A}}^{\prime} \otimes \mathbb{U}_{\mathcal{A}}^{\prime}\right), \mathbb{D}_{\mathcal{A}}^{\prime}=\mathbb{F}_{\mathcal{A}}^{\prime} \sharp \mathbb{U}_{\mathcal{A}}^{\prime}, \mathbb{D}_{\triangleright, \mathcal{A}}^{\prime}=\mathbb{F}_{\mathcal{A}}^{\prime} \sharp \mathbb{U}_{\mathcal{A}}$,

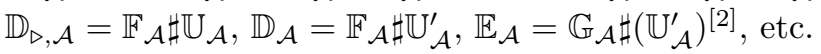

Now let us assume that $l$ is finite. Let $\mathfrak{U}$ be the hyperalgebra of $G$, an Aalgebra. Let $\mathbf{u} \subset \dot{\mathbf{U}}$ be the image of the canonical map $\mathbf{U} \rightarrow \dot{\mathbf{U}}$, a normal Hopf subalgebra of $\dot{\mathbf{U}}$. See [L1, sec. 5.3] for a proof of the normality of u. Lusztig's Frobenius is a ring homomorphism $\phi: \dot{\mathbf{U}} \rightarrow \mathfrak{U}$, see [L2, sec. 35], [DL, thm. 6.3]. The kernel of $\boldsymbol{\phi}$ is generated by the augmentation ideal of $\mathbf{u}$. Therefore, given a module $V \in \mathcal{M} \boldsymbol{o d}^{\mathrm{lf}}(\dot{\mathbf{U}})$ such that $\mathbf{u}$ acts trivially on $V$, the representation of $\dot{\mathbf{U}}$ on $V$ factors through $\phi$, yielding a representation of $\mathfrak{U}$. Since $V$ is locally finite, this is indeed a representation of $G$. We define $\dot{\mathbf{U}}_{\pi}, \mathbf{u}_{\pi}$ in the same way. Note that our definition of $\mathbf{u}$ differs slightly from Lusztig's restricted quantized enveloping algebra. Indeed Lusztig's algebra has rank $2^{n} l^{n^{2}}$ while $\mathbf{u}$ has rank $l^{n^{2}}$.

Let $\mathcal{U} \subset Z(\mathbf{U})$ be the A-subalgebra generated by the elements

$$
z_{\lambda}=k_{\lambda}^{l}, \quad y_{\alpha}=\iota\left(\dot{f}_{\alpha}^{l}\right), \quad x_{\alpha}=\dot{e}_{\alpha}^{l}, \quad \forall \alpha \in \Pi_{+}, \forall \lambda \in X .
$$

We abbreviate $x_{i}=x_{\alpha_{i}}, y_{i}=y_{\alpha_{i}}$ and $z_{i}=z_{\alpha_{i}}$ for all $i \in I$. Let $\mathcal{F} \subset Z(\mathbf{F})$ be the A-subalgebra generated by the elements

$$
\left(c_{i j}\right)^{l}, \quad i, j=1,2, \ldots, n .
$$

It is known that $\mathcal{U}, \mathcal{F}$ are Hopf subalgebras of $\mathbf{U}, \mathbf{F}$. They are also direct summands as $\alpha$-modules. We set

$$
\mathcal{U}^{\prime}=\kappa(\mathcal{F}), \quad \mathcal{D}=\ell(\mathcal{F}) \partial_{\triangleright}\left(\mathcal{U}^{\prime}\right), \quad \mathcal{D}_{\triangleright}=\ell(\mathcal{F}) \partial_{\triangleright}(\mathcal{U}) .
$$

We define the following maps:

$$
\begin{aligned}
& \mathfrak{s}_{0}: G^{*} \rightarrow G, g^{*} \mapsto u_{+} h^{2} u_{-}, \\
& \mathfrak{a}: G \rightarrow G, g \mapsto^{\mathrm{T}} g^{-1} .
\end{aligned}
$$

Here the superscript ${ }^{\mathrm{T}}$ holds for the transpose matrix. Note that $\mathfrak{s}_{0}$ is an étale cover of $\mathfrak{s}_{0}(G)=G_{\Sigma}$ and that $\mathfrak{a}$ is an involution. Consider the composed homomorphism

$$
\mathfrak{s}=\mathfrak{a} \circ \mathfrak{s}_{0}: G^{*} \rightarrow G .
$$


Setting $\hbar=l\left(q^{l}-q^{-l}\right), l\left(q^{l}-1\right)$ respectively in A.10.2 we define Poisson brackets on $\mathcal{U}, \mathcal{F}$. An explicit computation shows that they are defined over $\alpha$. The following is proved in section A.8.

2.2.3. Proposition. (a) The A-algebras $\mathbb{U}_{\mathrm{A}}, \mathbb{F}_{\mathrm{A}}, \mathbb{U}_{\mathrm{A}}^{\prime}, \mathbb{F}_{\mathrm{A}}^{\prime}$ and $\mathbb{D}_{\triangleright, \mathrm{A}}$ are NID. The map $\kappa: \mathbb{F}_{\mathrm{A}}^{\prime} \rightarrow \mathbb{U}_{\mathrm{A}}^{\prime}$ is an A-algebra isomorphism. We have $\mathbb{U}_{\mathrm{A}}^{\prime} \subset \mathbb{U}_{\mathrm{A}}$. Further, $\mathbb{F}_{\mathrm{A}}, \mathbb{D}_{\mathrm{A}}$ are $\mathbb{U}_{\mathrm{A}}^{e}$-algebras and $\mathbb{U}_{\mathrm{A}}^{\prime}$ is an $($ ad比 $)$-algebra. We have $\partial_{\triangleright}\left(\mathbb{U}_{\mathrm{A}}^{\prime}\right), \partial_{\triangleleft}\left(\mathbb{U}_{\mathrm{A}}^{\prime}\right)$, $\partial_{2}\left(\left(\mathbb{U}_{\mathrm{A}}^{\prime}\right)^{[2]}\right) \subset \mathbb{D}_{\mathrm{A}}$ and $\psi\left(\mathbb{D}_{\mathrm{A}} \otimes \mathbb{D}_{\mathrm{A}}^{\prime}\right), \partial_{3}\left(\mathbb{H}_{\mathrm{A}}^{\prime}\right) \subset \mathbb{E}_{\mathrm{A}}$.

(b) The map $\kappa$ factors to an algebra isomorphism $\mathcal{F} \rightarrow \mathcal{U}^{\prime}$. The maps $\partial_{\triangleright}$, $\partial_{\triangleleft}$ factor to algebra homomorphisms $\mathcal{U}^{\prime} \rightarrow \mathcal{D}$. Further, $\mathcal{U}^{\prime}, \mathcal{U}$ are $(\operatorname{ad} G)$ algebras, $\mathcal{F}, \mathcal{D}$ are $G^{2}$-algebras and $\kappa: \mathcal{F} \rightarrow \mathcal{U}^{\prime}$ commutes with the adjoint G-action.

(c) There are Poisson-Hopf algebra isomorphisms

$$
\mathfrak{z}_{U}: \mathcal{O}\left(G^{*}\right) \rightarrow \mathcal{U}, \quad \mathfrak{z}_{F}: \mathcal{O}(G) \rightarrow \mathcal{F} .
$$

The map $\mathfrak{z}_{F}$ is $G^{2}$-equivariant. The algebra homomorphism

$$
\mathfrak{z}^{\prime}=\kappa \circ \mathfrak{z}_{F}: \mathcal{O}(G) \rightarrow \mathcal{U}^{\prime}
$$

is $(\operatorname{ad} G)$-equivariant and $\mathfrak{z} U^{\prime}=\mathfrak{z} U \circ \mathfrak{s}^{*}$. There is a $G^{2}$-algebra isomorphism

$$
\mathfrak{z} D: \mathcal{O}(D) \rightarrow \mathcal{D} \text {. }
$$

(d) We have that $\mathbf{F}$ is a free $\mathcal{F}$-module of rank $l^{n^{2}}, \mathbf{u}$ is a free A-module of rank $l^{n^{2}}, \mathbf{U}$ is a free $\mathcal{U}$-module of rank $l^{n^{2}}$ and $\mathbf{U}^{\prime}$ is a free $\mathcal{U}^{\prime}$-module of rank $l^{n^{2}}$. Further, $\mathcal{D} \subset Z(\mathbf{D}), Z\left(\mathbf{D}^{\prime}\right)$ and $\mathbf{D}, \mathbf{D}^{\prime}$ are free $\mathcal{D}$-modules of rank $l^{2 n^{2}}$. Finally, $\mathcal{F} \subset Z\left(\mathbf{F}^{\prime}\right)$ and $\mathbf{F}^{\prime}$ is a free $\mathcal{F}$-module of rank $l^{n^{2}}$.

(e) The $\dot{\mathbf{U}}^{e}$-action on $\mathbf{F}, \mathbf{D}$ preserves the subalgebras $\mathcal{F}, \mathcal{D}$. The adjoint $\dot{\mathbf{U}}$ action on $\mathbf{F}^{\prime}, \mathbf{D}^{\prime}$ preserves the subalgebras $\mathcal{F}, \mathcal{D}$.

(f) Assume that $\mathrm{A}=\mathbb{C}$. We have $\mathcal{D} \simeq Z(\mathbf{D}), Z\left(\mathbf{D}^{\prime}\right)$ and $\mathcal{D}_{\triangleright} \simeq Z\left(\mathbf{D}_{\triangleright}\right)$. There is a Poisson algebra isomorphism $\mathcal{O}\left(D^{*}\right) \rightarrow \mathcal{D}_{\triangleright}$. The algebra $\mathbf{D}_{\triangleright}$ is a maximal order of PI-degree $l^{n^{2}}$ and a Poisson $\mathcal{O}\left(D^{*}\right)$-order.

Note that $\mathbf{D}, \mathbf{D}^{\prime}$ are not Azumaya algebras, but we have precise information on the Azumaya locus. Let $\mathbf{D}_{\Sigma}$ be the localization of $\mathbf{D}$ at $\partial_{\triangleright}(\Sigma) \cup \partial_{\triangleleft}(\Sigma)$ and $\mathbf{D}_{\Sigma}^{\prime}$ the localization of $\mathbf{D}^{\prime}$ at $\partial_{2} \varpi_{2}(\Sigma \otimes \Sigma)$. The following is proved in section A.8.

2.2.4. Corollary. If $\mathrm{A}=\mathbb{C}$, then $\mathbf{D}_{\Sigma}, \mathbf{D}_{\Sigma}^{\prime}$ are Azumaya algebras over $D_{\Sigma}$ of PI-degree $l^{n^{2}}$.

We make special use of the modular triples $\left(\mathcal{A}_{b}, \mathrm{~A}_{b}, \mathrm{k}_{b}\right),\left(\mathcal{A}_{c}, \mathrm{~A}_{c}, \mathrm{k}_{c}\right)$ below. We indicate an $\mathrm{A}_{b}$-module by a subscript $b$ and an $\mathrm{A}_{c}$-module by a subscript $c$.

\subsubsection{Examples.}

(a) Given $\tau, \zeta \in \mathbb{C}^{\times}$we put $\mathrm{k}_{b}=\mathbb{C}\left[q^{ \pm 1}, t^{ \pm 1}\right] /(q-\tau, t-\zeta)$. Let $\mathcal{A}_{b}, \mathrm{~A}_{b}$ be the local ring of $\mathbb{C}\left[q^{ \pm 1}, t^{ \pm 1}\right], \mathbb{C}\left[q^{ \pm 1}, t^{ \pm 1}\right] /(q-\tau)$ at $\mathrm{k}_{b}$. So $\mathrm{A}_{b}$ is a DVR. We write $\mathrm{k}_{b}=\operatorname{Frac}\left(\mathrm{A}_{b}\right)$. We abbreviate $\mathrm{k}_{b}=\mathbb{C}$, hoping it will not create any confusion.

(b) Fix $c=k / m \in \mathbb{Q}^{\times}$with $(m, k)=1$. Let $\Gamma_{c}=\operatorname{Spec}\left(\mathbb{Z}\left[q^{ \pm 1}, t^{ \pm 1}\right] /\left(q^{k}-t^{m}\right)\right)$. Fix $(\tau, \zeta) \in \Gamma_{c}(\mathbb{C})$ with $\tau$ a root of unity of order $l=p^{e}$. We define $\mathrm{A}_{c} \subset \mathbb{C}$ as the localization of the subring of $\mathbb{C}$ generated by $\tau, \zeta$ at a finite field $\mathrm{k}_{c}$ of characteristic $p$ such that $\tau$ maps to 1 in $\mathrm{k}_{c}$. So $\mathrm{A}_{c}$ is a DVR. Let $\mathcal{A}_{c}$ be the local ring of $\Gamma_{c}$ at $\mathrm{k}_{c}$ and $\mathrm{k}_{c}=\operatorname{Frac}\left(\mathrm{A}_{c}\right)$. See section A.8 for details. 


\subsubsection{Remarks.}

(a) If $\tau=1$, then we have $\mathbf{U}=\mathcal{O}\left(G^{*}\right), \mathbf{F}=\mathcal{O}(G)$ and $\mathbf{U}^{\prime}=\mathcal{O}(G)$. See DP, sec. 12.1] for the first isomorphism.

(b) The map $\psi$ in 1.10.3(a) yields linear isomorphisms $\mathbb{R}_{\mathrm{A}} \otimes \mathbb{D}_{\mathrm{A}}^{\prime} \rightarrow \mathbb{S}_{\mathrm{A}}$ and $\mathbb{R}_{\mathrm{A}}^{\pi} \otimes \mathbb{D}_{\mathrm{A}}^{\prime} \rightarrow \mathbb{S}_{\mathrm{A}}^{\pi}$.

(c) The $G^{2}$-action on $D$ in 2.2.3(c) and (1.8.1) is given by

$$
\left(h, h^{\prime}\right) \triangleright\left(g, g^{\prime}\right)=\left(h g\left(h^{\prime}\right)^{-1}, h g^{\prime} h^{-1}\right) .
$$

(d) Let $\chi, \bar{\chi}$ be as in (1.11.1). The A-algebra $\left(\mathbb{U}_{\mathrm{A}}^{\prime}\right)^{\bar{\chi}}$ is generated by $\left\{\kappa\left(c_{i j}\right)-\right.$ $\left.q^{-2} t^{2} \delta_{i j}\right\}$. Thus $\left(\mathcal{U}^{\prime}\right)^{\bar{\chi}}$ is generated by $\left\{\mathfrak{z}_{U^{\prime}}\left(\bar{c}_{i j}\right)-\zeta^{2 l} \delta_{i j}\right\}$.

(e) The A-algebras $\mathbb{R}_{\mathrm{A}}^{\pi}, \mathbb{S}_{\mathrm{A}}^{\pi}$ are ID. Compare 1.9.1(b) and 1.10.3(b).

$(f)$ The subscripts $i, *$ indicate quotient rings as before. The A-modules $\mathbb{F}_{\mathrm{A}, i}$, $\mathbb{D}_{\mathrm{A}, i}, \mathbb{E}_{\mathrm{A}, i}$ are flat. Indeed, it is enough to prove that $\mathbb{F}_{\mathcal{A}, i}$ is $\mathcal{A}$-flat for $\mathcal{A}=\mathbb{Z}\left[q^{ \pm 1}\right]$, and this follows from the fact that $\mathbb{F}_{\mathcal{A}}$ is $\mathcal{A}$-flat and $\mathbb{F}_{\mathcal{A}, i}$ is $\mathbb{F}_{\mathcal{A}}$-flat; see [MR, prop. 2.1.16(ii)].

2.3. Lattices and invariants. In this section we collect some facts on invariants. The reader may skip this section and return when needed. Fix a modular triple $(\mathcal{A}, \mathrm{A}, \mathrm{k})$. For each $m \in \mathbb{Z}$ there is an unique $\mathcal{K}$-algebra homomorphism

$$
\langle m\rangle: \mathbb{U} \rightarrow \mathcal{K}, k_{\lambda} \mapsto q^{-m \omega_{n} \cdot \lambda} .
$$

It maps $\dot{\mathbb{U}}_{\mathcal{A}}$ to $\mathcal{A}$. Thus it yields an A-algebra homomorphism

$$
\langle m\rangle: \dot{\mathbb{U}}_{\mathrm{A}} \rightarrow \mathrm{A}
$$

Given $V \in \boldsymbol{M} \boldsymbol{M} \boldsymbol{d}\left(\dot{\mathbb{U}}_{\mathrm{A}}\right)$ we define a new $\dot{\mathbb{U}}_{\mathrm{A}}$-module $V\langle m\rangle$ by tensoring $V$ with $\langle m\rangle$. Consider the following space of semi-invariant elements:

$$
V^{+}=\bigoplus_{m \geqslant 0} V^{m}, \quad V^{m}=(V\langle m\rangle)^{\dot{\mathbb{U}}_{\mathrm{A}}} .
$$

If $V$ is a $\dot{U}_{\mathrm{A}}$-algebra, then $V^{+}$is a $\mathbb{Z}_{+}$-graded A-algebra.

Let $\left(\mathbb{U}_{\pi, \mathrm{A}}^{\prime}\right)^{\varepsilon} \subset \mathbb{U}_{\mathrm{A}}^{\prime}$ be the A-subalgebra generated by $\left\{\kappa \iota\left(c_{i j}\right)-\delta_{i j} ; j \neq 1\right\}$ and let $\mathbb{I}_{V, \mathrm{~A}} \subset \mathbb{U}_{\mathrm{A}}^{\prime}$ be the right ideal generated by $\left(\mathbb{U}_{\pi, \mathrm{A}}^{\prime}\right)^{\varepsilon}$. Let $\mathbb{I}_{R, \mathrm{~A}} \subset \mathbb{D}_{\mathrm{A}}, \mathbb{I}_{S, \mathrm{~A}} \subset \mathbb{E}_{\mathrm{A}}$ be the left ideals generated by $\partial_{\triangleleft}\left(\mathbb{U}_{\pi, \mathrm{A}}^{\prime}\right)^{\varepsilon}, \partial_{b} \iota\left(\mathbb{U}_{\pi, \mathrm{A}}^{\prime}\right)^{\varepsilon}$, respectively. We have free $\alpha$-modules

$$
\mathbb{V}_{\mathrm{A}}=\mathbb{U}_{\mathrm{A}}^{\prime} / \mathbb{I}_{V, \mathrm{~A}}, \quad \mathbb{R}_{\mathrm{A}}=\mathbb{D}_{\mathrm{A}} / \mathbb{I}_{R, \mathrm{~A}}, \quad \mathbb{S}_{\mathrm{A}}=\mathbb{E}_{\mathrm{A}} / \mathbb{I}_{S, \mathrm{~A}}
$$

Let ${ }^{\pi} \mathbb{F}_{\mathrm{A}}, \mathbb{F}_{\mathrm{A}}^{\pi} \subset \mathbb{F}_{\mathrm{A}}$ be the A-subalgebras generated by the sets $\left\{c_{i 1}\right\},\left\{c_{1 i}\right\}$. Note that $\mathbb{R}_{\mathrm{A}}$ is the localization of $\mathbb{R}_{+, \mathrm{A}}=\mathbb{F}_{\mathrm{A}} \otimes \mathbb{F}_{\mathrm{A}}$ at $1 \otimes c_{11}$; see 1.7.2(b).

For any $\dot{\mathbb{U}}_{\pi, \mathrm{A}}$-module $V$ we abbreviate $V^{\pi}=V^{\dot{\mathbb{U}}_{\pi, \mathrm{A}} \text {. Let }}$

$$
\mathbb{D}_{\mathrm{A}}^{\pi}=\left(\mathbb{D}_{\mathrm{A}}\right)^{\pi}, \quad \mathbb{R}_{\mathrm{A}}^{\pi}=\left(\mathbb{R}_{\mathrm{A}}\right)^{\pi}, \quad \mathbb{S}_{\mathrm{A}}^{\pi}=\left(\mathbb{S}_{\mathrm{A}}\right)^{\pi} .
$$

By 1.5.2 there are A-algebra structures on $\mathbb{D}_{\mathrm{A}}^{\pi}, \mathbb{R}_{\mathrm{A}}^{\pi}$ and $\mathbb{S}_{\mathrm{A}}^{\pi}$. Compare these with the $\mathcal{K}$-algebra structures on $\mathbb{D}^{\pi}, \mathbb{R}^{\pi}$ and $\mathbb{S}^{\pi}$ in sections $1.9,1.10$.

Recall that an element $a \in \mathrm{A}$ acts regularly on an A-module $V$ if its annihilator in $V$ is $\{0\}$. The following are proved in section A.11 using good filtrations.

2.3.3. Lemma. (a) Let $V \in \operatorname{Mod}^{l f}\left(\dot{U}_{\mathrm{A}}\right)$. If $V$ is a flat $\mathrm{A}$-module, then $V^{+}$is again a flat $\mathrm{A}$-module. If $\mathrm{k}=\mathrm{A} / a \mathrm{~A}$ and a acts regularly on $V$, then we have

$$
V^{+} \otimes \mathrm{k} \subset(V \otimes \mathrm{k})^{+} \text {. }
$$

The same properties hold with $V^{\pi}$ for each $V \in \mathcal{M}^{l l f}\left(\dot{\mathbb{U}}_{\tilde{\pi}, \mathrm{A}}\right)$. 
(b) We have $\left(\mathbb{D}_{\mathrm{A}}^{\prime}\right)^{+} \otimes \mathrm{k}=\left(\mathbb{D}_{\mathrm{k}}^{\prime}\right)^{+}, \mathbb{D}_{\mathrm{A}}^{\pi} \otimes \mathrm{k}=\mathbb{D}_{\mathrm{k}}^{\pi}$ and $\left(\mathbb{F}_{\mathrm{A}} \otimes^{\pi} \mathbb{F}_{\mathrm{A}}\right)^{\pi} \otimes \mathrm{k}=\mathbb{F}_{\mathrm{k}}^{\pi} \otimes^{\pi} \mathbb{F}_{\mathrm{k}}$.

2.3.4. Proposition. (a) There are inclusions of flat A-modules $\mathbb{S}_{\mathrm{A}} \subset \mathbb{S}_{\mathrm{A}, i}$ and $\mathbb{S}_{\mathrm{A}}^{\pi} \subset \mathbb{S}_{\mathrm{A}, i}^{\pi}$ for each $i$.

(b) The natural map $\mathbb{E}_{\mathrm{A}, i}^{\pi} \rightarrow \mathbb{S}_{\mathrm{A}, i}^{\pi}$ is surjective for each $i$.

(c) We have $\mathbb{S}_{\mathrm{A}} \otimes \mathrm{k}=\mathbb{S}_{\mathrm{A}}$ and $\mathbb{S}_{\mathrm{A}}^{\pi} \otimes \mathrm{k}=\mathbb{S}_{\mathrm{k}}^{\pi}$.

(d) The A-modules $\mathbb{R}_{\mathrm{A}}, \mathbb{R}_{\mathrm{A}}^{\pi}$ share the same properties as $\mathbb{S}_{\mathrm{A}}, \mathbb{S}_{\mathrm{A}}^{\pi}$.

Let $\mathbb{J}_{\mathrm{A}} \subset \mathbb{S}_{\mathrm{A}}^{\pi}$ be the left ideal generated by $\partial_{c}\left(\mathbb{U}_{\mathrm{A}}^{\prime}\right)^{\bar{\chi}}$, where $\chi$ is as in 2.2.6(c). Consider the $\dot{\mathbb{U}}_{\mathrm{A}}$-equivariant $\mathbb{S}_{\mathrm{A}}^{\pi}$-module

$$
\mathbb{T}_{\mathrm{A}}=\mathbb{S}_{\mathrm{A}}^{\pi} / \mathbb{J}_{\mathrm{A}}
$$

Taking semi-invariants we define

$$
\mathbb{S}_{\mathrm{A}}^{\pi,+}=\left(\mathbb{S}_{\mathrm{A}}^{\pi}\right)^{+}, \quad \mathbb{T}_{\mathrm{A}}^{+}=\left(\mathbb{T}_{\mathrm{A}}\right)^{+} .
$$

We do not know if these A-modules are flat, but the following is enough for our purposes. It is proved in section A.11.

2.3.6. Proposition. (a) We have $\mathbb{T}_{\alpha} \otimes \mathrm{k}=\mathbb{T}_{\mathrm{k}}$.

(b) We have $\mathbb{S}_{\mathrm{A}}^{\pi,+} \otimes \mathrm{k}=\mathbb{S}_{\mathrm{k}}^{\pi,+}$.

(c) Assume that $p$ is large enough. The $\mathrm{A}_{c}$-modules $\mathbf{T}_{c}, \mathbf{T}_{c}^{+}$are flat. We have isomorphisms of graded $\mathrm{k}_{c}$-vector spaces $\mathbf{T}_{c}^{+} \otimes \mathrm{k}_{c}=\mathbf{T}_{\mathbf{k}_{c}}^{+}$.

2.4. QDO on $\mathbb{P}^{n-1}$ at roots of unity. Let $(\mathrm{A}, \mathrm{k})$ be a modular pair. We will assume that $l$ is finite and $\mathrm{k}$ is a field. This section is a first step towards section 2.7. Here we introduce a coherent sheaf of algebra $\mathfrak{R}$ over a scheme closely related to $T^{*} \mathbb{P}^{n-1}$. This sheaf is a quantum analogue of the ring of differential operators over $\mathbb{P}^{n-1}$ in positive characteristic. First, we need more notation. Set

$$
\begin{aligned}
& \mathbb{A}^{n}=\operatorname{Spec} \mathrm{A}\left[v_{1}, \ldots, v_{n}\right], \\
& \mathbb{A}^{n, *}=\operatorname{Spec} \mathrm{A}\left[\varphi_{1}, \ldots, \varphi_{n}\right], \\
& \mathbb{A}_{\diamond}^{n}=\left\{v \in \mathbb{A}^{n} ; v_{1}+1 \neq 0\right\} .
\end{aligned}
$$

Set $T^{*} \mathbb{A}^{n}=\mathbb{A}^{n} \times \mathbb{A}^{n, *}$. A point in $T^{*} \mathbb{A}^{n}$ will be indicated by the symbol $(v, \varphi)$. Consider the following A-schemes:

$$
\begin{aligned}
& \bar{R}_{\pi}=\left\{(v, \varphi) \in T^{*} \mathbb{A}^{n} ; 1+\sum_{i} v_{i} \varphi_{i} \neq 0\right\}, \\
& R_{\pi}=\left\{(v, \varphi) \in \bar{R}_{\pi} ; \varphi \neq 0\right\}, \\
& R=G \times \mathbb{A}_{\diamond}^{n} .
\end{aligned}
$$

Note that there is a map

$$
m_{R}: \bar{R}_{\pi} \rightarrow G,(v, \varphi) \mapsto(e+v \otimes \varphi)^{-1} .
$$

Equip $\mathbb{A}^{n}$ with the obvious $G$-action and $\mathbb{A}^{n, *}$ with the dual one. Let $G_{\pi} \subset G$ be the isotropy subgroup of $v_{1}$. There is a $G$-action on $\bar{R}_{\pi}$ and a $G_{\pi} \times G$-action on $R$ given by

$$
h \triangleright(v, \varphi)=\left(h v, \varphi h^{-1}\right), \quad\left(h^{\prime}, h\right) \triangleright(g, v)=\left(h^{\prime} g h^{-1}, h^{\prime} v\right) .
$$

Note that $\mathcal{I}_{R}=\mathcal{D} \cap \mathbf{I}_{R}$ is an ideal of $\mathcal{D}$. Set $\mathcal{R}=\mathcal{D} / \mathcal{I}_{R}$. The $\dot{\mathbf{U}}_{\pi}^{\circ} \otimes \dot{\mathbf{U}}$-action on $\mathbf{R}$ yields a $G_{\pi} \times G$-action on $\mathcal{R}$ by $2.2 .3(b)$. Set $\mathcal{R}^{\pi}=\mathcal{R}^{G_{\pi}}$. The canonical map $\mathbf{D} \rightarrow \mathbf{R}$ yields inclusions

$$
\mathcal{R} \subset \mathbf{R}, \quad \mathcal{R}^{\pi} \subset \mathbf{R}^{\pi} .
$$


The following is proved in section A.12.

2.4.3. Proposition. (a) The map $\mathfrak{z}_{D}$ yields a $G_{\pi} \times G$-algebra isomorphism $\mathfrak{z}_{R}$ : $\mathcal{O}(R) \rightarrow \mathcal{R}$. Further, $\bar{R}_{\pi} \simeq \operatorname{Spec}\left(\mathcal{R}^{\pi}\right)$ and $R$ is a $G_{\pi}$-torsor over $R_{\pi}$.

(b) We have $\partial_{\triangleright \mathfrak{z}_{U^{\prime}}}(\mathcal{O}(G)) \subset \mathcal{O}\left(\bar{R}_{\pi}\right)$. This yields an algebra homomorphism $\mu_{R}: \mathcal{O}(G) \rightarrow \mathcal{O}\left(\bar{R}_{\pi}\right)$. It is the comorphism of the map $m_{R}$.

(c) The $\mathcal{O}(R)$-module $\mathbf{R}$ is free of rank $l^{n^{2}+n}$. The $\mathcal{O}\left(\bar{R}_{\pi}\right)$-module $\mathbf{R}^{\pi}$ is free of rank $l^{2 n}$.

The map $\partial_{\triangleleft} \circ \iota$ is a QMM for the $\mathbf{u}_{\pi}$-action on $\mathbf{D}_{R}=\mathbf{D} \otimes_{\mathcal{O}(D)} \mathcal{O}(R)$. The $\mathcal{O}(R)$ algebra $\mathbf{D}_{R} / / \mathbf{u}_{\pi}$ is $G_{\pi} \times \dot{\mathbf{U}}$-equivariant. Faithfully flat descent yields an equivalence

$$
\boldsymbol{M} \operatorname{Mod}\left(\mathcal{O}(R), G_{\pi} \times \dot{\mathbf{U}}\right) \rightarrow \mathcal{Q} \operatorname{coh}\left(\mathcal{O}_{R_{\pi}}, \dot{\mathbf{U}}\right) .
$$

Let $\Re$ be the image of $\mathbf{D}_{R} / / \mathbf{u}_{\pi}$ by this equivalence. It is a quasi-coherent sheaf of $\mathcal{O}_{R_{\pi}}$-algebras over $R_{\pi}$. We define the open subset

$$
R_{\pi, \Sigma}=\left\{(v, \varphi) \in R_{\pi} ; m_{R}(v, \varphi) \in G_{\Sigma}, \varphi_{i} \neq 0\right\} .
$$

The following is proved in section A.12.

2.4.4. Proposition. The sheaf of $\mathcal{O}_{R_{\pi}}$-modules $\mathfrak{R}$ is coherent. If $\mathrm{A}=\mathbb{C}$, it gives an Azumaya algebra of PI-degree $l^{n}$ over $R_{\pi, \Sigma}$.

2.4.5. Remark. The $\mathbb{Z}$-grading on $\mathbb{R}^{\pi}$ in $1.9 .2(b)$ yields the grading on $\mathcal{O}\left(\bar{R}_{\pi}\right)$ associated with the $\mathbb{G}_{m}$-action on $\bar{R}_{\pi}$ given by $z \cdot(v, \varphi)=\left(z^{-1} v, z \varphi\right)$. Note that we have $\operatorname{deg}\left(\varphi_{i}\right)=1$.

2.5. QDO on $G \times \mathbb{P}^{n-1}$ at roots of unity. Let $(\mathrm{A}, \mathrm{k})$ be a modular pair. We will assume that $l$ is finite and $\mathrm{k}$ is a field. This section is the second step towards section 2.7. Using the results from section 2.4 we introduce a coherent sheaf of algebra $\mathfrak{S}_{\pi}$ over a scheme closely related to $T^{*}\left(G \times \mathbb{P}^{n-1}\right)$. This sheaf is a quantum analogue of the ring of differential operators over $G \times \mathbb{P}^{n-1}$ in positive characteristic.

Consider the A-schemes

$$
S=D \times R, \quad \bar{S}_{\pi}=D \times \bar{R}_{\pi}, \quad S_{\pi}=D \times R_{\pi}, \quad S_{\pi, \Sigma}=D_{\Sigma} \times R_{\pi, \Sigma} .
$$

By 2.2.3(c), 2.4.3(a) the maps $\mathfrak{z}_{D}, \mathfrak{z}_{R}$ yield the following A-scheme isomorphisms

$$
\operatorname{Spec}(\mathcal{S}) \rightarrow S, \quad \operatorname{Spec}\left(\mathcal{S}^{\pi}\right) \rightarrow \bar{S}_{\pi},
$$

where $\mathcal{S}=\mathcal{R} \otimes \mathcal{D}$ and $\mathcal{S}^{\pi}=\mathcal{R}^{\pi} \otimes \mathcal{D}$.

By 2.2.6(b) we have an A-linear isomorphism $\mathbf{R} \otimes \mathbf{D}^{\prime} \rightarrow \mathbf{S}$. Composing it with the injections $\mathcal{O}(R) \subset \mathbf{R}$ and $\mathcal{O}(D) \subset \mathbf{D}^{\prime}$ in 2.4.2 and 2.2.3(d) we get the inclusion

$$
\mathcal{O}(S) \subset \mathbf{S} \text {. }
$$

Recall that the general construction in 1.5.2 yields associative multiplications on $\mathbf{S}^{\mathbf{u}_{\pi}}, \mathbf{S}^{\pi}$. By A.7.4(c), (A.8.5) the map (2.5.2) gives $\dot{\mathbf{U}}$-algebra embeddings

$$
\mathcal{O}(S) \subset \mathbf{S}^{\mathbf{u}_{\pi}}, \quad \mathcal{O}\left(\bar{S}_{\pi}\right) \subset \mathbf{S}^{\pi} .
$$

Note that for the $\dot{\mathbf{U}}$-action there is the adjoint one. Note also that

$$
\mathcal{O}(S) \subset Z\left(\mathbf{S}^{\mathbf{u}_{\pi}}\right), \quad \mathcal{O}\left(\bar{S}_{\pi}\right) \subset Z\left(\mathbf{S}^{\pi}\right) .
$$

Next, the $\dot{\mathbf{U}}$-action on $\mathbf{S}^{\mathbf{u}_{\pi}}$ factors to a $G$-action on $\mathcal{O}(S)$ by 2.2.3(b), and the $G_{\pi}$-action on $\mathbf{S}^{\mathbf{u}_{\pi}}$ factors to a $G_{\pi}$-action on $\mathcal{O}(S)$. So $\mathcal{O}(S)$ is a $G_{\pi} \times G$-algebra and $\mathbf{S}^{\mathbf{u}_{\pi}}$ is a $G_{\pi} \times \dot{\mathbf{U}}$-equivariant $\mathcal{O}(S)$-algebra. 
Further, we have a $G_{\pi}$-torsor $S \rightarrow S_{\pi}$ by $2.4 .3(a)$. Thus faithfully flat descent yields an equivalence of categories

$$
\operatorname{Mod}\left(\mathcal{O}(S), G_{\pi} \times \dot{\mathbf{U}}\right) \simeq \mathcal{Q} \operatorname{coh}\left(\mathcal{O}_{S_{\pi}}, \dot{\mathbf{U}}\right)
$$

This equivalence takes $\mathbf{S}^{\mathbf{u}_{\pi}}$ to a quasi-coherent sheaf $\mathfrak{S}_{\pi}$ of $\dot{\mathbf{U}}$-algebras over $S_{\pi}$.

For future use, note that the $G$-action on $\bar{S}_{\pi}$ is given by

$$
h \triangleright\left(g, g^{\prime}, v, \varphi\right)=\left(h g h^{-1}, h g^{\prime} h^{-1}, h v, \varphi h^{-1}\right) .
$$

For $s \in \mathcal{O}\left(\bar{S}_{\pi}\right)$ let $S_{\pi, s}=\{s \neq 0\}$ be an affine open subset of $\bar{S}_{\pi}$. Put

$$
S_{\pi, \diamond}=\bigcup_{s} S_{\pi, s},
$$

where $s$ runs over the set of semi-invariant elements in $\mathcal{O}\left(\bar{S}_{\pi}\right)^{+}$which are homogeneous of positive degree. The following is proved in section A.13.

2.5.6. Proposition. (a) The $\mathcal{O}_{S_{\pi}}$-module $\mathfrak{S}_{\pi}$ is coherent.

(b) The $\dot{\mathbf{U}}$-modules $\mathfrak{S}_{\pi}\left(S_{\pi}\right)$ and $\mathfrak{S}_{\pi}\left(S_{\pi, \diamond}\right)$ are locally finite.

$(c)$ Let $\mathrm{A}=\mathbb{C}$. Then $S_{\pi, \diamond}(\mathbb{C})$ is the set of tuples $\left(g, g^{\prime}, v, \varphi\right) \in S_{\pi}(\mathbb{C})$ such that there is no proper subspace of $\mathbb{C}^{n, *}$ containing $\varphi$ and preserved by $g, g^{\prime}$. The $G$-action on $S_{\pi, \diamond}$ is free.

(d) Let $\mathrm{A}=\mathbb{C}$. The sheaf $\mathfrak{S}_{\pi}$ restricts to an Azumaya algebra over $S_{\pi, \Sigma}$.

(e) Let $\mathrm{A}=\mathbb{C}$. The variety $\bar{S}_{\pi}$ is a Poisson $G$-variety. The sheaf $\mathfrak{S}_{\pi}$ is a Poisson order over $S_{\pi}$. For each $G$-invariant open subset $U \subset S_{\pi}$ the algebra $\mathfrak{S}_{\pi}(U)^{0}$ is a Poisson order over $\mathcal{O}(U)^{0}$.

2.5.7. Remarks. (a) If $\tau=1$, then $\mathbf{S}^{\pi}=\mathcal{O}\left(\bar{S}_{\pi}\right)$.

(b) By 1.10.3(b) and 2.2.3(a) the map $\partial_{c}$ factors to $\mathbf{U}^{\prime} \rightarrow \mathbf{S}^{\pi}$. By (2.5.3) we have an inclusion $\mathcal{O}\left(\bar{S}_{\pi}\right)=\mathcal{R}^{\pi} \otimes \mathcal{D} \subset \mathbf{S}^{\pi}$. By A.7.4(b), 1.3.3(c) and (A.8.7) the map $\partial_{c}$ factors to an algebra homomorphism

$$
\partial_{c}: \mathcal{U}^{\prime} \rightarrow \mathcal{O}\left(\bar{S}_{\pi}\right), \kappa(f) \mapsto \partial_{\triangleright} \kappa\left(f_{1}\right) \otimes \dagger\left(\partial_{\triangleleft} \kappa \iota\left(f_{3}\right) \partial_{\triangleright} \kappa\left(f_{2}\right)\right), \forall f \in \mathcal{F} .
$$

2.6. Definition of the deformed Hilbert scheme. In this section we introduce the deformed Hilbert scheme. Let us denote it by the symbol $T$. It is one of the main objects in the paper. We define also a Poisson bracket on $T$. Most properties of $T$ will be proved later, in section 4.1. In particular, we will prove there that $T$ is smooth, connected and symplectic. We will use some of these properties before section 4.1, hoping it will not create any confusion.

Let $(\mathrm{A}, \mathrm{k})$ be a modular pair. We will assume that $l$ is finite and $\mathrm{k}$ is a field. Fix $c \in \mathbb{Q}^{\times}$. Recall that a $\mathrm{A}_{b}$-scheme is indicated by the subscript $b$ and a $\mathrm{A}_{c}$-scheme by the subscript $c$. For instance, $T$ yields the schemes $T_{b}, T_{c}$.

Set $[g, h]=g^{-1} h g h^{-1}$ for each $g, h \in G$. We have the A-scheme homomorphism

$$
m_{S}: \bar{S}_{\pi} \rightarrow G, x=\left(g, g^{\prime}, v, \varphi\right) \mapsto\left[g, g^{\prime}\right](e+v \otimes \varphi) .
$$

Let $T_{\pi} \subset \bar{S}_{\pi}$ be the Zariski closure of the ideal $\left(m_{S}-\zeta^{2 l} e\right) \subset \mathcal{O}\left(\bar{S}_{\pi}\right)$. Recall that

$$
\bar{S}_{\pi}=D \times \bar{R}_{\pi} \subset D \times T^{*} \mathbb{A}^{n} .
$$

The $G$-action on $\bar{S}_{\pi}$ preserves the subscheme $T_{\pi}$ by (2.5.4). By (2.3.2) we have subalgebras

$$
\mathcal{O}\left(T_{\pi}\right)^{+}, \mathcal{O}\left(T_{\pi}\right)^{0} \subset \mathcal{O}\left(T_{\pi}\right)
$$


Both are affine $\mathbb{Z}_{+}$-graded A-algebras by $[\underline{\mathrm{S}}$, thm. II.4.2(i)]. For future use, note that we have

$$
\mathcal{O}\left(T_{\pi}\right)^{+}=\bigoplus_{m \geqslant 0}\left\{f \in \mathcal{O}\left(T_{\pi}\right) ; f(h x)=(\operatorname{det} h)^{m} f(x), \forall h, x\right\} .
$$

Now we can define the projective morphism

$$
\mathfrak{q}: T \rightarrow N, \quad T=\operatorname{Proj}\left(\mathcal{O}\left(T_{\pi}\right)^{+}\right), \quad N=\operatorname{Spec}\left(\mathcal{O}\left(T_{\pi}\right)^{0}\right) .
$$

We call $T$ the deformed Hilbert scheme. See section 4.1 for more details on $T$.

Let $\mathcal{T}=\mathcal{S}^{\pi} / \mathcal{J}$, where $\mathcal{J} \subset \mathcal{S}^{\pi}$ is the ideal generated by $\partial_{c}\left(\mathcal{U}^{\prime}\right)^{\bar{\chi}}$. It is an affine $G$-algebra.

2.6.2. Proposition. (a) We have $\mathcal{T} \simeq \mathcal{O}\left(T_{\pi}\right)$.

(b) If $\mathrm{A}=\mathbb{C}$, then $T$ is a smooth symplectic variety.

(c) The $\mathrm{A}_{b}$-schemes $T_{\pi, b}, T_{b}$ and $N_{b}$ are integral and flat. We have $T_{b} \otimes \mathbb{C}=T_{\mathbb{C}}$.

(d) Assume that $p$ is large enough. The $\mathrm{A}_{c}$-schemes $T_{\pi, c}, T_{c}$ and $N_{c}$ are integral and flat. We have $T_{c} \otimes \mathrm{k}_{c}=T_{\mathrm{k}_{c}}^{(e)}$.

Proof. (a) Composing $\mathfrak{z} U^{\prime}, \partial_{c}$ and (2.5.1) yields an algebra homomorphism

$$
\mu_{S}: \mathcal{O}(G) \rightarrow \mathcal{O}\left(\bar{S}_{\pi}\right), f \mapsto f_{5} \iota\left(f_{3}\right) \otimes f_{2} \iota\left(f_{4}\right) \otimes \mu_{R}\left(f_{1}\right), \forall f \in \mathcal{O}(G) ;
$$

see 2.5.7(b). It is the comorphism of the map

$$
\bar{S}_{\pi} \rightarrow G, \quad x \mapsto m_{S}(x)^{-1} .
$$

Thus 2.2.6(d) yields an isomorphism $\mathcal{T} \simeq \mathcal{O}\left(T_{\pi}\right)$.

(b) Assume that $\mathrm{A}=\mathbb{C}$. Let us construct a Poisson bracket on $T$. The smoothness of $T$ and nondegeneracy of the bracket we will be proved in 4.1.1. For each homogeneous element $t \in \mathcal{O}\left(T_{\pi}\right)^{+}$of degree $>0$ we set

$$
T_{\pi, t}=\left\{x \in T_{\pi} ; t(x) \neq 0\right\}, \quad T_{t}=T_{\pi, t} / G .
$$

We have $T=\bigcup_{t} T_{t}$, an affine open covering. The comorphism of the closed embed$\operatorname{ding} T_{\pi} \subset \bar{S}_{\pi}$ is a surjective map

$$
\mathcal{O}\left(\bar{S}_{\pi}\right) \rightarrow \mathcal{O}\left(T_{\pi}\right)
$$

Taking $G$-invariants is an exact functor over $\mathbb{C}$. Thus the restriction to $T_{\pi}$ yields a surjective map

$$
\mathcal{O}\left(\bar{S}_{\pi}\right)^{+} \rightarrow \mathcal{O}\left(T_{\pi}\right)^{+}
$$

Fix a homogeneous element $s \in \mathcal{O}\left(\bar{S}_{\pi}\right)^{+}$which maps to $t$. Recall that $\mathcal{O}\left(S_{\pi, s}\right)$ is a Poisson $G$-algebra by 2.5.6(d). Thus $\mathcal{O}\left(S_{\pi, s}\right)^{0}$ is again a Poisson algebra. Now, the map (2.6.4) yields a surjective algebra homomorphism

$$
\mathcal{O}\left(S_{\pi, s}\right)^{0} \rightarrow \mathcal{O}\left(T_{t}\right)
$$

whose kernel is generated by $\partial_{c}\left(\mathcal{U}^{\prime}\right)^{\bar{\chi}}$. We claim that there is a unique Poisson bracket on $\mathcal{O}\left(T_{t}\right)$ such that (2.6.5) is a Poisson algebra homomorphism.

We have $\mathbb{C}=\mathcal{A} /(q-\tau)$ where $\mathcal{A}=\mathbb{C}\left[q^{ \pm 1}\right]$. Consider the $\mathbb{C}$-linear map

$$
\sigma_{U}: \mathbf{U} \rightarrow \mathbb{U}_{\mathcal{A}}
$$

taking $\dot{e}^{m} k_{\lambda} \iota\left(\dot{f}^{n}\right)$ to itself for each $m, n, \lambda$. There is a unique $\mathbb{C}$-linear map

$$
\nu: \mathcal{U} \rightarrow \dot{\mathbf{U}}, \quad u \mapsto \nu(u)
$$


such that $\nu(u)$ is the specialization of the element

$$
\left(\sigma_{U}(u)-\varepsilon(u)\right) / \hbar \in \dot{\mathbb{U}}_{\mathcal{A}}, \quad \hbar=l\left(q^{l}-q^{-l}\right) .
$$

Next, recall that $\mathcal{O}\left(\bar{S}_{\pi}\right) \subset \mathbf{S}^{\pi}$ by (2.5.3) and that $\mathbf{S}^{\pi}=\mathbb{S}_{\mathcal{A}}^{\pi} \otimes \mathbb{C}$ by 2.3.4(c). Thus any element of $\mathcal{O}\left(\bar{S}_{\pi}\right)$ lifts to $\mathbb{S}_{\mathcal{A}}^{\pi}$. Let $\{$,$\} denote the Poisson bracket on \mathcal{O}\left(\bar{S}_{\pi}\right)$ constructed in 2.5.6(e). The map $\partial_{c}: \mathbb{U}_{\mathcal{A}}^{\prime} \rightarrow \mathbb{S}_{\mathcal{A}}^{\pi}$ factors to $\mathcal{U}^{\prime} \rightarrow \mathcal{O}\left(\bar{S}_{\pi}\right)$ by $2.5 .7(b)$. A routine computation using (1.5.1) yields

$$
\left\{\partial_{c}(u), x\right\}=\left(\operatorname{ad} \nu\left(u_{1}\right)\right)(x) \partial_{c}\left(u_{2}\right), \quad \forall x \in \mathcal{O}\left(\bar{S}_{\pi}\right), u \in \mathcal{U}^{\prime} .
$$

Compare (2.1.2). Consider the $\mathbb{C}$-linear map

$$
\gamma: \mathbf{U} \rightarrow \mathbb{C}, \quad \dot{e}^{m} k_{\lambda} \iota\left(\dot{f}^{n}\right) \mapsto \lambda \cdot \omega_{n} \text { if } m=n=0, \quad 0 \text { otherwise. }
$$

A short computation using (2.3.1) yields

$$
\langle m\rangle \nu(u)=m \gamma(u) / 2 l, \quad \forall u \in \mathcal{U}, m \in \mathbb{Z} .
$$

Hence (2.3.2) yields

$$
(\operatorname{ad} \nu(u))(x)=m \gamma(u) x / 2 l, \quad \forall u \in \mathcal{U}, x \in \mathbf{S}^{\pi, m}, m \in \mathbb{Z} .
$$

Therefore, from (2.6.8) and the Leibniz rule we get

$$
\left\{\partial_{c}(u), \mathcal{O}\left(S_{\pi, s}\right)^{0}\right\}=0, \quad \forall u \in \mathcal{U}^{\prime} .
$$

Hence the Poisson bracket on $\mathcal{O}\left(S_{\pi, s}\right)^{0}$ factors to $\mathcal{O}\left(T_{t}\right)$ by $(2.6 .5)$.

(c) Now we set $\mathrm{A}=\mathrm{A}_{b}$. Let us prove that $T_{\pi, b}$ is an integral scheme. First, we prove that the $\mathrm{A}_{b}$-module $\mathcal{O}\left(T_{\pi, b}\right)$ is flat. Recall that $\mathrm{A}_{b}$ is a DVR with residue field $\mathbb{C}$. We must check that the $\mathrm{A}_{b}$-module $\mathcal{O}\left(T_{\pi, b}\right)$ is torsion-free. If it was false there should be an irreducible component of $T_{\pi, b}$ not dominating $\operatorname{Spec}\left(\mathrm{A}_{b}\right)$. This component would be a $\mathbb{C}$-scheme of dimension $>2 n+n^{2}$ because $T_{\pi, b}$ is given by $n^{2}$ equations and $\operatorname{dim}\left(\bar{S}_{\pi, b}\right)=2 n^{2}+2 n+1$. On the other hand, since taking tensor products is right exact we have

$$
T_{\pi, b} \otimes \mathbb{C}=T_{\pi, \mathbb{C}}
$$

Further, all irreducible components of $T_{\pi, \mathbb{C}}$ have dimension $2 n+n^{2}$ by $4.1 .1(b)$ below. This yields a contradiction.

Next, since $\mathcal{O}\left(T_{\pi, b}\right)$ is a flat $\mathrm{A}_{b}$-module we have $\mathcal{O}\left(T_{\pi, b}\right) \subset \mathcal{O}\left(T_{\pi, \mathrm{k}_{b}}\right)$. Thus $\mathcal{O}\left(T_{\pi, b}\right)$ is a CID by $4.1 .1(b)$.

As $N_{b}$ is the categorical quotient of $T_{\pi, b}$ it is also integral. For a similar reason $T_{b}$ is integral. Finally, we must prove that $T_{b} \otimes \mathbb{C}=T_{\mathbb{C}}$. Recall that $T=\operatorname{Proj}\left(\mathcal{O}\left(T_{\pi}\right)^{+}\right)$. Thus the claim follows from (2.6.9) because taking $G$-semi-invariants over $\mathbb{C}$ is an exact functor.

(d) Now we set $\mathrm{A}=\mathrm{A}_{c}$. Assume that $p$ is large enough. Let us prove that $T_{\pi, c}$ is an integral scheme. Assume temporarily that $\mathrm{A}=\mathbb{Z}[t]$ and $\zeta=t$. By generic flatness there is an element $0 \neq f(t) \in \mathrm{A}$ such that the $\mathrm{A}_{f}$-module $\mathcal{O}\left(T_{\pi}\right)_{f}$ is flat. We may assume that $f(\zeta) \neq 0$ in $\mathrm{k}_{c}$ because $p$ is large. Thus $f(\zeta)$ is invertible in $\mathrm{A}_{c}$. Hence the morphism $T_{\pi, c} \rightarrow \operatorname{Spec}\left(\alpha_{c}\right)$ is flat. Since $\mathrm{k}_{c} \subset \mathbb{C}$ this implies that

$$
\mathcal{O}\left(T_{\pi, c}\right) \subset \mathcal{O}\left(T_{\pi, \mathbb{C}}\right) \text {. }
$$

Now $\mathcal{O}\left(T_{\pi, \mathbb{C}}\right)$ is a CID by part $(c)$. Hence $T_{\pi, c}$ is an integral scheme. 
Observe that $\mathbf{S}_{c}^{\pi} \otimes \mathrm{k}_{c}=\mathcal{S}_{\mathrm{k}_{c}}^{\pi}$ by 2.3.4(c). Recall that $\mathcal{S}_{c}^{\pi}=\mathcal{R}_{c}^{\pi} \otimes \mathcal{D}_{c}$ and recall the notation from (1.2.1) and (1.2.2). It is easy to see that the natural map $\mathcal{S}_{c}^{\pi} \subset \mathbf{S}_{c}^{\pi}$ yields isomorphisms

$$
\mathcal{S}_{c}^{\pi} \otimes \mathrm{k}_{c}=\left(\mathcal{S}_{\mathrm{k}_{c}}^{\pi}\right)^{l}, \quad \mathcal{J}_{c} \otimes \mathrm{k}_{c}=\left(\mathcal{J}_{\mathrm{k}_{c}}\right)^{[l]} .
$$

Therefore, there is an isomorphism of $\mathrm{k}_{c}$-algebras

$$
\mathcal{T}_{c} \otimes \mathrm{k}_{c}=\left(\mathcal{S}_{c}^{\pi} \otimes \mathrm{k}_{c}\right) /\left(\mathcal{J}_{c} \otimes \mathrm{k}_{c}\right)=\left(\mathcal{S}_{\mathrm{k}_{c}}^{\pi}\right)^{l} /\left(\mathcal{J}_{\mathrm{k}_{c}}\right)^{[l]} .
$$

Therefore, since $T_{\pi, c}$ is reduced, part $(a)$ and (1.2.2) yield an isomorphism of $\mathrm{k}_{c^{-}}$ algebras

$$
\mathcal{O}\left(T_{\pi, c}\right) \otimes \mathrm{k}_{c}=\mathcal{O}\left(T_{\pi, \mathrm{k}_{c}}\right)^{l} .
$$

So there is a $\mathrm{k}_{c}$-scheme isomorphism

$$
T_{\pi, c} \otimes \mathrm{k}_{c} \rightarrow\left(T_{\pi, \mathrm{k}_{c}}\right)^{(e)} .
$$

The rest follows as in part $(c)$, taking $G$-semi-invariants, because $p$ is large.

2.6.11. Remarks. (a) If $\tau=1$, then $\mathbf{T}=\mathcal{O}\left(T_{\pi}\right)$.

(b) Note that $\mathcal{O}\left(T_{\pi}\right)^{0}$ is a Poisson algebra but $\mathcal{O}\left(T_{\pi}\right)^{+}$is not. Indeed, the homogeneous coordinate ring of a projective Poisson variety may not be a Poisson algebra.

(c) The variety $T_{\pi}$ appears already in $[\mathrm{O}$, sec. 1.2$]$ where it is denoted $C M_{\zeta^{l}}^{\prime}$.

2.7. QDO over the deformed Hilbert scheme. Let $(\mathrm{A}, \mathrm{k})$ be a modular pair. Assume that $l$ is finite. In this section we construct a coherent sheaf of $\mathcal{O}_{T}$-algebras $\mathfrak{T}$ over the deformed Hilbert scheme $T$. This is done by quantum reduction from the sheaf of $\dot{\mathbf{U}}$-algebras $\mathfrak{S}_{\pi}$ over $S_{\pi}$. We will prove later, in section 4.2 , that $\mathfrak{T}$ is an Azumaya algebra over $T$. Here we will check that it is a Poisson order over $T$ and that it behaves nicely under base change.

Recall the $\dot{\mathbf{U}}$-equivariant $\mathbf{S}^{\pi}$-module $\mathbf{T}=\mathbf{S}^{\pi} / \mathbf{J}$ in (2.3.5). The general construction in (2.3.2) yields A-submodules

$$
\mathbf{T}^{0}, \mathbf{T}^{+} \subset \mathbf{T} .
$$

2.7.1. Lemma. The following hold:

(a) $\mathbf{T}$ is a $\dot{\mathbf{U}}$-equivariant $\mathcal{O}\left(T_{\pi}\right)$-module of finite type,

(b) $\mathbf{T}^{\mathbf{u}}$ is a $G$-equivariant $\mathcal{O}\left(T_{\pi}\right)$-algebra and a $\mathcal{O}\left(T_{\pi}\right)$-module of finite type,

(c) $\mathbf{T}^{+}$is a $\mathbb{Z}_{+}$-graded $\mathcal{O}\left(T_{\pi}\right)^{+}$-module of finite type,

(d) $\mathbf{T}^{0}$ is a $\mathcal{O}(N)$-algebra and a $\mathcal{O}(N)$-module of finite type.

Proof. (a) By 2.6.2(a) we have $\mathcal{O}\left(T_{\pi}\right)=\mathcal{O}\left(\bar{S}_{\pi}\right) / \mathcal{J}$. Further, (2.5.3) yields a $\dot{U}-$ algebra homomorphism $\mathcal{O}\left(\bar{S}_{\pi}\right) \rightarrow \mathbf{S}^{\pi}$ which maps $\mathcal{J}$ into $\mathbf{J}$. Thus the $\mathbf{S}^{\pi}$-action on $\mathbf{T}$ yields a $\dot{\mathbf{U}}$-equivariant $\mathcal{O}\left(T_{\pi}\right)$-action on $\mathbf{T}$.

(b) Equip $\mathbf{T}^{\mathbf{u}}$ with the multiplication in 1.5.2. Note that $\mathbf{T}^{\mathbf{u}} \subset \mathbf{T}$ is a $\dot{\mathbf{U}}$ submodule because $\mathbf{u} \subset \dot{\mathbf{U}}$ is a normal Hopf subalgebra. The $\dot{\mathbf{U}}$-action on $\mathbf{T}^{\mathbf{u}}$ factors to a locally finite $\mathfrak{U}$-action. Thus $\mathbf{T}^{\mathbf{u}}$ is a $G$-algebra. It is also a $\mathcal{O}\left(T_{\pi}\right)$ module by part $(a)$. So it is a $G$-equivariant $\mathcal{O}\left(T_{\pi}\right)$-algebra. Finally, $\mathbf{T}^{\mathbf{u}}$ is a finitely generated $\mathcal{O}\left(T_{\pi}\right)$-module by part $(a)$ again, because $\mathcal{O}\left(T_{\pi}\right)$ is Noetherian.

$(c),(d)$ Use [S, Thm. II.4.2(ii)]. 
Next, there are localization functors

$$
\boldsymbol{M o d}\left(\mathcal{O}\left(T_{\pi}\right), G\right) \rightarrow \mathcal{Q} \operatorname{coh}\left(\mathcal{O}_{T_{\pi}}, G\right), \quad \mathcal{G} \boldsymbol{r}\left(\mathcal{O}\left(T_{\pi}\right)^{+}\right) \rightarrow \mathcal{Q} \operatorname{coh}\left(\mathcal{O}_{T}\right) .
$$

Let $\mathfrak{T}_{\pi}, \mathfrak{T}$ be the images of $\mathbf{T}^{\mathbf{u}}, \mathbf{T}^{+}$respectively by (2.7.2). They are coherent sheaves by $2.7 .1(b),(c)$.

2.7.3. Proposition. Fix $c \in \mathbb{Q}^{\times}$.

(a) The $\mathcal{O}_{T_{c}}$-module $\mathfrak{T}_{c}$ is $\mathrm{A}_{c}$-flat.

(b) Assume that $p$ is large enough and that $(\tau, \zeta) \in \Gamma_{c}(\mathbb{C})$. The natural $\mathcal{O}_{T_{\mathbb{C}}}$ module homomorphism $\mathfrak{T}_{b} \otimes \mathbb{C} \rightarrow \mathfrak{T}_{\mathbb{C}}$ is surjective.

Proof. Recall that the sets $T_{t}$ in (2.6.3) form an affine open cover of $T$ with

$$
\mathfrak{T}\left(T_{t}\right)=\mathbf{T}_{(t)} .
$$

(a) The $\mathrm{A}_{c}$-module $\mathbf{T}_{c,(t)}$ is flat by 2.3.4(c). Thus $\mathfrak{T}_{c}$ is also $\mathrm{A}_{c}$-flat.

(b) We must prove that the natural map below is surjective

$$
\mathbf{T}_{b,(t)} \otimes \mathbb{C} \rightarrow \mathbf{T}_{\mathbb{C},(t)} .
$$

Note that both sides are $\mathcal{O}\left(T_{\mathbb{C}, t}\right)$-modules by 2.6.2(c). The restriction of functions yields a surjective algebra homomorphism

$$
\mathcal{O}\left(\bar{S}_{\pi}\right) \rightarrow \mathcal{O}\left(T_{\pi}\right)
$$

Recall that $\mathbf{S}^{\pi}$ is an $\mathcal{O}\left(\bar{S}_{\pi}\right)$-algebra by (2.5.3) and that $\mathbf{T}$ is an $\mathcal{O}\left(T_{\pi}\right)$-module by 2.7.1(a). So $\mathbf{T}$ can be viewed as a $\mathcal{O}\left(\bar{S}_{\pi}\right)$-module via (2.7.4) and the canonical map $\mathbf{S}^{\pi} \rightarrow \mathbf{T}$ is an $\mathcal{O}\left(\bar{S}_{\pi}\right)$-module homomorphism.

If $\mathrm{A}$ is any $\mathbb{C}$-algebra the map (2.7.4) gives a surjective algebra homomorphism

$$
\mathcal{O}\left(\bar{S}_{\pi}\right)^{+} \rightarrow \mathcal{O}\left(T_{\pi}\right)^{+} \text {. }
$$

Compare (2.6.4). Given $t \in \mathcal{O}\left(T_{\pi}\right)^{+}$we may fix a homogeneous element $s \in \mathcal{O}\left(\bar{S}_{\pi}\right)^{+}$ which maps to $t$. The canonical map $\mathbf{S}^{\pi} \rightarrow \mathbf{T}$ yields a map

$$
f: \mathbf{S}_{(s)}^{\pi} \rightarrow \mathbf{T}_{(t)}
$$

which is compatible, in the obvious way, with the surjective Poisson map (2.6.5)

$$
\mathcal{O}\left(S_{\pi, s}\right)^{0} \rightarrow \mathcal{O}\left(T_{t}\right)
$$

Now, we take $\mathrm{A}=\mathrm{A}_{b}$ or $\mathbb{C}$. To unburden the notation, when $\mathrm{A}=\mathbb{C}$ we will omit the subscript $\mathbb{C}$. The maps $f_{\mathrm{b}}, f$ yield a commutative square

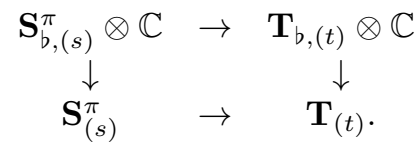

We must prove that the right vertical map is surjective. The left one is invertible by 2.3.6(b). Thus, it is obvious that the map $f$ is surjective. We would like to apply a quantum version of A.11.3. Since we do not know how to do this, we use Poisson orders. More precisely, recall that

$$
\mathbf{S}_{(s)}^{\pi}=\mathfrak{S}_{\pi}\left(S_{\pi, s}\right)^{0}, \quad \mathbf{T}_{(t)}=\mathfrak{T}\left(T_{t}\right) .
$$

See (A.13.2). Thus $\mathbf{S}_{(s)}^{\pi}$ is a Poisson order over $\mathcal{O}\left(S_{\pi, s}\right)^{0}$ by 2.5.6(e). Further, $\mathbf{T}_{(t)}$ is a Poisson order over $\mathcal{O}\left(T_{t}\right)$ and $f$ is a Poisson order homomorphism by 2.7.6 below. See section A.10 for more details on Poisson orders. 
Let $M$ be the cokernel of $f$. It is a Poisson order over $T_{t}$ by A.10.3(c). Next $T_{t}$ is a smooth symplectic variety by $2.6 .2(b)$. Thus $M$ is a projective $\mathcal{O}\left(T_{t}\right)$-module by A.10.3(a). Note that $T_{t}$ is connected; see 4.1.1(c). Therefore it is enough to prove that $M$ vanishes at the generic point of $T_{t}$.

By base change it is enough to prove that $M_{c}$ vanishes over the generic point of $T_{c, t}$. We may assume that $s \in \mathcal{O}\left(\bar{S}_{\pi, c}\right)^{+}$and $t \in \mathcal{O}\left(T_{\pi, c}\right)^{+}$. Since $\tau=1$ in $\mathrm{k}_{c}$ and $p$ is large we have

$$
\mathbf{S}_{\mathrm{k}_{c}}^{\pi}=\mathcal{O}\left(\bar{S}_{\pi, \mathrm{k}_{c}}\right), \quad \mathbf{T}_{\mathrm{k}_{c}}=\mathcal{O}\left(T_{\pi, \mathrm{k}_{c}}\right) .
$$

See 2.5.7(a) and 2.7.7 below. Hence $f_{\mathrm{k}_{c}}$ is surjective by A.11.3. By 2.3.6(b), $(c)$ we have

$$
\mathbf{S}_{c,(s)}^{\pi} \otimes \mathrm{k}_{c}=\mathbf{S}_{\mathbf{k}_{c},(s)}^{\pi}, \quad \mathbf{T}_{c,(t)} \otimes \mathrm{k}_{c}=\mathbf{T}_{\mathrm{k}_{c},(t)} .
$$

So $M_{c} \otimes \mathrm{k}_{c}=0$. Observe that $M_{c}$ is a $\mathcal{O}\left(T_{c, t}\right)$-module of finite type and that a module of finite type over a CID which vanishes at some closed point is generically trivial by Nakayama's lemma. We are done.

Recall the set $S_{\pi, \diamond}$ introduced in (2.5.5). There is a canonical map

$$
\mathfrak{p}: T_{\pi, \diamond} \rightarrow T, \quad T_{\pi, \diamond}=T_{\pi} \cap S_{\pi, \diamond} .
$$

Now we assume that $\mathrm{A}=\mathbb{C}$. By 4.1.1( $a$ ) below, the map $\mathfrak{p}$ is a $G$-torsor.

2.7.6. Proposition. Let $\mathrm{A}=\mathbb{C}$. We have $\mathfrak{T}=\mathfrak{p}_{*}\left(\left.\mathfrak{T}_{\pi}\right|_{T_{\pi, \diamond}}\right)^{G}$. The sheaf $\mathfrak{T}$ is a Poisson order over $T$.

Proof. Let $s \in \mathcal{O}\left(\bar{S}_{\pi}\right)^{+}$be homogeneous of positive degree and let $t \in \mathcal{O}\left(T_{\pi}\right)^{+}$be its image by the map (2.6.4). The first claim is obvious, because we have

$$
\mathfrak{T}\left(T_{t}\right)=\mathbf{T}_{(t)}=\mathfrak{T}_{\pi}\left(T_{\pi, t}\right)^{G} .
$$

Let us concentrate on the second claim. We have $\mathbb{C}=\mathcal{A} /(q-\tau)$ where $\mathcal{A}=\mathbb{C}\left[q^{ \pm 1}\right]$. By (2.5.3) we have an algebra embedding

$$
\mathcal{O}\left(\bar{S}_{\pi}\right) \subset Z\left(\mathbf{S}^{\pi}\right)
$$

By 2.3.6(b) we also have

$$
\mathbf{S}^{\pi,+}=\mathbb{S}_{\mathcal{A}}^{\pi,+} \otimes \mathbb{C} .
$$

Fix a $\mathbb{C}$-linear section $\sigma_{S}: \mathcal{O}\left(\bar{S}_{\pi}\right)^{+} \rightarrow \mathbb{S}_{\mathcal{A}}^{\pi,+}$. By A.10.2(b) the commutator in $\mathbb{S}_{\mathcal{A}}^{\pi}$ yields a linear map

$$
\theta: \mathcal{O}\left(\bar{S}_{\pi}\right)^{+} \rightarrow \operatorname{Der}\left(\mathbf{S}^{\pi}\right)
$$

Let $\{$,$\} denote the Poisson bracket on \mathcal{O}\left(\bar{S}_{\pi}\right)$ constructed in 2.5.6(e). We have

$$
\theta(x)\left(x^{\prime}\right)=\left\{x, x^{\prime}\right\}, \quad \forall x, x^{\prime} \in \mathcal{O}\left(\bar{S}_{\pi}\right)^{+} .
$$

By the Leibniz rule the map $\theta$ yields a $\mathbb{C}$-linear map

$$
\theta: \mathcal{O}\left(S_{\pi, s}\right)^{0} \rightarrow \operatorname{Der}\left(\mathbf{S}_{s}^{\pi}\right)
$$

By definition of the Poisson bracket on $\mathcal{O}\left(T_{t}\right)$ the map (2.6.5) is a surjective Poisson algebra homomorphism

$$
\mathcal{O}\left(S_{\pi, s}\right)^{0} \rightarrow \mathcal{O}\left(T_{t}\right)
$$

We must prove that $\theta$ factors also to a linear homomorphism

$$
\theta: \mathcal{O}\left(T_{t}\right) \rightarrow \operatorname{Der}\left(\mathbf{T}_{(t)}\right) .
$$

This is routine and is left to the reader. Compare the proof of 2.6.2(b).

2.7.7. Remark. If $\tau=1$, then $\mathbf{T}=\mathcal{O}\left(T_{\pi}\right)$. 
2.8. The deformed Harish-Chandra homomorphism at roots of unity. Now, we specialize the deformed Harish-Chandra homomorphism $\Phi: \mathbb{T}^{0} \rightarrow \mathbb{D}_{0, \star}^{\Sigma_{n}}$ in 1.11.4 to roots of unity. Set $\mathrm{A}=\mathbb{C}$ and assume that $l$ is finite. Consider the affine open subset $T_{\star}=\mathfrak{p}\left(T_{\pi, \star}\right)$ defined in section 4.1. The main result of this section is the following.

2.8.1. Theorem. Assume that $p$ is large enough.

(a) The deformed Harish-Chandra homomorphism factors to an algebra homomorphism $\mathfrak{T}\left(T_{\star}\right) \rightarrow \mathbf{D}_{0, \star}^{\Sigma_{n}}$.

(b) The deformed Harish-Chandra homomorphism factors also to a Poisson algebra homomorphism $\mathcal{O}\left(T_{\star}\right) \rightarrow \mathcal{D}_{0, \star}^{\Sigma_{n}}$.

Proof. (a) Recall that for each tuple $m, n$ of integers $\geqslant 0$ we have a monomial $u=\dot{e}^{m} k_{\lambda} \iota\left(\dot{f}^{n}\right)$ in $\dot{U}_{\mathcal{A}}$. Set

$$
|u|=|m|-|n|, \quad \operatorname{deg}(u)=\rho \cdot|m|+\rho \cdot|n| .
$$

By [EK] for each $u$ there are monomials $u_{1}, u_{2}, \ldots, u_{r} \in \dot{\mathbb{U}}_{\mathcal{A}}$ and elements $d_{1}, d_{2}, \ldots$, $d_{r} \in \mathbb{U}_{\mathcal{A}} \otimes \mathbb{D}_{0, \mathcal{A}, \star}^{\prime}$ such that

$$
\begin{aligned}
& \operatorname{deg}\left(u_{1}\right), \ldots, \operatorname{deg}\left(u_{r}\right)<\operatorname{deg}(u), \\
& \left(1-q^{-|u| \cdot|m|} \otimes q^{-|m|}\right) \nabla(u)=\sum_{i=1}^{r} d_{i} \nabla\left(u_{i}\right) .
\end{aligned}
$$

Recall that $q^{-|m|}$ is the element $\ell\left(q^{-|m|}\right) \in \mathbb{D}_{0, \mathcal{A}}^{\prime}$. So an induction on $\operatorname{deg}(u)$ implies that

$$
\nabla\left(\mathbb{U}_{\mathcal{A}}\right) \subset \mathbb{U}_{\mathcal{A}} \otimes \mathbb{D}_{0, \mathcal{A}, \star}^{\prime}
$$

Thus the map (1.11.7) factors through a map

$$
\phi: \mathbb{E}_{\mathcal{A}} \rightarrow \mathbb{D}_{\triangleright, \mathcal{A}} \otimes \mathbb{D}_{0, \mathcal{A}, \star}^{\prime} .
$$

It vanishes on the left ideal generated by $\partial_{b} \iota\left(\mathbb{U}_{\pi, \mathcal{A}}^{\prime}\right)^{\bar{\chi}}$ and $\partial_{c}\left(\mathbb{U}_{\mathcal{A}}^{\prime}\right)^{\varepsilon}$.

Now, assume that $(\mathcal{A}, \mathrm{A})$ is any modular pair. By base change we have again an A-linear map

$$
\phi: \mathbb{E}_{\mathrm{A}} \rightarrow \mathbb{D}_{\triangleright, \mathrm{A}} \otimes \mathbb{D}_{0, \mathrm{~A}, \star}^{\prime}
$$

which vanishes on the left ideal generated by $\partial_{b} \iota\left(\mathbb{U}_{\pi, \mathrm{A}}^{\prime}\right)^{\bar{\chi}}$ and $\partial_{c}\left(\mathbb{U}_{\mathrm{A}}^{\prime}\right)^{\varepsilon}$. Observe that (1.11.5) and section 2.3 yield an A-algebra isomorphism

$$
\mathbb{T}_{\mathrm{A}}^{0} \simeq \mathbb{E}_{\mathrm{A}} / / \overline{\bar{\chi}} \otimes \varepsilon\left(\iota\left(\mathbb{U}_{\pi, \mathrm{A}}^{\prime}\right) \otimes \mathbb{U}_{\mathrm{A}}^{\prime}\right)
$$

Hence the map $\phi$ factors through an A-linear map $\mathbb{T}_{\mathrm{A}}^{0} \rightarrow \mathbb{D}_{0, \mathrm{~A}, \star}^{\prime}$. Compare (1.11.10). Composing it with the conjugation by $\ell(\pi)$ we get an A-linear map

$$
\Phi_{\mathrm{A}}: \mathbb{T}_{\mathrm{A}}^{0} \rightarrow \mathbb{D}_{0, \mathrm{~A}, \star}^{\prime} \cdot
$$

Next, recall that we have proved in section 1.11 that $\Phi_{\mathcal{K}}$ factors to a $\mathcal{K}$-algebra homomorphism

$$
\Phi_{\mathcal{K}}: \mathbb{T}^{0} \rightarrow \mathbb{D}_{0, \star}^{\Sigma_{n}}
$$

Observe also the following easy fact.

2.8.2. Lemma. Let $A$ be a CID with fraction field $\mathrm{k}$. Let $\mathbf{A}, \mathbf{B}$ be A-algebras. Assume that $\mathbf{B}$ is a free A-module and that $\mathbf{B}^{\prime} \subset \mathbf{B}$ is a subalgebra which is a direct summand as an $\mathrm{A}$-module. Let $f: \mathbf{A} \rightarrow \mathbf{B}$ be an $\mathrm{A}$-linear map such that $f \otimes \mathrm{id}_{\mathrm{k}}$ 
factors through a k-algebra homomorphism $\mathbf{A} \otimes \mathrm{k} \rightarrow \mathbf{B}^{\prime} \otimes \mathrm{k}$. Then $f$ factors also through an A-algebra homomorphism $\mathbf{A} \rightarrow \mathbf{B}^{\prime}$.

Thus we have an $\mathcal{A}$-algebra homomorphism

$$
\Phi_{\mathcal{A}}: \mathbb{T}_{\mathcal{A}}^{0} \rightarrow \mathbb{D}_{0, \mathcal{A}, \star}^{\Sigma_{n}} .
$$

Therefore, we have the following commutative square:

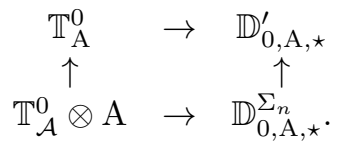

The lower map is an algebra homomorphism. Now, the proof consists of two steps.

Step 1. First, we set $(\mathcal{A}, \mathrm{A})=\left(\mathcal{A}_{b}, \mathrm{~A}_{b}\right)$ and we prove that $\Phi_{b}$ factors through an $\mathrm{A}_{b}$-algebra homomorphism

$$
\Phi_{b}: \mathfrak{T}\left(T_{b, \star}\right) \rightarrow \mathbf{D}_{0, b, \star}^{\Sigma_{n}} .
$$

Note that $\mathfrak{T}\left(T_{b, \star}\right)$ is a localization of $\mathbf{T}_{b}^{0}$; see section 2.7 and 3.4.1(b) below for details. By 2.8.2, (2.8.3) it is enough to prove that the map is obviously surjective:

$$
\mathbb{T}_{\mathcal{A}_{b}}^{0} \otimes \mathrm{k}_{b} \rightarrow \mathbf{T}_{\mathrm{k}_{b}}^{0} .
$$

The proof is the same as the proof of 2.7.3(b). We will only sketch it. By 2.3.6(b) it is enough to prove that the natural map below is surjective:

$$
f:\left(\mathbf{S}_{\mathrm{k}_{b}}^{\pi}\right)^{0} \rightarrow \mathbf{T}_{\mathrm{k}_{b}}^{0} .
$$

Note that the spectrum of $\mathcal{O}\left(T_{\pi, \mathrm{k}_{b}}\right)^{0}$ is a smooth symplectic $\mathrm{k}_{b}$-scheme by 4.1.1(c). Thus the cokernel of $f$ is a projective $\mathcal{O}\left(T_{\mathrm{k}_{b}}\right)$-module by A.10.3(a). Now, let A be the local ring of $\mathbb{Z}[\tau, \zeta] \subset \mathrm{k}_{b}$ at $\tau-1$. The residue field of $\mathrm{A}$ is $\mathrm{k}=\mathbb{F}_{p}(\zeta)$. Since $p$ is large enough the map

$$
\mathcal{O}\left(\bar{S}_{\pi, \mathrm{k}}\right)^{0} \rightarrow \mathcal{O}\left(T_{\pi, \mathrm{k}}\right)^{0}
$$

is surjective by A.11.3. Since $\tau=1 \mathrm{in} \mathrm{k}$, by 2.5.7( $(a)$ and 2.6.11(a) we have

$$
\mathbf{S}_{\mathrm{k}}^{\pi}=\mathcal{O}\left(\bar{S}_{\pi, \mathrm{k}}\right), \quad \mathbf{T}_{\mathrm{k}}=\mathcal{O}\left(T_{\pi, \mathrm{k}}\right) .
$$

So 2.3.6(b) yields

$$
\left(\mathbf{S}^{\pi}\right)^{0} \otimes \mathrm{k}=\mathcal{O}\left(\bar{S}_{\pi, \mathrm{k}}\right)^{0} .
$$

By A.11.2 the A-module $\mathbf{T}$ is flat. Compare 2.3.6(c). Thus 2.3.3(a) yields an inclusion

$$
\mathbf{T}^{0} \otimes \mathrm{k} \subset \mathcal{O}\left(T_{\pi, \mathrm{k}}\right)^{0} .
$$

Since the map (2.8.5) is surjective the map $f$ specializes to a surjective map:

$$
\left(\mathbf{S}^{\pi}\right)^{0} \otimes \mathrm{k} \rightarrow \mathbf{T}^{0} \otimes \mathrm{k} .
$$

Hence Nakayama's lemma implies that the cokernel of $f$ is an $\mathcal{O}\left(T_{\mathrm{k}_{b}}\right)$-module which is generically trivial. Since it is also projective, the map $f$ is surjective.

Step 2. Next, we take $\mathrm{A}=\mathbb{C}$. To unburden the notation we drop the subscript $\mathbb{C}$ at each place. Now, we prove that the map $\Phi$ factors through a $\mathbb{C}$-algebra homomorphism

$$
\Phi: \mathfrak{T}\left(T_{\star}\right) \rightarrow \mathbf{D}_{0, \star}^{\Sigma_{n}} .
$$


By (2.8.4) we have the commutative square

$$
\begin{array}{ccc}
\mathfrak{T}\left(T_{\star}\right) & \rightarrow & \mathbf{D}_{0, \star}^{\prime} \\
\uparrow & \uparrow \\
\mathfrak{T}\left(T_{b, \star}\right) \otimes \mathbb{C} & \rightarrow & \mathbf{D}_{0, \star}^{\Sigma_{n}},
\end{array}
$$

where the lower horizontal map is a $\mathbb{C}$-algebra homomorphism. By 2.7.3(b) the left vertical map is surjective. This implies the claim.

(b) By 2.7.1(a) there is a natural algebra homomorphism

$$
\mathcal{O}\left(T_{\star}\right) \rightarrow \mathfrak{T}\left(T_{\star}\right) .
$$

There is also an obvious inclusion $\mathcal{D}_{0, \star}^{\Sigma_{n}} \subset \mathbf{D}_{0, \star}^{\Sigma_{n}}$. We must prove that the map $\Phi$ in (2.8.6) takes $\mathcal{O}\left(T_{\star}\right)$ into $\mathcal{D}_{0, \star}^{\Sigma_{n}}$. For each monomial $u$ a computation yields

$$
\nabla\left(e_{i} u\right)=\left(\tau^{-\alpha_{i} \cdot|u|-2} \otimes q^{-\alpha_{i}}\right) \nabla\left(u e_{i}\right)+\left(e_{i} \otimes 1\right) \nabla(u) .
$$

An easy induction using (2.8.7) yields elements $a_{m^{\prime}, m} \in \mathbf{U} \otimes \mathbf{D}_{0, \star}$ such that $1-a_{m, m}$ is invertible and we have

$$
\nabla\left(\dot{e}^{m} u\right)=\sum_{\left|m^{\prime}\right| \leqslant|m|} a_{m, m^{\prime}} \nabla\left(u \dot{e}^{m^{\prime}}\right) .
$$

Therefore, if $u \in Z(\mathbf{U})$ there are elements $b_{m^{\prime}, m} \in \mathbf{U} \otimes \mathbf{D}_{0, \star}$ such that

$$
\nabla\left(\dot{e}^{m} u\right)=\sum_{\left|m^{\prime}\right|<|m|} b_{m, m^{\prime}} \nabla\left(\dot{e}^{m^{\prime}} u\right) .
$$

A similar identity holds for $\nabla\left(\iota\left(\dot{f}^{n}\right) u\right)$. So for each $u \in Z(\mathbf{U}), \lambda \in X$ and each tuple $m, n$ of integers $\geqslant 0$ we have

$$
\nabla\left(\dot{e}^{m} u\right)=\nabla\left(\dot{e}^{m}\right) \nabla(u), \quad \nabla\left(\iota\left(\dot{f}^{n}\right) u\right)=\nabla\left(\iota\left(\dot{f}^{n}\right)\right) \nabla(u), \quad \nabla\left(z_{\lambda}\right)=\partial_{\triangleright}\left(z_{\lambda}\right) \otimes 1 .
$$

Thus the restriction of the map $\nabla$ to $\mathcal{U}$ is an algebra homomorphism. For each positive root $\alpha$ there are constants $c_{m, m^{\prime}} \in \mathcal{A}$ such that

$$
\Delta\left(\dot{e}_{\alpha}^{l}\right)=k_{-l \alpha} \otimes e_{\alpha}^{l}+\sum_{m, m^{\prime}} c_{m, m^{\prime}} k_{-l\left|m^{\prime}\right|} \dot{e}^{l m} \otimes \dot{e}^{l m^{\prime}} \text { modulo }(q-\tau) \mathbb{U}_{\mathcal{A}}^{\otimes 2} .
$$

Here $m, m^{\prime}$ are tuples of integers $\geqslant 0$ such that $0 \neq|m|$ and $|m|+\left|m^{\prime}\right|=\alpha$. See also (2.2.1). So we have

$$
\left(1-1 \otimes q^{-l \alpha}\right) \nabla\left(x_{\alpha}\right)=\sum_{m, m^{\prime}} c_{m, m^{\prime}}\left(\dot{e}^{l m} \otimes q^{-l\left|m^{\prime}\right|}\right) \nabla\left(\dot{e}^{l m^{\prime}}\right) .
$$

Further, (2.8.7) implies that

$$
\nabla\left(x_{i}\right)=\sum_{j \geqslant 0} x_{i} \otimes q^{-j l \alpha_{i}} \in \mathcal{U} \otimes \mathcal{D}_{0, \star} .
$$

So an induction on the positive integer $\rho \cdot \alpha$ shows that $\nabla\left(x_{\alpha}\right) \in \mathcal{U} \otimes \mathcal{D}_{0, \star}$ for each $\alpha$. The same argument works also for $\nabla\left(y_{\alpha}\right)$, yielding the inclusion

$$
\nabla(\mathcal{U}) \subset \mathcal{U} \otimes \mathcal{D}_{0, \star}
$$

Hence we have

$$
\Phi\left(\mathcal{O}\left(T_{\star}\right)\right) \subset \mathcal{D}_{0, \star}^{\Sigma_{n}} .
$$

Finally, we must prove that the algebra homomorphism

$$
\Phi: \mathcal{O}\left(T_{\star}\right) \rightarrow \mathcal{D}_{0, \star}^{\Sigma_{n}}
$$


is indeed a Poisson algebra homomorphism. Note that $\mathbb{C}=\mathcal{A} /(q-\tau)$ with $\mathcal{A}=$ $\mathbb{C}\left[q^{ \pm 1}\right]$. The Poisson bracket on $\mathcal{O}\left(\bar{S}_{\pi}\right)^{0}$ is computed by lifting elements into $\mathbb{S}_{\mathcal{A}}^{\pi, 0}$ and computing their commutator there; see the proof of 2.5.6(e). Composing the $\mathcal{A}$-algebra homomorphism

$$
\Phi_{\mathcal{A}}: \mathbb{T}_{\mathcal{A}}^{0} \rightarrow \mathbb{D}_{0, \mathcal{A}, \star}^{\Sigma_{n}}
$$

with the natural map $\mathbb{S}_{\mathcal{A}}^{\pi, 0} \rightarrow \mathbb{T}_{\mathcal{A}}^{0}$ we get an algebra homomorphism $\mathbb{S}_{\mathcal{A}}^{\pi, 0} \rightarrow \mathbb{D}_{0, \mathcal{A}, \star}^{\Sigma_{n}}$. It factors to a Poisson algebra homomorphism

$$
\mathcal{O}\left(\bar{S}_{\pi}\right)^{0} \rightarrow \mathcal{D}_{0, \star}^{\Sigma_{n}}
$$

by the discussion above. The theorem follows, because the map

$$
\mathcal{O}\left(\bar{S}_{\pi}\right)^{0} \rightarrow \mathcal{O}(N)
$$

is a surjective Poisson algebra homomorphism. See the proof of 2.6.2(b) for details.

\section{The double affine Hecke Algebra}

3.1. Global dimension. Let $(\mathcal{A}, \mathrm{A})$ be a modular pair with $\mathcal{A}=\mathbb{Z}\left[q^{ \pm 1}, t^{ \pm 1}\right]$. The DAHA is the $\mathcal{A}$-algebra $\mathbb{H}=\mathbb{H}_{\mathcal{A}}$ generated by $t_{\hat{w}}, x_{\lambda}, t_{\pi}$ with $\hat{w} \in \widehat{\Sigma}_{n}, \lambda \in \widetilde{X}$, $\pi \in P$ modulo the defining relations

$$
\begin{gathered}
\left(t_{s_{i}}-t\right)\left(t_{s_{i}}+t^{-1}\right)=0, \quad t_{s_{i}} x_{\lambda}-x_{s_{i} \lambda} t_{s_{i}}=\left(t-t^{-1}\right)\left(x_{\lambda}-x_{s_{i} \lambda}\right) /\left(1-x_{\alpha_{i}}\right), \\
t_{\hat{w}} t_{\hat{w}^{\prime}}=t_{\hat{w} \hat{w}^{\prime}} \text { if } \ell\left(\hat{w} \hat{w}^{\prime}\right)=\ell(\hat{w})+\ell\left(\hat{w}^{\prime}\right), \quad t_{\pi} t_{s_{i}} t_{\pi}^{-1}=t_{\pi\left(s_{i}\right)}, \\
x_{\lambda} x_{\mu}=x_{\lambda+\mu}, \quad t_{\pi} x_{\lambda} t_{\pi}^{-1}=x_{\pi(\lambda)}, \quad x_{\delta}=q^{-2}, \quad x_{\alpha_{0}}=q^{-2} x_{-\theta} .
\end{gathered}
$$

The subalgebra $\mathbb{H}^{y}$ generated by $\left\{t_{\hat{w}}, t_{\pi}\right\}$ is the Iwahori-Hecke algebra of the extended affine Weyl group $\widetilde{\Sigma}_{n}$. It may also be viewed as the algebra generated by elements $t_{\tilde{w}}$ with $\tilde{w} \in \widetilde{\Sigma}_{n}$ modulo well-known relations.

Set $y_{\lambda-\lambda^{\prime}}=t_{\tau_{\lambda}}\left(t_{\tau_{\lambda^{\prime}}}\right)^{-1}$ for each $\lambda, \lambda^{\prime} \in X_{+}$. The multiplication in $\mathbb{H}$ yields a linear isomorphism

$$
\mathbb{X} \otimes \mathbb{H}^{y} \simeq \mathbb{X} \otimes \mathbb{H}_{0} \otimes \mathbb{Y} \simeq \mathbb{Y} \otimes \mathbb{H}_{0} \otimes \mathbb{X} \simeq \mathbb{H}
$$

where $\mathbb{X}, \mathbb{Y}$ and $\mathbb{H}_{0}$ are the subalgebras generated by $\left\{x_{\lambda} ; \lambda \in X\right\},\left\{y_{\lambda} ; \lambda \in X\right\}$ and $\left\{t_{w} ; w \in \Sigma_{n}\right\}$, respectively. We set $t_{i}=t_{s_{i}}, x_{i}=x_{\varepsilon_{i}}$ and $y_{i}=y_{\varepsilon_{i}}$. The Cherednik-Fourier transform is the automorphism $\mathfrak{F}_{H}: \mathbb{H} \rightarrow \mathbb{H}$ given by

$$
x_{i}, y_{i}, t_{i}, q, t \mapsto y_{i}, x_{i}, t_{i}^{-1}, q^{-1}, t^{-1} .
$$

Write $\mathbb{H}_{\mathrm{A}}=\mathbb{H} \otimes \mathrm{A}$. Recall that $\tau, \zeta$ are the images of $q, t$ in A. We call $\tau$ the modular parameter and $\zeta$ the quantum parameter. The SDAHA (spherical subalgebra) is

$$
\mathbb{S H}_{\mathrm{A}}=\left\{x \in \mathbb{H}_{\S} ; t_{i} x=x t_{i}=t x, \forall i\right\} .
$$

Set $o=\sum_{w} t^{\ell(w)} t_{w}$. Let $a_{o} \in \mathrm{A}$ be such that $o^{2}=a_{o} o$. If $a_{o}$ is invertible, then we have $\mathbb{S H}_{\mathrm{A}}=o \mathbb{H}_{\mathrm{A}} o$. So $\mathbb{S H}_{\mathrm{A}}$ is a direct summand of $\mathbb{H}_{\mathrm{A}}$ as an A-module. Hence it is a free A-module. Note that $\mathbb{S H}_{\mathrm{A}}$ is a $\mathbb{X}_{\mathrm{A}}^{\Sigma_{n}} \otimes \mathbb{Y}_{\mathrm{A}}^{\Sigma_{n}}$-module of finite type, with $\mathbb{X}_{\mathrm{A}}^{\Sigma_{n}}$ acting by left multiplication and $\mathbb{Y}_{\mathrm{A}}^{\Sigma_{n}}$ by right multiplication

3.1.2. Theorem. Assume that $\mathrm{A}$ is a field and that $a_{o} \neq 0$. The ring $\mathbb{H}_{\mathrm{A}}$ has a finite left and right global dimension. 
Proof. Let $\mathbb{X}_{\mathrm{A}}^{+} \subset \mathbb{X}_{\mathrm{A}}$ be the subalgebra generated by $\left\{x_{i}\right\}$. Let $\mathbb{H}_{\mathrm{A}}^{+} \subset \mathbb{H}_{\mathrm{A}}$ be the subalgebra generated by $\mathbb{H}_{0, \mathrm{~A}}, \mathbb{Y}_{\mathrm{A}}$ and $\mathbb{X}_{\mathrm{A}}^{+}$. The multiplication yields a linear isomorphism from $\mathbb{X}_{\mathrm{A}}^{+} \otimes \mathbb{H}_{\mathrm{A}}^{y} \rightarrow \mathbb{H}_{\mathrm{A}}^{+}$. Equip $\mathbb{H}_{\mathrm{A}}^{+}$with the $\mathbb{Z}_{+}$-grading such that

$$
\operatorname{deg}\left(x_{\lambda}\right)=1, \operatorname{deg}\left(t_{\tilde{w}}\right)=0, \forall \lambda \in X, \tilde{w} \in \widetilde{\Sigma}_{n} .
$$

Let us prove that $\mathbb{H}_{\mathrm{A}}$ has a finite left global dimension. Consider the 2-sided ideal $I=\sum_{i} x_{i} \mathbb{H}_{\mathrm{A}}^{+}$of $\mathbb{H}_{\mathrm{A}}^{+}$. The following properties are immediate from (3.1.1):

- for all $i, j$ we have $x_{i} x_{j}=x_{j} x_{i}$,

- for all $h \in \mathbb{H}_{\mathrm{A}}^{+}$and all $j$ such that $x_{j+1} h \in \sum_{i=1}^{j} x_{i} \mathbb{H}_{\mathrm{A}}^{+}$we have $h \in$ $\sum_{i=1}^{j} x_{i} \mathbb{H}_{\mathrm{A}}^{+}$,

- we have $I=\sum_{i} x_{i} \mathbb{H}_{\mathrm{A}}^{+}=\sum_{i} \mathbb{H}_{\mathrm{A}}^{+} x_{i}$, a proper ideal of $\mathbb{H}_{\mathrm{A}}^{+}$.

Therefore [MR, thm. 7.3.16] yields

$$
\operatorname{pd}_{\mathbb{H}_{\mathrm{A}}^{+}}\left(\mathbb{H}_{\mathrm{A}}^{+} / I\right)=n .
$$

Recall that $\mathrm{fd}_{\mathbb{H}_{\mathrm{A}}^{+}}\left(\mathbb{H}_{\mathrm{A}}^{+} / I\right) \leqslant \operatorname{pd}_{\mathbb{H}_{\mathrm{A}}^{+}}\left(\mathbb{H}_{\mathrm{A}}^{+} / I\right)$; see $[\mathrm{MR}$, sec. 7.1.5]. Thus for any finitely generated graded left $\mathbb{H}_{\mathrm{A}}^{+}$-module $M$ we have $[\mathrm{MR}$, thm. 7.3.14]

$$
\operatorname{pd}_{\mathbb{H}_{\mathrm{A}}^{+}}(M) \leqslant \operatorname{lgd}\left(\mathbb{H}_{\mathrm{A}}^{+} / I\right)+n .
$$

Note that $I$ is not contained in the Jacobson radical of $\mathbb{H}_{\mathrm{A}}^{+}$. However, in the proof of [op. cit.] this hypothesis is used only in deducing that $M=0$ from the equation $M I=M$. This implication holds in our case because $M$ is graded and $I$ has positive degrees. Note also that to compute $\operatorname{lgd}\left(\mathbb{H}_{\mathrm{A}}^{+}\right)$it is enough to consider only finite graded left modules. See [MR, sec. 7.1.8] and [NV1, sec. I.7.8]. Thus we have proved that

$$
\operatorname{lgd}\left(\mathbb{H}_{\mathrm{A}}^{+}\right) \leq \operatorname{lgd}\left(\mathbb{H}_{\mathrm{A}}^{+} / I\right)+n
$$

Next, since $\mathbb{H}_{\mathrm{A}}$ is the localization of $\mathbb{H}_{\mathrm{A}}^{+}$at $x_{\omega_{n}}$ we also have

$$
\operatorname{lgd}\left(\mathbb{H}_{\mathrm{A}}\right) \leqslant \operatorname{lgd}\left(\mathbb{H}_{\mathrm{A}}^{+} / I\right)+n
$$

by $\left[\mathrm{MR}\right.$, cor. 7.4.3]. Now $\mathbb{H}_{\mathrm{A}}^{+} / I \simeq \mathbb{H}_{\mathrm{A}}^{y}$ as an A-algebra. Thus we have

$$
\operatorname{lgd}\left(\mathbb{H}_{\mathrm{A}}\right) \leqslant \operatorname{lgd}\left(\mathbb{H}_{\mathrm{A}}^{y}\right)+n .
$$

Arguing as above with $\mathbb{H}_{\mathrm{A}}$ replaced by $\mathbb{H}_{\mathrm{A}}^{y}$ and $x_{i}$ by $y_{i}$ we also get that

$$
\operatorname{lgd}\left(\mathbb{H}_{\mathrm{A}}\right) \leqslant \operatorname{lgd}\left(\mathbb{H}_{0, \mathrm{~A}}\right)+2 n .
$$

Finally, $\mathbb{H}_{0, \mathrm{~A}}$ is the Iwahori-Hecke algebra of type $G L$. It is well known that it has a finite global dimension iff $a_{o} \neq 0$.

3.2. Roots of unity. Unless specified otherwise we will always assume that $a_{o}$ is invertible. We will be interested by the representations of $\mathbb{H}_{\mathrm{A}}$ for $\mathrm{A}=\mathbb{C}$ and $\tau, \zeta$ both roots of unity. Let $l$ be the order of $\tau$. If it is finite we will always assume that $l=p^{e}$, an odd prime power. As before, if $l$ is finite we write

$$
\mathbf{S H}=\mathbb{S H}_{\mathrm{A}}, \quad \mathbf{H}=\mathbb{H}_{\mathrm{A}} .
$$

Let $\left(\mathcal{A}_{b}, \mathrm{~A}_{b}, \mathrm{k}_{b}\right)$ be as in 2.2.5(a). We will abbreviate $\mathrm{k}_{b}=\mathbb{C}$, hoping it will not create any confusion.

Fix integers $m<0<k$ such that $(k, m)=1$ and $(\tau, \zeta) \in \Gamma_{c}(\mathbb{C})$. Obviously, the choice of $(k, m)$ is not unique. We adopt the following strategy: first we fix $c$, then we choose $(\tau, \zeta) \in \Gamma_{c}(\mathbb{C})$ such that $\tau, \zeta$ are roots of unity and $p$ is large enough; finally we prove our main result for these parameters. 
Let us briefly explain what the restrictions on $c$ are. Its sign is indifferent, because there is an involution

$$
\mathrm{IM}: \mathbb{H} \rightarrow \mathbb{H}, t_{i} \mapsto-t^{2} t_{i}^{-1}, x_{\lambda} \mapsto x_{-\lambda}, q \mapsto q^{-1}, t \mapsto t .
$$

For 3.6.9 to hold we will assume that $c<-2$ and for 3.5.1 to hold that $k>2 n$. Finally, in 4.2.1(b) we will choose $m=-1$, i.e., we will set $\zeta^{l}=1$.

3.3. The canonical embedding. Assume that $a_{o}$ is invertible. The subscript $\star$ will denote a quotient ring as in section 1.11. There is a unique algebra homomorphism

given by

$$
\Psi^{\prime}: \mathbb{H}_{\mathrm{A}} \rightarrow \mathbb{D}_{0, \mathrm{~A}, \star} \rtimes \Sigma_{n}
$$

$$
t_{i} \mapsto \zeta s_{i}+\left(\zeta-\zeta^{-1}\right)\left(1-q^{\alpha_{i}}\right)^{-1}\left(1-s_{i}\right), \quad x_{\lambda} \mapsto q^{\lambda}, \quad t_{\pi} \mapsto \pi
$$

Here we have set $q^{\delta}=\tau^{-2}$. The inclusion of $\widetilde{\Sigma}_{n}$ into the rhs is given by

$$
w \mapsto(0, w), \quad \tau_{\lambda} \mapsto\left(k_{-2 \lambda}, 1\right) .
$$

Since $\mathbb{D}_{0, A, \star}$ is an ID there is a unique algebra homomorphism

$$
\Psi: \mathbb{S H}_{\mathrm{A}} \rightarrow \mathbb{D}_{0, \mathrm{~A}, \star}^{\Sigma_{n}}
$$

such that $\Psi^{\prime}(x o)=\Psi(x) \Psi^{\prime}(o)$. The following is standard; see [C, 3.2.1].

3.3.1. Proposition. The maps $\Psi, \Psi^{\prime}$ are injective and yield algebra isomorphisms

$$
\Psi: \mathbb{S H}_{\mathrm{A}, \star} \rightarrow \mathbb{D}_{0, \mathrm{~A}, \star}^{\Sigma_{n}}, \quad \Psi^{\prime}: \mathbb{H}_{\mathrm{A}, \star} \rightarrow \mathbb{D}_{0, \mathrm{~A}, \star} \rtimes \Sigma_{n} .
$$

3.4. The center of SDAHA. Assume that $a_{o}$ is invertible and that $l$ is finite. In this section we recall a few basic facts on the center of $\mathbf{S H}$. Let $\mathcal{X}, \mathcal{Y} \subset \mathbf{H}$ be the subalgebras generated respectively by $\left\{x_{\lambda} ; \lambda \in l X\right\},\left\{y_{\lambda} ; \lambda \in l X\right\}$. We put

$$
\mathcal{L}=\mathcal{X}^{\Sigma_{n}} \otimes \mathcal{Y}^{\Sigma_{n}}
$$

Set $\sigma_{x}=\prod_{\alpha, k}\left(q^{l \alpha}-\zeta^{2 l k}\right)$ where $\alpha$ runs over $\Pi$ and $k=0,1$. We view $\sigma_{x}$ as an element of $\mathcal{L}$ as in (3.4.2). Put $\sigma_{y}=\mathfrak{F}_{H}\left(\sigma_{x}\right)$.

3.4.1. Proposition. The following hold:

(a) $\mathcal{L} \subset Z(\mathbf{H}), o \mathcal{L} \subset Z(\mathbf{S H})$ and $\mathbf{H}, \mathbf{S H}$ are $\mathcal{L}$-modules of finite type,

(b) $\mathbf{D}_{0, \star}^{\Sigma_{n}}, \mathbf{T}^{0}$ are $\mathcal{L}$-algebras and $\mathfrak{T}\left(T_{\star}\right)$ is the localization of $\mathbf{T}^{0}$ at $\sigma_{x}$,

(c) the maps $\Phi: \mathfrak{T}\left(T_{\star}\right) \rightarrow \mathbf{D}_{0, \star}^{\Sigma_{n}}$ and $\Psi: \mathbf{S H}_{\star} \rightarrow \mathbf{D}_{0, \star}^{\Sigma_{n}}$ are $\mathcal{L}$-linear.

Proof. (a) This is well known.

(b), (c) There are algebra isomorphisms

$$
\begin{aligned}
& \mathbf{X} \rightarrow \mathbf{F}_{0}, \quad x_{\lambda} \mapsto q^{\lambda}, \\
& \mathbf{Y} \rightarrow \mathbf{U}_{0}, \quad y_{\lambda} \mapsto k_{\lambda} .
\end{aligned}
$$

Thus $\varrho_{0}, \Omega$ yield algebra isomorphisms

$$
\begin{aligned}
& \mathbf{X}^{\Sigma_{n}} \simeq Z\left(\mathbf{F}^{\prime}\right) \simeq Z\left(\mathbb{F}_{\mathcal{A}}^{\prime}\right) \otimes \mathrm{A}, \\
& \mathbf{Y}^{\Sigma_{n}} \simeq Z\left(\mathbf{U}^{\prime}\right) \simeq Z\left(\mathbb{U}_{\mathcal{A}}^{\prime}\right) \otimes \mathrm{A} .
\end{aligned}
$$

Therefore the maps (1.11.2)

$$
\begin{aligned}
& z^{\prime}: Z\left(\mathbb{F}_{\mathcal{A}}^{\prime}\right) \rightarrow \mathbb{T}_{\mathcal{A}}^{0}, \\
& z: Z\left(\mathbb{U}_{\mathcal{A}}^{\prime}\right) \rightarrow \mathbb{T}_{\mathcal{A}}^{0}
\end{aligned}
$$


yield an algebra homomorphism $\mathcal{L} \rightarrow \mathbf{T}^{0}$. Now, the maps (1.11.3)

$$
\begin{aligned}
& L^{\prime}: Z\left(\mathbb{F}_{\mathcal{A}}^{\prime}\right) \rightarrow \mathbb{D}_{0, \mathcal{A}, \star}^{\Sigma_{n}}, \\
& L: Z\left(\mathbb{U}_{\mathcal{A}}^{\prime}\right) \rightarrow \mathbb{D}_{0, \mathcal{A}, \star}^{\Sigma_{n}}
\end{aligned}
$$

yield an algebra homomorphism $\mathcal{L} \rightarrow \mathbf{D}_{0, \star}^{\Sigma_{n}}$. The map $\Phi$ is $\mathcal{L}$-linear by 1.11.4.

The $\mathcal{L}$-algebra structure on $\mathbf{S H}$ is given by

$$
f \mapsto o f, \quad u \mapsto o u, \quad \forall f \in \mathcal{X}^{\Sigma_{n}}, u \in \mathcal{Y}^{\Sigma_{n}} .
$$

It is well known that

$$
\Psi(o f)=L^{\prime}(f), \quad \Psi(o u)=L(u), \quad \forall f, u ;
$$

see [C], [K2, Theorem 5.9]. So the map $\Psi$ is $\mathcal{L}$-linear.

3.4.3. Proposition. Assume that $\mathrm{A}=\mathbb{C}$. We have that $\mathbf{S H}$ is a $Z(\mathbf{S H})$-module of finite type. It is a NID and a maximal order. Further, $Z(\mathbf{S H})$ is a direct summand of $\mathbf{S H}$ as a $Z(\mathbf{S H})$-module.

Proof. Recall that $\mathbf{S H}_{\sigma_{x}}$ is the localization of $\mathbf{S H}$ at $\sigma_{x}$. By 3.3.1 we have $\mathbf{S H}_{\sigma_{x}} \simeq$ $\mathbf{D}_{0, \star}^{\Sigma_{n}}$. Thus $\mathbf{S H}_{\sigma_{x}}$ is an ID. Note that $\sigma_{x}$ is a regular element in $\mathbf{S H}$ by (3.1.1). Thus $\mathbf{S H} \subset \mathbf{S H}_{\sigma_{x}}$. Thus $\mathbf{S H}$ is an ID. Since $\mathbf{S H}$ is a $Z(\mathbf{S H})$-module of finite type (see 3.4.1(b)), it is an order in its quotient ring by Posner's theorem, see section A.10.

Let us prove that $\mathbf{S H}$ is maximal in its equivalence class. Fix a ring $\mathbf{A}$ and an element $0 \neq z \in Z(\mathbf{S H})$ such that

$$
\mathbf{S H} \subset \mathbf{A} \subset z^{-1} \mathbf{S H} .
$$

For future use, note that $\mathbf{A}$ is a torsion-free $\mathcal{L}$-module of finite type; see 3.4.6(b) below. Observe that $\mathbf{D}_{0}$ is an Azumaya algebra. Hence $\mathbf{D}_{0, \star}$ is as well. Thus $\mathbf{D}_{0, \star}^{\Sigma_{n}}$ is again an Azumaya algebra by faithfully-flat descent, because the group $\Sigma_{n}$ acts freely. Therefore the quotient rings $\mathbf{S H}_{\sigma_{x}}, \mathbf{S H}_{\sigma_{y}}$ are both maximal orders. Thus we have $\mathbf{A}_{\sigma_{x}}=\mathbf{S H}_{\sigma_{x}}$ and $\mathbf{A}_{\sigma_{y}}=\mathbf{S H}_{\sigma_{y}}$. The elements $\sigma_{x}, \sigma_{y}$ are regular in $\mathbf{H}$ by (3.1.1). Hence they are also regular in $\mathbf{A}$. So we have

$$
\mathbf{A} \subset \mathbf{S H}_{\sigma_{x}} \cap \mathbf{S H}_{\sigma_{y}} .
$$

Thus the $\mathcal{L}$-module $\mathbf{A} / \mathbf{S H}$ is supported on a closed subset of $\operatorname{Spec}(\mathcal{L})$ of codimension $\geqslant 2$. Since the $\mathcal{L}$-module $\mathbf{S H}$ is free we have $\mathbf{A}=\mathbf{S H}$ by 3.4.4. The second claim is a direct consequence of the first one. See section A.10.

3.4.4. Lemma. Let A be a commutative affine ring, $M$ a torsion-free A-module of finite type and $P \subset M$ a projective A-submodule such that $M / P$ is supported on a closed subset of $\mathrm{Spec}(\mathrm{A})$ of codimension $\geqslant 2$. Then $P=M$.

3.4.5. Proposition. Assume that $\mathrm{A}=\mathbb{C}$. The scheme $C M=\operatorname{Spec}(Z(\mathbf{S H}))$ is integral, normal and Cohen-Macaulay.

Proof. The $\operatorname{ring} Z(\mathbf{S H})$ is a CID by 3.4.3. Thus $C M$ is an integral scheme. The $\mathcal{L}$-module $Z(\mathbf{S H})$ is projective and of finite type by 3.4.3. Thus it is a free $\mathcal{L}$-module by the Pittie-Steinberg and Quillen-Suslin theorems. So the variety $C M$ is CohenMacaulay, because $\operatorname{Spec}(\mathcal{L})$ is smooth. An irreducible Cohen-Macaulay variety which is smooth away from codimension $\geqslant 2$ closed subset is normal. Further, 
$\operatorname{Spec}\left(Z(\mathbf{S H})_{\sigma_{x}}\right)$ is smooth, because $\mathbf{S H}_{\sigma_{x}} \simeq \mathbf{D}_{0, \star}^{\Sigma_{n}}$. Hence $\operatorname{Spec}\left(Z(\mathbf{S H})_{\sigma_{y}}\right)$ is also smooth. The complement of

$$
\operatorname{Spec}\left(Z(\mathbf{S H})_{\sigma_{x}}\right) \cup \operatorname{Spec}\left(Z(\mathbf{S H})_{\sigma_{y}}\right)
$$

in $C M$ is a closed subset of codimension $\geqslant 2$, because $Z(\mathbf{S H})$ is an $\mathcal{L}$-module of finite type and the closed subset

$$
\left\{\sigma_{x}=\sigma_{y}=0\right\} \subset \operatorname{Spec}(\mathcal{L})
$$

has codimension $\geqslant 2$. Thus the variety $C M$ is normal.

\subsubsection{Remarks.}

(a) The rings $Z(\mathbf{H}), Z(\mathbf{S H})$ are isomorphic. See section A.14 for details.

(b) By (3.1.1) and the Pittie-Steinberg theorem the $\mathcal{L}$-module $\mathbf{H}$ is free and of finite type. Since $\mathbf{S H}$ is a direct summand of $\mathbf{H}$, it is also a free $\mathcal{L}$-module of finite type by the Quillen-Suslin theorem.

3.5. From SDAHA to QDO. Set $\mathrm{A}=\mathbb{C}$. Fix $c \in \mathbb{Q}^{\times}$. Assume that $a_{o}$ is invertible. Recall the maps

$$
\Psi: \mathbf{S H} \rightarrow \mathbf{D}_{0, \star}^{\Sigma_{n}}, \quad \Phi: \mathbf{T}^{0} \rightarrow \mathbf{D}_{0, \star}^{\Sigma_{n}}
$$

given in 3.3.1 and section 2.8. In 4.2.1(b) we compare the algebras $\mathbf{S H}$ and $\mathfrak{T}(T)$, where $\mathfrak{T}$ is the sheaf of algebras over the deformed Hilbert scheme $T$ introduced in section 2.7. A first step towards this is the following inclusion. The proof is technical. It is given in section A.15.

3.5.1. Proposition. Let $(\tau, \zeta) \in \Gamma_{c}(\mathbb{C})$. If $k>2 n$ and $l$ is finite and large enough, then we have $\Psi(\mathbf{S H}) \subset \Phi\left(\mathbf{T}^{0}\right)$.

Note that the lower bound for $l$ in 3.5.1 may depend on $c$.

3.6. From SDAHA to DAHA. In this section we compare the categories $\operatorname{Mod}\left(\mathbb{H}_{A}\right)$ and $\mathcal{M o d}\left(\mathbb{S H}_{A}\right)$. First, let us recall the following standard result. See section A.16 for details.

3.6.1. Lemma. Let $\mathbf{A}$ be a ring and let $o \in \mathbf{A}$ be an idempotent. The full subcategory $\operatorname{Mod}(\mathbf{A}, o) \subset \mathcal{M o d}(\mathbf{A})$ of the modules killed by o is a Serre category. Further, there is an equivalence

$$
\operatorname{Mod}(\mathbf{A}) / \operatorname{Mod}(\mathbf{A}, o) \rightarrow \operatorname{Mod}(o \mathbf{A} o), \quad M \mapsto o M .
$$

Now, fix $c \in \mathbb{Q}^{\times}$. Assume that $\mathrm{A}=\mathbb{C}$ and that $a_{o}$ is invertible. To simplify the notation we will abbreviate $\mathbb{H}=\mathbb{H}_{\mathbb{C}}, \mathbb{X}=\mathbb{X}_{\mathbb{C}}$, etc. Consider the full subcategories

$$
\operatorname{Mod}(\mathbb{H})^{\prime} \subset \mathcal{M o d}(\mathbb{H}), \quad \mathcal{M o d}(\mathbb{S H})^{\prime} \subset \mathcal{M o d}(\mathbb{S H})
$$

consisting of the modules with a locally finite action of $\mathbb{X}, \mathbb{X}^{\Sigma_{n}}$. By 3.6.1 we have a quotient functor

$$
F^{*}: \operatorname{Mod}(\mathbb{H})^{\prime} \rightarrow \operatorname{Mod}(\mathbb{S H})^{\prime}, M \mapsto o M .
$$

3.6.2. Proposition. Let $(\tau, \zeta) \in \Gamma_{c}(\mathbb{C})$ and $\mathrm{A}=\mathbb{C}$. If $\tau, \zeta$ are not roots of unity and $c<-2$, then $F^{*}$ is an equivalence of categories. 
Proof. To unburden the notation we may use the same symbol for a $\mathbb{C}$-scheme and its set of closed $\mathbb{C}$-points. Consider the $\widetilde{\Sigma}_{n}$-action on $H=\mathbb{C}^{\times} \otimes_{\mathbb{Z}} X$ given by

$$
\tau_{\lambda} w(z \otimes \mu)=(z \otimes w \mu)(\tau \otimes \lambda), \quad \forall \lambda, \mu \in X, w \in \Sigma_{n}, z \in \mathbb{C}^{\times} .
$$

For each $\widetilde{\Sigma}_{n}$-orbit in $\omega \subset H$, consider the subcategories

$$
\operatorname{Mod}(\mathbb{H})^{\omega} \subset \mathcal{M o d}(\mathbb{H}), \quad \boldsymbol{M o d}(\mathbb{S H})^{\omega} \subset \mathcal{M o d}(\mathbb{S H})
$$

consisting of the modules whose support, as $\mathbb{X}^{\Sigma_{n}}$-modules, is contained in $\omega / \Sigma_{n}$. Thus we have

$$
\operatorname{Mod}(\mathbb{H})^{\prime}=\bigoplus_{\omega} \operatorname{Mod}(\mathbb{H})^{\omega}, \quad \operatorname{Mod}(\mathbb{S H})^{\prime}=\bigoplus_{\omega} \operatorname{Mod}(\mathbb{S H})^{\omega}
$$

The functor $F^{*}$ preserves this decomposition. Further, $\operatorname{Mod}(\mathbb{H})^{\omega}$ contains finitely many isomorphism classes of simple objects. Thus the category $\operatorname{Mod}(\mathbb{S H I})^{\omega}$ also contains finitely many isomorphism classes of simple objects. Let $d_{\omega}(\mathbb{H}), d_{\omega}(\mathbb{S H})$ be the those numbers. We have

$$
d_{\omega}(\mathbb{H}) \geqslant d_{\omega}(\mathbb{S H}) .
$$

We must prove that it is an equality.

The simple $\mathbb{H}$-modules are classified in $[\mathrm{V}]$ for each simple, connected and simply connected linear group. The simple modules of the double affine Hecke algebras of types $S L, G L$ are essentially the same; see [V] sec. 8], VV3, sec. 2.5]. Thus, to simplify the exposition, in the rest of this proof we will assume that $\mathbb{H}$ is the DAHA of type $S L$. In particular, the symbols $H, X$ will now denote the group of diagonal matrices in $S L$ and its group of cocharacters.

Now, we set

$$
\mathrm{F}=\mathbb{C}((\varepsilon)), \quad \mathrm{R}=\mathbb{C}[[\varepsilon]] .
$$

Let $\widetilde{S L}(\mathrm{~F})$ be the central extension of $S L(\mathrm{~F})$ by $\mathbb{C}^{\times}$as in $\left[\mathrm{V}\right.$, sec. 2.2]. Let $\widetilde{\mathfrak{s l}}_{\mathrm{F}}$ be the Lie algebra of $\widetilde{S L}(\mathrm{~F})$ and $\widetilde{H} \subset \widetilde{S L}(\mathrm{~F})$ the pullback of $H$ by the obvious projection $\widetilde{S L}(\mathrm{~F}) \rightarrow S L(\mathrm{~F})$. So there is an obvious map

$$
\tilde{H} \times \mathbb{C}^{\times} \rightarrow H \times \mathbb{C}^{\times} .
$$

Fix $s \in H$. Fix a point of $\tilde{H} \times \mathbb{C}^{\times}$over $(s, \zeta)$. We will denote this point by $(s, \zeta)$ again, hoping it will not create any confusion. Consider the automorphisms

$$
\begin{aligned}
& a_{s, \tau, \zeta}: \widetilde{S L}(\mathrm{~F}) \rightarrow \widetilde{S L}(\mathrm{~F}), \quad g(\varepsilon) \mapsto(\operatorname{ad} s)(g(\tau \varepsilon)), \\
& a_{s, \tau, \zeta}: \mathfrak{s l}_{\mathrm{F}} \rightarrow \mathfrak{s l}_{\mathrm{F}}, \quad x(\varepsilon) \mapsto \zeta^{-1}(\operatorname{ad} s)(x(\tau \varepsilon)) .
\end{aligned}
$$

We will abbreviate $a=a_{s, \tau, \zeta}$.

Let $\mathcal{N} \subset \mathfrak{s l}_{\mathrm{F}}$ be the nilpotent cone. Let $\widetilde{S L}(\mathrm{~F})^{a} \subset \widetilde{S L}(\mathrm{~F})$ and let $\mathcal{N}^{a} \subset \mathcal{N}$ be the fixed points sets. It is known that $\widetilde{S L}(\mathrm{~F})^{a}$ is a connected linear algebraic group and that $\mathcal{N}^{a}$ is an affine $\widetilde{S L}(\mathrm{~F})^{a}$-variety over $\mathbb{C}$. See $[\mathrm{V}]$.

Let $\mathcal{G}$ be the set of Lie subalgebras of $\mathfrak{s l}_{\mathrm{F}}$ which are $S L(\mathrm{~F})$-conjugate to $\mathfrak{s l}_{\mathrm{R}}$. The automorphism $a$ yields an automorphism of $\mathcal{G}$. Let $\mathcal{G}^{a} \subset \mathcal{G}$ be the fixed points set. We write

$$
\dot{\mathcal{N}}^{a}=\left\{(x, \mathfrak{p}) \in \mathcal{N}^{a} \times \mathcal{G}^{a} ; x \in \mathfrak{p}_{+}\right\},
$$


where $\mathfrak{p}_{+}$is the pro-nilpotent radical of $\mathfrak{p}$. We have $\mathcal{G}^{a}=\bigcup_{i \in \Xi} \mathcal{G}_{i}^{a}$ (a disjoint union), where each $\mathcal{G}_{i}^{a}$ is a smooth and connected $\widetilde{S L}(\mathrm{~F})^{a}$-variety. The set $\Xi$ is infinite. For each $i \in \Xi$ we put

$$
\dot{\mathcal{N}}_{i}^{a}=\dot{\mathcal{N}}^{a} \cap\left(\mathcal{N}^{a} \times \mathcal{G}_{i}^{a}\right)
$$

It is a smooth and connected $\widetilde{S L}(\mathrm{~F})^{a}$-variety. For each $i, j \in \Xi$ we also put

$$
\ddot{\mathcal{N}}_{i j}^{a}=\left\{\left(x, \mathfrak{p}, \mathfrak{p}^{\prime}\right) \in \mathcal{N}^{a} \times \mathcal{G}_{i}^{a} \times \mathcal{G}_{j}^{a} ; x \in \mathfrak{p}_{+} \cap \mathfrak{p}_{+}^{\prime}\right\}
$$

Let $K(X)$ be the complexified Grothendieck group of coherent sheaves over a scheme $X$. The $\mathbb{C}$-vector space

$$
\mathbb{S} \mathbb{K}=\prod_{j} \bigoplus_{i} K\left(\ddot{\mathcal{N}}_{i j}^{a}\right)
$$

is equipped with an associative multiplication called the convolution product. It is given the finite topology, i.e., the linear topology such that a basis of neighborhoods of 0 is formed by the subsets $\prod_{j \geqslant j_{0}} \bigoplus_{i} K\left(\ddot{\mathcal{N}}_{i j}^{a}\right)$. Note that the multiplication is continuous for this topology. We say that an $\mathbb{S} \mathbb{K}$-module $V$ is smooth if the annihilator of any element of $V$ in $\mathbb{S K}$ is open. The proposition is a direct consequence of the following lemma.

3.6.5. Lemma. Let $(\tau, \zeta) \in \Gamma_{c}(\mathbb{C})$ and $\mathrm{A}=\mathbb{C}$. Let $s \in H$ and let $\omega=\omega_{s, \tau} \subset H$ be the $\widetilde{\Sigma}_{n}$-orbit of $s$.

(a) There is a ring homomorphism $\mathbb{S H} \rightarrow \mathbb{S K}$ inducing an inclusion $\{$ smooth simple $\mathbb{S} \mathbb{K}$-modules $\} \subset\{$ simple $\mathbb{S H I}$-modules $\}$.

(b) Smooth simple $\mathbb{S} \mathbb{K}$-modules are labelled by $\widetilde{S L}(\mathrm{~F})^{a}$-orbits in $\mathcal{N}^{a}$.

(c) The integer $d_{\omega}(\mathbb{H})$ is the number of $\widetilde{S L}(\mathrm{~F})^{a}$-orbits in $\mathcal{N}^{a}$.

Proof. Part $(a)$ is proved in section A.16. Part $(c)$ is proved in [V, sec. 7.6]. Let us concentrate on part $(b)$.

Let $L_{i}$ be the direct image of the constant sheaf by the first projection

$$
p_{i}: \dot{\mathcal{N}}_{i}^{a} \rightarrow \mathcal{N}^{a} \text {. }
$$

Since $p_{i}$ is a proper map, the complex $L_{i}$ is a direct sum of shifted irreducible $\widetilde{S L}(\mathrm{~F})^{a}$-equivariant perverse sheaves. The set of smooth simple $\mathbb{S K}$-modules is in bijection with the set of indecomposable direct summands of $\bigoplus_{i} L_{i}$ (counted without multiplicities). See $\left[\mathrm{V}\right.$, sec. 6.1]. Let $\mathcal{O} \subset \mathcal{N}^{a}$ be a $\widetilde{S L}(\mathrm{~F})^{a}$-orbit. The intermediate extension of a nontrivial local system on $\mathcal{O}$ cannot occur in $\bigoplus_{i} L_{i}$. See [V, sec. 8.1]. It is enough to check that the intermediate extension of the trivial local system on $\mathcal{O}$ does occur.

To do so it is enough to prove that for some $i$ we have

$$
p_{i}\left(\dot{\mathcal{N}}_{i}^{a}\right)=\overline{\mathcal{O}}
$$

(the Zariski closure of $\mathcal{O}$ ). Indeed, in this case, for each $e \in \mathcal{O}$ the group of connected components of the centralizer of $e$ in $\widetilde{S L}(\mathrm{~F})^{a}$ acts on $H_{*}\left(p_{i}^{-1}(e), \mathbb{C}\right)$ and any irreducible submodule yields a $\widetilde{S L}(\mathrm{~F})^{a}$-equivariant irreducible local system on $\mathcal{O}$, whose intermediate extension to $\overline{\mathcal{O}}$ is a direct summand of $L_{i}$. Since $H_{*}\left(p_{i}^{-1}(e), \mathbb{C}\right) \neq 0$ and only the trivial local system can occur, we are done. 
Our proof of (3.6.6) is similar to $\mathrm{V}$, sec. 6.3]. We will be brief. For each $\mathfrak{p} \in \mathcal{G}^{a}$ let $\mathfrak{p}_{+}^{a}$ be the fixed points subset of the automorphism $a$ acting on $\mathfrak{p}_{+}$. Fix a group homomorphism

$$
v: \mathbb{C}^{\times} \rightarrow \mathbb{Q}, \quad v(\tau)<0<v(\zeta) .
$$

See [V, sec. 6.3, Claim 1]. Recall that $e \in \mathcal{O}$. Up to conjugating $e$ by an element in $\widetilde{S L}(\mathrm{~F})^{a}$ there is a $\mathfrak{s l}_{2}$-triple $\{e, f, h\} \subset \widetilde{\mathfrak{s l}}_{\mathrm{F}}$ which yields a group homomorphism

$$
\phi: S L_{2}(\mathbb{C}) \rightarrow \widetilde{S L}(\mathrm{~F})
$$

such that we have

$$
\phi(z):=\phi\left(\begin{array}{cc}
z & 0 \\
0 & 1 / z
\end{array}\right) \in \widetilde{H}(\mathbb{C}), \quad \forall z .
$$

See [V] sec. 6.3, Claim 2]. Put $s_{\phi}=s \phi\left(\zeta^{-1 / 2}\right)$. For each $u \in \mathbb{C}^{\times}, j \in \mathbb{Z}$ we set

$$
\mathfrak{s l}(u, j)=\left\{x(\varepsilon) \in \mathfrak{s l}_{\mathrm{F}} ;\left(\operatorname{ad} s_{\phi}\right)(x(\tau \varepsilon))=u x(\varepsilon),(\operatorname{ad} \phi(z))(x(\varepsilon))=z^{j} x(\varepsilon)\right\} .
$$

So we have $a(x)=\zeta^{j / 2-1} u x$ for each $x \in \mathfrak{s l}(u, j)$. Hence the parahoric Lie subalgebra

$$
\mathfrak{b}^{\prime}=\bigoplus_{v(u) \leqslant 0} \bigoplus_{j \in \mathbb{Z}} \mathfrak{s l}(u, j) \subset \mathfrak{s l}_{F}
$$

is $a$-fixed. So it is contained into a maximal parahoric Lie subalgebra $\mathfrak{p}^{\prime} \in \mathcal{G}^{a}$. Now we set

$$
\mathfrak{b}=(\operatorname{ad} \phi(\varepsilon))^{-1}\left(\mathfrak{b}^{\prime}\right), \quad \mathfrak{p}=(\operatorname{ad} \phi(\varepsilon))^{-1}\left(\mathfrak{p}^{\prime}\right) .
$$

There are inclusions

$$
\varepsilon \mathfrak{b} \subset \varepsilon \mathfrak{p} \subset \mathfrak{p}_{+} .
$$

Since $e \in \mathfrak{s l}(1,2)$ we have

$$
(\operatorname{ad} \phi(\varepsilon))(e) \in \varepsilon \mathfrak{s l}(\tau, 2) .
$$

Hence, since $v(\tau)<0$ we have

$$
e \in \varepsilon \mathfrak{b}, \quad e \in \mathfrak{p}_{+} .
$$

Next, since $\zeta^{m}=\tau^{k}$ we have

$$
\left(\mathfrak{b}^{\prime}\right)^{a}=\bigoplus_{r \geqslant 2} \mathfrak{s l}\left(\zeta^{1-r / 2}, r\right) .
$$

Since $(\operatorname{ad} \phi(\varepsilon))(\mathfrak{s l}(u, j))=\mathfrak{s l}\left(\tau^{j} u, j\right)$ we have also

$$
\mathfrak{b}^{a}=\bigoplus_{r(k-2 m) \geqslant 2 k} \mathfrak{s l}\left(\zeta^{1-r / 2}, r\right) .
$$

For each integer $r$ such that $r(k-2 m) \geqslant 2 k$ we have $r \geqslant 2$, because $0<-2 m<k$. Thus we have

$$
\mathfrak{b}^{a} \subset\left(\mathfrak{b}^{\prime}\right)^{a} .
$$

The proof of $\mathrm{V}$, sec. 6.3, Claim 3] implies that

$$
\left(\mathfrak{b}^{\prime}\right)^{a} \subset \overline{\mathcal{O}}
$$

(the Lie algebra $\mathfrak{b}^{\prime}$ coincides with the Lie algebra $\mathfrak{q}$ in [op. cit.]). Since $\mathfrak{b} \subset \mathfrak{p}$ we have $\mathfrak{p}_{+} \subset \mathfrak{b}_{+}$. Therefore we have

$$
\mathfrak{p}_{+}^{a} \subset \overline{\mathcal{O}} .
$$

By (3.6.7) and (3.6.8) there is a maximal parabolic Lie algebra $\mathfrak{p} \in \mathcal{G}$ such that $(e, \mathfrak{p}) \in \dot{\mathcal{N}}^{a}, \quad \widetilde{S L}(\mathrm{~F})^{a}\left(\mathfrak{p}_{+}^{a}\right) \subset \overline{\mathcal{O}}$. 
Fix $i \in \Xi$ such that $(e, \mathfrak{p}) \in \dot{\mathcal{N}}_{i}^{a}$. Then we have $e \in p_{i}\left(\dot{\mathcal{N}}_{i}^{a}\right)$. Thus we have also

$$
\mathcal{O} \subset p_{i}\left(\dot{\mathcal{N}}_{i}^{a}\right)
$$

Since $p_{i}$ is a proper map this yields

$$
\overline{\mathcal{O}} \subset p_{i}\left(\dot{\mathcal{N}}_{i}^{a}\right)
$$

Note that the inclusion $\widetilde{S L}(\mathrm{~F})^{a}\left(\mathfrak{p}_{+}^{a}\right) \subset \overline{\mathcal{O}}$ implies that $p_{i}\left(\dot{\mathcal{N}}_{i}^{a}\right) \subset \overline{\mathcal{O}}$. Thus we have proved (3.6.6).

We can now prove the main result of this section.

3.6.9. Theorem. Let $\mathrm{A}=\mathbb{C}$. Assume that $a_{o} \neq 0$ and $c<-2$. The rings $\mathbb{H}$, $\mathbb{S H}$ are Morita equivalent for each $(\tau, \zeta) \in \Gamma_{c}(\mathbb{C})$ except a finite number.

Proof. Recall that we omit the subscript $\mathbb{C}$ to simplify the notation. Set $M=$ $\mathbb{H} / \mathbb{H} o \mathbb{H}$. By $\underline{\mathrm{MR}}$, prop. 3.5.6] the $\mathbb{C}$-algebras $\mathbb{H}, \mathbb{S H}$ are Morita equivalent if $M=0$.

Assume initially that $\tau, \zeta$ are not roots of unity. Let $I \subset \mathbb{X}$ be an ideal such that $\mathbb{X} / I$ is an $\mathbb{X}$-module of finite length. By (3.1.1) the left $\mathbb{H}$-module $\mathbb{H} / \mathbb{H} I$ is filtered by the finite dimensional $\mathbb{X}$-modules $\sum_{\tilde{w}^{\prime} \leqslant \tilde{w}} t_{\tilde{w}^{\prime}} \mathbb{X} / I$ with $\tilde{w} \in \widetilde{\Sigma}_{n}$. Thus we have $\mathbb{H} / \mathbb{H} I \in \operatorname{Mod}(\mathbb{H})^{\prime}$. Hence we have also

$$
M / M I \in \operatorname{Mod}(\mathbb{H})^{\prime}
$$

Next, we have $F^{*}(M / M I)=0$ because $F^{*}$ is an exact functor and

$$
F^{*}(\mathbb{H})=o \mathbb{H}=o \mathbb{H} o \mathbb{H}=F^{*}(\mathbb{H} o \mathbb{H}) .
$$

Thus 3.6.2 implies that

$$
M / M I=0 .
$$

Now, observe that $M$ is an $\mathbb{X} \otimes \mathbb{Y}$-module of finite type, where $\mathbb{X}$ acts by right multiplication and $\mathbb{Y}$ by left multiplication. Assume that $M \neq 0$. Then there is a surjective $\mathbb{X} \otimes \mathbb{Y}$-module homomorphism $f: M \rightarrow \mathbb{C}$ by Zorn's lemma. Further, there is an ideal $I \subset \mathbb{X}$ as above such that

$$
M I \subset \operatorname{Ker}(f) .
$$

This is absurd because $M / M I=0$. Therefore we have

$$
M=0
$$

Now, set $\mathrm{A}=\mathbb{C}\left[\Gamma_{c}\right]$. Set also $M_{\mathrm{A}}=\mathbb{H}_{\mathrm{A}} / \mathbb{H}_{\mathrm{A}} o \mathbb{H}_{\mathrm{A}}$ as above. Since $M_{\mathrm{A}}$ is a $\mathbb{X}_{\mathrm{A}} \otimes \mathbb{Y}_{\mathrm{A}}$-module of finite type, by generic flatness there is an element $0 \neq f \in \mathrm{A}$ such that the localization $\left(M_{\mathrm{A}}\right)_{f}$ of $M_{\mathrm{A}}$ at $f$ is a free $\mathrm{A}_{f}$-module. See [E, thm. 14.4] for instance. Fix a $\mathbb{C}$-point $(\tau, \zeta) \in \Gamma_{c}(\mathbb{C})$. Note that $M_{\mathrm{A}} \otimes \mathbb{C}=M$ because taking tensor products with $\mathbb{C}$ is a right exact functor. Thus the previous discussion implies that $M_{\mathrm{A}} \otimes \mathbb{C}=0$ if $f(\tau, \zeta) \neq 0$ and $\tau, \zeta$ are not roots of unity. Thus we also have $M=0$ for all $(\tau, \zeta)$ such that $f(\tau, \zeta) \neq 0$. 


\section{Azumaya algebras over the Deformed Hilbert scheme}

4.1. Study of the deformed Hilbert schemes. In this section we study in detail the deformed Hilbert scheme $T$ introduced in section 2.6. Except when specified otherwise, we will assume that $\mathrm{A}=\mathbb{C}$. To unburden notation we will use the same symbol for a $\mathbb{C}$-scheme and its set of $\mathbb{C}$-points.

Set $D_{+}=G_{+} \times G_{+}$and $T_{\pi,+}=m_{+}^{-1}(0)$, the scheme-theoretic fiber of

$$
m_{+}: D_{+} \times T^{*} \mathbb{A}^{n} \rightarrow G_{+}, \quad\left(g, g^{\prime}, v, \varphi\right) \mapsto g g^{\prime}-\zeta^{2 l} g^{\prime} g+v \otimes \varphi .
$$

The schemes $T_{\pi}, T_{\pi,+}$ are closely related. There is an isomorphism

$$
T_{\pi} \rightarrow T_{\pi,+} \cap\left(D \times T^{*} \mathbb{A}^{n}\right), \quad(g, h, v, \varphi) \mapsto\left(g, h^{-1}, g h^{-1} v, \varphi\right) .
$$

Finally, we set $T_{\pi, \Sigma}=S_{\pi, \Sigma} \cap T_{\pi, \diamond}$.

4.1.1. Proposition. (a) The canonical map $\mathfrak{p}: T_{\pi, \diamond} \rightarrow T$ is a G-torsor.

(b) The scheme $T_{\pi,+}$ is a reduced complete intersection of pure dimension $2 n+$ $n^{2}$. If $\zeta^{2 l} \neq 1$, it is irreducible.

(c) The scheme $T$ is smooth, connected, of dimension $2 n$. The map $\mathfrak{q}: T \rightarrow N$ is a symplectic resolution of singularities of $N$. If $\zeta^{2 l n} \neq 1$, the map $\mathfrak{q}$ is an isomorphism. The scheme $N$ is integral, normal, of dimension $2 n$.

(d) The set $T_{\pi, \Sigma}$ is nonempty.

Before the proof we fix some notation. Let $G_{\star} \subset G$ be the set of invertible matrices which are conjugated to an element of

$$
H_{\star}=\left\{h \in H ; h^{\alpha} \neq 1, \zeta^{2 l}, \forall \alpha \in \Pi\right\} .
$$

Let $T_{\pi, \star} \subset T_{\pi}$ be the set of tuples $x=\left(g, g^{\prime}, v, \varphi\right)$ with $g \in G_{\star}$.

Let $T_{\pi}^{\prime}$ denote the variety $T_{\pi}$ with the parameter $\zeta$ replaced by $\zeta^{-1}$. We define $N^{\prime}$ in the same way. Consider the isomorphism

$$
\mathfrak{F}_{T}: T_{\pi}^{\prime} \rightarrow T_{\pi}, \quad x \mapsto\left(g^{\prime}, g,-\zeta^{-2 l} v, \varphi\right) .
$$

Set $T_{\pi, \odot}=T_{\pi, \star} \cup \mathfrak{F}_{T}\left(T_{\pi \star}^{\prime}\right)$. We define $N_{\odot}, T_{\odot}, N_{\star}$ and $T_{\star}$ in the obvious way.

Assume that $\zeta^{2 l} \neq 1$. Given $h \in H_{\star}, h^{\prime} \in H$ we set $x_{h, h^{\prime}}=\left(h, g^{\prime}, h^{\prime}, h\right)$ where the $(i, j)$-th entry of the matrix $g^{\prime}$ is $h_{i}^{\prime} h_{j} /\left(\zeta^{2 l} h_{j}-h_{i}\right)$. Note that $x_{h, h^{\prime}} \in T_{\pi, \star}$. The following is proved in section A.17.

4.1.2. Lemma. (a) Assume that $\zeta^{2 l} \neq 1$. We have $T_{\pi, \star} \subset T_{\pi, \diamond} \subset T_{\pi, \diamond}$. The assignment $\left(h, h^{\prime}\right) \mapsto x_{h, h^{\prime}}$ yields an isomorphism

$$
\left(H_{\star} \times H\right) / \Sigma_{n} \rightarrow N_{\star} .
$$

(b) The variety $T_{\pi, \diamond}$ is smooth and $G$-acts freely on it. The map $\mathfrak{q}$ gives isomorphisms of smooth varieties $T_{\odot} \rightarrow N_{\odot}, T_{\star} \rightarrow N_{\star}$. The Poisson bracket on $N_{\star}$ is the same as in $[\mathrm{FR}, \mathrm{O}]$.

Proof of 4.1.1. If $\zeta^{2 l}=1$, claims $(a)-(c)$ are known; see [GG, thm. 1.1.2] for part (b) and [N] for the other ones. If $\zeta^{2 l n} \neq 1$, then the following is proved in $[\mathrm{O}]$ :

- $T_{\pi}$ is a smooth $G$-torsor,

- $T_{\pi} / G$ is a smooth symplectic irreducible variety of dimension $2 n$.

Thus $T_{\pi, \diamond}=T_{\pi}$ and $T=T_{\pi} / G$. So claims $(a)-(c)$ are known again. Therefore, for all parts except $(d)$ we will assume that $\zeta^{2 l n}=1$ and $\zeta^{2 l} \neq 1$.

(a) See 4.1.2(a).

(b) See section A.17. 
(c) The smoothness of $T$ follows from 4.1.2(a). By 4.1.2(b) the Poisson bracket on $T$ is nondegenerate over the open subset $T_{\odot}$. Thus $T$ is a symplectic variety, because $\operatorname{codim}\left(T \backslash T_{\odot}\right) \geqslant 2$.

The variety $T_{\pi}$ is normal, because it is a complete intersection, the open subset $T_{\pi, \odot}$ is smooth by 4.1.2(a), and $\operatorname{codim}\left(T_{\pi} \backslash T_{\pi, \odot}\right) \geqslant 2$. Thus $N$ is irreducible and normal.

(d) First, recall that $T_{\pi, \Sigma}$ is the set of tuples $\left(g, g^{\prime}, v, \varphi\right) \in S_{\pi, \diamond}$ such that

$$
e+v \otimes \varphi=\zeta^{2 l}\left[g, g^{\prime}\right]^{-1}, \quad g^{\prime}, g g^{\prime} g^{-1}, \zeta^{-2 l}\left[g, g^{\prime}\right] \in G_{\Sigma}, \quad \varphi_{i} \neq 0,
$$

where $G_{\Sigma}=U_{+} H U_{-}$. Define the following open sets:

$$
\begin{aligned}
& U=\left\{x \in T_{\pi} ; g^{\prime}, g g^{\prime} g^{-1} \in G_{\Sigma}, \varphi_{i} \neq 0\right\}, \\
& V=\left\{x \in T_{\pi} ; \zeta^{-2 l}\left[g, g^{\prime}\right] \in G_{\Sigma}\right\} .
\end{aligned}
$$

First, assume that $\zeta^{2 l} \neq 1$. Fix a tuple $x_{h, h^{\prime}}=\left(h, g^{\prime}, h^{\prime}, h\right)$ as above. The principal minors of the matrix $g^{\prime}$ are nonzero, see [0, sec. 2.2]. Hence $g^{\prime} \in G_{\Sigma}$. Thus $x_{h, h^{\prime}} \in U$. Any element of $G$ is conjugate to an element of $G_{\Sigma}$. Thus the $G$-orbit of $x_{h, h^{\prime}}$ contains a representative in $V$. Thus $U, V \neq \emptyset$. We have $T_{\pi, \Sigma}=U \cap V \cap T_{\pi, \diamond}$. We are done, because $T_{\pi, \diamond}$ is open and nonempty and $T_{\pi}$ is irreducible.

Next, assume that $\zeta^{2 l}=1$. Set $x=\left(h, h^{\prime}, 0, \varphi\right)$ with $h \in H_{\star}$ and $h^{\prime}, \varphi \in H$. We have $x \in T_{\pi, \Sigma}$.

The following is proved in section A.17.

4.1.3. Proposition. The $\mathcal{L}$-module $\mathcal{O}(N)$ is of finite type.

4.1.4. Remark. We have seen that the quasi-projective variety $T$ is affine if $\zeta^{2 l n} \neq$ 1 , but not if $\zeta^{2 l}=1$. One can prove that if $\zeta^{2 l n}=1$, then $T$ is not affine.

4.2. Azumaya algebras over the deformed Hilbert scheme. In this section we set $\mathrm{A}=\mathbb{C}$ and $l=p^{e}$.

4.2.1. Theorem. Assume that $(\tau, \zeta) \in \Gamma_{c}(\mathbb{C})$ and that $p$ is large enough.

(a) The sheaf $\mathfrak{T}$ over $T$ is an Azumaya algebra of PI-degree $l^{n}$.

(b) Assume that $c<-2,2 n<k$ and $\zeta^{2 l}=1$. We have $\mathfrak{T}(T) \simeq \mathbf{S H}$ as $\mathcal{L}$-algebras and $H^{i}(T, \mathfrak{T})=0$ for all $i>0$.

Proof. (a) Note that $T$ is a symplectic variety by $4.1 .1(c)$ and that $\mathfrak{T}\left(T_{t}\right)$ is a Poisson $\mathcal{O}\left(T_{t}\right)$-order for each $t$ by 2.7.6. Thus $\mathfrak{T}$ is a locally free $\mathcal{O}_{T}$-module with isomorphic fibers (as algebras) by A.10.3(a). We must prove that $\mathfrak{T}$ is an Azumaya algebra over $T$. Recall that a coherent sheaf of $\mathcal{O}_{T}$-algebras over $T$ is an Azumaya algebra iff it is a locally-free $\mathcal{O}_{T}$-module with fibers isomorphic to matrix algebras; see M2, IV.2.1]. We set

$$
T_{\Sigma}=\mathfrak{p}\left(T_{\pi, \Sigma}\right),
$$

a nonempty open subset of $T$ by 4.1.1(d). It is enough to prove that $\mathfrak{T}$ is an Azumaya algebra over $T_{\Sigma}$. The fibers of $\mathfrak{S}_{\pi}$ over $S_{\pi, \Sigma}$ are matrix algebras by 2.5.6(d). By 1.5.2(b) the fibers of $\mathfrak{T}_{\pi}$ over $T_{\pi, \Sigma}$ are also matrix algebras. So $\mathfrak{T}$ is an Azumaya algebra over $T_{\Sigma}$ by faithfully flat descent and 2.7.6, 4.1.1(a). 
Now, we compute the PI-degree of $\mathfrak{T}$. Let $(\tau, \zeta) \in \Gamma_{c}(\mathbb{C})$. Recall that $\mathfrak{T}_{c}$ is the sheaf of $\mathcal{O}_{T_{c}}$-algebras associated with the $\mathbb{Z}_{+}$-graded $\mathcal{O}\left(T_{\pi, c}\right)^{+}$-module $\mathbf{T}_{c}^{+}$. By 2.3.6(c) and 2.6.11(a) we have $\mathbf{T}_{c}^{+} \otimes \mathrm{k}_{c}=\mathcal{O}\left(T_{\pi, \mathbf{k}_{c}}\right)^{+}$. Recall the subring

$$
\mathcal{O}\left(T_{\pi, \mathrm{k}_{c}}\right)^{l}=\mathcal{O}\left(T_{\pi, \mathrm{k}_{c}}^{(e)}\right) \subset \mathcal{O}\left(T_{\pi, \mathrm{k}_{c}}\right)
$$

see (1.2.1), (1.2.2). By 2.6.2(d) we have also

$$
T_{c} \otimes \mathrm{k}_{c}=\left(T_{\mathrm{k}_{c}}\right)^{(e)}=\operatorname{Proj}\left(\mathcal{O}\left(T_{\pi, \mathrm{k}_{c}}\right)^{l,+}\right) \text {. }
$$

Thus $\mathfrak{T}_{c} \otimes \mathrm{k}_{c}$ is the sheaf of $\mathcal{O}_{T_{\mathbf{k}_{c}}^{(e)}}$-algebras associated with the $\mathbb{Z}_{+}$-graded $\mathcal{O}\left(T_{\pi, \mathrm{k}_{c}}\right)^{l,+}$ algebra $\mathcal{O}\left(T_{\pi, \mathrm{k}_{c}}\right)^{+}$. In other words, we have an isomorphism of sheaves of algebras

$$
\mathfrak{T}_{c} \otimes \mathrm{k}_{c}=\left(F r^{e}\right)_{*} \mathcal{O}_{T_{\mathrm{k}_{c}}} \text {. }
$$

Next, recall that $\mathrm{A}_{c} \subset \mathbb{C}$. Thus, by base change, to compute the PI-degree of $\mathfrak{T}$ it is enough to prove that the $\mathcal{O}_{T_{c}}$-module $\mathfrak{T}_{c}$ is locally-free of rank $l^{2 n}$.

Note that $\mathfrak{T}_{c}, \mathcal{O}_{T_{c}}$ are flat $\mathrm{A}_{c}$-modules by 2.6.2(d), 2.7.3(a). Note also that $\mathfrak{T}_{c}$ is a finitely generated $\mathcal{O}_{T_{c}}$-module. Furthermore, $\left(\mathrm{Fr}^{e}\right)_{*} \mathcal{O}_{T_{\mathrm{k}_{c}}}$ is a locally free $\mathcal{O}\left(T_{\mathrm{k}_{c}}^{(e)}\right)$-module of rank $l^{2 n}$, because $T_{\mathrm{k}_{c}}$ is a smooth variety. Thus $\mathfrak{T}_{c} \otimes \mathrm{k}_{c}$ is a locally-free $\mathcal{O}_{T_{c}} \otimes \mathrm{k}_{c}$-module of rank $l^{2 n}$. Finally, $\mathfrak{T}_{c} \otimes \mathrm{k}_{c}$ is a flat $\mathcal{O}_{T_{c}} \otimes \mathrm{k}_{c}$-module, because $\mathfrak{T}$ is a flat $\mathcal{O}_{T}$-module. Thus $\mathfrak{T}_{c}$ is a locally-free $\mathcal{O}_{T_{c}}$-module of rank $l^{2 n}$ by the following lemma, proved in section A.18.

4.2.2. Lemma. Let $\mathrm{A}$ be a DVR with residue field $\mathrm{k}$ and fraction field $\mathrm{k}$. Let $\mathrm{B}$ be an A-algebra which is a CNID and let $M$ be a $\mathrm{B}$-module. Assume that $\mathrm{B}, M$ are flat $\mathrm{A}$-modules and that $M$ is a $\mathrm{B}$-module of finite type. If $M \otimes \mathrm{k}$ is a flat $\mathrm{B} \otimes \mathrm{k}-m o d u l e$ and $M \otimes \mathrm{k}$ is a flat $\mathrm{B} \otimes \mathrm{k}$-module, then $M$ is a flat $\mathrm{B}-m o d u l e$.

(b) Now we concentrate on the second claim. The proof consists of 3 parts.

Step 1 . We will omit the subscript $\mathbb{C}$ for $\mathbb{C}$-schemes. First, we prove that

$$
H^{>0}(T, \mathfrak{T})=0 .
$$

By 2.3.6(c), 2.6.2(d), 2.6.11(a), 2.7.1(c) there is a graded $\mathrm{k}_{c}$-algebra homomorphism

$$
\mathcal{O}\left(T_{\pi, \mathrm{k}_{c}}^{(e)}\right)^{+}=\mathcal{O}\left(T_{\pi, \mathrm{k}_{c}}\right)^{l,+} \rightarrow \mathbf{T}_{c}^{+} \otimes \mathrm{k}_{c}=\mathcal{O}\left(T_{\pi, \mathrm{k}_{c}}\right)^{+}
$$

Furthermore, $\mathcal{O}\left(T_{\pi, \mathrm{k}_{c}}\right)^{+}$is generated by $\mathcal{O}\left(T_{\pi, \mathrm{k}_{c}}\right)^{1}$ as an $\mathcal{O}\left(T_{\pi, \mathrm{k}_{c}}\right)^{0}$-algebra by A.17.4. Finally, $\mathfrak{T}_{c} \otimes \mathrm{k}_{c}$ is the sheaf over $T_{c} \otimes \mathrm{k}_{c}$ associated with the graded $\mathcal{O}\left(T_{\pi, \mathrm{k}_{c}}^{(e)}\right)^{+}$-module $\mathbf{T}_{c}^{+} \otimes \mathrm{k}_{c}$. Therefore 4.2.4(a) below, proved in section A.18, yields a $\mathbb{Z}_{+}$-graded $\mathrm{k}_{c}$-algebra isomorphism

$$
\begin{aligned}
H^{\bullet}\left(T_{c} \otimes \mathrm{k}_{c}, \mathfrak{T}_{c} \otimes \mathrm{k}_{c}\right) & =H^{\bullet}\left(\operatorname{Proj}\left(\mathcal{O}\left(T_{\pi, \mathrm{k}_{c}}^{(e)}\right)^{+}\right), \mathfrak{T}_{c} \otimes \mathrm{k}_{c}\right) \\
& =H^{\bullet}\left(\operatorname{Proj}\left(\mathbf{T}_{c}^{+} \otimes \mathrm{k}_{c}\right), \mathcal{O}\right) \\
& =H^{\bullet}\left(T_{\mathrm{k}_{c}}, \mathcal{O}\right) .
\end{aligned}
$$

Now, recall that $T_{\mathrm{k}_{c}}$ is the punctual Hilbert scheme of the $\mathrm{k}_{c}$-scheme $\mathbb{G}_{m, \mathrm{k}_{c}} \times \mathbb{G}_{m, \mathrm{k}_{c}}$. So BK2] and the equality above yield

$$
H^{>0}\left(T_{c} \otimes \mathrm{k}_{c}, \mathfrak{T}_{c} \otimes \mathrm{k}_{c}\right)=0 .
$$

Next, assume temporarily that $\mathrm{A}=\mathbb{C}$. By 4.1.1(c) the $\mathbb{C}$-algebra $\mathcal{O}(N)$ is equipped with a Poisson bracket. Since $\zeta^{2 l}=1$, by 4.1.2(b) this Poisson bracket is the same as the Poisson bracket considered in [BG2, sect. 7.4]. By [loc. cit.] the following hold: there is a finite number of symplectic leaves $N^{(1)}, N^{(2)}, \ldots, N^{(r)}$, 
for each $i$ the leaf $N^{(i)}$ is a locally closed subset of $N$ which is defined over $\mathrm{A}_{c}$ and we have $\bar{N}^{(i)}\left(\mathrm{k}_{c}\right) \neq \emptyset$. Therefore 2.6.2(d), 2.7.3(a), 2.7.6 and 4.2.4(b) imply (4.2.3).

4.2.4. Lemma. (a) Let $\mathrm{k}$ be a field and let $f: \mathrm{A} \rightarrow \mathrm{B}$ be a morphism of $\mathbb{Z}_{+-}$-graded commutative $\mathrm{k}$-algebras. Assume that $\mathrm{A}^{m}=0$ if $m / l \notin \mathbb{Z}$, that $\mathrm{A}$ is generated by $\mathrm{A}^{l}$ as an $\mathrm{A}^{0}$-algebra and that $\mathrm{B}$ is generated by $\mathrm{B}^{1}$ as a $\mathrm{B}^{0}$-algebra. Set $X=\operatorname{Proj}(\mathrm{A})$, $Y=\operatorname{Proj}(\mathrm{B})$ and let $\mathcal{E}$ be the $\mathcal{O}_{X}$-algebra associated with the graded A-algebra $\mathrm{B}$. Then there is a $\mathbb{Z}_{+}$-graded $\mathrm{k}$-algebra isomorphism $H^{\bullet}(X, \mathcal{E}) \simeq H^{\bullet}\left(Y, \mathcal{O}_{Y}\right)$.

(b) Let $\mathrm{A} \subset \mathbb{C}$ be a DVR with residue field $\mathrm{k}$. Let $f: X \rightarrow Y$ be a proper morphism of Poisson A-schemes of finite type. Let $Y=\bigcup_{i=1}^{r} Y_{i}$ be a stratification of $Y$ into locally closed subsets such that $\bar{Y}_{i}(\mathrm{k}) \neq \emptyset$ for each $i$. Let $\mathcal{E} \in \operatorname{Coh}\left(\mathcal{O}_{X}\right)$ be an $\mathcal{O}_{X}$-algebra. Assume that $Y$ is affine and irreducible and that $X, \mathcal{E}$ are flat over A. Assume also that $\left\{\mathrm{Y}_{i} \otimes \mathbb{C}\right\}$ is the set of symplectic leaves of $Y \otimes \mathbb{C}$ and that $\mathcal{E} \otimes \mathbb{C}$ is a Poisson order over $f$. Then we have

$$
H^{>0}(X \otimes \mathrm{k}, \mathcal{E} \otimes \mathrm{k})=0 \Rightarrow H^{>0}(X \otimes \mathbb{C}, \mathcal{E} \otimes \mathbb{C})=0 .
$$

Step 2. Now, we construct a surjective $\mathcal{L}$-algebra homomorphism

$$
\mathfrak{T}(T) \rightarrow \mathbf{S H}
$$

Recall that $\mathfrak{T}$ is the coherent sheaf over $T$ associated with the $\mathbb{Z}_{+}$-graded $\mathcal{O}\left(T_{\pi}\right)^{+}$module $\mathbf{T}^{+}$. So there is a $\mathcal{L}$-algebra homomorphism $\mathbf{T}^{0} \rightarrow \mathfrak{T}(T)$. Recall the $\mathcal{L}$ algebra homomorphism $\Phi: \mathfrak{T}\left(T_{\star}\right) \rightarrow \mathbf{D}_{0, \star}^{\Sigma_{n}}$ from 2.8.1(a). Apply it to the diagram

$$
\mathbf{T}^{0} \rightarrow \mathfrak{T}(T) \rightarrow \mathfrak{T}\left(T_{\star}\right) .
$$

We get the chain of $\mathcal{L}$-algebras

$$
\Phi\left(\mathbf{T}^{0}\right) \subset \Phi(\mathfrak{T}(T)) \subset \mathbf{D}_{0, \star}^{\Sigma_{n}}
$$

Now, the $\mathcal{L}$-algebra homomorphism $\Psi: \mathbf{S H} \rightarrow \mathbf{D}_{0, \star}^{\Sigma_{n}}$ is injective. Furthermore, 3.3.1 and 3.5.1 yield the following $\mathcal{L}$-algebra inclusions

$$
\Psi(\mathbf{S H}) \subset \Phi\left(\mathbf{T}^{0}\right) \subset \Psi\left(\mathbf{S H}_{\star}\right)=\mathbf{D}_{0, \star}^{\Sigma_{n}} .
$$

Therefore we have constructed $\mathcal{L}$-algebra embeddings

$$
\mathbf{S H} \subset \Phi(\mathfrak{T}(T)) \subset \mathbf{S H}_{\star} .
$$

Finally, $\mathfrak{T}(T)$ is a $\mathcal{L}$-module of finite type by 4.1 .3 , because $\mathfrak{T}$ is a coherent sheaf over $T$. Since $\mathbf{S H}$ is a maximal order by 3.4.2, we have $\mathbf{S H} \simeq \Phi(\mathfrak{T}(T))$ by A.10.1(a).

Step 3. Finally, we prove that (4.2.5) is injective. It is enough to prove that the map $\Phi: \mathfrak{T}\left(T_{\star}\right) \rightarrow \mathbf{D}_{0, \star}^{\Sigma_{n}}$ is injective. Recall that $\Psi: \mathbf{S H} \rightarrow \mathbf{D}_{0, \star}^{\Sigma_{n}}$ is an $\mathcal{L}$-algebra embedding. Under restriction and localization the map $\Phi$ yields a Poisson algebra homomorphism

$$
\phi: \mathcal{O}\left(T_{\star}\right) \rightarrow \mathcal{D}_{0, \star}^{\Sigma_{n}}
$$

see 2.8.1(b). The kernel of $\phi$ is a Poisson ideal. It is well known that if $X$ is a smooth, connected, affine and symplectic variety over $\mathbb{C}$, then the Poisson algebra $\mathcal{O}(X)$ has no proper Poisson ideals. Thus $\phi$ is injective by $4.1 .1(c)$.

Since $\mathfrak{T}$ is an Azumaya algebra over $T$, we have that $\mathfrak{T}\left(T_{\star}\right)$ is an Azumaya algebra over $\mathcal{O}\left(T_{\star}\right)$. Hence, extensions and contractions give a one-to-one correspondence between 2-sided ideals of $\mathfrak{T}\left(T_{\star}\right)$ and ideals of $\mathcal{O}\left(T_{\star}\right)$; see [MR, prop. 13.7.9]. Thus $\Phi$ is also injective. 
We will end this section with an application of 4.2.1 to the representation theory of the DAHA. Before that, we need the following.

4.2.6. Proposition. Under the assumptions of 4.2.1 the varieties $C M, N$ are isomorphic.

Proof. The map $\mathfrak{q}$ yields an isomorphism $\mathcal{O}(N) \simeq \mathcal{O}(T)$ because $N$ is a normal variety; see 4.1.1(c). By 4.2.1(a) we have $\mathcal{O}(T) \subset Z \mathfrak{T}(T) \subset \mathfrak{T}(T)$. By 4.2.1(b) we have $Z \mathfrak{T}(T) \simeq Z(\mathbf{S H})$. By the definition of $C M$ we have $Z(\mathbf{S H})=\mathcal{O}(C M)$. Thus we have

$$
\mathcal{O}(N) \subset \mathcal{O}(C M) \subset \mathfrak{T}(T) .
$$

Hence $\mathcal{O}(C M)$ is a $\mathcal{O}(N)$-submodule of $\mathfrak{T}(T)$. Furthermore, $\mathfrak{T}(T)$ is a $\mathcal{O}(N)$-module of finite type, because $\mathfrak{T}$ is a coherent sheaf over $T$ and $\mathfrak{q}$ is a proper map. So $\mathcal{O}(C M)$ is also a $\mathcal{O}(N)$-module of finite type.

Since $T_{\star} \subset T$ is an irreducible affine open subset, we have

$$
\mathcal{O}(N) \subset \mathcal{O}(C M) \subset Z \mathfrak{T}\left(T_{\star}\right)=\mathcal{O}\left(T_{\star}\right)=\mathcal{O}\left(N_{\star}\right) \subset \mathrm{k}(N) .
$$

Thus $C M \simeq N$, because $N$ is a normal variety.

Now, fix a $\mathbb{C}$-point $x \in N(\mathbb{C})$. Let $\operatorname{Coh}_{x}\left(\mathcal{O}_{T}\right)$ be the category consisting of coherent sheaves of $\mathcal{O}_{T}$-modules on the formal neighborhood of $\mathfrak{q}^{-1}(x)$ in $T$. We can view $x$ as a $\mathbb{C}$-point of $C M$ by 4.2.6. Let $\mathcal{M}_{\boldsymbol{o}} \boldsymbol{d}_{x}^{\mathrm{fg}}(\mathbf{H})$ be the category of finitely generated complete topological modules over the adic completion of $\mathbf{H}$ with respect to the maximal ideal of $Z(\mathbf{S H})$ associated with $x$. Using 3.1.2, 3.6.9, 4.2.1 and 4.2.6, the same techniques as in [BFG], BK1] give the following (see the introduction and section A.18 for details).

4.2.7. Theorem. Under the assumptions of 4.2.1 there is an equivalence of triangulated categories $D^{b}\left(\boldsymbol{C o h}_{x}\left(\mathcal{O}_{T}\right)\right) \simeq D^{b}\left(\boldsymbol{M o d}_{x}^{\mathrm{fg}}(\mathbf{H})\right)$.

\subsubsection{Remarks.}

(a) For each $n$, if we set $m=-n, k>2 n>2,(p, n)=1, \tau=u^{m}$ and $\zeta=u^{k}$, where $u$ is a primitive $m l$-th root of unity, then we obtain $\zeta^{2 l n}=1 \neq \zeta^{2 l}$ for each $l$.

(b) The restriction to $\zeta^{2 l}=1$ in 4.2 .1 can be removed by the same method.

(c) Note that [BFG] gives only a splitting of the Azumaya algebra $\mathfrak{T}$ over $\mathfrak{q}^{-1}(x)$ for Hilbert schemes, i.e., if we have $\zeta^{2 l}=1$. See section A.18. The splitting of $\mathfrak{T}$ for deformed Hilbert schemes deserves a special study.

(d) Two complex numbers $\zeta, \zeta^{\prime} \neq 0$ such that $\zeta^{l}=\left(\zeta^{\prime}\right)^{l}$ yield Morita equivalent Azumaya algebras over the same scheme $T$.

\section{A. Appendix}

\section{A.1. Proof of 1.3 .}

Proof of 1.3.3(c). Write ad ${ }^{[i]}$ for the adjoint action in $\mathbf{U}^{[i]}$. By 1.3.2(a) we have the following relation in $\mathbf{U}^{[2]}$ :

$$
(\text { id } \otimes \iota) \Delta\left(u \otimes u^{\prime}\right)=\sum_{x, y, s, t} u_{1} \otimes r_{s}^{+} u_{1}^{\prime} r_{t}^{+} \otimes r_{x}^{-} \iota\left(r_{t}^{-}\right) \iota\left(u_{2}\right) r_{s}^{-} r_{y}^{-} \otimes r_{x}^{+} \iota\left(u_{2}^{\prime}\right) \iota\left(r_{y}^{+}\right) .
$$

Using this formula, a direct computation yields

$$
\operatorname{ad}^{[2]}\left(u \otimes u^{\prime}\right)=\varpi_{2} \circ\left(\operatorname{ad}(u) \otimes \operatorname{ad}\left(u^{\prime}\right)\right) \circ \varpi_{2}^{-1},
$$


where $\varpi_{2}$ is the linear map

$$
\varpi_{2}: \mathbf{U}^{\otimes 2} \rightarrow \mathbf{U}^{[2]}, v \otimes v^{\prime} \mapsto v r_{s}^{-} \otimes\left(\operatorname{ad} r_{s}^{+}\right)\left(v^{\prime}\right) .
$$

In the same way, in $\mathbf{U}^{[3]}$ we have

$$
\operatorname{ad}^{[3]}\left(u \otimes u^{\prime}\right)=\bar{\varpi}_{3} \circ\left(\left(\operatorname{ad}^{[2]} u\right) \otimes\left(\operatorname{ad} u^{\prime}\right)\right) \circ \bar{\varpi}_{3}^{-1},
$$

where $\bar{\varpi}_{3}$ is the linear map $\mathbf{U}^{[2]} \otimes \mathbf{U} \rightarrow \mathbf{U}^{[3]}, v \otimes v^{\prime} \mapsto v \Delta\left(r_{s}^{-}\right) \otimes\left(\operatorname{ad} r_{s}^{+}\right)\left(v^{\prime}\right)$. Set

$$
\varpi_{3}=\bar{\varpi}_{3} \circ\left(\varpi_{2} \otimes \mathrm{id}\right): \mathbf{U}^{\otimes 3} \rightarrow \mathbf{U}^{[3]} .
$$

For future use, notice that

$$
\begin{aligned}
& \varpi_{3}(u \otimes v \otimes w)=u r_{s}^{-} \otimes\left(\operatorname{ad}^{[2]} \Delta r_{s}^{+}\right) \varpi_{2}(v \otimes w), \\
& \operatorname{ad}^{[2]}\left(u \otimes u^{\prime}\right)(v \otimes 1)=(\operatorname{ad} u)(v) \otimes \varepsilon\left(u^{\prime}\right), \\
& \operatorname{ad}^{[3]}\left(u \otimes u^{\prime}\right)(v \otimes 1)=\left(\operatorname{ad}^{[2]} u\right)(v) \otimes \varepsilon\left(u^{\prime}\right) .
\end{aligned}
$$

Now we concentrate on 1.3.3(c). We prove only the first part. We have

$$
\kappa(f)=R^{-} \iota\left(f_{1}\right) R^{+}\left(f_{2}\right) \Rightarrow \Delta \kappa(f)=R^{-} \iota\left(f_{1}\right) R^{+}\left(f_{3}\right) \otimes \kappa\left(f_{2}\right) .
$$

Thus

$$
\begin{aligned}
\varpi_{2}(\kappa \otimes \kappa) \Delta(f) & =\kappa\left(f_{1}\right) r_{s}^{-} \otimes \kappa\left(\left(\operatorname{ad} r_{s}^{+}\right)\left(f_{2}\right)\right) \\
& =\kappa\left(f_{1}\right) r_{s}^{-} r_{t}^{-} \otimes\left(f_{2}: \iota r_{s}^{+}\right)\left(f_{4}: r_{t}^{+}\right) \kappa\left(f_{3}\right) \\
& =\kappa\left(f_{1}\right) R^{+} \iota\left(f_{2}\right) R^{+}\left(f_{4}\right) \otimes \kappa\left(f_{3}\right) \\
& =\Delta \kappa(f) .
\end{aligned}
$$

\section{A.2. Proof of 1.4 .}

Proof of $1.4 .1(b),(c)$. It is routine to check that $\varpi_{2}: \mathbf{U}^{(2)} \rightarrow \mathbf{U}^{[2]}$ is an algebra isomorphism, because the multiplication of $u \otimes u^{\prime}$ and $v \otimes v^{\prime}$ in $\mathbf{U}^{(2)}$ is equal to $u\left(\operatorname{ad} r_{s}^{-}\right)(v) \otimes\left(\operatorname{ad} r_{s}^{+}\right)\left(u^{\prime}\right) v^{\prime}$. The rest follows from [DM, 4.17], [BS].

Proof of 1.4.2. Put $c=\sum_{s} a_{s} \otimes b_{s}, c^{-1}=\sum_{s} \bar{a}_{s} \otimes \bar{b}_{s}$ and $\Delta^{c}(h)=h_{1}^{c} \otimes h_{2}^{c}$. The assignment

$$
a \otimes h \mapsto \sum_{s}\left(a_{s} \triangleright a\right) \otimes\left(b_{s} h\right)
$$

yields an algebra isomorphism $\Xi: \mathbf{A}_{c} \sharp \mathbf{H}_{c} \rightarrow \mathbf{A} \sharp \mathbf{H}$, because

$$
\sum_{s}\left(a_{s} h_{1}^{c} \triangleright a\right) \otimes\left(b_{s} h_{2}^{c}\right)=\sum_{s}\left(h_{1} a_{s} \triangleright a\right) \otimes\left(h_{2} b_{s}\right) .
$$

The inverse is given by

$$
\Xi^{-1}\left(\ell(a) \partial_{\triangleright}(h)\right)=\sum_{s} \ell\left(\bar{a}_{s} \triangleright a\right) \partial_{\triangleright}\left(\bar{b}_{s} h\right)
$$

Identify $\mathbf{A}_{c}$, A with the quotients $\mathbf{A}_{c} \sharp \mathbf{H}_{c} / \mathbf{A}_{c} \sharp \mathbf{H}_{c}^{\varepsilon}$ and $\mathbf{A} \sharp \mathbf{H} / \mathbf{A} \sharp \mathbf{H}^{\varepsilon}$, respectively. By definition of a twist we have $\sum_{s} a_{s} \varepsilon\left(b_{s}\right)=1$. Thus the map $\Xi$ factors to the identity $\mathbf{A}_{c} \rightarrow \mathbf{A}$. 
Proof of 1.4.3(c). We have $\mathbf{U} \subset \mathbf{F}^{*}$. Thus an element $d=\sum_{i} f_{i} \otimes u_{i}$ may be identified with the linear operator

$$
d^{\prime}: \mathbf{F} \rightarrow \mathbf{F}, g \mapsto \sum_{i} f_{i} u_{i}(g) .
$$

Next, let $d^{\prime \prime}: \mathbf{F} \rightarrow \mathbf{F}$ be the action of $d$ in the basic representation. We must check that $d^{\prime}=0$ if $d^{\prime \prime}=0$. This follows from the formula

$$
d^{\prime \prime}\left(\iota g_{1}\right) g_{2}=\sum_{i}\left(\iota g_{1}: u_{i}\right) f_{i} \iota\left(g_{2}\right) g_{3}=d^{\prime}(\iota g), \quad \forall g \in \mathbf{F} .
$$

\section{A.3. Proof of 1.5 .}

Proof of 1.5.2. (a) The left ideal $\mathbf{I}=\mathbf{A} \partial_{\triangleright}\left(\mathbf{H}^{\prime}\right)^{\chi}$ of $\mathbf{A}$ is $\mathbf{H}^{\prime}$-stable. For each $a \in \mathbf{A}$ we have

$$
\mathbf{I} a \subset \mathbf{I} \Longleftrightarrow\left\{\left(h_{1} \triangleright a\right) \partial_{\triangleright}\left(h_{2}\right) ; h \in\left(\mathbf{H}^{\prime}\right)^{\chi}\right\} \subset \mathbf{I} .
$$

As $\mathbf{H}^{\prime}$ is $\chi$-stable the automorphism $h \mapsto h^{\chi}$ maps $\left(\mathbf{H}^{\prime}\right)^{\chi}$ onto $\left(\mathbf{H}^{\prime}\right)^{\varepsilon}$. Since $\partial_{\triangleright}(h)=$ $\chi(h)$ modulo I we have

$$
\mathbf{I} a \subset \mathbf{I} \Longleftrightarrow\left(\mathbf{H}^{\prime}\right)^{\varepsilon} \triangleright a \subset \mathbf{I} \Longleftrightarrow a+\mathbf{I} \in(\mathbf{A} / \mathbf{I})^{\mathbf{H}^{\prime}} .
$$

So the evaluation at the element $1+\mathbf{I}$ yields an isomorphism $\operatorname{End}_{\mathbf{A}}(\mathbf{A} / \mathbf{I}) \rightarrow$ $(\mathbf{A} / \mathbf{I})^{\mathbf{H}^{\prime}}$. In particular, the right-hand side is a ring such that $(a+\mathbf{I})\left(a^{\prime}+\mathbf{I}\right)=a a^{\prime}+\mathbf{I}$.

(b) Assume that $\mathbf{A}=\operatorname{End}(V)$, with $V$ a finite dimensional vector space. View $V$ is a $\mathbf{H}^{\prime}$-module via $\partial_{\triangleright}$. Set $W=\{v \in V ; \mathbf{I} \cdot v=0\}$. Since $\mathbf{I} \subset \operatorname{End}(V)$ is a left ideal, it is equal to $\{a \in \mathbf{A} ; a \cdot W=0\} \simeq \operatorname{Hom}(V / W, V)$. Hence

$$
\mathbf{A} / / \chi \mathbf{H}^{\prime}=(\mathbf{A} / \mathbf{I})^{\mathbf{H}^{\prime}}=\operatorname{Hom}(W, V)^{\mathbf{H}^{\prime}}=\operatorname{End}(W) .
$$

Part $(c)$ is obvious.

\section{A.4. Proof of 1.7 .}

Proof of 1.7.2. (a) We will prove that $\kappa \iota\left(c_{i j}\right) \in \mathbb{U}_{\pi}^{\prime}$ for all $j \neq 1$. The rest of the claim is left to the reader. First we fix $\lambda \in X_{+}$and $c_{\varphi, v} \in \operatorname{cf}(V(\lambda))$. Assume that $\varphi, v$ are homogeneous of weight $\lambda-\alpha, \lambda-\gamma$, respectively. By A.4.1 below we have

$$
\kappa\left(c_{\varphi, v}\right) \in \bigoplus_{0 \leqslant \beta \leqslant \alpha, \gamma}\left(\mathbb{U}_{+}\right)_{\alpha-\beta} k_{2 \lambda-2 \beta} \iota\left(\mathbb{U}_{-}\right)_{\beta-\gamma} .
$$

Assume that $\varphi \in \varphi_{\lambda} \triangleleft \mathbb{U}_{\tilde{\pi}}$, a subspace of $V(\lambda)^{*}$. Then we have $\alpha \cdot \omega_{1}=\beta \cdot \omega_{1}=0$. Thus $\kappa\left(c_{\varphi, v}\right) \in \mathbb{U}_{\tilde{\pi}}$. Finally, assume also that $\lambda \cdot \omega_{1}=0$. Then $\kappa\left(c_{\varphi, v}\right) \in \mathbb{U}_{\pi}^{\prime}$.

Now we fix $\lambda=\omega_{n-1}-\omega_{n}$. We have $\iota\left(c_{i j}\right)=c_{v_{j}, \varphi_{i}}$, an element of $\operatorname{cf}(V(\lambda))$. Equip $V\left(\omega_{1}\right)$ with the contragredient right action obtained by twisting the left action by the antipode. There is an isomorphism of right $\mathbb{U}$-modules $V(\lambda)^{*} \rightarrow V\left(\omega_{1}\right)$ such that $\varphi_{\lambda} \mapsto v_{n}$. If $j \neq 1$ the element $v_{j} \in V\left(\omega_{1}\right)$ belongs to $\iota\left(\mathbb{U}_{\tilde{\pi}}\right) \triangleright v_{n}$. Hence $\kappa\left(c_{v_{j}, \varphi_{i}}\right) \in \mathbb{U}_{\pi}^{\prime}$ for all $i$ by the remark above.

(b) Recall that $\left(\operatorname{ad}_{r} u\right) f=\iota\left(u_{2}\right) \triangleright f \triangleleft u_{1}$ for $f \in \mathbb{F}, u \in \mathbb{U}$. The natural pairing $\mathbb{F} \times \mathbb{U}^{\prime} \rightarrow \mathcal{K}$ is nondegenerate and yields a duality between the right adjoint action on $\mathbb{F}$ and the left adjoint action on $\mathbb{U}^{\prime}$, i.e., we have

$$
\left(\left(\operatorname{ad}_{r} u\right) f: u^{\prime}\right)=\left(f:(\operatorname{ad} u) u^{\prime}\right), \quad \forall f, u, u^{\prime} .
$$

The subspace $\mathbb{F}^{\pi} \subset \mathbb{F}$ is orthogonal to $\mathbb{I}_{V}$. The natural pairing $\mathbb{F}^{\pi} \times \mathbb{V} \rightarrow \mathcal{K}$ is nondegenerate. Hence $\mathbb{V}$ embeds into $\left(\mathbb{F}^{\pi}\right)^{*}$. A direct computation shows that the 
right adjoint action and the right contragredient action of $\iota\left(\mathbb{U}_{\pi}\right)$ on $\mathbb{F}^{\pi}$ are the same. Thus we have

$$
(\iota(u) \triangleright f: v)=(f:(\operatorname{ad} u) v), \quad \forall f \in \mathbb{F}^{\pi}, u \in \iota\left(\mathbb{U}_{\pi}\right), v \in \mathbb{V} .
$$

Now, recall notation 1.7.3(c). For each $\mu=\left(\mu_{1}, \mu_{2}, \ldots, \mu_{n}\right)$, a tuple of integers $\geqslant 0$, we set also $c_{\mu 1}=\left(c_{11}\right)^{\mu_{1}}\left(c_{21}\right)^{\mu_{2}} \cdots\left(c_{n 1}\right)^{\mu_{n}}$. Let $[r] ! \in \mathcal{K}$ be the $q$-analogue of $r$ !. Put $[\mu]$ ! $=\prod_{i=1}^{n}\left[\mu_{i}\right]$ !. The pairing

$$
\mathbb{F}^{\pi} \times{ }^{\pi} \mathbb{F} \rightarrow \mathcal{K}, \quad\left(c_{1 \lambda}, c_{\mu 1}\right) \mapsto\left\langle c_{1 \lambda}: c_{\mu 1}\right\rangle=\delta_{\lambda \mu}[\lambda] !
$$

is nondegenerate. Thus we may view the $\mathcal{K}$-vector space $\left(\mathbb{F}^{\pi}\right)^{*}$ as a completion of $\pi \mathbb{F}$. Furthermore, we have

$$
\left\langle\iota(u) \triangleright c_{1 \lambda}: c_{\mu 1}\right\rangle=\left\langle c_{1 \lambda}: c_{\mu 1} \triangleleft \iota(u)\right\rangle, \quad \forall u \in \iota\left(\mathbb{U}_{\pi}\right) .
$$

Thus the adjoint representation of $\iota\left(\mathbb{U}_{\pi}\right)$ on $\mathbb{V}$ embeds in a completion of the contragredient left $\iota\left(\mathbb{U}_{\pi}\right)$-module ${ }^{\pi} \mathbb{F}$.

By the PBW theorem the $\mathcal{K}$-vector space $\mathbb{V}=\mathbb{U}^{\prime} / \mathbb{I}_{V}$ is spanned by the classes of the elements of the form $k_{r \varepsilon_{1}} u_{+}$where $r \in 2 \mathbb{Z}$ and $u_{+} \in \mathbb{U}_{+}$is a product of quantum root vectors associated with the $\operatorname{roots} \varepsilon_{1}-\varepsilon_{2}, \varepsilon_{1}-\varepsilon_{3}, \ldots \varepsilon_{1}-\varepsilon_{n}$. The image of $\mathbb{V}$ in the completion of ${ }^{\pi} \mathbb{F}$ is computable. It is spanned by the formal sums $\exp _{q}\left(q^{r} c_{11}\right) c_{\mu 1}$ with $r \in 2 \mathbb{Z}$ and $\mu_{1}=0$ (left to the reader). Here we have set $\exp _{q}(a)=\sum_{r \geqslant 0} a^{r} /[r]$ !, as usual. Thus the $\mathcal{K}$-linear map

$$
{ }^{\pi} \mathbb{F} \rightarrow \mathbb{V}, \quad c_{\lambda 1} \mapsto \exp _{q}\left(q^{-\lambda_{1}} c_{11}\right) c_{\lambda 1}
$$

is an inclusion of $\iota\left(\mathbb{U}_{\pi}\right)$-modules ${ }^{\pi} \mathbb{F} \subset \mathbb{V}$ which maps onto $\mathbb{V}_{+}$.

A.4.1. Lemma. Let $f \in \operatorname{cf}(V(\lambda))$ with $\lambda \in X_{+}$. If we have $k_{\mu} \triangleright f \triangleleft k_{\nu}=$ $q^{\mu \cdot(\lambda-\gamma)+\nu \cdot(\lambda-\alpha)} f$ for each $\lambda, \mu$, then

$$
\kappa(f) \in \bigoplus_{\beta \in Y_{+}}\left(\mathbb{U}_{+}\right)_{\alpha-\beta} k_{2 \lambda-2 \beta} \iota\left(\mathbb{U}_{-}\right)_{\beta-\gamma}, \quad \beta \leqslant \alpha, \gamma
$$

Proof. There is a nondegenerate pairing $\langle:\rangle: \mathbb{U} \times \mathbb{U} \rightarrow \mathcal{K}$ such that

$$
\langle\kappa(f): u\rangle=(f: u), \quad\left\langle(\operatorname{ad} u)\left(u^{\prime}\right): u^{\prime \prime}\right\rangle=\left\langle u^{\prime}:\left(\operatorname{ad}_{r} u\right)\left(u^{\prime \prime}\right)\right\rangle ;
$$

see [T1, 2.2.1]. Now, we have

$$
\kappa \mathrm{cf}(V(\lambda)) \subset \bigoplus_{\beta \in Y_{+}} \mathbb{U}_{+} k_{2 \lambda-2 \beta} \iota\left(\mathbb{U}_{-}\right) .
$$

Let $f \in \operatorname{cf}(V(\lambda))$ be such that $\kappa(f)=u_{+} k_{2 \lambda-2 \beta} \iota\left(u_{-}\right)$with $u_{+} \in\left(\mathbb{U}_{+}\right)_{\alpha-\beta}$ and $u_{-} \in\left(\mathbb{U}_{-}\right)_{\beta-\gamma}$. Since $\left\langle\kappa(f): u^{\prime}\right\rangle=\left(f: u^{\prime}\right)$ we have

$$
\left\langle\kappa\left(f \triangleleft k_{\nu}\right): u^{\prime}\right\rangle=\left\langle\kappa(f): k_{\nu} u^{\prime}\right\rangle, \quad\left\langle\kappa\left(k_{\mu} \triangleright f\right): u^{\prime}\right\rangle=\left\langle\kappa(f): u^{\prime} k_{\mu}\right\rangle .
$$

Furthermore, [T1, 2.2.2] yields

$$
\left\langle\kappa(f): u_{-}^{\prime} k_{\lambda^{\prime}} \iota\left(u_{+}^{\prime}\right)\right\rangle=\left\langle u_{+}: u_{-}^{\prime}\right\rangle\left\langle u_{+}^{\prime}: u_{-}\right\rangle q^{(\lambda-\beta) \cdot \lambda^{\prime}} .
$$

So a direct computation yields

$$
\left\langle\kappa\left(f \triangleleft k_{\nu}\right): u^{\prime}\right\rangle=q^{\nu \cdot(\lambda-\alpha)}\left\langle\kappa(f): u^{\prime}\right\rangle, \quad\left\langle\kappa\left(k_{\mu} \triangleright f\right): u^{\prime}\right\rangle=q^{\mu \cdot(\lambda-\gamma)}\left\langle\kappa(f): u^{\prime}\right\rangle .
$$




\section{A.5. Proof of 1.8 .}

Proof of 1.8.2. (a) Use filtered/graded techniques as in the proof of 2.2.3( $a),(f)$.

(b) Use 1.4.3(c) and 1.7.3(d).

(c) Let $m$ denote the product in $\mathbb{D}$. The adjoint coaction $\operatorname{ad}^{*}: \mathbb{U}^{\prime} \rightarrow \mathbb{F} \otimes \mathbb{U}^{\prime}$ is an algebra homomorphism, because $\mathbb{U}^{\prime}$ is an (adU)-algebra. The QMM are given by $\partial_{\triangleleft}=m \circ\left(\ell \bar{\iota} \otimes \partial_{\triangleright}\right) \circ \mathrm{ad}^{*}$ and $\partial_{\triangleright}=m \circ\left(\ell \otimes \partial_{\triangleleft}\right) \circ \mathrm{ad}^{*}$, i.e., we have

$$
\partial_{\triangleleft} \kappa \iota(g)=\ell\left(\iota\left(g_{3}\right) g_{1}\right) \partial_{\triangleright} \kappa \iota\left(g_{2}\right), \quad \partial_{\triangleright} \kappa(f)=\ell\left(\iota\left(f_{1}\right) f_{3}\right) \partial_{\triangleleft} \kappa\left(f_{2}\right) .
$$

Let us check the second equality. The first one is proved in a similar way. We will regard $\mathbb{D}$ as the subalgebra of $\mathbb{D}_{\triangleleft}$ generated by $\ell(\mathbb{F})$ and $\partial_{\triangleleft}\left(\mathbb{U}^{\prime}\right)$. By part $(b)$ the basic representation of $\mathbb{D}_{\triangleleft}$ in $\mathbb{F}$ is faithful. Thus, by definition of a QMM, it is enough to check the relation

$$
\partial_{\triangleright}(u) \cdot f=u \triangleright f, \quad u \in \mathbb{U}^{\prime}, \quad f \in \mathbb{F} .
$$

Note that these relations imply, in particular, that $\partial_{\triangleright}$ is an algebra homomorphism. Now, the relation above is obtained in the following way. Write

$$
\operatorname{ad}^{*}(u)=\sum_{i} g_{i} \otimes u_{i}, \quad u, u_{i} \in \mathbb{U}^{\prime}, \quad g_{i} \in \mathbb{F} .
$$

Then we have

$$
\begin{gathered}
\sum_{i}\left(g_{i}: v\right) u_{i}=v_{1} u \iota\left(v_{2}\right), \quad v \in \mathbb{U}^{\prime}, \\
\partial_{\triangleright} u=\sum_{i} \ell\left(g_{i}\right) \partial_{\triangleleft}\left(u_{i}\right) .
\end{gathered}
$$

Let the second expression act on $\mathbb{F}$. We get

$$
\begin{aligned}
\left(\partial_{\triangleleft}(u) \cdot f: v\right) & =\sum_{i}\left(f_{1}: u_{i}\right)\left(g_{i} f_{2}: v\right) \\
& =\sum_{i}\left(f_{1}: u_{i}\right)\left(g_{i}: v_{1}\right)\left(f_{2}: v_{2}\right) \\
& =\left(f_{1}: v_{1} u \iota\left(v_{2}\right)\right)\left(f_{2}: v_{3}\right) \\
& =(f: v u) \\
& =(u \triangleright f: v) .
\end{aligned}
$$

$(d)$ Set $\psi=m \circ\left(\ell \otimes \partial_{\triangleleft}\right)$, a map $\mathbb{F} \otimes \mathbb{U}^{\prime} \rightarrow \mathbb{D}$. We have

$$
\sum_{i} \ell\left(f_{i}\right) \partial_{\triangleleft}\left(u_{i}\right)=0 \Rightarrow \sum_{i} f_{i}\left(f \triangleleft u_{i}\right)=0, \forall f \in \mathbb{F} .
$$

If $\sum_{i} f_{i} \otimes u_{i} \neq 0$ there is an element $f \in \mathbb{F}$ such that $\sum_{i} f_{i}\left(f \triangleleft u_{i}\right) \neq 0$. Thus $\psi$ is injective. The computation above yields $\psi \circ \mathrm{ad}^{*}=\partial_{\triangleright}$. Hence $\psi$ is surjective.

Proof of 1.8.3. (a) Recall that $\mathbb{D}_{\triangleright}^{\prime}=\mathbb{F}^{\prime} \sharp \mathbb{U}$ where $\mathbb{U}$ is identified with the normal left coideal subalgebra $\mathbb{U} \otimes 1 \subset \mathbb{U}^{[2]}$. Formula (A.2.1) yields an algebra isomorphism

$$
\Xi_{2}: \mathbb{D}_{\triangleright}^{\prime} \rightarrow \mathbb{D}_{\triangleleft}, \ell(f) \partial_{\triangleright}(u) \mapsto \sum_{s} \ell\left(\operatorname{ad} r_{s}^{+}\right)(f) \partial_{\triangleleft} \iota\left(r_{s}^{-} u\right) .
$$

We have $\left(\mathbb{U}^{\prime}\right)^{[2]} \subset \mathbb{U} \otimes \mathbb{U}^{\prime}$ by 1.7.1(a). Thus composing the map $\Xi_{2}^{-1}$ with the algebra homomorphism

$$
\mathbb{U} \otimes \mathbb{U}^{\prime} \rightarrow \mathbb{D}_{\triangleleft}, u \otimes v \mapsto \partial_{\triangleleft} \iota(u) \partial_{\triangleright}(v)
$$


yields an algebra homomorphism

$$
\partial_{2}:\left(\mathbb{U}^{\prime}\right)^{[2]} \rightarrow \mathbb{D}_{\triangleright}^{\prime} .
$$

Observe that $\Xi_{2}$ factors to the identity $\mathbb{F}^{\prime}=\mathbb{F}$ of the basic representations. So the $\mathbb{D}_{\triangleright}^{\prime}$-action on $\mathbb{F}^{\prime}$ is faithful. By definition of $\partial_{2}$ we have $\left(\partial_{2} u\right)(f)=u \triangleright f$ for each $u \in\left(\mathbb{U}^{\prime}\right)^{[2]}, f \in \mathbb{F}^{\prime}$. Thus $\partial_{2}$ is a QMM. Finally, $\partial_{2}$ maps into the subalgebra $\mathbb{D}^{\prime}=\mathbb{F}^{\prime} \sharp \mathbb{U}^{\prime}$ by formula A.5.3 below.

(b) We have $\Delta \kappa(f)=R^{-} \iota\left(f_{1}\right) R^{+}\left(f_{3}\right) \otimes \kappa\left(f_{2}\right)$ in $\mathbb{U}^{\otimes 2}$. Thus the coproduct of $\mathbb{U}^{[2]}$ yields

$$
\begin{aligned}
\Delta(\kappa(f) \otimes 1) & =R^{-} \iota\left(f_{1}\right) R^{+}\left(f_{3}\right) \otimes \iota\left(r_{s}^{+}\right) \otimes \kappa\left(\left(\operatorname{ad} r_{s}^{-}\right)\left(f_{2}\right)\right) \otimes 1, \\
& =R^{-} \iota\left(f_{1}\right) R^{+}\left(f_{5}\right) \otimes R^{-} \iota\left(f_{2}\right) R^{-}\left(f_{4}\right) \otimes \kappa\left(f_{3}\right) \otimes 1 .
\end{aligned}
$$

Therefore, in the ring $\mathbb{D}^{\prime}$ the following indentity holds:

$$
\partial_{\triangleright} \kappa(f) \ell\left(f^{\prime}\right)=\ell\left(\left(R^{-} \iota\left(f_{1}\right) R^{+}\left(f_{5}\right) \otimes R^{-} \iota\left(f_{2}\right) R^{-}\left(f_{4}\right)\right) \triangleright f^{\prime}\right) \partial_{\triangleright} \kappa\left(f_{3}\right) .
$$

This identity can be written in the following form:

$$
\partial_{\triangleright} \kappa\left(f_{2}\right) \ell\left(f^{\prime} \triangleleft R^{-} \iota\left(f_{1}\right) R^{+}\left(f_{3}\right)\right)=\ell\left(R^{-} \iota\left(f_{1}\right) R^{-}\left(f_{3}\right) \triangleright f^{\prime}\right) \partial_{\triangleright} \kappa\left(f_{2}\right) .
$$

Thus we have

$$
R_{21}^{q} M_{13} R_{12}^{q} M_{23}^{\prime}=M_{23}^{\prime} R_{21}^{q} M_{13}\left(R_{21}^{q}\right)^{-1}
$$

where $M=\sum_{i, j} e_{i j} \otimes \partial_{\triangleright} \kappa\left(c_{i j}\right)$ and $M^{\prime}=\sum_{i, j} e_{i j} \otimes \ell\left(c_{i j}\right)$. Therefore, by 1.7.1(b) the assignment $\ell_{i j} \mapsto \partial_{\triangleright} \kappa\left(c_{i j}\right)$ and $\ell_{i j}^{\prime} \mapsto \ell\left(c_{i j}\right)$ yields an embedding $\mathbb{D}_{+}^{\prime} \subset \mathbb{D}^{\prime}$. The elements $\ell_{\omega_{n}}, \ell_{\omega_{n}}^{\prime}$ may only quasi-commute with homogeneous elements of $\mathbb{D}_{+}^{\prime}$. Thus they generate a denominator set whose quotient ring is isomorphic to $\mathbb{D}^{\prime}$.

A.5.3. Lemma. For each $f, f^{\prime} \in \mathbb{F}$ we have the following formula in $\mathbb{D}^{\prime}$ :

$$
\partial_{2} \varpi_{2}\left(\kappa(f) \otimes \kappa\left(f^{\prime}\right)\right)=\sum_{s} \partial_{\triangleright} \kappa(f) \ell\left(\iota\left(r_{s}^{+}\right) \triangleright \iota\left(f_{1}^{\prime}\right) f_{3}^{\prime}\right) \partial_{\triangleright} \bar{\kappa}\left(\operatorname{ad} r_{s}^{-}\right) \bar{\iota}^{2}\left(f_{2}^{\prime}\right) .
$$

Proof. We will drop the summations symbol when there is no danger of confusion. First, observe that $\varpi_{2}\left(\kappa(f) \otimes \kappa\left(f^{\prime}\right)\right)=\varpi_{2}(\kappa(f) \otimes 1) \varpi_{2}\left(1 \otimes \kappa\left(f^{\prime}\right)\right)$. By definition of $\partial_{2}, \varpi_{2}$ we have

$$
\partial_{2} \varpi_{2}\left(\kappa(f) \otimes \kappa\left(f^{\prime}\right)\right)=\Xi_{2}^{-1} \partial_{\triangleleft} \iota\left(\kappa(f) r_{s}^{-}\right) \partial_{\triangleright} \kappa\left(\mathrm{ad} r_{s}^{+}\right)\left(f^{\prime}\right) .
$$

Thus $\partial_{2} \varpi_{2}(\kappa(f) \otimes 1)=\partial_{\triangleright} \kappa(f)$. Furthermore, (A.5.1) yields

$$
\begin{aligned}
\partial_{2} \varpi_{2}\left(1 \otimes \kappa\left(f^{\prime}\right)\right) & =\Xi_{2}^{-1} \ell\left(r_{s}^{+} \triangleright \iota\left(f_{1}^{\prime}\right) f_{3}^{\prime}\right) \partial_{\triangleleft} \kappa\left(f_{2}^{\prime}\right) \partial_{\triangleleft} \iota\left(r_{s}^{-}\right) \\
& =\ell\left(\operatorname{ad} r_{t}^{+}\right)\left(r_{s}^{+} \triangleright \iota\left(f_{1}^{\prime}\right) f_{3}^{\prime}\right) \partial_{\triangleright}\left(\bar{\iota}\left(r_{t}^{-}\right) \bar{\iota} \kappa\left(f_{2}^{\prime}\right) r_{s}^{-}\right) \\
& =\ell\left(r_{u}^{+} r_{s}^{+} \triangleright \iota\left(f_{1}^{\prime}\right) f_{3}^{\prime} \triangleleft \iota\left(r_{t}^{+}\right)\right) \partial_{\triangleright}\left(\bar{\iota}\left(r_{t}^{-} r_{u}^{-}\right) \bar{\iota} \kappa\left(f_{2}^{\prime}\right) r_{s}^{-}\right) \\
& =\ell\left(\iota\left(r_{s}^{+}\right) \triangleright \iota\left(f_{1}^{\prime}\right) f_{3}^{\prime} \triangleleft \iota\left(r_{t}^{+}\right)\right) \partial_{\triangleright}\left(\operatorname{ad} r_{s}^{-}\right) \bar{\iota}\left(\kappa\left(f_{2}^{\prime}\right) r_{t}^{-}\right) .
\end{aligned}
$$

Finally, a routine computation yields

$$
\ell\left(\iota\left(f_{1}^{\prime}\right) f_{3}^{\prime} \triangleleft \iota\left(r_{t}^{+}\right)\right) \partial_{\triangleright} \bar{\iota}\left(\kappa\left(f_{2}^{\prime}\right) r_{t}^{-}\right)=\ell\left(\iota\left(f_{1}^{\prime}\right) f_{3}^{\prime}\right) \partial_{\triangleright} \bar{\iota}^{2} \bar{\kappa}\left(f_{2}^{\prime}\right) .
$$

Proof of 1.8.4. The inclusion $\partial_{\triangleright}(\mathbb{U}) \subset\left(\mathbb{D}_{\triangleright}\right)^{\triangleleft \mathbb{U}}$ is obvious. Conversely, if the element $\sum_{i} \ell\left(f_{i}\right) \partial_{\triangleright}\left(u_{i}\right)$ lies in $\left(\mathbb{D}_{\triangleright}\right)^{\triangleleft \mathbb{U}}$ and the $u_{i}$ 's are linearly independent, then $f_{i}$ belongs to the subset $\mathbb{F}^{\triangleleft \mathbb{U}}$ for each $i$. As $\mathbb{F}^{\triangleleft \mathbb{U}}=\mathcal{K}$, this implies that $\partial_{\triangleright}(\mathbb{U})=\left(\mathbb{D}_{\triangleright}\right)^{\triangleleft \mathbb{U}}$. Finally, the relation $u \triangleright d=\partial_{\triangleright}\left(u_{1}\right) d \partial_{\triangleright} \iota\left(u_{2}\right)$ implies that $\partial_{\triangleright}(\mathbb{U})$ and $\left(\mathbb{D}_{\triangleright}\right)^{\mathbb{U} \triangleright}$ centralize each other. 


\section{A.6. Proof of 1.9 .}

Proof of 1.9.1. (a) The ring $\mathbb{R}_{\triangleright, *}^{\pi}$ is generated by $\ell\left(\mathbb{F}_{*}^{\pi}\right)$ and $\partial_{\triangleright}(\mathbb{U})$. It acts faithfully on $\mathbb{F}_{*}^{\pi}$ by [LR]. Therefore, it is a quantum torus by $1.7 .3(c)$. We have $\mathbb{R}_{\triangleright}^{\pi} \subset \mathbb{R}_{\triangleright, *}^{\pi}$ by [loc. cit.] Thus $\mathbb{R}_{\triangleright, *}^{\pi}$ and $\mathbb{R}_{\triangleright}^{\pi}$ are both ID.

(b) There is a nondegenerate pairing $\mathbb{F}^{\pi} \times \mathbb{V} \rightarrow \mathcal{K}$; see section A.4. Thus an element $d \in \mathbb{R}^{\pi}$ may be viewed as an element of $\mathbb{F}^{\pi} \otimes\left(\mathbb{F}^{\pi}\right)^{*}$ or, equivalently, as a linear operator $\mathbb{F}^{\pi} \rightarrow \mathbb{F}^{\pi}$. Let $d^{\prime \prime}$ be the natural action of $\mathbb{R}^{\pi}$ on $\mathbb{F}^{\pi}$. We must check that if $d^{\prime \prime}=0$, then $d^{\prime}=0$. This is proved as in 1.4.3(c).

Next we prove that $\mathbb{R}^{\pi}$ is an ID. Since $\mathbb{R}^{\pi} \subset \operatorname{End}\left(\mathbb{F}^{\pi}\right)$ we have also $\mathbb{R}_{i}^{\pi} \subset \operatorname{End}\left(\mathbb{F}_{i}^{\pi}\right)$ because for each $d \in \mathbb{R}^{\pi}$ we have

$$
c_{1 i}^{-m} d \cdot \mathbb{F}_{i}^{\pi}=0 \Rightarrow d \cdot \mathbb{F}^{\pi}=0 \Rightarrow d=0 \Rightarrow c_{1 i}^{-m} d=0 .
$$

A general definition of QDO algebra is given in [LR. The algebra $\mathbb{R}_{\triangleright}^{\pi}$ embeds into the QDO algebra of $\mathbb{F}^{\pi}$. Thus there are inclusions $\mathbb{R}_{\triangleright}^{\pi} \subset \mathbb{R}_{\triangleright, i}^{\pi} \subset \operatorname{End}\left(\mathbb{F}_{i}^{\pi}\right)$ by $[\mathrm{LR}$, 3.2.2, 3.3.5]. We have $\mathbb{R}_{i}^{\pi} \subset \mathbb{R}_{\triangleright, i}^{\pi}$ by A.6.3(c) below. Thus $\mathbb{R}_{i}^{\pi}$ is an ID by 1.9.1(a). Since $\mathbb{F} \subset \mathbb{F}_{i}$ we have $\mathbb{R}^{\pi} \subset\left(\mathbb{F}_{i} \otimes \mathbb{V}\right)^{\pi}=\mathbb{R}_{i}^{\pi}$. Thus $\mathbb{R}^{\pi}$ is also an ID.

Finally, we prove that $\mathbb{R}_{\Sigma}^{\pi}$ is a quantum torus. By A.6.3(c) below $\mathbb{R}_{\Sigma}^{\pi}$ is the $\mathcal{K}$ subalgebra of $\mathbb{R}_{\triangleright, *}^{\pi}$ generated by $\ell\left(\mathbb{F}_{*}^{\pi}\right)$ and $\partial_{\triangleright}\left(\mathbb{U}_{\Sigma}^{\prime}\right)$. Recall that $\mathbb{R}_{\triangleright, *}^{\pi}$ is isomorphic to the quantum torus generated by the elements $x_{i}^{ \pm 1}, y_{j}^{ \pm 1}$ in 1.7.3(c) by part $(a)$. By [J2, sec. 7.1.13] we have

$$
\mathbb{U}_{\Sigma}^{\prime}=\bigoplus_{\lambda \in 2 X} \mathbb{U}_{+} k_{\lambda} \iota\left(\mathbb{U}_{-}\right)
$$

Thus $\partial_{\triangleright}\left(\dot{e}_{j}\right), \partial_{\triangleright} \iota\left(\dot{f}_{j}\right)$ and $\partial_{\triangleright}\left(k_{\lambda}\right), \lambda \in 2 X$, belong to $\mathbb{R}_{\Sigma}^{\pi}$. Hence $\mathbb{R}_{\Sigma}^{\pi}$ contains the elements $x_{i}^{ \pm 1}, y_{i}^{ \pm 2}, y_{j}-y_{j}^{-1}, y_{j}^{-1}\left(y_{j+1}^{2}-1\right)$ for all $i, j$ with $j \neq n$. So $y_{j}^{ \pm 1} \in \mathbb{R}_{\Sigma}^{\pi}$ for all $n \neq j$. Thus $\mathbb{R}_{\Sigma}^{\pi}$ is the quantum torus generated by $x_{n}^{ \pm 1}, y_{n}^{ \pm 2}$ and $x_{j}^{ \pm 1}, y_{j}^{ \pm 1}$ with $n \neq j$.

(c) The map $\ell \otimes \partial_{\triangleleft}$ identifies $\mathbb{R}_{+}$with $\mathbb{F} \otimes \mathbb{V}_{+}$. The right $\mathbb{U}_{\pi}$-action and the left $\mathbb{U}$-action on $\mathbb{F} \otimes \mathbb{V}_{+}$are given by

$$
(f \otimes v) \triangleleft u=\left(f \triangleleft u_{1}\right) \otimes\left(\operatorname{ad} \bar{\imath} u_{2}\right)(v), \quad u \triangleright(f \otimes v)=(u \triangleright f) \otimes v .
$$

By 1.7.2(b) the $\mathcal{K}$-space $\mathbb{V}_{+}$, with the adjoint $\iota\left(\mathbb{U}_{\pi}\right)$-action, is isomorphic to ${ }^{\pi} \mathbb{F}$, with the contragredient left $\iota\left(\mathbb{U}_{\pi}\right)$-action. Thus $\mathbb{R}_{+}^{\pi} \simeq(\mathbb{F} \otimes \pi \mathbb{F})^{\pi}$ with

$$
\left(f \otimes f^{\prime}\right) \triangleleft u=\left(f \triangleleft u_{1}\right) \otimes\left(f^{\prime} \triangleleft u_{2}\right), \quad f \in \mathbb{F}, f^{\prime} \in \mathbb{F}^{\pi}, u \in \mathbb{U}_{\pi} .
$$

Now, identify the $(\mathbb{U}, \mathbb{U})$-bimodule $\mathbb{F}$ with $\bigoplus_{\lambda} V(\lambda) \otimes V(\lambda)^{*}$ and the right $\mathbb{U}$ module ${ }^{\pi} \mathbb{F}$ with $\bigoplus_{m} V\left(m \omega_{1}\right)^{*}$ in the obvious way. Frobenius reciprocity and the tensor identity yield the following chain of isomorphisms of left $\mathbb{U}$-modules

$$
\begin{aligned}
\left(\mathbb{F} \otimes{ }^{\pi} \mathbb{F}\right)^{\pi} & =\bigoplus_{\lambda, m} V(\lambda) \otimes\left(V(\lambda)^{*} \otimes V\left(m \omega_{1}\right)^{*}\right)^{\pi} \\
& =\bigoplus_{\lambda, m, m^{\prime}} V(\lambda) \otimes\left(V\left(m^{\prime} \omega_{1}\right) \otimes V\left(m \omega_{1}\right)^{\circ} \otimes V(\lambda)^{\circ}\right)^{\mathbb{U}} \\
& =\bigoplus_{m, m^{\prime}} V\left(m^{\prime} \omega_{1}\right) \otimes V\left(m \omega_{1}\right)^{\circ} \\
& =\mathbb{F}^{\pi} \otimes{ }^{\pi} \mathbb{F} .
\end{aligned}
$$


Under this sequence of isomorphisms the left $\mathbb{U}$-action on $\left(\mathbb{F} \otimes \otimes^{\pi} \mathbb{F}\right)^{\pi}$ given by the left $\mathbb{U}$-action on $\mathbb{F}$ is taken to the tensor product of the left $\mathbb{U}$-action on $\mathbb{F}^{\pi}$ and the contragredient left $\mathbb{U}$-action on ${ }^{\pi} \mathbb{F}$.

Given an algebra $\mathbf{A}$ and a Hopf algebra $\mathbf{H}$ which acts on $\mathbf{A}$ from the right let $\boldsymbol{M o d}_{r}(\mathbf{A}, \mathbf{H})$ be the category of right $\mathbf{H}$-equivariant left $\mathbf{A}$-modules. The Grothendieck category $\mathcal{M}_{\boldsymbol{M}}\left(\mathbb{F}, \mathbb{U}_{\tilde{\pi}}\right)$ is locally Noetherian because $\mathbb{F}$ is a Noetherian ring. For each integer $m$, tensoring a module $V \in \operatorname{Mod}_{r}\left(\mathbb{F}, \mathbb{U}_{\tilde{\pi}}\right)$ with the character

$$
[m]: \mathbb{U}_{\tilde{\pi}} \rightarrow \mathcal{K}, \quad u \mapsto\left(q^{m \omega_{1}}: u\right)
$$

yields a new object $V[m] \in \operatorname{Mod}_{r}\left(\mathbb{F}, \mathbb{U}_{\tilde{\pi}}\right)$. Here $\mathbb{F}$ acts on $V$ and $\mathbb{U}_{\tilde{\pi}}$ on the tensor product.

A.6.3. Lemma. (a) Let $V \rightarrow W$ be a surjective map of Noetherian objects in $\operatorname{Mod}_{r}\left(\mathbb{F}, \mathbb{U}_{\tilde{\pi}}\right)$. The induced map $V[m]^{\mathbb{U}_{\tilde{\pi}}} \rightarrow W[m]^{\mathbb{U}_{\tilde{\pi}}}$ is surjective if $m \gg 0$. The cokernel of the map $V^{\pi} \rightarrow W^{\pi}$ is a torsion module.

(b) The functor $\mathcal{M o d}_{r}^{l f}\left(\mathbb{U}_{\pi}\right) \rightarrow \operatorname{Mod}\left(\mathbb{F}_{i}^{\pi}\right), V \mapsto\left(\mathbb{F}_{i} \otimes V\right)^{\pi}$ is exact.

(c) The map $\mathbb{D}_{i}^{\pi} \rightarrow \mathbb{R}_{i}^{\pi}$ is surjective.

(d) The ring $\mathbb{R}_{i}^{\pi}$ is isomorphic to the subring of $\mathbb{R}_{\triangleright, i}^{\pi}$ generated by $\mathbb{D}_{i}^{\pi}$.

Proof. (a) Follows from Kempf's vanishing theorem in APW as in BK3, 3.4-3.5].

(b) The ring $\mathbb{F}^{\pi}$ is $\mathbb{Z}_{+}$-graded; see $1.7 .3(b)$. Let $\mathcal{Q} \boldsymbol{g r}\left(\mathbb{F}^{\pi}\right)$ be the quotient of $\mathcal{G r}\left(\mathbb{F}^{\pi}\right)$ by the subcategory consisting of torsion modules. If $V \in \mathcal{G r}\left(\mathbb{F}^{\pi}\right)$ is a torsion module, then $V_{i}=0$. So we have a commutative diagram

$$
\begin{array}{ccccc}
\operatorname{Mod}_{r}^{\mathrm{lf}}\left(\mathbb{U}_{\tilde{\pi}}\right) & \stackrel{a}{\rightarrow} & \operatorname{Mod}_{r}\left(\mathbb{F}, \mathbb{U}_{\tilde{\pi}}\right) & \stackrel{b}{\rightarrow} & \operatorname{Mod}_{r}\left(\mathbb{F}_{i}, \mathbb{U}_{\tilde{\pi}}\right) \\
& d \downarrow & & \downarrow \\
& \mathcal{Q g r}\left(\mathbb{F}^{\pi}\right) & \stackrel{e}{\rightarrow} & \operatorname{Mod}\left(\mathbb{F}_{i}^{\pi}\right),
\end{array}
$$

where $a(V)=\mathbb{F} \otimes V, b(V)=e(V)=V_{i}$ and $c(V)=d(V)=V^{\pi}=\bigoplus_{m \in \mathbb{Z}} V\langle m\rangle^{\mathbb{U}_{\tilde{\pi}}}$. Hence $d$ is exact by part $(a)$. Thus $c b a=e d a$ is an exact functor

$$
\mathcal{M o d}_{r}^{\mathrm{lf}}\left(\mathbb{U}_{\tilde{\pi}}\right) \rightarrow \mathcal{M o d}^{\mathrm{lf}}\left(\mathbb{F}_{i}^{\pi}\right) .
$$

$(c),(d)$ The projection $\mathbb{D}_{i} \rightarrow \mathbb{R}_{i}$ factors to $\mathbb{D}_{i}^{\pi} \rightarrow \mathbb{R}_{i}^{\pi}$. Observe that $\mathbb{D}_{i}=\mathbb{F}_{i} \otimes \mathbb{U}^{\prime}$, $\mathbb{R}_{i}=\mathbb{F}_{i} \otimes \mathbb{V}$ and that the map $\mathbb{D}_{i} \rightarrow \mathbb{R}_{i}$ is induced by the obvious projection $\mathbb{U}^{\prime} \rightarrow \mathbb{V}$. Thus $(c)$ follows from $(b)$. Part $(d)$ is obvious.

\section{A.7. Proof of 1.10 .}

Proof of 1.10.1. (a) By 1.3.2(b) we have

$$
\Delta\left(v \otimes 1^{2}\right)=\sum_{s, t} v_{1} \otimes \iota\left(r_{s}^{+}\right) \otimes \iota\left(r_{t}^{+}\right) \otimes\left(\operatorname{ad} r_{t}^{-} r_{s}^{-}\right)\left(v_{2}\right) \otimes 1^{2}
$$

in $\mathbb{U}^{[3]}$. Thus $\partial_{\triangleright}(v \otimes 1) \ell(f \otimes 1)=\ell\left(\left(v_{1} \triangleright f\right) \otimes 1\right) \partial_{\triangleright}\left(v_{2} \otimes 1\right)$ in $\mathbb{E}$. Hence $\gamma$ is an algebra homomorphism, because $\partial_{\triangleright}(v) \ell(f)=\ell\left(v_{1} \triangleright f\right) \partial_{\triangleright}\left(v_{2}\right)$ in $\mathbb{D}$.

From 1.3.2(b) we also have the following formula in $\mathbb{E}$ :

$$
\begin{array}{r}
\partial_{\triangleright}\left(v \otimes u^{\prime}\right) \ell\left(f \otimes f^{\prime}\right)=\ell\left(\left(v_{1} \triangleright f\right) \otimes \iota\left(r_{z}^{+} r_{y}^{+} r_{x}^{+}\right) \triangleright f^{\prime} \triangleleft \iota\left(\iota\left(r_{s}^{+}\right) u_{1}^{\prime} r_{t}^{+}\right)\right) . \\
\cdot \partial_{\triangleright}\left(r_{x}^{-} r_{s}^{-} v_{2} r_{t}^{-} \iota\left(r_{z}^{-}\right) \otimes\left(\operatorname{ad} r_{y}^{-}\right)\left(u_{2}^{\prime}\right)\right) .
\end{array}
$$


Here the summation symbol is omitted. Thus

$$
\begin{aligned}
\partial_{\triangleright} \varpi_{2}\left(1 \otimes u^{\prime}\right) \ell\left(1 \otimes f^{\prime}\right)= & \ell\left(1 \otimes\left(\iota\left(r_{z}^{+} r_{y}^{+} r_{x}^{+}\right) \triangleright f^{\prime} \triangleleft \iota\left(u_{1}^{\prime}\right)\right)\right) . \\
& \cdot \partial_{\triangleright}\left(r_{x}^{-} r_{b}^{-} \iota\left(r_{z}^{-}\right) \otimes\left(\operatorname{ad} r_{y}^{-} r_{b}^{+}\right)\left(u_{2}^{\prime}\right)\right) \\
= & \ell\left(1 \otimes\left(\iota\left(r_{x}^{+}\right) \triangleright f^{\prime} \triangleleft \iota\left(u_{1}^{\prime}\right)\right)\right) \partial_{\triangleright}\left(\mathrm{ad}^{[2]} \Delta r_{x}^{-}\right) \varpi_{2}\left(1 \otimes u_{2}^{\prime}\right) \\
= & \ell\left(1 \otimes\left(\iota\left(r_{x}^{+}\right) \triangleright f^{\prime} \triangleleft \iota\left(u_{1}^{\prime}\right)\right)\right) \partial_{\triangleright} \varpi_{2}\left(1 \otimes\left(\operatorname{ad} r_{x}^{-}\right)\left(u_{2}^{\prime}\right)\right) .
\end{aligned}
$$

By 1.3.2(a) we have

$$
\partial_{\triangleright}\left(u^{\prime}\right) \ell\left(f^{\prime}\right)=\sum_{s} \ell\left(\iota\left(r_{s}^{+}\right) \triangleright f^{\prime} \triangleleft \iota\left(u_{1}^{\prime}\right)\right) \partial_{\triangleright}\left(\mathrm{ad} r_{s}^{-}\right)\left(u_{2}^{\prime}\right)
$$

in $\mathbb{D}^{\prime}$. Thus $\gamma^{\prime}$ is an algebra homomorphism.

(b) By (A.7.1) the following formula holds in $\mathbb{E}$ :

$$
\partial_{\triangleright}(v \otimes 1) \ell\left(1 \otimes f^{\prime}\right)=\sum_{s} \ell\left(1 \otimes\left(\operatorname{ad} \iota r_{s}^{+}\right)\left(f^{\prime}\right)\right) \partial_{\triangleright}\left(\left(\operatorname{ad} r_{s}^{-}\right)(v) \otimes 1\right) .
$$

Setting $d=\ell(f) \partial_{\triangleright}(v)$ and $d^{\prime}=\ell\left(f^{\prime}\right) \partial_{\triangleright}\left(u^{\prime}\right)$ yields

$$
\psi\left(d \otimes d^{\prime}\right)=\sum_{s} \ell\left(f \otimes\left(\operatorname{ad} \iota r_{s}^{+}\right)\left(f^{\prime}\right)\right) \partial_{\triangleright} \varpi_{2}\left(\left(\operatorname{ad} r_{s}^{-}\right)(v) \otimes u^{\prime}\right) .
$$

So $\psi$ is invertible.

(c) A routine computation yields

$$
\begin{aligned}
\left(u \otimes 1^{3}\right) \triangleright \psi\left(d \otimes d^{\prime}\right) & =\psi\left((d \triangleleft \iota(u)) \otimes d^{\prime}\right), \\
\left(1 \otimes \Delta^{2}(u)\right) & \triangleright \psi\left(d \otimes d^{\prime}\right) \\
& =\psi\left(\ell\left(u_{1} \triangleright f\right) \partial_{\triangleright}\left(\operatorname{ad} u_{2}\right)(v) \otimes\left(\operatorname{ad} u_{3}\right)\left(d^{\prime}\right)\right) .
\end{aligned}
$$

(d) The basic $\mathbb{D}_{\triangleright}$-module is faithful by 1.8.2(b). The basic representation of $\mathbb{D}_{\triangleright}^{\prime}$ on $\mathbb{F}^{\prime}$ is also faithful by 1.4.3(b). Let $m, m^{\prime}$ be the multiplication in $\mathbb{F}, \mathbb{F}^{\prime}$, respectively. The multiplication of $f \otimes f^{\prime}, g \otimes g^{\prime} \in \mathbb{G}$ is given by

$$
\sum_{s} m\left(f, r_{s}^{-} \triangleright g\right) \otimes m^{\prime}\left(\left(\operatorname{ad} r_{s}^{+}\right)\left(f^{\prime}\right), g^{\prime}\right) .
$$

So (A.2.1) yields an algebra isomorphism

$$
\begin{aligned}
\Xi_{3}: \mathbb{G} \sharp \mathbb{U}^{[2]} & \rightarrow \mathbb{D}_{\triangleright} \otimes \mathbb{D}_{\triangleright}^{\prime}, \\
\ell\left(f \otimes f^{\prime}\right) \partial_{\triangleright}\left(v \otimes u^{\prime}\right) & \mapsto \sum_{s} \ell(f) \partial_{\triangleright}\left(r_{s}^{-} v\right) \otimes \ell\left(\operatorname{ad} r_{s}^{+}\right)\left(f^{\prime}\right) \partial_{\triangleright}\left(u^{\prime}\right) .
\end{aligned}
$$

Thus the basic representation of $\mathbb{G} \sharp \mathbb{U}^{[2]}$ on $\mathbb{G}$ is faithful. Hence the basic representation of the subalgebra $\mathbb{E} \subset \mathbb{G} \sharp \mathbb{U}^{[2]}$ on $\mathbb{G}$ is also faithful.

(e) By 1.8.2(c), 1.8.3(a) there is a QMM,

$$
\iota\left(\mathbb{U}^{\prime}\right) \otimes \mathbb{U} \otimes\left(\mathbb{U}^{\prime}\right)^{[2]} \rightarrow \mathbb{D}_{\triangleright} \otimes \mathbb{D}^{\prime}, \quad u \otimes v \otimes u^{\prime} \otimes v^{\prime} \mapsto \partial_{\triangleleft} \iota(u) \partial_{\triangleright}(v) \otimes \partial_{2}\left(u^{\prime} \otimes v^{\prime}\right) .
$$

Note that $\mathbb{H}^{\prime}$ is contained in the left-hand side by 1.7.1(a). Thus, composing this map with the isomorphism $\Xi_{3}^{-1}$ above we get an homomorphism

$$
\partial_{3}: \mathbb{H}^{\prime} \rightarrow \mathbb{G} \sharp \mathbb{U}^{[2]} .
$$

It is a QMM by part $(a)$ and 1.5.3(a). It maps into $\mathbb{E}$ by A.7.4( $a$ ) below. 
Proof of 1.10.3. (a) Obvious.

(b) The algebra isomorphisms (A.5.2), (A.7.2)

$$
\Xi_{2}: \mathbb{D}_{\triangleright}^{\prime} \rightarrow \mathbb{D}_{\triangleleft}, \quad \Xi_{3}: \mathbb{G}_{\sharp} \mathbb{U}^{[2]} \rightarrow \mathbb{D}_{\triangleright} \otimes \mathbb{D}_{\triangleright}^{\prime}
$$

yield an algebra embedding

$$
\Xi=\left(1 \otimes \Xi_{2}\right) \circ \Xi_{3}: \mathbb{E} \rightarrow \mathbb{D}_{\triangleright} \otimes \mathbb{D}_{\triangleleft} .
$$

It factors to an algebra embedding $\mathbb{S}^{\pi} \rightarrow \mathbb{R}_{\triangleright}^{\pi} \otimes \mathbb{D}_{\triangleleft}$. Recall that $\mathbb{D}_{\triangleleft}, \mathbb{R}_{\triangleright}^{\pi}$ are ID by 1.8.2(a), 1.9.1(a). Thus $\mathbb{S}^{\pi}$ is also an ID.

To conclude, we quote here more formulas for later use. Let $\partial_{d}$ denote the restriction of $\partial_{3}$ and the obvious embedding $\left(\mathbb{U}^{\prime}\right)^{[3]} \subset \mathbb{H}^{\prime}$.

A.7.4. Lemma. (a) We have $\partial_{d} \varpi_{3}\left(1 \otimes u^{\prime} \otimes v^{\prime}\right)=\gamma^{\prime} \partial_{2} \varpi_{2}\left(u^{\prime} \otimes v^{\prime}\right)$ and $\partial_{3}(u \otimes v$ $\left.\otimes 1^{2}\right)=\gamma\left(\partial_{\triangleleft} \iota(u) \partial_{\triangleright}(v)\right)$.

(b) We have $\partial_{b} \bar{\iota} \kappa(f)=\gamma\left(\partial_{\triangleleft} \kappa(f)\right)$ and $\partial_{c} \kappa(f)=\psi\left(\partial_{\triangleright} \kappa\left(f_{1}\right) \otimes \partial_{2} \Delta \kappa\left(f_{2}\right)\right)$.

(c) For $d=\ell(f) \partial_{\triangleright}(v), d^{\prime}=\ell\left(f^{\prime}\right) \partial_{\triangleright}\left(u^{\prime}\right)$ we have the following formula in $\mathbb{E}$

$$
\gamma^{\prime}\left(d^{\prime}\right) \gamma(d)=\sum_{x, y, z} \gamma\left(\ell\left(r_{x}^{-} \triangleright f\right) \partial_{\triangleright}\left(\operatorname{ad} r_{y}^{-} r_{z}^{-}\right)(v)\right) \gamma^{\prime}\left(\ell\left(\operatorname{ad} r_{y}^{+}\right)\left(f^{\prime}\right) \partial_{\triangleright}\left(\operatorname{ad} r_{z}^{+} r_{x}^{+}\right)\left(u^{\prime}\right)\right)
$$

(d) We have $\Xi \gamma^{\prime} \ell(f)=1 \otimes \ell(f)$ for each $f \in Z\left(\mathbb{F}^{\prime}\right)$.

(e) Fix a character $\chi \in X^{\prime}$. It extends uniquely to an algebra homomorphism $\chi: \mathbb{U} \rightarrow \mathcal{K}$. Let $\nu$ be the $\mathcal{K}$-algebra automorphism

$$
\nu: \mathbb{E} \rightarrow \mathbb{E}, \ell\left(f \otimes f^{\prime}\right) \partial_{\triangleright}\left(v \otimes u^{\prime}\right) \mapsto \ell\left(f \otimes f^{\prime}\right) \partial_{\triangleright}\left(v^{\chi} \otimes u^{\prime}\right) .
$$

For each $u \in \mathbb{U}^{\prime}$ we have $\nu \partial_{b} \bar{\iota}(u)=\partial_{b} \bar{\iota}\left(u^{\chi}\right)$ and $\nu \partial_{c}(u)=\partial_{c}\left(u^{\chi}\right)$.

Proof. (a) Before the proof let us quote the following formulas:

$$
\begin{gathered}
\Xi_{2} \circ \partial_{2}:\left(\mathbb{U}^{\prime}\right)^{[2]} \rightarrow \mathbb{D}_{\triangleleft}, u \otimes v \mapsto \partial_{\triangleleft} \iota(u) \partial_{\triangleright}(v), \\
\Xi \circ \partial_{3}: \mathbb{H}^{\prime} \rightarrow \mathbb{D}_{\triangleright} \otimes \mathbb{D}_{\triangleleft}, u \otimes v \otimes u^{\prime} \otimes v^{\prime} \mapsto \partial_{\triangleleft} \iota(u) \partial_{\triangleright}(v) \otimes \partial_{\triangleleft} \iota\left(u^{\prime}\right) \partial_{\triangleright}\left(v^{\prime}\right) .
\end{gathered}
$$

Now we can prove the claim. We have

$$
\partial_{3}\left(u \otimes v \otimes 1^{2}\right)=\Xi_{3}^{-1}\left(\partial_{\triangleleft} \iota(u) \partial_{\triangleright}(v) \otimes 1\right)=\gamma\left(\partial_{\triangleleft} \iota(u) \partial_{\triangleright}(v)\right) .
$$

Further, if $d^{\prime}=\ell\left(f^{\prime}\right) \partial_{\triangleright}\left(u^{\prime}\right)$, then

$$
\begin{aligned}
\Xi_{3} \gamma^{\prime}\left(d^{\prime}\right) & =\sum_{s, t} \partial_{\triangleright}\left(r_{s}^{-} r_{t}^{-}\right) \otimes \ell\left(\operatorname{ad} r_{s}^{+}\right)\left(f^{\prime}\right) \partial_{\triangleright}\left(\operatorname{ad} r_{t}^{+}\right)\left(u^{\prime}\right) \\
& =\sum_{s} \partial_{\triangleright}\left(r_{s}^{-}\right) \otimes\left(\operatorname{ad} r_{s}^{+}\right)\left(d^{\prime}\right) .
\end{aligned}
$$

Recall that $\partial_{2}$ is a QMM for the $\mathbb{U}^{[2]}$-action on $\mathbb{D}^{\prime}$. Thus we have

$$
(\operatorname{ad} u) \partial_{2}\left(u^{\prime} \otimes v^{\prime}\right)=\partial_{2}\left(\operatorname{ad}^{[2]} \Delta u\right)\left(u^{\prime} \otimes v^{\prime}\right) .
$$

Therefore (A.1.3) yields

$$
\begin{aligned}
\Xi_{3} \gamma^{\prime} \partial_{2} \varpi_{2}\left(u^{\prime} \otimes v^{\prime}\right) & =\sum_{s} \partial_{\triangleright}\left(r_{s}^{-}\right) \otimes \partial_{2}\left(\operatorname{ad}^{[2]} \Delta r_{s}^{+}\right) \varpi_{2}\left(u^{\prime} \otimes v^{\prime}\right) \\
& =\left(\partial_{\triangleright} \otimes \partial_{2}\right) \varpi_{3}\left(1 \otimes u^{\prime} \otimes v^{\prime}\right) \\
& =\Xi_{3} \partial_{d} \varpi_{3}\left(1 \otimes u^{\prime} \otimes v^{\prime}\right) .
\end{aligned}
$$

(b) Both claims follow from $(a)$ and 1.3.3(c), because $\Delta \kappa(f)=\varpi_{2}\left(\kappa f_{1} \otimes \kappa f_{2}\right)$. 
(c) It is enough to notice that

$$
\begin{aligned}
& \gamma^{\prime}\left(d^{\prime}\right) \gamma(d)=\sum_{s, t} \ell\left(\left(r_{s}^{-} \triangleright f\right) \otimes f^{\prime}\right) \partial_{\triangleright} \varpi_{2}\left(\left(\operatorname{ad} r_{t}^{-}\right)(v) \otimes\left(\operatorname{ad} r_{t}^{+} r_{s}^{+}\right)\left(u^{\prime}\right)\right), \\
& \gamma(d) \gamma^{\prime}\left(d^{\prime}\right)=\sum_{s} \ell\left(f \otimes\left(\operatorname{ad} \iota r_{s}^{+}\right)\left(f^{\prime}\right)\right) \partial_{\triangleright} \varpi_{2}\left(\left(\operatorname{ad} r_{s}^{-}\right)(v) \otimes u^{\prime}\right) .
\end{aligned}
$$

(d) By definition of $\gamma^{\prime}$ in section 1.10 we have $\Xi \gamma^{\prime} \ell(f)=\Xi \ell(1 \otimes f)$. Since $f \in Z\left(\mathbb{F}^{\prime}\right)$ formula (A.7.2) yields

$$
\begin{aligned}
\Xi \gamma^{\prime} \ell(f) & =\left(1 \otimes \Xi_{2}\right) \Xi_{3} \ell(1 \otimes f) \\
& =\sum_{s} \partial_{\triangleright}\left(r_{s}^{-}\right) \otimes \Xi_{2} \ell\left(\operatorname{ad} r_{s}^{+}\right)(f) \\
& =1 \otimes \Xi_{2} \ell(f) \\
& =1 \otimes \ell(f) .
\end{aligned}
$$

(e) By A.7.4(b) we have $\partial_{b} \bar{\iota}(u)=\gamma \partial_{\triangleleft}(u)$. Formula (A.1.1) and section 1.10 yield

$$
\begin{aligned}
\nu \gamma\left(\ell(f) \partial_{\triangleright}(v)\right) & =\ell(f \otimes 1) \partial_{\triangleright} \varpi_{2}(v \otimes 1)^{\chi \otimes \varepsilon} \\
& =\ell(f \otimes 1) \partial_{\triangleright}\left(v^{\chi} \otimes 1\right) \\
& =\gamma\left(\ell(f) \partial_{\triangleright}\left(v^{\chi}\right)\right) .
\end{aligned}
$$

The algebra homomorphism $\mathbb{D} \rightarrow \mathbb{D}, \ell(f) \partial_{\triangleright}(v) \mapsto \ell(f) \partial_{\triangleright}\left(v^{\chi}\right)$ takes $\partial_{\triangleleft}(u)$ to $\partial_{\triangleleft}\left(u^{\chi}\right)$. Thus we have

$$
\nu \partial_{b} \bar{\iota}(u)=\gamma \partial_{\triangleleft}\left(u^{\chi}\right)=\partial_{b} \bar{\iota}\left(u^{\chi}\right) .
$$

The second identity is left to the reader.

\section{A.8. Proof of 2.2.}

Proof of 2.2.5(b). Let $c=k / m \in \mathbb{Q}^{\times}$with $(m, k)=1$. Fix $a, b \in \mathbb{Z}$ such that $a k+b m=1$. We have

$$
\mathbb{Z}\left[q^{ \pm 1}, t^{ \pm 1}\right] /\left(q^{k}-t^{m}\right) \simeq \mathbb{Z}\left[u^{ \pm 1}\right], \quad q=u^{m}, \quad t=u^{k}, \quad u=q^{b} t^{a} .
$$

Let $(\tau, \zeta) \in \Gamma_{c}(\mathbb{C})$ with $\tau$ a root of unity of order $l=p^{e}$. Set $\varepsilon=\tau^{b} \zeta^{a}$, a generator of the subgroup of $\mathbb{C}^{\times}$generated by $\tau, \zeta$. Set $\mathrm{A}=\mathbb{Z}[\varepsilon] \subset \mathbb{C}$, a subring. There is a unique surjective ring homomorphism $\mathbb{Z}\left[u^{ \pm 1}\right] \rightarrow \mathrm{A}$ such that $u \mapsto \varepsilon$. It takes $q, t$ to $\tau, \zeta$, respectively. Let $h$ be the order of $\varepsilon$ and $\phi_{h} \in \mathbb{Z}[u]$ be the corresponding cyclotomic polynomial. We have $l=h /(m, h)$ because $\tau=\varepsilon^{m}$.

We claim that there is a surjective ring homomorphism $\mathrm{A} \rightarrow \mathrm{k}$ such that $\tau \mapsto 1$ and $\mathrm{k}$ is a finite field of characteristic $p$. Let $\mathrm{A}_{c}$ be the local ring of $\mathrm{A}$ at $\mathrm{k}$ and let $\mathrm{k}_{c}=\mathrm{k}$. Note that $\mathrm{A}$ is a Dedekind domain. Thus $\mathrm{A}_{c}$ is a DVR.

Now we prove the claim. Set $h=l^{\prime} m^{\prime}$ with $l^{\prime}$ a power of $p$ and $\left(m^{\prime}, p\right)=1$. Since $l=h /(m, h)$ is also a power of $p$ we have $m^{\prime} \mid m$. Let $\pi \in \mathbb{C}$ be a primitive $m^{\prime}$-th root of unity. Identify $\mathbb{Z}[u] /\left(\phi_{m^{\prime}}\right)$ with $\mathbb{Z}[\pi]$ so that $u \mapsto \pi$. Note that $u^{m} \mapsto 1$ because $m^{\prime} \mid m$. Set $\mathrm{k}_{c}=\mathbb{F}_{p}[\pi]$. Let $\mathbb{Z}[\pi] \rightarrow \mathrm{k}_{c}$ be the reduction modulo $p$. We claim that $\phi_{h} \mapsto 0$, yielding a surjective morphism $\mathrm{A} \rightarrow \mathrm{k}$ such that $\tau \mapsto 1$. To prove the claim it is enough to check that $p$ divides $\phi_{h}$ modulo $\phi_{m^{\prime}}$. Observe that in $\mathbb{C}[u]$ we 
have

$$
\begin{gathered}
\phi_{h}(u)=\phi_{m^{\prime}}\left(u^{l^{\prime}}\right) / \phi_{m^{\prime}}\left(u^{l^{\prime} / p}\right)=\prod_{i \in S} \sum_{j=0}^{p-1}\left(\pi^{i} u^{l^{\prime} / p}\right)^{j}, \\
S=\left\{i=1, \ldots, m^{\prime} ;\left(i, m^{\prime}\right)=1\right\} .
\end{gathered}
$$

The element $u^{l^{\prime} / p}$ maps to $\pi^{-i_{0}}$ for some $i_{0} \in S$, because $\left(p, m^{\prime}\right)=1$. Thus $\phi_{h}(u)$ maps to $\prod_{i} \sum_{j} \pi^{\left(i-i_{0}\right) j}$. We are done, because the $i_{0}$ th factor is equal to $p$.

Proof of 2.2.3. (a) The map $\kappa$ is an $\mathcal{A}$-algebra isomorphism $\mathbb{F}_{\mathcal{A}}^{\prime} \rightarrow \mathbb{U}_{\mathcal{A}}^{\prime}$ by definition of $\mathbb{U}_{\mathcal{A}}^{\prime}$. By [L2] we have

$$
R^{ \pm}\left(\mathbb{F}_{\mathcal{A}}\right) \subset \mathbb{U}_{\mathcal{A}}
$$

Thus $\mathbb{U}_{\mathcal{A}}^{\prime} \subset \mathbb{U}_{\mathcal{A}}$. We have $\left(\mathbb{F}_{\mathcal{A}}: \dot{U}_{\mathcal{A}}\right) \subset \mathcal{A}$ by DL. Thus we have

$$
\left(\operatorname{ad} \dot{U}_{\mathcal{A}}\right)\left(\mathbb{U}_{\mathcal{A}}^{\prime}\right) \subset \mathbb{U}_{\mathcal{A}}^{\prime}, \quad \dot{U}_{\mathcal{A}}^{e} \triangleright \mathbb{F}_{\mathcal{A}} \subset \mathbb{F}_{\mathcal{A}} .
$$

Thus $\mathbb{F}_{\mathcal{A}}, \mathbb{D}_{\mathcal{A}}$ are $\dot{\mathbb{U}}_{\mathcal{A}}^{e}$-algebras.

The isomorphism $\ell \otimes \partial_{\triangleright}: \mathbb{F} \otimes \mathbb{U} \rightarrow \mathbb{D}_{\triangleright}$ identifies $\mathbb{F}_{\mathcal{A}} \otimes \mathbb{U}_{\mathcal{A}}^{\prime}$ with $\mathbb{D}_{\mathcal{A}}$. Thus $\partial_{\triangleright}\left(\mathbb{U}_{\mathcal{A}}^{\prime}\right) \subset \mathbb{D}_{\mathcal{A}}$. By (A.5.1) we have $\partial_{\triangleleft} \kappa \iota(g)=\ell\left(\iota\left(g_{3}\right) g_{1}\right) \partial_{\triangleright} \kappa \iota\left(g_{2}\right)$ for each $g \in \mathbb{F}_{\mathcal{A}}$. Thus $\partial_{\triangleleft}\left(\mathbb{U}_{\mathcal{A}}^{\prime}\right) \subset \mathbb{D}_{\mathcal{A}}$.

The inclusion $\partial_{2}\left(\mathbb{U}_{\mathcal{A}}^{\prime}\right)^{[2]} \subset \mathbb{D}_{\mathcal{A}}$ follows from A.5.3, the inclusion $\psi\left(\mathbb{D}_{\mathcal{A}} \otimes \mathbb{D}_{\mathcal{A}}^{\prime}\right) \subset \mathbb{E}_{\mathcal{A}}$ from the definition of $\psi$ in section 1.10. The inclusion $\partial_{3}\left(\mathbb{H}_{\mathcal{A}}^{\prime}\right) \subset \mathbb{E}_{\mathcal{A}}$ is left to the reader.

Now we concentrate on the A-forms of the previous algebras. The A-algebras $\mathbb{U}_{\mathrm{A}}, \mathbb{F}_{\mathrm{A}}$ are NID by BG1, prop. 2.2, 2.7]. The proof that $\mathbb{F}_{\mathrm{A}}^{\prime}, \mathbb{U}_{\mathrm{A}}^{\prime}$ are NID is left to the reader. The proof that $\mathbb{D}_{\triangleright, \mathrm{A}}$ is a NID is standard, using filtered/graded technics. See part $(f)$ below.

Next, we must check that $\kappa$ yields an injection $\mathbb{F}_{\mathrm{A}}^{\prime} \rightarrow \mathbb{U}_{\mathrm{A}}$. Consider the quotient ring $\mathbb{U}_{\mathrm{A}, \Sigma}^{\prime}=\left(\mathbb{U}_{\mathrm{A}}^{\prime}\right)_{\Sigma}$. We have $\mathbb{U}_{\mathrm{A}}^{\prime} \subset \mathbb{U}_{\mathrm{A}, \Sigma}^{\prime}$, because $\mathbb{U}_{\mathrm{A}}^{\prime}$ is an ID. Thus it is enough to prove that the obvious map $\mathbb{U}_{\mathrm{A}, \Sigma}^{\prime} \rightarrow \mathbb{U}_{\mathrm{A}}$ is injective. To do that it is enough to prove that the $\mathcal{A}$-submodule $\mathbb{U}_{\mathcal{A}, \Sigma}^{\prime} \subset \mathbb{U}_{\mathcal{A}}$ is a direct summand. This follows from the following formula (compare (A.6.1)):

$$
\mathbb{U}_{\mathcal{A}, \Sigma}^{\prime}=\bigoplus_{m, n} \bigoplus_{\lambda \in 2 X} \mathcal{A} \dot{e}^{m} k_{\lambda} \iota\left(\dot{f}^{n}\right) .
$$

(b) The map $\kappa$ factors to an algebra isomorphism $\mathbf{F} \rightarrow \mathbf{U}^{\prime}$ by part (a). It yields an algebra isomorphism $\mathcal{F} \rightarrow \mathcal{U}^{\prime}$ by definition of $\mathcal{U}^{\prime}$. The subalgebra $\mathbf{u}^{e} \subset \mathbf{U}^{e}$ acts trivially on $\mathcal{F} \subset \mathbf{F}$. Thus $\dot{\mathbf{U}}^{e}$-action on $\mathbf{F}$ factors to a locally finite $\mathfrak{U}^{e}$-action on $\mathcal{F}$. Hence $\mathcal{F}$ is a $G^{2}$-algebra. Therefore $\mathcal{U}^{\prime}$ is a $G$-algebra for the adjoint action and the map $\kappa: \mathcal{U}^{\prime} \rightarrow \mathcal{F}$ commutes with the adjoint $G$-action.

The inclusion $\partial_{\triangleright}\left(\mathcal{U}^{\prime}\right) \subset \mathcal{D}$ is obvious. Given $u \in \mathbb{U}_{\mathcal{A}}^{\prime}$ we set $M=\left(\operatorname{ad} \dot{U}_{\mathcal{A}}\right)(u)$, a free $\mathcal{A}$-module of finite rank. The matrix coefficients of the adjoint representation of $\dot{U}_{\mathcal{A}}$ on $M$ belong to $\mathbb{F}_{\mathcal{A}}$. Thus we have $\operatorname{ad}^{*}\left(\mathbb{U}_{\mathcal{A}}^{\prime}\right) \subset \mathbb{F}_{\mathcal{A}} \otimes \mathbb{U}_{\mathcal{A}}^{\prime}$. This yields a map

$$
\mathrm{ad}^{*}: \mathbf{U}^{\prime} \rightarrow \mathbf{F} \otimes \mathbf{U}^{\prime} \text {. }
$$

This map factors to an algebra homomorphism $\mathcal{U}^{\prime} \rightarrow \mathcal{F} \otimes \mathcal{U}^{\prime}$, because $\mathcal{U}^{\prime}$ is a $G$-algebra for the adjoint action. Thus $\partial_{\triangleleft}\left(\mathcal{U}^{\prime}\right) \subset \mathcal{D}$ by (A.5.1).

The adjoint $\mathbf{u}$-action on $\mathcal{U}$ is trivial. Thus, to prove that $\mathcal{U}$ is a $G$-algebra for the adjoint action it is enough to prove that the adjoint $\dot{\mathbf{U}}$-action preserves $\mathcal{U}$. This is 
standard and is left to the reader. However, let us observe that if $\mathrm{A}$ is a field, then $\mathbb{U}_{\mathrm{A}}$ is an (adن் $\left.\dot{U}_{\mathrm{A}}\right)$-algebra, but if $\mathrm{A}$ is any ring this may be false.

The algebras $\mathbf{D}, \mathbf{D}_{\triangleright}$ are $\dot{\mathbf{U}}^{e}$-algebras; see section 1.8 . The subalgebra $\mathbf{u}^{e}$ acts trivially. So $\mathcal{D}, \mathcal{D}_{\triangleright}$ are $\mathfrak{U}^{e}$-algebra. Therefore $\mathcal{D}$ is a $G^{2}$-algebra.

(c) Assume initially that $\mathrm{A}=\mathbb{C}$ and $\mathcal{A}=\mathbb{C}\left[q^{ \pm 1}\right]$. Let $\mathfrak{z}_{U}: \mathcal{O}\left(G^{*}\right) \rightarrow \mathcal{U}$ be the isomorphism in [DKP, thm. 7.6], DP, sec. 19.1]. Fix $\bar{x}_{i}, \bar{y}_{i}, \bar{z}_{\lambda} \in \mathcal{O}\left(G^{*}\right)$ such that

$$
\mathfrak{z} U\left(\bar{x}_{i}\right)=x_{i}, \quad \mathfrak{z} U\left(\bar{y}_{i}\right)=y_{i}, \quad \mathfrak{z} U\left(\bar{z}_{\lambda}\right)=z_{\lambda} .
$$

By (2.6.7) there is an A-linear map $\nu: \mathcal{U} \rightarrow \dot{\mathbf{U}}$ such that

$$
(\operatorname{ad} \nu(u))(v)=\left\{u_{1}, v\right\} \iota\left(u_{2}\right), \quad v \in \mathcal{U} .
$$

Here $\{$,$\} is the Hayashi-Poisson bracket on \mathcal{U}$. Recall that

$$
\begin{gathered}
\iota\left(x_{i}\right)=-z_{i} x_{i}, \quad \iota\left(y_{i}\right)=-z_{i} y_{i}, \quad \iota\left(z_{\lambda}\right)=z_{\lambda}^{-1}, \\
\Delta\left(x_{i}\right)=z_{i}^{-1} \otimes x_{i}+x_{i} \otimes 1, \quad \Delta\left(y_{i}\right)=z_{i}^{-1} \otimes y_{i}+y_{i} \otimes 1, \quad \Delta\left(z_{\lambda}\right)=z_{\lambda} \otimes z_{\lambda} .
\end{gathered}
$$

A routine computation yields

$$
\nu\left(x_{i} z_{i}\right)=e_{i}^{(l)}, \quad \nu\left(y_{i} z_{i}\right)=-f_{i}^{(l)}, \quad \nu\left(z_{\lambda}\right)=h_{\lambda}^{(l)} / 2 .
$$

So we have

$$
\begin{aligned}
& \left(\operatorname{ad} e_{i}^{(l)}\right)(v)=\left\{x_{i} z_{i}, v\right\} z_{i}^{-1}, \\
& \left(\operatorname{ad} f_{i}^{(l)}\right)(v)=-\left\{y_{i} z_{i}, v\right\} z_{i}^{-1}, \quad \forall v \in \mathcal{U}, \\
& \left(\operatorname{ad} h_{\lambda}^{(l)}\right)(v)=2\left\{z_{\lambda}, v\right\} z_{\lambda}^{-1} .
\end{aligned}
$$

Now, consider the map $\theta$ in A.8.6(b) below. It is known that $\mathfrak{z} U$ is a Poisson-Hopf algebra homomorphism. From (A.8.3) and A.8.6(b) we get

$$
\begin{aligned}
& \left(\operatorname{ad} e_{i}^{(l)}\right) \circ \mathfrak{z}_{U}=\mathfrak{z}_{U} \circ \theta\left(\left(\bar{x}_{i} \bar{z}_{i}\right)^{\sharp}\right), \\
& \left(\operatorname{ad} f_{i}^{(l)}\right) \circ \mathfrak{z} U=-\mathfrak{z} U \circ \theta\left(\left(\bar{y}_{i} \bar{z}_{i}\right)^{\sharp}\right), \\
& \left(\operatorname{ad} h_{\lambda}^{(l)}\right) \circ \mathfrak{z} U=2 \mathfrak{z}_{U} \circ \theta\left(\left(\bar{z}_{\lambda}\right)^{\sharp}\right) .
\end{aligned}
$$

Next, let $\tilde{x}_{i}, \tilde{y}_{i}, \tilde{z}_{\lambda} \in \mathcal{O}\left(G^{*}\right)$ be as in [DKP sec. 7.5]. The computations in DKP, thm. 7.6], [DP, sec. 14.6] yield

$$
\tilde{x}_{i}^{\sharp}=-\bar{f}_{i}, \quad \tilde{y}_{i}^{\sharp}=\bar{e}_{i}, \quad \tilde{z}_{\lambda}^{\sharp}=-\bar{h}_{\lambda} / 2 .
$$

We have also

$$
\bar{x}_{i}=-\tilde{x}_{i} \tilde{z}_{i}, \quad \bar{y}_{i}=-\tilde{y}_{i} \tilde{z}_{i}, \quad \bar{z}_{\lambda}=\tilde{z}_{\lambda}^{-1}
$$

by $\left[\mathrm{DKP}\right.$, thm. 7.6], $\mathrm{DP}$, sec. 19.1]. Notice that our normalization for $x_{i}, y_{i}, z_{i}$ differs from that in [op. cit.]. Therefore A.8.6(b) yields

$$
\begin{aligned}
& \left(\operatorname{ad} e_{i}^{(l)}\right) \circ \mathfrak{z} U \circ \mathfrak{s}_{0}^{*}=\mathfrak{z} U \circ \theta\left(\bar{f}_{i}\right) \circ \mathfrak{s}_{0}^{*}=-\mathfrak{z} U \circ \mathfrak{s}_{0}^{*} \circ\left(\operatorname{ad} \bar{f}_{i}\right), \\
& \left(\operatorname{ad} f_{i}^{(l)}\right) \circ \mathfrak{z} U \circ \mathfrak{s}_{0}^{*}=\mathfrak{z} U \circ \theta\left(\bar{e}_{i}\right) \circ \mathfrak{s}_{0}^{*}=-\mathfrak{z} U \circ \mathfrak{s}_{0}^{*} \circ\left(\operatorname{ad} \bar{e}_{i}\right), \\
& \left(\operatorname{ad} h_{\lambda}^{(l)}\right) \circ \mathfrak{z}_{U} \circ \mathfrak{s}_{0}^{*}=\mathfrak{z} U \circ \theta\left(\bar{h}_{\lambda}\right) \circ \mathfrak{s}_{0}^{*}=-\mathfrak{z} U \circ \mathfrak{s}_{0}^{*} \circ\left(\operatorname{ad} \bar{h}_{\lambda}\right) .
\end{aligned}
$$

Here $\bar{e}_{i}, \bar{f}_{i}$ and $\bar{h}_{\lambda}$ are as in section 2.1. Thus we have

$$
(\operatorname{ad} u) \circ \mathfrak{z} U \circ \mathfrak{s}^{*}=\mathfrak{z} U \circ \mathfrak{s}^{*} \circ(\operatorname{ad} \phi(u)), \quad \forall u \in \dot{\mathbf{U}} .
$$

So the map $\mathfrak{z} U \circ \mathfrak{s}^{*}$ commutes with the adjoint $\mathfrak{U}$-action. 
The adjoint of the Frobenius homomorphism is a linear map $\mathfrak{U}^{*} \rightarrow \dot{\mathbf{U}}^{*}$. It factors to a map

$$
\mathfrak{z}_{F}: \mathcal{O}(G) \rightarrow \mathcal{F}
$$

which is a $G^{2}$-equivariant Hopf algebra isomorphism such that $\bar{c}_{i j} \mapsto\left(c_{i j}\right)^{l}$. See [DL, prop. 6.4] for details. Set

$$
\mathfrak{z} U^{\prime}=\kappa \circ \mathfrak{z}_{F}: \mathcal{O}(G) \rightarrow \mathcal{U}^{\prime} .
$$

The map $\mathfrak{z} U^{\prime}$ is a $(\operatorname{ad} G)$-equivariant algebra isomorphism. We have $\mathfrak{s}^{*}\left(\bar{c}_{\lambda}\right)=\bar{z}_{2 \lambda}$ for each $\lambda \in X_{+}$. Here $\bar{c}_{\lambda}$ is as in 1.7.3(e). Therefore we have also $\mathfrak{z} U \mathfrak{s}^{*}\left(\bar{c}_{\lambda}\right)=\mathfrak{z}_{U^{\prime}}\left(\bar{c}_{\lambda}\right)$. Since $\mathcal{O}(G)=\bigoplus_{\lambda \in X_{+}}(\operatorname{ad} \mathfrak{U})\left(\bar{c}_{\lambda}\right)$ we get $\mathfrak{z} U^{\prime}=\mathfrak{z} U \circ \mathfrak{s}^{*}$, because both maps commute with the adjoint $\mathfrak{U}$-action.

We define $\mathfrak{z}_{D}$ to be the $G^{2}$-algebra isomorphism

$$
\mathfrak{z}_{D}: \mathcal{O}(D)=\mathcal{O}(G) \otimes \mathcal{O}(G) \rightarrow \mathcal{D}, f \otimes f^{\prime} \mapsto \ell \mathfrak{z}_{F}(f) \partial_{\triangleleft \mathfrak{z} U^{\prime} \iota}\left(f^{\prime}\right) .
$$

Now, we consider more general rings A. In DL the map $\mathfrak{z}_{F}$ is defined over the field $\mathbb{Q}(\tau) \subset \mathbb{C}$. Since it is the unique algebra homomorphism such that $\bar{c}_{i j} \mapsto\left(c_{i j}\right)^{l}$, it is indeed defined over the subring $\mathbb{Z}[\tau]$. Here $\bar{c}_{i j}$ is as in 1.7.3(e). So there is an isomorphism $\mathcal{O}(G) \rightarrow \mathcal{F}$ for any A. Indeed, the surjectivity is obvious and injectivity follows from injectivity over $\mathbb{Q}(\tau)$ because $\mathcal{O}(G)$ is a torsion-free $\mathbb{Z}$ module. Thus $\mathfrak{z}_{F}, \mathfrak{z}_{U^{\prime}}, \mathfrak{z} D$ yield isomorphisms

$$
\mathcal{O}(G) \simeq \mathcal{F}, \quad \mathcal{O}(G) \simeq \mathcal{U}^{\prime}, \quad \mathcal{O}(D) \simeq \mathcal{D}
$$

for any A. A similar argument shows that $\mathfrak{z} U$ yields an isomorphism for all A:

$$
\mathcal{O}\left(G^{*}\right) \simeq \mathcal{U}
$$

$(d),(e),(f)$ It is well known that $\mathbf{U}$ is a free $\mathcal{U}$-module of rank $l^{n^{2}}$. For $\mathrm{A}=\mathbb{C}$ it is proved in $\mathrm{BGS}$ that $\mathbf{F}$ is a free $\mathcal{F}$-module of rank $l^{n^{2}}$. Recall that we have set $G=G L_{n}$. So $\mathbf{F}$ is a free $\mathcal{F}$-module of rank $l^{n^{2}}$ for any $\mathrm{A}$, because $\mathbf{F}$ is the quotient ring of $\mathbf{F}_{+}$. Finally $\mathbf{U}^{\prime}$ is a free $\mathcal{U}^{\prime}$-module, because $\kappa$ gives an isomorphism of $\mathcal{U}^{\prime}$-modules $\mathcal{U}^{\prime} \otimes_{\mathcal{F}} \mathbf{F} \rightarrow \mathbf{U}^{\prime}$.

Now, let $\mathcal{F}^{\prime} \subset \mathbf{F}^{\prime}$ be the image of $\mathcal{F}$ by the identity map $\mathbf{F} \rightarrow \mathbf{F}^{\prime}$. We have $\mathcal{D}=\ell(\mathcal{F}) \partial_{\triangleright}\left(\mathcal{U}^{\prime}\right)$, a central subalgebra of $\mathbf{D}$. Set $\mathcal{D}^{\prime}=\ell\left(\mathcal{F}^{\prime}\right) \partial_{\triangleright}\left(\mathcal{U}^{\prime}\right)$, a subalgebra of $\mathbf{D}^{\prime}$. Recall that $R^{ \pm}$maps $\mathbf{F}$ to $\mathbf{U}$; see (A.8.1). By [DL] we have also

$$
R^{ \pm}(\mathcal{F}) \subset \mathcal{U}
$$

Thus the twisting terms in the multiplication of $\mathbf{F}^{\prime}, \mathbf{D}^{\prime}$ vanish when restricted to $\mathcal{F}^{\prime}$, $\mathcal{D}^{\prime}$. So $\mathcal{F}^{\prime}, \mathcal{D}^{\prime}$ are central subalgebras of $\mathbf{F}^{\prime}, \mathbf{D}^{\prime}$. They are canonically isomorphic to $\mathcal{F}, \mathcal{D}$, respectively, as (adi்)-algebras. More precisely, we will identify the rings $\mathcal{D}, \mathcal{D}^{\prime}$ via the isomorphism

$$
\dagger: \mathcal{D} \rightarrow \mathcal{D}^{\prime}, \ell(f) \partial_{\triangleright}(u) \mapsto \ell \iota(f) \partial_{\triangleright}(u)
$$

Next, equip the algebra $\mathbf{D}_{\triangleright}$ with the filtration such that the element $\ell(g) \partial_{\triangleright}(u)$ with $u=\dot{e}^{m} k_{\lambda} \iota\left(\dot{f}^{n}\right)$ has the degree

$$
\left(n_{1}, n_{2}, \ldots, n_{N}, m_{N}, \ldots, m_{1}, \rho \cdot|m|+\rho \cdot|n|\right)=\left(\ldots, \operatorname{deg}_{2}, \operatorname{deg}_{1}\right) .
$$

The successive graded algebras associated to the partial degrees $\operatorname{deg}_{1}, \operatorname{deg}_{2}, \ldots$ form a finite sequence such that the first algebra is $\mathbf{D}_{\triangleright}$ and each term of the sequence is 
the associated graded ring of the previous one with respect to a positive filtration. We have

$$
\partial_{\triangleright}(u) \ell(f)=\ell\left(k_{\lambda-|m|-|n|} \triangleright f\right) \partial_{\triangleright}(u)+\text { lower terms for } \operatorname{deg}_{1} .
$$

Thus the last algebra in the sequence is an iterated twisted polynomial algebra over F. More precisely, it admits a positive filtration $F_{0} \subset F_{1} \subset \cdots$ such that $F_{0}=\mathbf{F}$ and $F_{i+1}=F_{i}\left[x_{i} ; \phi_{i}\right]$ for some algebra automorphism $\phi_{i}$ of $F_{i}$ of order $l$. See DP, sec. 10] for details.

Now, assume that $\mathrm{A}=\mathbb{C}$. Then $\mathbf{F}$ is a maximal order by $\mathrm{DL}$, sec. 7.4]. Thus $\mathbf{D}_{\triangleright}$ is also a maximal order by standard filtered/graded techniques (see A.10.1(b), VV1]). It is also a free $\mathcal{D}_{\triangleright}$-module of rank $l^{2 n^{2}}$. Hence the PI-degree of $\mathbf{D}_{\triangleright}$ is $\leqslant l^{n^{2}}$. It is equal to $l^{n^{2}}$ by A.8.6 $(d)$.

Let us prove that $Z(\mathbf{D})=\mathcal{D}$ and $Z\left(\mathbf{D}^{\prime}\right)=\mathcal{D}^{\prime}$. The center $Z\left(\mathbf{D}_{\triangleright}\right)$ is a direct summand in $\mathbf{D}_{\triangleright}$, because $\mathbf{D}_{\triangleright}$ is a maximal order. See section A.10 for details. Furthermore, $\mathbf{D}_{\triangleright}$ is a free $\mathcal{D}_{\triangleright}$-module of rank $l^{2 n^{2}}$ and its PI-degree is $l^{n^{2}}$. Thus $Z\left(\mathbf{D}_{\triangleright}\right)$ is a projective $\mathcal{D}_{\triangleright}$-module of rank one. So we have $\mathcal{D}_{\triangleright}=Z\left(\mathbf{D}_{\triangleright}\right)$. So we also have

$$
Z(\mathbf{D})=Z\left(\mathbf{D}_{\triangleright}\right) \cap \mathbf{D}=\mathcal{D} .
$$

The rest is proved in A.8.6(c).

A.8.6. Lemma. (a) The obvious projections $D^{*} \rightarrow G, G^{*}$ are Poisson homomorphisms. For $x, y \in T_{g^{*}}^{*} G^{*}$ and $\xi, \chi \in T_{g}^{*} G$ we have

$$
\begin{aligned}
\left((x+\xi) \otimes(y+\chi): \pi_{D^{*}}\right)= & \left(x \otimes y: \pi_{G^{*}}\right)+\left(\xi \otimes \chi: \pi_{G}\right)+\left\langle g^{-1} \triangleright \chi: x \triangleleft\left(g^{*}\right)^{-1}\right\rangle \\
& -\left\langle g^{-1} \triangleright \xi: y \triangleleft\left(g^{*}\right)^{-1}\right\rangle .
\end{aligned}
$$

(b) The right dressing action yields a Lie algebra homomorphism $\theta$ as follows:

$$
\theta: \mathfrak{g} \rightarrow \operatorname{Der}\left(\mathcal{O}\left(G^{*}\right)\right), \quad \theta\left(f^{\sharp}\right)\left(f^{\prime}\right)=\left\{f_{1}, f^{\prime}\right\} \iota\left(f_{2}\right), \quad \forall f, f^{\prime} \in \mathcal{O}\left(G^{*}\right) .
$$

The map $\mathfrak{s}_{0}: G^{*} \rightarrow G$ intertwines the right dressing action of $g$ on $G^{*}$ and the conjugation by $g^{-1}$ on $G$.

(c) Set $\mathrm{A}=\mathbb{C}$. There is a Poisson algebra isomorphism

$$
\mathfrak{m}: \mathcal{O}\left(D^{*}\right) \rightarrow \mathcal{D}_{\triangleright}, f \otimes f^{\prime} \mapsto \ell \mathfrak{z}_{F}(f) \partial_{\triangleright} \mathfrak{a}^{\prime} \mathfrak{z} U\left(f^{\prime}\right) .
$$

(d) Set $\mathbf{A}=\mathbb{C}$. The basic representation of $\mathbf{D}_{\triangleright}$ factors to a simple representation in $\mathbf{F} / \mathcal{F}^{\varepsilon} \mathbf{F}$. The algebra $\mathbf{D}_{\triangleright}$ is a Poisson order over $D^{*}$.

Proof of 2.2.4. By A.8.6(c) the map

$$
\mathfrak{m}: \mathcal{O}\left(D^{*}\right) \rightarrow \mathcal{D}_{\triangleleft}, f \otimes f^{\prime} \mapsto \ell \mathfrak{z}_{F}(f) \partial_{\triangleleft} \mathfrak{a}^{\prime} \mathfrak{z} U\left(f^{\prime}\right)
$$

is a Poisson algebra isomorphism. Furthermore, $\mathbf{D}_{\triangleleft}$ is a Poisson order over $D^{*}$ by A.8.6 $(d)$. Finally, the bivector field $\pi_{D^{*}}$ is nondegenerate over the open subset

$$
D_{\Sigma}^{*}=\left\{\left(g, g^{*}\right) \in D^{*} ; g g^{*} \in G^{*} G\right\}=\left\{\left(g, g^{*}\right) ; g \mathfrak{s}_{0}\left(g^{*}\right) g^{-1} \in G_{\Sigma}\right\} ;
$$

see [AM]. So the fibers of $\mathbf{D}_{\triangleleft}$ over $D_{\Sigma}^{*}$ are isomorphic as algebras by A.10.3 $(a)$.

By 2.2.3 $(f)$ the algebra $\mathbf{D}_{\triangleleft}$ is a prime PI-ring and $Z\left(\mathbf{D}_{\triangleleft}\right) \simeq \mathcal{O}\left(D^{*}\right)$. Thus there is a dense open subset in $D^{*}$ over which $\mathbf{D}_{\triangleleft}$ is an Azumaya algebra. See section A.10 for details. This open subset has a nonempty intersection with $D_{\Sigma}^{*}$. Therefore $\mathbf{D}_{\triangleleft}$ restricts to an Azumaya algebra over $D_{\Sigma}^{*}$. 
Set $\Sigma^{l}=\left\{k_{2 l \lambda} ; \lambda \in X_{+}\right\}$. Recall that $\mathfrak{z}_{D}: \mathcal{O}(D) \rightarrow \mathcal{D}$ is an isomorphism. By (A.5.1) we have

$$
\mathfrak{z}_{D}\left(\left\{1 \otimes \iota(f), \iota\left(f_{1}\right) f_{3} \otimes \iota\left(f_{2}\right) ; f \in \Sigma\right\}\right)=\partial_{\triangleright}\left(\Sigma^{l}\right) \cup \partial_{\triangleleft}\left(\Sigma^{l}\right) .
$$

So the corresponding quotient ring of $\mathcal{D}$ is isomorphic to $\mathcal{O}\left(D_{\Sigma}\right)$.

The multiplication in $\mathbf{U}$ gives an $\mathcal{U}$-algebra isomorphism $\mathcal{U} \otimes_{\mathcal{U}^{\prime}} \mathbf{U}^{\prime} \rightarrow \mathbf{U}$, because it is surjective and both sides are $\mathcal{U}$-free of $\operatorname{rank} l^{n^{2}}$. So the obvious inclusion $\mathcal{D} \subset \mathcal{D}_{\triangleleft}$ yields a $\mathcal{D}_{\triangleleft}$-algebra isomorphism

$$
\mathcal{D}_{\triangleleft} \otimes_{\mathcal{D}} \mathbf{D} \rightarrow \mathbf{D}_{\triangleleft} .
$$

Now, this inclusion fits into the commutative square

$$
\begin{array}{ccc}
\mathcal{D} & \rightarrow & \mathcal{D}_{\triangleleft} \\
\uparrow & & \uparrow \\
\mathcal{O}(D) & \rightarrow & \mathcal{O}\left(D^{*}\right) .
\end{array}
$$

Here the lower map is the comorphism of id $\times \mathfrak{s}_{0}: D^{*} \rightarrow D$ and the vertical maps are $\mathfrak{z}_{D}, \mathfrak{m}$. The commutativity of this square is a consequence of the equality

$$
\mathfrak{z} U^{\prime}=\mathfrak{a}^{\prime} \circ \mathfrak{z} U^{\prime} \circ\left(\mathfrak{a}^{\prime}\right)^{*}
$$

where $\mathfrak{a}^{\prime}$ is the anti-involution

$$
\mathfrak{a}^{\prime}: G \rightarrow G, g \mapsto{ }^{\mathrm{T}} g .
$$

Now the map id $\times \mathfrak{s}_{0}$ yields an étale cover $D_{\Sigma}^{*} \rightarrow D_{\Sigma}$. Thus $\mathbf{D}_{\Sigma}$ is an Azumaya algebra over $\mathcal{O}\left(D_{\Sigma}\right)$ by [M2, IV.2.1].

The case of $\mathbf{D}_{\Sigma}^{\prime}$ is similar. First, the map $\Xi_{2}$ yields an algebra isomorphism $\mathbf{D}_{\triangleright}^{\prime} \rightarrow \mathbf{D}_{\triangleleft}$ which takes $\mathcal{D}_{\triangleright}^{\prime}$ onto $\mathcal{D}_{\triangleleft}$. Thus $\mathbf{D}_{\triangleright}^{\prime}$ is an Azumaya algebra over $\mathcal{D}_{\triangleright}^{\prime}$. Further (A.5.1), A.5.3 yield

$$
\partial_{2} \varpi_{2}(\kappa f \otimes \kappa g)=\ell\left(\iota\left(g_{1}\right) g_{3}\right) \partial_{\triangleright} \kappa\left(\iota\left(g_{2}\right) f\right)=\dagger\left(\partial_{\triangleleft} \kappa \iota(g) \partial_{\triangleright} \kappa(f)\right) .
$$

Hence the isomorphism $\dagger \circ \mathfrak{z}_{D}: \mathcal{O}(D) \rightarrow \mathcal{D}^{\prime}$ identifies the localization of $\mathcal{D}^{\prime}$ at $\partial_{2} \varpi_{2}\left(\Sigma^{l} \otimes \Sigma^{l}\right)$ and the ring of functions $\mathcal{O}\left(D_{\Sigma}\right)$. The rest is as above.

Proof of A.8.6. (a) See [L3, secs. 5.2 and 5.3]. Note that ( : ) is the canonical duality pairing while $\langle:\rangle$ is the bilinear form (2.1.1).

(b) Let us recall the contsruction of $\theta$. For $x \in \mathfrak{g}$ let $x^{r}$ be the right invariant 1 -form on $G^{*}$ whose value at $e$ is $\sharp(x)$. There is a vector field $\theta^{\prime}(x)$ on $G^{*}$ such that

$$
\left(\omega: \theta^{\prime}(x)\right)=\left(x^{r} \wedge \omega: \pi_{G^{*}}\right), \quad \forall \omega \in \Omega_{G^{*}}^{1} .
$$

Compare [LW, def. 2.2]. Then $\theta(x)$ is the Lie derivative with respect to the vector field $\theta^{\prime}(x)$, acting from the left.

Now, we set $x=f^{\sharp}$. We have $x^{r}=\iota\left(f_{2}\right) \mathrm{d} f_{1}$ by [DP, sec. 14.6]. Hence

$$
\begin{aligned}
\theta\left(f^{\sharp}\right)\left(f^{\prime}\right) & =\theta^{\prime}\left(f^{\sharp}\right) \triangleright f^{\prime} \\
& =\left(\mathrm{d} f^{\prime}: \theta^{\prime}(x)\right) \\
& =\iota\left(f_{2}\right)\left(\mathrm{d} f_{1} \wedge \mathrm{d} f^{\prime}: \pi_{G^{*}}\right) \\
& =\left\{f_{1}, f^{\prime}\right\} \iota\left(f_{2}\right) .
\end{aligned}
$$

Next, observe that the right dressing action of $g \in G$ takes $g^{*} \in G^{*}$ to an element of $G^{*}$ whose image by the natural map

$$
G^{*} \subset D \rightarrow D / G
$$


is $g^{-1} g^{*} G / G$; see [LW, thm. 3.14]. Here $G$ embeds diagonally in $D$; see section 2.1 for details. The map $\mathfrak{s}_{0}: G^{*} \rightarrow G$ in (2.2.2) is the restriction of the map

$$
D \rightarrow G,\left(g, g^{\prime}\right) \mapsto g^{\prime} g^{-1}
$$

to $G^{*} \subset D$. The latter factors through a map $D / G \rightarrow G$ such that

$$
g^{-1} g^{*} G / G \mapsto g^{-1} \mathfrak{s}_{0}\left(g^{*}\right) g .
$$

Thus $\mathfrak{s}_{0}$ intertwines the right dressing action of $g$ on $G^{*}$ and the conjugation by $g^{-1}$ on $G$.

(c) Set $\mathrm{A}=\mathbb{C}$. The map $\mathfrak{a}^{\prime}: \mathbf{U} \rightarrow \mathbf{U}_{\circ}$ in section 1.6 factors to a Poisson algebra anti-automorphism

$$
\mathfrak{a}^{\prime}: \mathcal{U} \rightarrow \mathcal{U}
$$

By 2.2.3(c) there is an algebra isomorphism

$$
\mathfrak{m}: \mathcal{O}\left(D^{*}\right) \rightarrow \mathcal{D}_{\triangleright}, f \otimes f^{\prime} \mapsto \ell_{\mathfrak{z} F}(f) \partial_{\triangleright} \mathfrak{a}_{\mathfrak{z} U}^{\prime}\left(f^{\prime}\right) .
$$

Setting $\hbar=l\left(q^{l}-q^{-l}\right)$ in A.10.2 we get a Poisson bracket on $\mathcal{D}_{\triangleright}$. We must prove that $\mathfrak{m}$ is a Poisson algebra homomorphism. The maps

$$
\ell \circ \mathfrak{z}_{F}: \mathcal{O}(G) \rightarrow \mathcal{D}_{\triangleright}, \quad \partial_{\triangleright} \circ \mathfrak{a}^{\prime} \circ \mathfrak{z}_{U}: \mathcal{O}\left(G^{*}\right) \rightarrow \mathcal{D}_{\triangleright}
$$

are Poisson algebra homomorphisms by 2.2.3(c). In other words, we have

$$
\mathfrak{m}\left(\left\{f^{\prime}, f\right\}\right)=\left\{\mathfrak{m}\left(f^{\prime}\right), \mathfrak{m}(f)\right\}, \quad \forall f, f^{\prime} \in \mathcal{O}(G) \text { or } \mathcal{O}\left(G^{*}\right) .
$$

Therefore we must prove that this equality holds again if $f \in \mathcal{O}(G), f^{\prime} \in \mathcal{O}\left(G^{*}\right)$.

To simplify, from now on we will omit the maps $\partial_{\triangleright}, \ell$. We have $\mathbb{C}=\mathcal{A} /(q-\tau)$ where $\mathcal{A}=\mathbb{C}\left[q^{ \pm 1}\right]$. By (2.6.7) there is a $\mathbb{C}$-linear map $\nu: \mathcal{U} \rightarrow \dot{\mathbf{U}}$ such that the following holds in $\mathcal{D}_{\triangleright}$ :

$$
\{u, f\}=u_{2}\left(\nu\left(u_{1}\right) \triangleright f\right), \quad \forall f \in \mathcal{F} .
$$

From (A.8.2) we get

$$
\phi \nu\left(x_{i} z_{i}\right)=\bar{e}_{i}, \quad \phi \nu\left(y_{i} z_{i}\right)=-\bar{f}_{i}, \quad \phi \nu\left(z_{\lambda}\right)=\bar{h}_{\lambda} / 2 .
$$

Therefore, if $f \in \mathcal{O}(G)$ and $f^{\prime} \in \mathcal{O}\left(G^{*}\right)$, then we have

$$
\begin{aligned}
& \left\{x_{i} z_{i}, \mathfrak{z}_{F}(f)\right\} z_{i}^{-1}=\nu\left(x_{i} z_{i}\right) \triangleright \mathfrak{z}_{F}(f)=\mathfrak{z}_{F}\left(\bar{e}_{i} \triangleright f\right), \\
& \left\{y_{i} z_{i}, \mathfrak{z}_{F}(f)\right\} z_{i}^{-1}=\nu\left(y_{i} z_{i}\right) \triangleright \mathfrak{z}_{F}(f)=-\mathfrak{z}_{F}\left(\bar{f}_{i} \triangleright f\right), \\
& 2\left\{z_{\lambda}, \mathfrak{z}_{F}(f)\right\} z_{\lambda}^{-1}=2 \nu\left(z_{\lambda}\right) \triangleright \mathfrak{z}_{F}(f)=\mathfrak{z}_{F}\left(\bar{h}_{\lambda} \triangleright f\right),
\end{aligned}
$$

We have also

$$
\mathfrak{a}^{\prime}\left(x_{i}\right)=-y_{i}, \quad \mathfrak{a}^{\prime}\left(y_{i}\right)=-x_{i}, \quad \mathfrak{a}^{\prime}\left(z_{\lambda}\right)=z_{\lambda} .
$$

Thus we obtain the following formulas:

$$
\begin{aligned}
& \left\{\mathfrak{a}^{\prime}\left(x_{i} z_{i}\right), \mathfrak{z}_{F}(f)\right\}=\mathfrak{a}^{\prime}\left(z_{i}\right) \mathfrak{z}_{F}\left(\bar{f}_{i} \triangleright f\right), \\
& \left\{\mathfrak{a}^{\prime}\left(y_{i} z_{i}\right), \mathfrak{z}_{F}(f)\right\}=-\mathfrak{a}^{\prime}\left(z_{i}\right) \mathfrak{z}_{F}\left(\bar{e}_{i} \triangleright f\right), \\
& 2\left\{\mathfrak{a}^{\prime}\left(z_{\lambda}\right), \mathfrak{z}_{F}(f)\right\}=\mathfrak{a}^{\prime}\left(z_{\lambda}\right) \mathfrak{z}_{F}\left(\bar{h}_{\lambda} \triangleright f\right) .
\end{aligned}
$$

For each $x \in \mathfrak{g}$ we consider the 1-form $x^{r}$ on $G^{*}$ introduced in the proof of A.8.6(b). Recall that $x^{r}=\iota\left(f_{2}\right) \mathrm{d} f_{1}$ if $x=f^{\sharp}$. See section 2.1 for the notation. Let $\bar{x}_{i}, \bar{y}_{i}, \bar{h}_{\lambda} \in \mathcal{O}\left(G^{*}\right)$ be as in the proof of 2.2.3(c). We have

$$
\mathrm{d}_{e}\left(\bar{x}_{i} \bar{z}_{i}\right)=\left(\bar{f}_{i}\right)^{\sharp}, \quad \mathrm{d}_{e}\left(\bar{y}_{i} \bar{z}_{i}\right)=-\left(\bar{e}_{i}\right)^{\sharp}, \quad \mathrm{d}_{e}\left(\bar{z}_{\lambda}\right)=\left(\bar{h}_{\lambda}\right)^{\sharp} / 2 .
$$


Thus we have also

$$
\mathrm{d}\left(\bar{x}_{i} \bar{z}_{i}\right)=\bar{z}_{i}\left(\bar{f}_{i}\right)^{r}, \mathrm{~d}\left(\bar{y}_{i} \bar{z}_{i}\right)=-\bar{z}_{i}\left(\bar{e}_{i}\right)^{r}, 2 \mathrm{~d} \bar{z}_{\lambda}=\bar{z}_{\lambda}\left(\bar{h}_{\lambda}\right)^{r} .
$$

Given $g^{*} \in G^{*}$, let $y \in \mathfrak{g}$ be such that $\mathrm{d}_{g^{*}} f^{\prime}=y^{r}\left(g^{*}\right)$. By A.8.6(b) the Poisson bracket on $\mathcal{O}\left(D^{*}\right)$ is such that

$$
\left\{f^{\prime}, f\right\}\left(g, g^{*}\right)=(y \triangleright f)(g) .
$$

Thus (A.8.9) yields

$$
\begin{aligned}
& \left\{\bar{x}_{i} \bar{z}_{i}, f\right\}=\bar{z}_{i}\left(\bar{f}_{i} \triangleright f\right), \\
& \left\{\bar{y}_{i} \bar{z}_{i}, f\right\}=-\bar{z}_{i}\left(\bar{e}_{i} \triangleright f\right), \\
& 2\left\{\bar{z}_{\lambda}, f\right\}=\bar{z}_{\lambda}\left(\bar{h}_{\lambda} \triangleright f\right) .
\end{aligned}
$$

Using this and (A.8.8) we get

$$
\mathfrak{m}\left(\left\{f^{\prime}, f\right\}\right)=\left\{\mathfrak{m}\left(f^{\prime}\right), \mathfrak{m}(f)\right\}, \quad \forall f \in \mathcal{O}(G), f^{\prime}=\bar{x}_{i} \bar{z}_{i}, \bar{y}_{i} \bar{z}_{i}, \bar{z}_{\lambda} .
$$

By the Leibniz rule this identity holds again for each $f \in \mathcal{O}\left(D^{*}\right)$ and each $f^{\prime}=$ $\bar{x}_{i} \bar{z}_{i}, \bar{y}_{i} \bar{z}_{i}, \bar{z}_{\lambda}$.

Now, the set of functions $f^{\prime} \in \mathcal{O}\left(G^{*}\right)$ such that $\mathfrak{m}\left(\left\{f^{\prime}, f\right\}\right)=\left\{\mathfrak{m}\left(f^{\prime}\right), \mathfrak{m}(f)\right\}$ for each $f \in \mathcal{O}\left(D^{*}\right)$ is a Poisson subalgebra. Thus we are done, because $\left\{\bar{x}_{i} \bar{z}_{i}, \bar{y}_{i} \bar{z}_{i}, \bar{z}_{\lambda}\right\}$ is a set of generators of the Poisson algebra $\mathcal{O}\left(G^{*}\right)$.

$(d)$ Set $\mathbf{A}=\mathbb{C}$. The fact that $\mathbf{D}_{\triangleright}$ is a Poisson order is routine using A.10.2(b). Let us concentrate on the other claim. Set $\mathbf{f}=\mathbf{F} / \mathcal{F}^{\varepsilon} \mathbf{F}$. It is an $\mathbf{u}^{e}$-algebra, because

$$
u \triangleright f=f \triangleleft u=\varepsilon(u) f, \quad \forall u \in \mathbf{u}, f \in \mathcal{F} .
$$

We must prove that the basic representation of $\mathbf{d}_{\triangleright}=\mathbf{f} \sharp \mathbf{u}$ on $\mathbf{f}$ is a simple module. Since we have

$$
\operatorname{dim}\left(\mathbf{d}_{\triangleright}\right)=\operatorname{dim}(\operatorname{End}(\mathbf{f}))=l^{2 n^{2}}
$$

it is enough to check that this representation is faithful. By 1.4.3(c) we are reduced to prove that the natural pairing $\mathbf{f} \times \mathbf{u} \rightarrow \mathrm{A}$ is nondegenerate. This is well known.

A.9. Good filtrations. Let $(\mathcal{A}, \mathrm{A}, \mathrm{k})$ be a modular triple. In this section we will assume that $\mathcal{A}, \mathrm{A}$ are local rings with residue field k. More precisely, $\mathcal{A}$ will be the localization of $\mathbb{Z}\left[q^{ \pm 1}\right]$ at the field k. For $\lambda \in X$ let $\mathbb{H}_{\mathrm{A}}^{0}(\lambda)$ be the $\dot{\mathbb{U}}_{\mathrm{A}^{-}}$ module induced from the character $\tau^{\lambda}$ of the subalgebra generated by $\dot{\mathbb{U}}_{0, \mathrm{~A}}, \dot{\mathbb{U}}_{-, \mathrm{A}}$. A module $V \in \boldsymbol{\mathcal { M }} \boldsymbol{o} \boldsymbol{d}^{\mathrm{lf}}\left(\dot{\mathbb{U}}_{\mathrm{A}}\right)$ has a good filtration if there is a positive filtration of $V$ by $\dot{\mathbb{U}}_{\mathrm{A}}$-submodules whose layers belong to $\left\{\mathbb{H}_{\mathrm{A}}^{0}(\lambda) ; \lambda \in X_{+}\right\}$.

Let $\pi \subset X$ be a saturated $\Sigma_{n}$-invariant subset, i.e., we have

$$
\lambda \in \pi \cap X_{+}, \lambda^{\prime}<\lambda, \quad \lambda^{\prime} \in X_{+} \Rightarrow \lambda^{\prime} \in \pi .
$$

Consider the left exact functor

$$
O_{\pi}: \boldsymbol{M}_{\boldsymbol{o d}} \boldsymbol{d}^{\mathrm{lf}}\left(\dot{\mathbb{U}}_{\mathrm{A}}\right) \rightarrow \boldsymbol{M}_{\boldsymbol{o}} \boldsymbol{d}^{\mathrm{lf}}\left(\dot{\mathbb{U}}_{\mathrm{A}}\right)
$$

such that $O_{\pi}(V) \subset V$ is the largest $\dot{\mathbb{U}}_{\mathrm{A}}$-submodule whose weights belong to $\pi$. For each integer $a>0$ we put

$$
\pi_{a}=\left\{\lambda \in X ; \sum_{i} \lambda_{i}=a, \lambda_{i} \geqslant 0\right\}
$$


For each integer $r>0$ we put also

$$
X_{r}=\bigcup_{r \geqslant a \geqslant b \geqslant 0}\left(\pi_{a}-b \omega_{n}\right) \subset X, \quad X_{r,+}=X_{r} \cap X_{+} .
$$

We will abbreviate $O_{r}(V)=O_{X_{r}}(V)$. If $V=\mathbb{F}_{\mathrm{A}}$, the A-module $O_{r}\left(\mathbb{F}_{\mathrm{A}}\right)$ refers to the natural left $\dot{U}_{\mathrm{A}}$-action on $\mathbb{F}_{\mathrm{A}}$. The right action would give the same filtration.

A.9.1. Proposition. (a) Let $V \in \mathcal{M} \boldsymbol{d}^{l f}\left(\dot{U}_{\mathcal{A}}\right)$. Assume that $V$ has a good filtration. Then $(V \otimes \mathrm{A})^{+} \otimes \mathrm{k}=(V \otimes \mathrm{k})^{+}$and $(V \otimes \mathrm{A})^{\pi} \otimes \mathrm{k}=(V \otimes \mathrm{k})^{\pi}$.

(b) The adjoint representations on $\mathbb{F}_{\mathcal{A}}, \mathbb{U}_{\mathcal{A}}^{\prime}, \mathbb{D}_{\mathcal{A}}, \mathbb{D}_{\mathcal{A}}^{\prime}$ and $\mathbb{E}_{\mathcal{A}}$ have good filtrations. So the natural left action on $\mathbb{F}_{\mathcal{A}}^{\pi}, \mathbb{E}_{\mathcal{A}}^{\pi}$ does as well.

Proof. (a) Fix a good filtration $\left(F_{r} V\right)$ of $V$. Set $\operatorname{gr}_{r}(V)=F_{r} V / F_{r-1} V$, a free $\mathcal{A}$-module of finite rank. Hence $F_{r} V$ is also a free $\mathcal{A}$-module of finite rank and $\left(F_{r} V\right) \otimes \mathrm{A}$ has a good filtration. By [APW, sec. 3.4] there is a spectral sequence

$$
E_{2}^{i,-j}=\operatorname{Tor}_{j}^{\mathrm{A}}\left(H^{i}\left(\dot{\mathbb{U}}_{\mathrm{A}},\left(F_{r} V\right) \otimes \mathrm{A}\right), \mathrm{k}\right) \Rightarrow H^{i-j}\left(\dot{\mathbb{U}}_{\mathrm{k}},\left(F_{r} V\right) \otimes \mathrm{k}\right) .
$$

Further, we have

$$
H^{>0}\left(\dot{\mathbb{U}}_{\mathrm{A}},\left(F_{r} V\right) \otimes \mathrm{A}\right)=0 .
$$

Therefore, the spectral sequence above implies that the canonical map

$$
H^{0}\left(\dot{\mathbb{U}}_{\mathrm{A}},\left(F_{r} V\right) \otimes \mathrm{A}\right) \otimes \mathrm{k} \rightarrow H^{0}\left(\dot{\mathbb{U}}_{\mathrm{k}},\left(F_{r} V\right) \otimes \mathrm{k}\right)
$$

is invertible. This implies the first assertion.

Now we concentrate on the second one. For each integer $m$ the character $[m]$ in (A.6.2) restricts to a character

$$
[m]: \mathbb{U}_{\tilde{\pi}, \mathcal{A}} \rightarrow \mathcal{A} .
$$

Tensoring $V$ with $[m]$ yields a new object $V[m] \in \boldsymbol{M} \boldsymbol{M d}\left(\dot{\mathbb{U}}_{\tilde{\pi}, \mathcal{A}}\right)$; compare A.6.3. Since the $\dot{U}_{\mathcal{A}}$-action on $V$ is locally finite we have an isomorphism

$$
(V \otimes \mathrm{A})^{\pi} \rightarrow \bigoplus_{m \geqslant 0} H^{0}\left(\dot{\mathbb{U}}_{\tilde{\pi}, \mathrm{A}}, V[m] \otimes \mathrm{A}\right) .
$$

By Frobenius reciprocity and the tensor identity we have

$$
H^{0}\left(\dot{\mathbb{U}}_{\tilde{\pi}, \mathrm{A}}, V[m] \otimes \mathrm{A}\right)=H^{0}\left(\dot{\mathbb{U}}_{\mathrm{A}}, V \otimes \mathbb{H}_{\mathcal{A}}^{0}\left(m \omega_{1}\right) \otimes \mathrm{A}\right) .
$$

See APW, sec. 2.12, 2.16]. Tensor products of finite dimensional modules with good filtrations have again a good filtration by $\left[\mathrm{P}\right.$, thm. 3.3]. Thus $V \otimes \mathbb{H}_{\mathcal{A}}^{0}\left(m \omega_{1}\right)$ has again a good filtration. Thus the second claim follows from the first one.

(b) By A.9.3 below the $\dot{\mathbb{U}}_{\mathcal{A}}^{e}$-module $\mathbb{F}_{\mathcal{A}}$ has a good filtration. So the adjoint $\dot{\mathbb{U}}_{\mathcal{A}}$-modules $\mathbb{F}_{\mathcal{A}}, \mathbb{U}_{\mathcal{A}}^{\prime}, \mathbb{D}_{\mathcal{A}}, \mathbb{D}_{\mathcal{A}}^{\prime}$ and $\mathbb{E}_{\mathcal{A}}$ have also good filtrations by [P, thm. 3.3].

Now we prove that the left $\dot{\mathbb{U}}_{\mathcal{A}}$-action on $\mathbb{F}_{\mathcal{A}}^{\pi}$ has a good filtration. The $\dot{\mathbb{U}}_{\mathcal{A}^{-}}$ module $\mathbb{F}_{\mathcal{A}} \otimes \mathbb{H}_{\mathcal{A}}^{0}\left(m \omega_{1}\right)$ such that $\dot{U}_{\mathcal{A}}$ acts on $\mathbb{F}_{\mathcal{A}}$ and $\dot{\mathbb{U}}_{\mathcal{A}}^{\circ}$ on the tensor product has a good filtration by $\left[\mathrm{P}\right.$, thm. 3.3]. Thus, so does the $\dot{\mathbb{U}}_{\mathcal{A}}$-module $H^{0}\left(\dot{\mathbb{U}}_{\mathcal{A}}^{\circ}, \mathbb{F}_{\mathcal{A}} \otimes\right.$ $\left.\mathbb{H}_{\mathcal{A}}^{0}\left(m \omega_{1}\right)\right)$ by A.9.2 below. Finally, we have

$$
\mathbb{F}_{\mathcal{A}}^{\pi}=\bigoplus_{m \geqslant 0} H^{0}\left(\dot{U}_{\mathrm{A}}^{\circ}, \mathbb{F}_{\mathcal{A}} \otimes \mathbb{H}_{\mathcal{A}}^{0}\left(m \omega_{1}\right)\right) .
$$

The proof for $\mathbb{E}_{\mathcal{A}}^{\pi}$ is the same.

A.9.2. Lemma. For any locally finite $\dot{U}_{\mathcal{A}}^{e}$-module $V$ with a good filtration the $\dot{\mathbb{U}}_{\mathcal{A}}$-module $H^{0}\left(\dot{\mathbb{U}}_{\mathcal{A}}^{\circ}, V\right)$ again has a good filtration. 
Proof. Fix a positive filtration $\left(F_{r} V\right)$ of $V$ by $\dot{U}_{\mathcal{A}}^{e}$-submodules whose layers are of the form $\mathbb{H}_{\mathcal{A}}^{0}(\lambda) \otimes \mathbb{H}_{\mathcal{A}}^{0}(\mu)$ with $\lambda, \mu \in X_{+}$. The $\dot{\mathbb{U}}_{\mathcal{A}}^{\circ}$-module $F_{r} V$ has a good filtration for each $r$. Thus $H^{>0}\left(\dot{U}_{\mathcal{A}}^{\circ}, F_{r} V\right)=0$. Hence there is a short exact sequence

$$
0 \rightarrow H^{0}\left(\dot{\cup}_{\mathcal{A}}^{\circ}, F_{r} V\right) \rightarrow H^{0}\left(\dot{\mathbb{U}}_{\mathcal{A}}^{\circ}, F_{r+1} V\right) \rightarrow \mathbb{H}_{\mathcal{A}}^{0}(\lambda) \otimes H\left(\dot{U}_{\mathcal{A}}^{\circ}, \mathbb{H}_{\mathcal{A}}^{0}(\mu)\right) \rightarrow 0 .
$$

So the A-modules $H^{0}\left(\dot{U}_{\mathcal{A}}^{\circ}, F_{r} V\right), r \geqslant 0$, yield a good filtration of $H^{0}\left(\dot{U}_{\mathcal{A}}^{\circ}, V\right)$.

The following is standard; compare [J1, sec. A.15].

A.9.3. Lemma. For each integer $r$ the $\dot{U}_{\mathcal{A}}^{e}$-module $O_{r}\left(\mathbb{F}_{\mathcal{A}}\right)$ is a free $\mathcal{A}$-module of finite rank. It has a good filtration.

A.10. Maximal orders, Poisson orders and Azumaya algebras. All rings or algebras are assumed to be unital.

A central simple algebra (over $\mathrm{k}$ ) is a simple algebra $\mathbf{A}$ over a field $\mathrm{k}$ which is finite dimensional over $\mathrm{k}$ and such that $\mathrm{k}=Z(\mathbf{A})$, the center of $\mathbf{A}$.

An element of a ring is regular if it is not a zero divisor. A ring $\mathbf{B}$ is a quotient ring if every regular element is a unit; see [MR, sec. 3.1.1]. See also [MR, sec. 2.1.14] for the definition of the quotient ring of a ring $\mathbf{A}$. Let $\operatorname{Frac}(\mathbf{A})$ denote the quotient ring of $\mathbf{A}$ whenever it is defined.

A subring $\mathbf{A}$ of a quotient ring $\mathbf{B}$ is an order (in $\mathbf{B}$ ) if every element of $\mathbf{B}$ has the form $a_{1} b_{1}^{-1}=b_{2}^{-1} a_{2}$ for some $a_{1}, a_{2}, b_{1}, b_{2} \in \mathbf{A}$; see [MR, sec. 3.1.2]. Then $\mathbf{B}=\operatorname{Frac}(\mathbf{A})$. Further, a ring is an order in its quotient ring whenever the latter is defined; see [MR, sec. 3.1.4]. An order $\mathbf{A}$ in a quotient ring $\mathbf{B}$ is maximal if for any order $\mathbf{A}^{\prime} \subset \mathbf{B}$ and any $a_{1}, a_{2} \in \mathbf{A}$ such that $\mathbf{A} \subset a_{1} \mathbf{A}^{\prime} a_{2}$ we have $\mathbf{A}=\mathbf{A}^{\prime}$; see [MR, sec. 5.1.1, 3.1.9]. By a maximal order we will always mean a ring with a quotient ring which is a maximal order in its quotient ring.

For the definition of a PI-ring see [BG3, sec. I.13.1]. A ring which is a module of finite type over a commutative subring is a PI-ring; see [MR, cor. 13.1.13(iii)].

An order in a central simple algebra is a prime ring. Conversely, let $\mathbf{A}$ be a prime PI-ring. Then $Z(\mathbf{A})$ is a CID and $\mathbf{A}$ is an order in its quotient ring. The latter is equal to $\operatorname{Frac}(Z(\mathbf{A})) \otimes_{Z(\mathbf{A})} \mathbf{A}$, a central simple algebra over $\operatorname{Frac}(Z(\mathbf{A}))$. This is Posner's theorem; see [MR, thm. 13.6.5] for details.

For future use, let us quote the following.

A.10.1. Proposition. (a) Let $\mathbf{A}$ be a prime ring which is a module of finite type over $Z(\mathbf{A})$. Assume that $\mathbf{A}$ is a maximal order and that $\mathbf{B} \subset \operatorname{Frac}(\mathbf{A})$ is a subring containing $\mathbf{A}$. If $\mathbf{B}$ is a $Z(\mathbf{A})$-module of finite type, then we have $\mathbf{A}=\mathbf{B}$.

(b) Let $\mathbf{A}$ be a NID and let $\phi \in A u t(\mathbf{A})$. The twisted polynomial ring $\mathbf{A}[x ; \phi]$ is a NID. If $\phi^{l}=1$ and $\mathbf{A}$ is a maximal order, then $\mathbf{A}[x ; \phi]$ is a maximal order.

Proof. (a) The ring $\mathbf{B}$ is an order in $\operatorname{Frac}(\mathbf{A})$ by $[\mathrm{MR}$, cor. 3.1.6(i)] and $\operatorname{Frac}(\mathbf{A})=$ $\operatorname{Frac}(Z(\mathbf{A})) \otimes_{Z(\mathbf{A})} \mathbf{A}$. Then, since $\mathbf{B}$ is $Z(\mathbf{A})$-module of finite type there is an element $0 \neq z \in Z(\mathbf{A})$ such that $\mathbf{B} \subset z^{-1} \mathbf{A}$. Thus $\mathbf{B}=\mathbf{A}$, because $\mathbf{A}$ is maximal in its equivalence class.

(b) The first assertion is proved in [MR, thm. 1.2.9]. Let us concentrate on the second one. Recall that $\mathbf{A}[x ; \phi]$ is the algebra generated by $\mathbf{A}$ and $x$ with the additional relations

$$
\left(a \otimes x^{i}\right)\left(b \otimes x^{j}\right)=a \phi^{i}(b) \otimes x^{i+j} .
$$

By [DP, sec. 6.3] it is an order in its quotient ring. We must check that it is integrally closed. Fix an element $0 \neq z$ in $Z(\mathbf{A}[x ; \phi])$ and let $\mathbf{A}^{\prime}$ be an algebra such 
that

$$
\mathbf{A}[x ; \phi] \subset \mathbf{A}^{\prime} \subset z^{-1} \mathbf{A}[x ; \phi] .
$$

We must check that $\mathbf{A}[x ; \phi]=\mathbf{A}^{\prime}$. Let $l$ be the order of $\phi$. We will write $y \equiv y^{\prime}$ if $y, y^{\prime} \in \mathbf{A}[x ; \phi]$ coincide up to terms of lower degree in $x$. Fix $b \neq 0$ such that $z \equiv b x^{r}$. Since $z$ is a central element, we get $a b x^{r}=b x^{r} a$ for all $a \in \mathbf{A}$. Thus $a b=b \phi^{r}(a)$ for all $a$. So $b^{l} \in Z(\mathbf{A})$. On the other hand, for each $k \geqslant 1$ an obvious induction yields

$$
z^{k} \equiv z^{k-1} b x^{r} \equiv b z^{k-1} x^{r} \equiv b\left(b^{k-1} x^{(k-1) r}\right) x^{r} \equiv b^{k} x^{k r} .
$$

In particular, $z^{l} \equiv b^{l} x^{l r}$. So, up to changing $z$ with $z^{l}$, we can assume that $z \equiv b x^{r}$ and that $b$ belongs to $Z(\mathbf{A})$.

Fix $y$ in $\mathbf{A}^{\prime}$ and $0 \neq a$ in $\mathbf{A}$ such that $z y \equiv a x^{s}$. For $m \geqslant s$ we have $z y x^{m-s} \equiv a x^{m}$. Put $m=l(s+1)$. Then $z y x^{m-s} \equiv a x^{l(s+1)}$. So for each $k \geqslant 1$ we get $\left(z y x^{m-s}\right)^{k} \equiv a^{k} x^{k m}$. Furthermore, $\left(z y x^{m-s}\right)^{k}=z^{k-1} z\left(y x^{m-s}\right)^{k}$, with $z\left(y x^{m-s}\right)^{k} \in \mathbf{A}[x ; \phi]$ and $z^{k-1} \equiv b^{k-1} x^{(k-1) r}$. Thus $a^{k} \in b^{k-1} \mathbf{A}$. Recall that $Z(\mathbf{A})$ is a CID and that $0 \neq b$ belongs to $Z(\mathbf{A})$. Thus $(a / b)^{k} \in b^{-1} \mathbf{A}$ for each $k$, where both terms are viewed inside $\operatorname{Frac}(Z(\mathbf{A})) \otimes_{Z(\mathbf{A})} \mathbf{A}$. Thus $c=a / b \in \mathbf{A}$, because $\mathbf{A}$ is integrally closed.

Recall that $z y \equiv a x^{s}$ and $z \equiv b x^{r}$. We claim that $s \geqslant r$. Indeed, for each $k \geqslant 1$ there is an element $a^{\prime} \in \mathbf{A}$ such that

$$
a^{\prime} x^{k s} \equiv(z y)^{k} \equiv b^{k-1} x^{(k-1) r} z y^{k}
$$

(note that $y^{k} \in \mathbf{A}^{\prime}$ ). Hence $k s \geqslant(k-1) r$ for each $k \geqslant 1$.

We can now prove that $y \in \mathbf{A}[x ; \phi]$. Assume that $y \notin \mathbf{A}[x ; \phi]$. Choose $y$ such that $s$ is minimal. Put $y^{\prime}=y-c x^{s-r}$. Note that $c x^{s-r} \in \mathbf{A}[x ; \phi]$, because $s \geqslant r$ and $c \in \mathbf{A}$. The element $c z x^{s-r}$ has the same leading term as $z y$. Since $c z x^{s-r}=z c x^{s-r}$, we get that $y^{\prime} \in \mathbf{A}^{\prime}, z y^{\prime} \in \mathbf{A}[x ; \phi]$ and $z y^{\prime}$ has a degree $<s$. By minimality of $s$ we have that $y^{\prime} \in \mathbf{A}[x ; \phi]$. Hence $y=y^{\prime}+c x^{s-r} \in \mathbf{A}[x ; \phi]$, which gives a contradiction.

Let $\mathbf{A}$ be an algebra over a commutative ring A. A trace is an A-linear endomorphism tr $: \mathbf{A} \rightarrow \mathbf{A}$ such that

$$
\operatorname{tr}(a b)=\operatorname{tr}(b a), \quad \operatorname{tr}(a) b=b \operatorname{tr}(a), \quad \operatorname{tr}(\operatorname{tr}(a) b)=\operatorname{tr}(a) \operatorname{tr}(b), \quad \forall a, b .
$$

Let $\mathrm{B}=\operatorname{tr}(\mathbf{A})$. Note that $\mathrm{B} \subset Z(\mathbf{A})$, a subalgebra, that $\operatorname{tr}$ is $\mathrm{B}$-linear and that $\mathbf{A}=\beta \oplus \operatorname{Ker}(\operatorname{tr})$ as B-modules.

Let $\mathbf{A}$ be a prime ring which is a module of finite type over a subring of $Z(\mathbf{A})$. The simple algebra $\operatorname{Frac}(\mathbf{A})$ is equipped with the reduced trace. Let $B=\operatorname{tr}(\mathbf{A})$. If $\mathbb{C} \subset Z(\mathbf{A})$ and $Z(\mathbf{A})$ is an integrally closed domain, then $\mathrm{B} \subset Z(\mathbf{A})$. If $\mathbf{A}$ is a maximal order, then $Z(\mathbf{A})$ is an integrally closed domain and $\mathrm{B}=Z(\mathbf{A})$. Thus $Z(\mathbf{A})$ is a direct summand of $\mathbf{A}$ as a $Z(\mathbf{A})$-module.

For the definition of an Azumaya algebra (over its center) see [BG3, sec. III.1.3] for instance. More generally, let $X$ be a Noetherian A-scheme. An Azumaya algebra over $X$ is a sheaf of $\mathcal{O}_{X}$-algebras $\mathcal{E} \in \operatorname{Coh}\left(\mathcal{O}_{X}\right)$ such that $\mathcal{E}(U)$ is an Azumaya algebra over $\mathcal{O}(U)$ for each open subset $U \subset X$.

Let $\mathbf{A}$ be an affine algebra over an algebraically closed field $\mathrm{k}$. Assume that $\mathbf{A}$ is prime and is a module of finite type over a subalgebra of $Z(\mathbf{A})$. Then $Z(\mathbf{A})$ is a CID and there is a multiplicative subset $S \subset Z(\mathbf{A})$ such that $S^{-1} \mathbf{A}$ is an Azumaya algebra over $S^{-1} Z(\mathbf{A})$. See [BG3, sec. III.1.7]. 
Let $\mathbf{A}$ be an affine algebra over a Noetherian commutative ring A. Assume that $\mathbf{A}$ is a module of finite type over an A-subalgebra $\mathrm{B} \subset Z(\mathbf{A})$. Note that the Aalgebra B is finitely generated by the Artin-Tate lemma, see [BG3, sec. I.13.4] and the references therein. Following [BG2] we say that $\mathbf{A}$ is a Poisson order over B, or over $\operatorname{Spec}(\mathrm{B})$, if there is an A-linear map

$$
\theta: \mathrm{B} \rightarrow \operatorname{Der}_{\mathrm{A}}(\mathbf{A}), b \mapsto \theta_{b}
$$

and an A-linear Poisson bracket on B such that $\theta_{b}\left(b^{\prime}\right)=\left\{b, b^{\prime}\right\}$ for all $b, b^{\prime} \in \mathrm{B}$.

Now, let $X$ be an A-scheme of finite type. A Poisson order over $X$ is a coherent sheaf of $\mathcal{O}_{X}$-algebras $\mathcal{E}$ such that $\mathcal{E}(U)$ is a Poisson order over $U$ for each open subset $U \subset X$.

Next, let $f: X \rightarrow Y$ be a Poisson A-scheme homomorphism, i.e., a A-scheme homomorphism such that the canonical map

$$
f^{*}: f^{-1} \mathcal{O}_{Y} \rightarrow \mathcal{O}_{X}
$$

is compatible with the Poisson brackets. For any Poisson order $\mathcal{F}$ over $Y$ the sheaf $f^{*} \mathcal{F}$ has a natural structure of a Poisson order over $X$. Given a Poisson order $\mathcal{E}$ over $X$, a morphism of Poisson orders $\mathcal{F} \rightarrow \mathcal{E}$ is a morphism of $\mathcal{O}_{X}$-algebras $f^{*} \mathcal{F} \rightarrow \mathcal{E}$ which is compatible with the A-linear maps

$$
\mathcal{O}(U) \rightarrow \operatorname{Der}_{\mathrm{A}}(\mathcal{E}(U)), \quad \mathcal{O}(U) \rightarrow \operatorname{Der}_{\mathrm{A}}\left(\left(f^{*} \mathcal{F}\right)(U)\right)
$$

We may also use a relative version of Poisson orders. Let $f: X \rightarrow Y$ be a morphism of A-schemes of finite type. To simplify we will assume that $Y$ is an affine Poisson scheme. Let $\mathcal{E}$ be a coherent sheaf of $\mathcal{O}_{X}$-algebras. The composition by $f$ yields an algebra homomorphism $\phi_{f}: \mathcal{O}(Y) \rightarrow \mathcal{E}$. We will say that $\mathcal{E}$ is a Poisson order over $f$ if there is an A-linear map $\theta: \mathcal{O}(Y) \rightarrow \operatorname{Der}_{\mathrm{A}}(\mathcal{E})$ such that

$$
\theta_{b}\left(\phi_{f}\left(b^{\prime}\right)\right)=\phi_{f}\left(\left\{b, b^{\prime}\right\}\right), \quad \forall b, b^{\prime} \in \mathcal{O}(Y) .
$$

Note that a Poisson order over $X$ is also a Poisson order over $f$.

Recall the Hayashi lemma.

A.10.2. Proposition. Fix an A-algebra $\mathbf{A}$ and an element $\hbar \in \mathrm{A}$ which is regular in $\mathbf{A}$. Let $\mathbf{A} \rightarrow \overline{\mathbf{A}}=\mathbf{A} / \hbar \mathbf{A}$ and $a \mapsto \bar{a}$ be the canonical map.

(a) Fix an element $a \in \mathbf{A}$ such that $\bar{a} \in Z(\overline{\mathbf{A}})$. For each $b \in \mathbf{A}$ there is a unique element $\langle a, b\rangle \in \mathbf{A}$ such that $\hbar\langle a, b\rangle=[a, b]$. The assignment $\theta_{a}: \bar{b} \mapsto \overline{\langle a, b\rangle}$ defines a derivation of $\overline{\mathbf{A}}$.

(b) The assignment $(\bar{a}, \bar{b}) \mapsto \theta_{a}(\bar{b})$ gives a Poisson bracket on $Z(\overline{\mathbf{A}})$. If $\overline{\mathbf{A}}$ is a $Z(\overline{\mathbf{A}})$-module of finite type, then it is a Poisson order over $Z(\overline{\mathbf{A}})$.

Note that the derivation $\theta_{a} \in \operatorname{Der}(\overline{\mathbf{A}})$ depends on $a$ but that its restriction to $Z(\overline{\mathbf{A}})$ depends only on $\bar{a}$. We will also need the following standard result.

A.10.3. Proposition. (a) Let $\mathbf{A}$ be a Poisson order over B. If $\mathrm{A}=\mathbb{C}$ and the points $x, y: \mathrm{B} \rightarrow \mathbb{C}$ belong to the same symplectic leaf, then the fibers of $\mathbf{A}$ at $x, y$ are isomorphic as $\mathbb{C}$-algebras.

(b) Let $\mathcal{E}$ be a Poisson order over a proper map $f: X \rightarrow Y$ with $Y$ affine. Then $H^{\bullet}(X, \mathcal{E})$ is a Poisson order over $Y$.

(c) Let $f: X \rightarrow Y$ be a Poisson scheme homomorphism. Given a morphism of Poisson orders $\mathcal{F} \rightarrow \mathcal{E}$, the image and the cokernel of the natural map $f^{*} \mathcal{F} \rightarrow \mathcal{E}$ are Poisson orders over $X$. 
Proof. (a) See [DP, sec. 11.8] and [BG2, thm. 4.2].

(b) First note that $H^{\bullet}(X, \mathcal{E})$ is a $\mathcal{O}(Y)$-module of finite type. Further, as $X$ is a Noetherian separated scheme $H^{\bullet}(X, \mathcal{E})$ is the cohomology of the Cech complex of an affine open cover $X=\bigcup_{i \in I} U_{i}$. The map $\theta$ factors to $\mathcal{O}(Y) \rightarrow \operatorname{Der}_{\mathrm{A}}\left(\mathcal{E}\left(U_{J}\right)\right)$ for each intersection $U_{J}=\bigcap_{i \in J} U_{i}$. As it commutes with coboundary maps of the Cech complex, the cohomology ring $H^{\bullet}(X, \mathcal{E})$ is a Poisson order over $Y$.

(c) Obvious.

\section{A.11. Proof of 2.3.}

Proof of 2.3.3. (a) Fix $V \in \mathcal{M}^{\text {od }}{ }^{\text {lf }}\left(\dot{\mathbb{U}}_{\mathrm{A}}\right)$. We must prove that $V^{+}$is A-flat. This is done as in APW, 3.5-3.6], using the standard resolution of $V$ constructed there.

The second assertion follows from the universal coefficient theorem. Since $a$ acts regularly on $V$ the module $V$ embeds into the module of quotients $V_{a}$. So $V^{+}=V \cap\left(V_{a}\right)^{+}$. Hence $a$ also acts regularly on the quotient $V / V^{+}$. Since A is an ID this yields $\operatorname{Tor}_{1}^{\mathrm{A}}\left(V / V^{+}, \mathrm{k}\right)=0$. Thus $V^{+} \otimes \mathrm{k} \subset(V \otimes \mathrm{k})^{+}$.

The last claim in $(a)$ is identical.

(b) Follows from A.9.1 and [P, thm. 3.3].

Proof of 2.3.4. (a) The maps $\ell \otimes \partial_{\triangleleft}$ and $\ell \otimes\left(\partial_{\triangleright} \circ \kappa\right)$ yield A-module isomorphisms $\mathbb{D}_{\mathrm{A}} \simeq \mathbb{F}_{\mathrm{A}} \otimes \mathbb{U}_{\mathrm{A}}^{\prime}$ and $\mathbb{D}_{\mathrm{A}}^{\prime} \simeq \mathbb{F}_{\mathrm{A}} \otimes \mathbb{F}_{\mathrm{A}}$. The map $\psi$ gives an $\alpha$-module isomorphism

$$
\mathbb{S}_{\mathrm{A}} \rightarrow \mathbb{F}_{\mathrm{A}} \otimes \mathbb{V}_{\mathrm{A}} \otimes \mathbb{F}_{\mathrm{A}} \otimes \mathbb{F}_{\mathrm{A}} \text {. }
$$

It factors to an A-module isomorphism $\mathbb{S}_{\mathrm{A}, i} \rightarrow \mathbb{F}_{\mathrm{A}, i} \otimes \mathbb{V}_{\mathrm{A}} \otimes \mathbb{F}_{\mathrm{A}} \otimes \mathbb{F}_{\mathrm{A}}$ for all $i$. Thus $\mathbb{S}_{\mathrm{A}}$ is $\mathrm{A}$-free, $\mathbb{S}_{\mathrm{A}, i}$ is A-flat and $\mathbb{S}_{\mathrm{A}} \subset \mathbb{S}_{\mathrm{A}, i}$ because $\mathbb{F}_{\mathrm{A}}$ is a domain.

We have $\mathbb{R}_{\mathrm{A}} \simeq \mathbb{F}_{\mathrm{A}} \otimes \mathbb{V}_{\mathrm{A}}$ and $\mathbb{R}_{\mathrm{A}, i} \simeq \mathbb{F}_{\mathrm{A}, i} \otimes \mathbb{V}_{\mathrm{A}}$. So $\mathbb{R}_{\mathrm{A}}$ is A-free, $\mathbb{R}_{\mathrm{A}, i}$ is Aflat, $\mathbb{R}_{\mathrm{A}} \subset \mathbb{R}_{\mathrm{A}, i}$ and $\mathbb{R}_{\mathrm{A}}^{\pi} \subset \mathbb{R}_{\mathrm{A}, i}^{\pi}$. The action of $u \in \dot{\mathbb{U}}_{\tilde{\pi}, \mathrm{A}}$ on $\mathbb{R}_{\mathrm{A}}$ takes $f \otimes v$ to $\left(f \triangleleft \iota\left(u_{2}\right)\right) \otimes\left(\operatorname{ad} u_{1}\right)(v)$. Thus it is locally finite. So $\mathbb{R}_{\mathrm{A}}^{\pi}, \mathbb{R}_{\mathrm{A}, i}^{\pi}$ are A-flat by 2.3.3(a).

Now we consider only the A-module $\mathbb{R}_{\mathrm{A}}$. The proofs for $\mathbb{S}_{\mathrm{A}}$ is identical.

(b) On localizing and taking $\dot{U}_{\pi, \mathrm{A}}$-invariants the natural map $\mathbb{D}_{\mathrm{A}} \rightarrow \mathbb{R}_{\mathrm{A}}$ factors to $\mathbb{D}_{\mathrm{A}, i}^{\pi} \rightarrow \mathbb{R}_{\mathrm{A}, i}^{\pi}$. Assume initially that $\mathrm{A}$ is a field. The argument in A.6.3 $(a)$ yields an exact functor

$$
\operatorname{Mod}^{\mathrm{lf}}\left(\mathbb{U}_{\pi, \mathrm{A}}\right) \rightarrow \operatorname{Mod}\left(\mathbb{F}_{\mathrm{A}, i}^{\pi}\right), \quad V \mapsto\left(\mathbb{F}_{\mathrm{A}, i} \otimes V\right)^{\pi} .
$$

Thus the map $\mathbb{D}_{\mathrm{A}, i}^{\pi} \rightarrow \mathbb{R}_{\mathrm{A}, i}^{\pi}$ is surjective. If $\alpha$ is no longer a field, it is enough to check that the $q$-analogue of Kempf's vanishing theorem holds again. In $[\mathrm{R}$ it is proved for fields. This is a consequence of [APW]. Indeed, let $\lambda \in X_{+}$. For any ring homomorphism $\mathrm{A} \rightarrow \mathrm{k}$ with $\mathrm{k}$ a field we have $\mathbb{H}_{\mathrm{A}}^{\bullet}(\lambda) \otimes \mathrm{k}=\mathbb{H}_{\mathrm{k}}^{\bullet}(\lambda)$ by base change; see [APW] sec. 3.3]. Here $\mathbb{H}_{\mathrm{A}}^{\bullet}(\lambda)$ is as in section A.9. Thus $\mathbb{H}_{\alpha}^{>0}(\lambda) \otimes \mathrm{k}=0$ for all $\mathrm{k}$. Thus $\mathbb{H}_{\mathrm{A}}^{>0}(\lambda)=0$. So it vanishes again for any A by base change again.

(c) The equality $\mathbb{R}_{\mathrm{A}} \otimes \mathrm{k}=\mathbb{R}_{\mathrm{k}}$ is obvious. Let us prove the corresponding assertion for $\mathbb{R}_{\mathrm{A}}^{\pi}$. The $\iota\left(\mathbb{U}_{\pi}\right) \otimes \mathbb{U}$-module isomorphism $\mathbb{R}_{+} \simeq \mathbb{F} \otimes^{\pi} \mathbb{F}$ given by 1.7.2(b) yields the $\iota\left(\dot{U}_{\pi, \mathrm{A}}\right) \otimes \dot{\mathbb{U}}_{\mathrm{A}}$-submodule $\mathbb{R}_{+, \mathrm{A}} \simeq \mathbb{F}_{\mathrm{A}} \otimes{ }^{\pi} \mathbb{F}_{\mathrm{A}}$ of $\mathbb{R}_{\mathrm{A}}$. Here $\dot{\mathbb{U}}_{\mathrm{A}}$ acts on $\mathbb{F}_{\mathrm{A}}$ from the left and $\iota\left(\dot{U}_{\pi, \mathrm{A}}\right)$ on both $\mathbb{F}_{\mathrm{A}},{ }^{\pi} \mathbb{F}_{\mathrm{A}}$ from the right. Furthermore, $\mathbb{R}_{\mathrm{A}}$ is the localization of $\mathbb{R}_{+, \mathrm{A}}$ at $c=1 \otimes c_{11}$. Since $c$ is $\dot{\mathbb{U}}_{\pi, \mathrm{A}}$-invariant and direct limits commute with tensor products and taking invariants, then 2.3.3(b) yields

$$
\mathbb{R}_{\mathrm{A}}^{\pi} \otimes \mathrm{k}=\left(\lim _{n} c^{-n} \mathbb{R}_{+, \mathrm{A}}\right)^{\pi} \otimes \mathrm{k}=\lim _{\lim _{n}} c^{-n}\left(\mathbb{R}_{+, \mathrm{A}}^{\pi} \otimes \mathrm{k}\right)=\lim _{\lim _{n}} c^{-n} \mathbb{R}_{+, \mathrm{k}}^{\pi}=\mathbb{R}_{\mathrm{k}}^{\pi}
$$


Proof of 2.3.6. (a) Taking tensor products is right exact.

(b) Identify $\mathbb{S}_{\mathrm{A}}$ with $\mathbb{R}_{\mathrm{A}} \otimes \mathbb{D}_{\mathrm{A}}^{\prime}$ via the map $\psi$. Let $\mathbb{S}_{+, \mathrm{A}}^{\pi}=\mathbb{R}_{+, \mathrm{A}}^{\pi} \otimes \mathbb{D}_{\mathrm{A}}^{\prime}$. Let $c \in \mathbb{R}_{+, \mathrm{A}}^{\pi}$ be as above. We abbreviatte $c=\psi(c \otimes 1)$, an element in $\mathbb{S}_{+, \mathrm{A}}^{\pi}$. Note that $\mathbb{S}_{\mathrm{A}}^{\pi}$ is the localization of the subalgebra $\mathbb{S}_{+, \mathrm{A}}^{\pi}$ at $c$.

We claim that the adjoint $\dot{U}_{\mathcal{A}}$-action on $\mathbb{S}_{+, \mathcal{A}}^{\pi}$ has a good filtration. It is enough to check that the left action also has a good filtration on $\mathbb{R}_{+, \mathcal{A}}^{\pi}$ by $[\mathrm{P}$, thm. 3.3] and A.9.1(b). The $\dot{U}_{\mathcal{A}}$-module $\mathbb{R}_{+, \mathcal{A}}^{\pi}$ is the tensor product of the left $\dot{U}_{\mathcal{A}}$-action on $\mathbb{F}_{\mathcal{A}}^{\pi}$ and the contragredient left $\dot{U}_{\mathcal{A}}$-action on ${ }^{\pi} \mathbb{F}_{\mathcal{A}}$. Thus the claim follows from $[\mathrm{P}$, thm. 3.3] again.

Now part $(b)$ follows from A.9.1( $a$ ) as in the proof of 2.3.4(c).

(c) Recall that $T_{\pi}$ is a closed subset of $\bar{S}_{\pi}=D \times \bar{R}_{\pi}$. Since $\tau=1$ in $\mathrm{k}_{c}$ we have

$$
\mathbf{S}_{\mathrm{k}_{c}}^{\pi}=\mathcal{O}\left(\bar{S}_{\pi, \mathrm{k}_{c}}\right), \quad \mathbf{T}_{\mathrm{k}_{c}}=\mathcal{O}\left(T_{\pi, \mathrm{k}_{c}}\right) .
$$

See 2.5.7 $(a)$ and 2.6.11(a) for details. Furthermore, part $(a)$ and 2.3.4(c) yield

$$
\mathbf{S}_{c}^{\pi} \otimes \mathrm{k}_{c}=\mathbf{S}_{\mathrm{k}_{c}}^{\pi}, \quad \mathbf{T}_{c} \otimes \mathrm{k}_{c}=\mathbf{T}_{\mathrm{k}_{c}} .
$$

Next, we have $\mathbf{T}_{c}=\mathbf{S}_{c}^{\pi} / \mathbf{J}_{c}$ and the left ideal $\mathbf{J}_{c} \subset \mathbf{S}_{c}^{\pi}$ is generated by the set

$$
\left\{\partial_{c} \kappa\left(c_{i j}\right)-\tau^{-2} \zeta^{2} \delta_{i j}\right\} \text {. }
$$

Under specialization to $\mathrm{k}_{c}$ this set yields a regular sequence in $\mathcal{O}\left(\bar{S}_{\pi, \mathrm{k}_{c}}\right)$, because $T_{\pi, \mathrm{k}_{c}} \subset \bar{S}_{\pi, \mathrm{k}_{c}}$ is a complete intersection of codimension $n^{2}$ by $4.1 .1(b)$. Recall that $\mathrm{A}_{c}$ is a DVR with residue field $\mathrm{k}_{c}$. To prove that the $\mathrm{A}_{c}$-modules $\mathbf{T}_{c}, \mathbf{T}_{c}^{+}$are flat it is enough to prove that $\mathbf{T}_{c}$ is torsion free. By 2.3.4(a) the $\mathrm{A}_{c}$-module $\mathbf{S}_{c}^{\pi}$ is flat. Therefore we are reduced to check that the elements in (A.11.1) satisfy the relations in A.11.2 below. Recall that the symbol $c_{i j}$ in (A.11.1) denotes an element in $\mathbb{F}_{+}^{\prime}$; see the definitions of $\partial_{c}$ and $\kappa$ in section 1.10 and Proposition 1.7.1 $(a)$. The defining relations of $\mathbb{F}_{+}^{\prime}$ in $1.7 .1(b)$ can be rewriten in the following way (left to the reader):

$$
\begin{aligned}
q^{\delta_{m i}+\delta_{m j}} c_{l m} c_{i j}-q^{\delta_{i l}+\delta_{j l}} c_{i j} c_{l m} & =\left(q-q^{-1}\right) q^{\delta_{i j}}\left(\delta_{i>l}-\delta_{j>m}\right) c_{l j} c_{i m} \\
& +\left(q-q^{-1}\right) q^{\delta_{i j}}\left(\sum_{j>p} \delta_{j l} c_{i p} c_{p m}-\sum_{m>p} \delta_{i m} c_{l p} c_{p j}\right) \\
& +\left(q-q^{-1}\right)^{2} \delta_{i j}\left(\delta_{i>l}-\delta_{j>m}\right) \sum_{j>p} c_{l p} c_{p m}, \quad \forall i, j, l, m .
\end{aligned}
$$

So the flatness of the $\mathrm{A}_{c}$-modules $\mathbf{T}_{c}, \mathbf{T}_{c}^{+}$is reduced to a routine computation.

Now, consider the commutative square

$$
\begin{array}{ccc}
\mathbf{S}_{c}^{\pi,+} \otimes \mathrm{k}_{c} & \rightarrow & \mathbf{T}_{c}^{+} \otimes \mathrm{k}_{c} \\
\downarrow & & \downarrow \\
\mathbf{S}_{\mathrm{k}_{c}}^{\pi,+} & \rightarrow & \mathbf{T}_{\mathrm{k}_{c}}^{+} .
\end{array}
$$

The lower map in the diagram is the restriction

$$
\mathcal{O}\left(\bar{S}_{\pi, \mathrm{k}_{c}}\right)^{+} \rightarrow \mathcal{O}\left(T_{\pi, \mathrm{k}_{c}}\right)^{+}
$$

It is surjective by A.11.3, because $p$ is large. The right map is injective because $\mathbf{T}_{c}$ is $\mathrm{A}_{c}$-flat; see 2.3.3(a). The left map is invertible by part $(b)$. Therefore the right map is invertible, i.e., we have $\mathbf{T}_{c}^{+} \otimes \mathrm{k}_{c}=\mathbf{T}_{\mathrm{k}_{c}}^{+}$.

A.11.2. Lemma. Fix an A-algebra $\mathbf{A}$ and an element $\hbar \in \mathrm{A}$ which is regular in $\mathbf{A}$. Let $\mathbf{A} \rightarrow \overline{\mathbf{A}}=\mathbf{A} / \hbar \mathbf{A}, a \mapsto \bar{a}$ be the canonical map. Assume that $\overline{\mathbf{A}}$ is a commutative ring. Fix $a_{1}, a_{2}, \ldots, a_{r} \in \mathbf{A}$ such that $\left(\bar{a}_{1}, \bar{a}_{2}, \ldots, \bar{a}_{r}\right)$ is a regular sequence in $\overline{\mathbf{A}}$. 
Let $\mathbf{I} \subset \mathbf{A}$ be a left ideal generated by $a_{1}, a_{2}, \ldots, a_{r}$. If $\tau_{i j} a_{j} a_{i}-a_{i} a_{j} \in \hbar \mathbf{I}$ with $\tau_{i j} \in 1+\hbar \mathrm{A}$, then $\hbar$ acts regularly on $\mathbf{A} / \mathbf{I}$.

Proof. Given an element $a \in \mathbf{A}$ such that $\hbar a \in \mathbf{I}$ we set $\hbar a=\sum_{i=1}^{r} b_{i} a_{i}$ with $b_{1}, \ldots, b_{r} \in \mathbf{A}$. We have $0=\sum_{i=1}^{r} \bar{b}_{i} \bar{a}_{i}$. Since $\left(\bar{a}_{1}, \bar{a}_{2}, \ldots, \bar{a}_{r}\right)$ is a regular sequence, we have $\bar{b}_{1}=\sum_{i=2}^{r} \bar{b}_{1 i} \bar{a}_{i}$ with $b_{12}, \ldots, b_{1 r} \in \mathbf{A}$. So $\left(\bar{b}_{2}+\bar{a}_{1} \bar{b}_{12}\right) \bar{a}_{2} \in \sum_{i=3}^{r} \overline{\mathbf{A}} \bar{a}_{i}$. Since $\left(\bar{a}_{2}, \bar{a}_{3}, \ldots, \bar{a}_{r}\right)$ is a regular sequence, we have $\bar{b}_{2}+\bar{a}_{1} \bar{b}_{12}=\sum_{i=3}^{r} \bar{b}_{2 i} \bar{a}_{i}$ with $b_{23}, \ldots, b_{2 r} \in \mathbf{A}$. In this way we construct inductively an element $b_{i j} \in \mathbf{A}$ for each $i<j$ such that

$$
\bar{b}_{i}=\sum_{j>i} \bar{b}_{i j} \bar{a}_{j}-\sum_{j<i} \bar{b}_{j i} \bar{a}_{j} .
$$

Now fix $c_{1}, \ldots, c_{r} \in \mathbf{A}$ such that

$$
b_{i}=\sum_{j>i} \tau_{i j} b_{i j} a_{j}-\sum_{j<i} b_{j i} a_{j}+\hbar c_{i} .
$$

We have

$$
\hbar\left(a-\sum_{i} c_{i} a_{i}\right)=\sum_{j>i} b_{i j}\left(\tau_{i j} a_{j} a_{i}-a_{i} a_{j}\right) \in \hbar \mathbf{I} .
$$

Thus $\hbar a \in \hbar \mathbf{I}$. Since $\hbar$ is regular in $\mathbf{A}$, we have $a \in \mathbf{I}$. Thus for each $a^{\prime} \in \mathbf{A} / \mathbf{I}$ we have $a^{\prime}=0$ whenever $\hbar a^{\prime}=0$, i.e., $\hbar$ acts regularly on $\mathbf{A} / \mathbf{I}$.

A.11.3. Lemma. Let $H$ be a split reductive group over $\mathbb{Z}$. Let A, B be commutative finitely generated $H$-rings. Let $\varphi: \mathrm{A} \rightarrow \mathrm{B}$ be a surjective $H$-equivariant ring homomorphism. There is a Zariski open subset $U \subset \operatorname{Spec}(\mathbb{Z})$ such that for every closed point $U \rightarrow \mathrm{k}$ the induced morphism $(\mathrm{A} \otimes \mathrm{k})^{H} \rightarrow(\mathrm{B} \otimes \mathrm{k})^{H}$ is again surjective.

Proof. The torsion subgroup of B is a B-module of finite type. Thus there is an integer $r \neq 0$ such that for each $\mathbb{Z}_{r} \rightarrow \mathrm{k}$, $\mathrm{k}$ a field, we have $\operatorname{Tor}_{1}^{\mathbb{Z}}(\mathrm{B}, \mathrm{k})=0$. Set $M=\operatorname{Ker}(\varphi)$. There is an exact sequence

$$
0 \rightarrow M \otimes \mathrm{k} \rightarrow \mathrm{A} \otimes \mathrm{k} \rightarrow \mathrm{B} \otimes \mathrm{k} \rightarrow 0 .
$$

Since $M$ is an A-module of finite type there is an integer $s \neq 0$ such that if $\mathbb{Z}_{s} \rightarrow \mathrm{k}$, then $H^{1}(H, M \otimes \mathrm{k})=0$. See, for instance, [CV, Theorem B.3]. Thus if $\mathbb{Z}_{r s} \rightarrow \mathrm{k}$, then we have an exact sequence

$$
0 \rightarrow(M \otimes \mathrm{k})^{H} \rightarrow(\mathrm{A} \otimes \mathrm{k})^{H} \rightarrow(\mathrm{B} \otimes \mathrm{k})^{H} \rightarrow 0 .
$$

\section{A.12. Proof of 2.4.}

Proof of 2.4.3. (a) Recall that $\mathbf{V}=\mathbf{U}^{\prime} / \mathbf{I}_{V}$. Let $\mathcal{V} \subset \mathbf{V}$ be the image of $\mathcal{U}^{\prime}$. The map $\mathfrak{z}_{U^{\prime}} \circ \iota: \mathcal{O}(G) \rightarrow \mathcal{U}^{\prime}$ yields a $G_{\pi}$-algebra isomorphism

$$
\mathcal{O}(G) /\left(\bar{c}_{i j}-\delta_{i j} ; j \neq 1\right) \rightarrow \mathcal{V} .
$$

The lhs is an isomorphic localization of the subalgebra of $\mathcal{O}(G)$ generated by $\left\{\bar{c}_{i 1}\right\}$ at $\bar{c}_{11}$. So there is a $G_{\pi}$-algebra isomorphism

$$
\mathcal{O}\left(\mathbb{A}_{\diamond}^{n}\right) \rightarrow \mathcal{O}(G) /\left(\bar{c}_{i j}-\delta_{i j} ; j \neq 1\right), v_{i}+\delta_{i 1} \mapsto \bar{c}_{i 1} .
$$

Thus the map (A.8.4),

$$
\mathfrak{z}_{D}: \mathcal{O}(D)=\mathcal{O}(G) \otimes \mathcal{O}(G) \rightarrow \mathcal{D},
$$

factors to a $G_{\pi} \times G$-algebra isomorphism

$$
\mathfrak{z}_{R}: \mathcal{O}(R)=\mathcal{O}(G) \otimes \mathcal{O}\left(\mathbb{A}_{\diamond}^{n}\right) \rightarrow \mathcal{R} .
$$


Now, we must prove that there is an algebra isomorphism

$$
\mathcal{O}\left(\bar{R}_{\pi}\right) \rightarrow \mathcal{O}(R)^{G_{\pi}}
$$

It is enough to prove that the algebra homomorphism

$$
\tau: \mathcal{O}\left(T^{*} \mathbb{A}^{n}\right) \rightarrow \mathcal{O}\left(G \times \mathbb{A}^{n}\right)^{G_{\pi}}, \quad v_{i} \mapsto \sum_{j} \iota\left(c_{v_{i}, \varphi_{j}}\right) \otimes v_{j}, \varphi_{i} \mapsto c_{v_{1}, \varphi_{i}} \otimes 1
$$

is invertible, because $\tau\left(\sum_{i} v_{i} \varphi_{i}\right)=1 \otimes v_{1}$. To prove this, observe first that $\tau$ is invertible over $\mathrm{k}$. Next, note that $\tau$ is a graded homomorphism and each graded part is an A-module of finite type. Therefore, to prove that $\tau$ is invertible it is enough to check that

$$
\mathcal{O}\left(G \times \mathbb{A}^{n}\right)^{G_{\pi}} \otimes \mathrm{k}=\mathcal{O}\left(G_{\mathrm{k}} \times \mathbb{A}_{\mathrm{k}}^{n}\right)^{G_{\pi, \mathrm{k}}} .
$$

This follows by a standard argument with good filtrations.

Finally, we must check that the map

$$
G \times \mathbb{A}^{n} \rightarrow\left\{(v, \varphi) \in T^{*} \mathbb{A}^{n} ; \varphi \neq 0\right\}, \quad(g, v) \mapsto\left(g^{-1} v, \varphi_{1} g\right)
$$

is a $G_{\pi}$-torsor, where $G_{\pi}$ acts on the lhs as in (2.4.1). This is left to the reader.

(b) The first claim is obvious, because $\partial_{\triangleright}\left(\mathcal{U}^{\prime}\right) \subset \mathcal{R}^{\pi}$. The algebra homomorphisms

$$
\partial_{\triangleright \mathfrak{z}_{U^{\prime}}}: \mathcal{O}(G) \rightarrow \mathcal{D}, \quad \mu_{R}: \mathcal{O}(G) \rightarrow \mathcal{R}^{\pi}
$$

and the canonical maps $\mathcal{D} \rightarrow \mathcal{R}, \mathcal{R}^{\pi} \rightarrow \mathcal{R}$ give the commutative square

$$
\begin{array}{ccc}
\mathcal{O}(G) & \rightarrow & \mathcal{D} \\
\downarrow & & \downarrow \\
\mathcal{R}^{\pi} & \rightarrow & \mathcal{R} .
\end{array}
$$

Since $\mathfrak{z}_{F}$ is a Hopf algebra homomorphism (A.5.1) and (A.8.4) yield

$$
\partial_{\triangleright} \mathfrak{z}_{U^{\prime}}(f)=\mathfrak{z}_{D}\left(\iota\left(f_{1}\right) f_{3} \otimes \iota\left(f_{2}\right)\right), \quad \forall f .
$$

Thus the upper arrow is the comorphism of the map $D \rightarrow G,(g, h) \mapsto g^{-1} h^{-1} g$. Furthermore, the right one and the lower one are the comorphisms of the maps

$$
\begin{aligned}
& R \rightarrow D,(g, v) \mapsto\left(g, e+v \otimes \varphi_{1}\right), \\
& R \rightarrow \bar{R}_{\pi},(g, v) \mapsto\left(g^{-1} v, \varphi_{1} g\right) .
\end{aligned}
$$

(c) Let $\mathcal{F}^{\pi},{ }^{\pi} \mathcal{F} \subset \mathbf{F}$ be the subalgebras generated by $\left\{\left(c_{1 i}\right)^{l}\right\},\left\{\left(c_{i 1}\right)^{l}\right\}$. Note that $\mathbf{V},{ }^{\pi} \mathbf{F}$ are free of rank $l^{n}$ over $\mathcal{V},{ }^{\pi} \mathcal{F}$ and that $\mathcal{V}$ is the localization of ${ }^{\pi} \mathcal{F}$ at $\left(c_{11}\right)^{l}$. Identify $\mathbf{R}$ with $\mathbf{F} \otimes \mathbf{V}$ and $\mathcal{R}$ with $\mathcal{F} \otimes \mathcal{V}$. Then $\mathbf{R}$ is a free $\mathcal{R}$-module. Similarly, $\mathbf{R}^{\pi}$ is a localization of $\mathbf{R}_{+}^{\pi}$, we have $\mathbf{R}_{+}^{\pi} \simeq \mathbf{F}^{\pi} \otimes{ }^{\pi} \mathbf{F}$ and $\mathbf{F}^{\pi},{ }^{\pi} \mathbf{F}$ are free of rank $l^{n}$ over $\mathcal{F}^{\pi},{ }^{\pi} \mathcal{F}$. This yields the second assertion.

Proof of 2.4.4. The open sets

$$
R_{\pi, i}=\left\{(v, \varphi) \in \bar{R}_{\pi} ; \varphi_{i} \neq 0\right\}
$$

form an affine open cover of $R_{\pi}$. We claim that $\mathfrak{R}\left(R_{\pi, i}\right)$ is an $\mathcal{O}\left(R_{\pi, i}\right)$-module of finite type for all $i$. Note that

$$
\mathcal{O}\left(R_{\pi, i}\right)=\mathcal{R}_{i}^{\pi}, \quad \mathfrak{R}\left(R_{\pi, i}\right)=\mathbf{R}_{i}^{\pi},
$$

are the local rings of $\mathcal{R}^{\pi}, \mathbf{R}^{\pi}$ at $\left(c_{1 i}\right)^{l}, c_{1 i}$. Furthermore, $\mathbf{D}_{i}^{\pi}$ is a $\mathcal{D}_{i}^{\pi}$-module of finite type and the natural map $\mathbf{D}_{i}^{\pi} \rightarrow \mathbf{R}_{i}^{\pi}$ is surjective by $2.3 .4(b),(d)$. Hence the claim is obvious. 
Now we set $\mathrm{A}=\mathbb{C}$. We have $\partial_{\triangleright}\left(\mathbf{U}^{\prime}\right) \subset \mathbf{R}^{\pi}$ by $2.2 .3(a)$ and

$$
\mathfrak{z}_{U^{\prime}}(\Sigma)=\left\{k_{\lambda} ; \lambda \in 2 l X_{+}\right\} .
$$

Thus $\mathfrak{R}\left(R_{\pi, \Sigma}\right)=\mathbf{R}_{\Sigma}^{\pi}$. It is a quantum torus by 1.9.1(b). So it is an Azumaya algebra.

\section{A.13. Proof of 2.5.}

Proof of 2.5.6. (a) See the proof of 2.4.4.

(b) For each $i$ let

$$
S_{\pi, i}=D \times R_{\pi, i}, \quad R_{\pi, i}=\left\{(v, \varphi) \in \bar{R}_{\pi} ; \varphi_{i} \neq 0\right\} .
$$

The sets $S_{\pi, i}$ form an affine open cover of $S_{\pi}$. Thus we have

$$
\mathfrak{S}_{\pi}\left(S_{\pi}\right)=\bigcap_{i} \mathbf{S}_{i}^{\pi}
$$

Hence there is a canonical inclusion $\mathbf{S}^{\pi} \rightarrow \mathfrak{S}_{\pi}\left(S_{\pi}\right)$. To prove the first claim it is enough to check that this map is invertible. We have $\mathbf{F}=\bigcap_{i} \mathbf{F}_{i}$. Thus we have $\mathbf{S}=\bigcap_{i} \mathbf{S}_{i}$. The claim follows. Next, the $\dot{\mathbf{U}}$-module

$$
\mathfrak{S}_{\pi}\left(S_{\pi, s}\right)=\mathbf{S}_{s}^{\pi}
$$

is locally finite for each $s$. Thus the $\dot{\mathbf{U}}$-module $\mathfrak{S}_{\pi}\left(S_{\pi, \diamond}\right)$ is also locally finite.

$(c)$ Set $\mathrm{A}=\mathbb{C}$. By 2.4.5(b) and A.17.4 the set $S_{\pi, \diamond}(\mathbb{C})$ consists of the tuples $\left(g, g^{\prime}, v, \varphi\right)$ such that

$$
\operatorname{det}\left(\varphi m_{1}, \varphi m_{2}, \ldots, \varphi m_{n}\right) \neq 0
$$

where $m_{1}, m_{2}, \ldots, m_{n}$ are noncommutative monomials in $g, g^{\prime}$. This yields the second claim. The first one is obvious. The last claim follows from the second one.

(d) Set $\mathrm{A}=\mathbb{C}$. We have $\mathfrak{S}_{\pi}\left(S_{\pi, \Sigma}\right)=\mathbf{S}_{\Sigma}^{\pi}$. By 2.2.6(b) the map $\psi$ yields an A-linear isomorphism

$$
\mathbf{R}_{\Sigma}^{\pi} \otimes \mathbf{D}_{\Sigma}^{\prime} \rightarrow \mathbf{S}_{\Sigma}^{\pi}
$$

By A.7.4(c) there is a positive filtration of $\mathbf{S}_{\Sigma}^{\pi}$ such that this isomorphism is multiplicative up to lower terms where, in the left-hand side, homogeneous elements in $\mathbf{R}_{\Sigma}^{\pi}, \mathbf{D}_{\Sigma}^{\prime}$ may only quasi-commute with each other. Thus $\mathbf{S}_{\Sigma}^{\pi}$ is an Azumaya algebra over $S_{\pi, \Sigma}$ by 2.2.4, 2.4.4 and standard filtered/graded techniques; see VV1.

(e) Set $\mathrm{A}=\mathbb{C}$. First, we prove that $\mathcal{O}_{S_{\pi}}=Z\left(\mathfrak{S}_{\pi}\right)$. Recall the affine open cover

$$
S_{\pi}=\bigcup_{i} S_{\pi, i}
$$

introduced in (A.13.1). By (2.5.3) we have an algebra embedding

$$
\mathcal{O}\left(S_{\pi, i}\right) \subset \mathbf{S}_{i}^{\pi} .
$$

Note that $\mathbf{S}^{\pi}$ is an ID by 2.2.6(e). Therefore $Z\left(\mathbf{S}_{i}^{\pi}\right)$ is a CID and a $\mathcal{O}\left(S_{\pi, i}\right)$-module of finite type. Furthermore, we have

$$
\operatorname{Frac}\left(\mathcal{O}\left(S_{\pi, i}\right)\right)=\operatorname{Frac}\left(Z\left(\mathbf{S}_{i}^{\pi}\right)\right),
$$

because $\mathbf{S}_{\Sigma}^{\pi}$ is an Azumaya algebra over $S_{\pi, \Sigma}$ by part $(d)$. Finally, $\mathcal{O}\left(S_{\pi, i}\right)$ is an ICD. So we have

$$
\mathcal{O}\left(S_{\pi, i}\right)=Z\left(\mathbf{S}_{i}^{\pi}\right) .
$$

Hence we have

$$
\mathcal{O}_{S_{\pi}}=Z\left(\mathfrak{S}_{\pi}\right) .
$$


We have $\mathbb{C}=\mathcal{A} /(q-\tau)$ with $\mathcal{A}=\mathbb{C}\left[q^{ \pm 1}\right]$. Furthermore, $\mathbf{S}_{i}^{\pi}=\mathbb{S}_{\mathcal{A}, i}^{\pi} \otimes \mathbb{C}$ by 2.3.4 $(c)$. Setting $\hbar=l\left(q^{l}-q^{-l}\right)$ in A.10.2 we get a Poisson bracket on $\mathcal{O}\left(S_{\pi, i}\right)$ for each $i$ by (A.13.3). These Poisson brackets glue together, yielding a Poisson bracket on

$$
\mathcal{O}\left(\bar{S}_{\pi}\right)=\bigcap_{i} \mathcal{O}\left(S_{\pi, i}\right)
$$

Next, the coaction of $G$ on $\bar{S}_{\pi}$ is a Poisson algebra homomorphism

$$
\mathcal{O}\left(\bar{S}_{\pi}\right) \rightarrow \mathcal{O}(G) \otimes \mathcal{O}\left(\bar{S}_{\pi}\right)
$$

because it is the specialization of the coaction map

$$
\mathbb{S}_{\mathcal{A}}^{\pi} \rightarrow \mathbb{F}_{\mathcal{A}} \otimes \mathbb{S}_{\mathcal{A}}^{\pi}
$$

and the latter is an $\mathcal{A}$-algebra homomorphism since $\mathbb{S}_{\mathcal{A}}^{\pi}$ is a $\dot{U}_{\mathcal{A}}$-algebra. So the $G$-action on $\bar{S}_{\pi}$ is a Poisson action, i.e., $\bar{S}_{\pi}$ is a Poisson $G$-variety.

Now we prove the second claim. The sets $S_{\pi, i}$ form an affine open cover of $S_{\pi}$. Furthermore, $\mathbf{S}_{i}^{\pi}$ is a Poisson order over $S_{\pi, i}$ for each $i$ by A.10.2(b), (A.13.3). Thus $\mathfrak{S}_{\pi}$ is a Poisson order over $S_{\pi}$.

Finally, we prove the last claim. We must check that for each $s \in \mathcal{O}\left(\bar{S}_{\pi}\right)^{+}$which is homogeneous of positive degree the algebra $\mathbf{S}_{(s)}^{\pi}=\left(\mathbf{S}_{s}^{\pi}\right)^{0}$ is a Poisson order over $\mathcal{O}\left(S_{\pi, s}\right)^{0}$. Recall that the superscript 0 means Ü-invariants; see (2.3.2). Since $\mathfrak{S}_{\pi}$ is a Poisson order over $S_{\pi}$ we have that $\mathbf{S}_{s}^{\pi}$ is a Poisson order over $S_{\pi, s}$ by (A.13.2). Thus the claim is proved by taking invariants.

\section{A.14. Proof of 3.4.}

Proof of 3.4.6(a). The left $\mathbf{H}$-action on $\mathbf{H}$ o yields a ring homomorphism

$$
\eta: \mathbf{H} \rightarrow \operatorname{End}_{\mathbf{S H}}(\mathbf{H} o) .
$$

We have $\operatorname{End}_{\mathbf{S H}}(\mathbf{H} o)_{\star}=\operatorname{End}_{\mathbf{S H}_{\star}}\left(\mathbf{H}_{\star} O\right)$. Thus, under localization the map $\eta$ yields a ring homomorphism

$$
\eta_{\star}: \mathbf{H}_{\star}=\mathbf{D}_{0, \star} \rtimes \Sigma_{n} \rightarrow \operatorname{End}_{\mathbf{D}_{0, \star}^{\Sigma_{n}}}\left(\mathbf{D}_{0, \star}\right) .
$$

This map is invertible (left to the reader). Thus, since $\mathbf{H} \subset \mathbf{H}_{\star}$ the map $\eta$ is injective. The cokernel of $\eta$ is a torsion $\mathcal{L}$-module. Using $\mathfrak{F}_{H}$ one shows easily that it is supported on a closed subset of $\operatorname{Spec}(\mathcal{L})$ of codimension $\geqslant 2$. Recall that the $\mathcal{L}$-module $\mathbf{H}$ is free, and that $\operatorname{End}_{\mathbf{S H}}(\mathbf{H} o)$ is a torsion-free $\mathcal{L}$-module of finite type, because it embeds in the free $\mathcal{L}$-module of finite type $\operatorname{End}_{\mathcal{L}}(\mathbf{H} o)$. Thus the map $\eta$ is invertible by 3.4.4.

Next, we must prove that the inclusion $Z(\mathbf{H}) \circ \subset Z(\mathbf{S H})$ is an equality. The right action of an element $z \in Z(\mathbf{S H})$ on $\mathbf{H} o$ lies in $\operatorname{End}_{\mathbf{S H}}(\mathbf{H} o)$. Hence it is equal to $\eta(x)$ for some $x \in \mathbf{H}$. In particular, we have

$$
x^{\prime} x o=x^{\prime} o z=x x^{\prime} o, \quad \forall x^{\prime} \in \mathbf{H} .
$$

Hence we have also

$$
x^{\prime} x x^{\prime \prime} o=x^{\prime} x^{\prime \prime} x o=x x^{\prime} x^{\prime \prime} o, \quad \forall x, x^{\prime} \in \mathbf{H} .
$$

This implies that

$$
\eta([x, \mathbf{H}])=0 .
$$

Thus we have $x \in Z(\mathbf{H})$, because $\eta$ is invertible. So we have $z=a_{o}^{-1} x o \in Z(\mathbf{H}) o$. 
A.15. Proof of 3.5. Assume that $a_{o}$ is invertible. For each integer $r>0$ recall the finite subset $X_{r} \subset X$ from section A.9. Let $F_{r}\left(\mathbb{H}_{\mathrm{A}}\right) \subset \mathbb{H}_{\mathrm{A}}$ be the A-submodule spanned by the elements $x_{\lambda} t_{w} y_{\lambda^{\prime}}$ with $\left(\lambda, w, \lambda^{\prime}\right) \in X_{r} \times \Sigma_{n} \times X_{r}$. Put

$$
F_{r}\left(\mathbb{S H}_{\mathrm{A}}\right)=\mathbb{S H H}_{\mathrm{A}} \cap F_{r}\left(\mathbb{H}_{\mathrm{A}}\right) \text {. }
$$

A.15.1. Lemma. (a) The A-module $F_{r}\left(\mathbb{H}_{\mathrm{A}}\right)$ is a direct summand of $\mathbb{H}_{\mathrm{A}}$ of finite type.

(b) We have $F_{r}\left(\mathbb{H}_{\mathrm{A}}\right) \cdot F_{s}\left(\mathbb{H}_{\mathrm{A}}\right) \subset F_{r+s}\left(\mathbb{H}_{\mathrm{A}}\right)$.

(c) The A-module $F_{r}\left(\mathbb{S H}_{\mathrm{A}}\right)$ is a direct summand of $\mathbb{S H}_{\mathrm{A}}$ of finite type.

Proof. (a) Obvious by (3.1.1).

(b) Let $F_{r}^{x}\left(\mathbb{H}_{\mathrm{A}}\right), F_{r}^{y}\left(\mathbb{H}_{\mathrm{A}}\right) \subset \mathbb{H}_{\mathrm{A}}$ be the A-submodules spanned by the elements $x_{\lambda} t_{w} y_{\lambda^{\prime}}$ with $\left(\lambda, w, \lambda^{\prime}\right) \in X_{r} \times \Sigma_{n} \times X, X \times \Sigma_{n} \times X_{r}$ respectively. We have

$$
F_{r}\left(\mathbb{H}_{\mathrm{A}}\right)=F_{r}^{x}\left(\mathbb{H}_{\mathrm{A}}\right) \cap F_{r}^{y}\left(\mathbb{H}_{\mathrm{A}}\right) \text {. }
$$

Thus it is enough to prove that

$$
F_{r}^{x}\left(\mathbb{H}_{\mathrm{A}}\right) \cdot F_{s}^{x}\left(\mathbb{H}_{\mathrm{A}}\right) \subset F_{r+s}^{x}\left(\mathbb{H}_{\mathrm{A}}\right), \quad F_{r}^{y}\left(\mathbb{H}_{\mathrm{A}}\right) \cdot F_{s}^{y}\left(\mathbb{H}_{\mathrm{A}}\right) \subset F_{r+s}^{y}\left(\mathbb{H}_{\mathrm{A}}\right) .
$$

We have $X_{r}+X_{s} \subset X_{r+s}$. Thus to prove the first inclusion it is enough to check that $t_{w} x_{\lambda} \in F_{r}^{x}\left(\mathbb{H}_{\mathrm{A}}\right)$ for each $(\lambda, w) \in X_{r} \times \widetilde{\Sigma}_{n}$. The second inclusion is identical. We may assume that $w=s_{i}$, a simple affine reflection. Recall that

$$
t_{i} x_{\lambda}=x_{s_{i} \lambda} t_{i}+\left(\zeta-\zeta^{-1}\right)\left(x_{\lambda}-x_{s_{i} \lambda}\right) /\left(1-x_{\alpha_{i}}\right) .
$$

Thus we have

$$
t_{i} x_{\lambda} \in \bigoplus_{j \in J} x_{\lambda+j \alpha_{i}} \mathbb{H}_{\mathrm{A}}^{y}
$$

where $J=\left\{0,1, \ldots,-\alpha_{i} \cdot \lambda\right\}$ if $\alpha_{i} \cdot \lambda \leqslant 0$ and $J=\left\{-\alpha_{i} \cdot \lambda, \ldots,-2,-1\right\}$ otherwise. We also have $\lambda+j \alpha_{i} \in X_{r}$ for each $(\lambda, j) \in X_{r} \times J$. So $t_{i} x_{\lambda} \in F_{r}^{x}\left(\mathbb{H}_{\mathrm{A}}\right)$.

(c) Since $o \in F_{0}\left(\mathbb{H}_{\mathrm{A}}\right)$ we have $o F_{r}\left(\mathbb{H}_{\mathrm{A}}\right) o \subset F_{r}\left(\mathbb{S H}_{\mathrm{A}}\right)$ by part $(b)$. Inversely, there are inclusions $F_{r}\left(\mathbb{S H}_{\mathrm{A}}\right) \subset o F_{r}\left(\mathbb{S H}_{\mathrm{A}}\right) o \subset o F_{r}\left(\mathbb{H}_{\mathrm{A}}\right) o$ because $o x o=a_{o}^{2} x$ for all $x \in \mathbb{S H}_{\mathrm{A}}$. Thus we have

$$
F_{r}\left(\mathbb{S H}_{\mathrm{A}}\right)=o F_{r}\left(\mathbb{H}_{\mathrm{A}}\right) o .
$$

So $F_{r}\left(\mathbb{S H}_{\mathrm{A}}\right)$ is a direct summand in $F_{r}\left(\mathbb{H}_{\mathrm{A}}\right)$, hence in $\mathbb{H}_{\mathrm{A}}$, hence in $\mathbb{S H}_{\mathrm{A}}$.

Let $\mathbb{S H}_{\mathrm{A}}^{\prime} \subset \mathbb{S H}_{\mathrm{A}}$ be the A-subalgebra generated by $o \mathbb{X}_{\mathrm{A}}^{\Sigma_{n}}$ and $o \mathbb{Y}_{\mathrm{A}}^{\Sigma_{n}}$. For each integers $a, b$ we set $p_{a, b}=\sum_{i} x_{i}^{a} y_{i}^{b}$. Let $F_{r}\left(\mathbb{S H}_{\mathrm{A}}^{\prime}\right) \subset \mathbb{S H}_{\mathrm{A}}^{\prime}$ be the A-submodule spanned by the elements of the form

$$
o x_{\omega_{n}}^{-a} y_{\omega_{n}}^{-b} p_{a_{1}, 0} p_{0, b_{1}} p_{a_{2}, 0} \cdots,
$$

where $a, a_{i}, b, b_{i} \geqslant 0$ are integers such that $r \geqslant \sum_{i} a_{i} \geqslant a$ and $r \geqslant \sum_{i} b_{i} \geqslant b$.

A.15.2. Lemma. Let $\mathrm{A}$ be the localization of $\mathbb{C}\left[q^{ \pm 1}, t^{ \pm 1}\right]$ at the multiplicative set generated by $q^{2}-1, \ldots, q^{2 n}-1$ and $a_{o}$. We have

(a) $\mathbb{S H}_{\mathrm{A}}=\mathbb{S H}_{\mathrm{A}}^{\prime}+\left(t^{2}-1\right) \mathbb{S H}_{\mathrm{A}}$,

(b) $F_{r}\left(\mathbb{S H}_{\mathrm{A}}\right)=F_{r}\left(\mathbb{S H}_{\mathrm{A}}^{\prime}\right)+\left(t^{2}-1\right) F_{r}\left(\mathbb{S H}_{\mathrm{A}}\right)$.

Proof. (a) Let $\mathbf{A}$ be the A-algebra consisting of the $\Sigma_{n}$-invariant elements in the quantum torus generated by $\mathbf{x}_{i}, \mathbf{x}_{i}^{-1}, \mathbf{y}_{i}, \mathbf{y}_{i}^{-1}$ modulo the relations

$$
\begin{gathered}
\mathbf{x}_{i} \mathbf{x}_{i}^{-1}=\mathbf{x}_{i}^{-1} \mathbf{x}_{i}=1, \quad \mathbf{y}_{i} \mathbf{y}_{i}^{-1}=\mathbf{y}_{i}^{-1} \mathbf{y}_{i}=1, \\
\mathbf{x}_{i} \mathbf{y}_{j}=q^{2 \delta_{i j}} \mathbf{y}_{j} \mathbf{x}_{i}, \quad \mathbf{x}_{i} \mathbf{x}_{j}=\mathbf{x}_{j} \mathbf{y}_{i}, \quad \mathbf{y}_{i} \mathbf{y}_{j}=\mathbf{y}_{j} \mathbf{y}_{i} .
\end{gathered}
$$


There is an obvious A-algebra isomorphism

$$
\mathrm{SHH}_{\mathrm{A}} /\left(t^{2}-1\right) \rightarrow \mathbf{A} \text {. }
$$

It takes $\mathbb{S H H}_{\mathrm{A}}^{\prime} /\left(t^{2}-1\right)$ onto the subalgebra $\mathbf{A}^{\prime} \subset \mathbf{A}$ generated by the symmetric monomials in the $\mathbf{x}_{i}$ 's and the symmetric monomials in the $\mathbf{y}_{i}$ 's. We must prove that $\mathbf{A}^{\prime}=\mathbf{A}$.

For each integers $a, b$ we set

$$
\mathbf{p}_{a, b}=\sum_{i} \mathbf{x}_{i}^{a} \mathbf{y}_{i}^{b} .
$$

A classical argument due to $\mathrm{H}$. Weyl implies that the A-algebra $\mathbf{A}$ is generated by the set $\left\{\mathbf{p}_{a, b} ; a, b \in \mathbb{Z}\right\}$. We have

$$
\left(1-q^{-2 a}\right) \mathbf{p}_{a, b}=\left[\mathbf{p}_{a, b-1}, \mathbf{p}_{0,1}\right] .
$$

Thus an induction on $b$ shows that $\mathbf{p}_{a, b} \in \mathbf{A}^{\prime}$ for each $a=1,2, \ldots, n$ and $b \geqslant 0$. The case $b \leqslant 0$ is identical. Now we have

$$
\mathbf{p}_{a, b}=\sum_{i=1}^{n}(-1)^{i-1} \mathbf{x}_{\omega_{i}} \mathbf{p}_{a-i, b},
$$

where $\mathbf{x}_{\omega_{i}}$ is the $i$ th elementary symmetric function. So $\mathbf{p}_{a, b} \in \mathbf{A}^{\prime}$ for each $a, b \in \mathbb{Z}$.

(b) We have $F_{r}\left(\mathbb{S H H}_{\mathrm{A}}^{\prime}\right) \subset F_{r}\left(\mathbb{S H}_{\mathrm{A}}\right)$. Further, the proof in $(a)$ implies that

$$
F_{r}\left(\mathbb{S H}_{\mathrm{A}}\right) \subset F_{r}\left(\mathbb{S H}_{\mathrm{A}}^{\prime}\right)+\left(t^{2}-1\right) \mathbb{S H}_{\mathrm{A}} .
$$

Finally, A.15.1(c) yields

$$
\left(t^{2}-1\right) \mathbb{S H}_{\mathrm{A}} \cap F_{r}\left(\mathbb{S H H}_{\mathrm{A}}\right)=\left(t^{2}-1\right) F_{r}\left(\mathbb{S H}_{\mathrm{A}}\right) .
$$

Thus we have $F_{r}\left(\mathbb{S H}_{\mathrm{A}}\right) \subset F_{r}\left(\mathbb{S H}_{\mathrm{A}}^{\prime}\right)+\left(t^{2}-1\right) \mathbb{S H}_{\mathrm{A}}$. The other inclusion is obvious.

Proof 3.5.1. Let us assume temporarily that $\alpha$ is as in A.15.2. Note that

$$
M_{\mathrm{A}}=\mathbb{S H}_{\mathrm{A}} / \mathbb{S H}_{\mathrm{A}}^{\prime}
$$

is a $\mathbb{X}_{\mathrm{A}}^{\Sigma_{n}} \otimes \mathbb{Y}_{\mathrm{A}}^{\Sigma_{n}}$-module of finite type by (3.1.1), with $\mathbb{X}_{\mathrm{A}}^{\Sigma_{n}}$ acting by left multiplication and $\mathbb{Y}_{\mathrm{A}}^{\Sigma_{n}}$ by right multiplication. Thus the support of the $\alpha$-module $M_{\mathrm{A}}$ is a closed subset $S \subset \operatorname{Spec}($ A). By A.15.2(b) we have

$$
F_{r}\left(M_{\mathrm{A}}\right)=\left(t^{2}-1\right) F_{r}\left(M_{\mathrm{A}}\right), \quad \forall r .
$$

Since localization commutes with direct limits, this yields

$$
S \subset\left\{t^{2} \neq 1\right\} .
$$

Now consider the open set

$$
U=\left\{(\tau, \zeta) \in \Gamma_{c}(\mathbb{C}) ; \tau^{2}, \tau^{4}, \ldots, \tau^{2 n} \neq 1, a_{o} \neq 0\right\} .
$$

Since $k>2 n$ there is a point $(\varepsilon, 1) \in U$. So $U \not \subset S(\mathbb{C})$. Since the curve $\Gamma_{c}$ is irreducible the set $S(\mathbb{C}) \cap U$ is finite. Hence $(\tau, \zeta) \notin S(\mathbb{C})$ if $l$ is large enough. Thus $M_{\mathcal{A}_{b}}=0$. Therefore we have

$$
\mathbb{S H H}_{\mathcal{A}_{b}}=\mathbb{S H H}_{\mathcal{A}_{b}}^{\prime} \text {. }
$$

In particular, under the assumptions in 3.5.1 we get an algebra isomorphism

$$
\mathbf{S H}=\mathbf{S H}^{\prime} .
$$


The proposition is now obvious. Set $\mathrm{A}=\mathbb{C}$. By (1.11.3) we have algebra homomorphisms

$$
L^{\prime}: Z\left(\mathbf{F}^{\prime}\right) \rightarrow \mathbf{D}_{0, \star}^{\Sigma_{n}}, \quad L: Z\left(\mathbf{U}^{\prime}\right) \rightarrow \mathbf{D}_{0, \star}^{\Sigma_{n}} .
$$

By definition of $\Phi$ we have inclusions

$$
L^{\prime}\left(Z\left(\mathbf{F}^{\prime}\right)\right), L\left(Z\left(\mathbf{U}^{\prime}\right)\right) \subset \Phi\left(\mathbf{T}^{0}\right) .
$$

Furthermore, it is well known that

$$
\Psi(o f)=L^{\prime}(f), \quad \Psi(o u)=L(u), \quad \forall f \in \mathbf{X}^{\Sigma_{n}}, u \in \mathbf{Y}^{\Sigma_{n}} ;
$$

see $[\mathrm{C}$, [K2, Theorem 5.9]. Therefore we have

$$
\Psi(\mathbf{S H})=\Psi\left(\mathbf{S H}^{\prime}\right) \subset \Phi\left(\mathbf{T}^{0}\right) .
$$

\section{A.16. Proof of 3.6.}

Proof of 3.6.1. The functor

$$
F^{*}: \operatorname{Mod}(\mathbf{A}) \rightarrow \operatorname{Mod}(o \mathbf{A} o), M \mapsto o M
$$

is exact. Its right adjoint is given by

$$
F_{*}(N)=\operatorname{Hom}_{o \mathbf{A} o}(o \mathbf{A}, N) .
$$

There is an isomorphism $F^{*} \circ F_{*} \simeq \operatorname{id}_{\mathcal{M} \boldsymbol{o d}(o \mathbf{A} o)}$. So $\operatorname{Mod}(\mathbf{A}, o)=\operatorname{Ker}\left(F^{*}\right)$ is a localizing subcategory and $F^{*}$ factors to an equivalence

$$
\boldsymbol{M} \boldsymbol{\operatorname { c o d }}(\mathbf{A}) / \operatorname{Ker}\left(F^{*}\right) \rightarrow \boldsymbol{M} \boldsymbol{M o d}(o \mathbf{A} o)
$$

by [G, prop. III.2.5].

Proof of 3.6.5(a). First, let us give more notation. Let $\mathfrak{b} \subset \mathfrak{s l}_{\mathrm{R}}$ be the Iwahori Lie subalgebra associated with the set of positive roots $\Pi_{+}$; see section 1.1. Let $\mathcal{B}$ be the set of Lie subalgebras in $\mathfrak{s l}_{\mathrm{F}}$ which are $S L(\mathrm{~F})$-conjugate to $\mathfrak{b}$. Let $\pi$ be the unique morphism

$$
\pi: \mathcal{B} \rightarrow \mathcal{G}, \quad(\operatorname{ad} g)(\mathfrak{b}) \mapsto(\operatorname{ad} g)\left(\mathfrak{s l}_{\mathrm{R}}\right) .
$$

The automorphism $a=a_{s, \tau, \zeta}$ in (3.6.3) yields an automorphism of $\mathcal{B}$. The fixed points subset, $\mathcal{B}^{a}$, is a disjoint union of smooth connected $\widetilde{S L}(\mathrm{~F})^{a}$-varieties. We write

$$
\mathcal{B}^{a}=\bigcup_{i \in \Xi} \mathcal{B}_{i}^{a} .
$$

Next, we set

$$
\begin{aligned}
{ }^{1} \dot{\mathcal{N}}^{a} & =\left\{(x, \mathfrak{p}) \in \mathcal{N}^{a} \times \mathcal{B}^{a} ; x \in \mathfrak{p}_{+}\right\}, \\
{ }^{1} \dot{\mathcal{N}}_{i}^{a} & ={ }^{1} \dot{\mathcal{N}}^{a} \cap\left(\mathcal{N}^{a} \times \mathcal{B}_{i}^{a}\right) .
\end{aligned}
$$

Also, let ${ }^{12} \ddot{\mathcal{N}}_{i j}^{a},{ }^{1} \ddot{\mathcal{N}}_{i j}^{a},{ }^{2} \ddot{\mathcal{N}}_{i j}^{a}$ denote the set of triples $\left(x, \mathfrak{p}, \mathfrak{p}^{\prime}\right)$ with, respectively,

$$
x \in \mathfrak{p}_{+} \cap \mathfrak{p}_{+}^{\prime} \cap \mathcal{N}^{a}, \quad\left(\mathfrak{p}, \mathfrak{p}^{\prime}\right) \in \mathcal{B}_{i}^{a} \times \mathcal{B}_{j}^{a}, \mathcal{B}_{i}^{a} \times \mathcal{G}_{j}^{a}, \mathcal{G}_{i}^{a} \times \mathcal{B}_{j}^{a} .
$$

Consider the vector spaces

$$
\mathbb{K}=\prod_{j} \bigoplus_{i} K\left({ }^{12} \ddot{\mathcal{N}}_{i j}^{a}\right), \quad{ }^{1} \mathbb{P}=\prod_{j} \bigoplus_{i} K\left({ }^{1} \ddot{\mathcal{N}}_{i j}^{a}\right), \quad{ }^{2} \mathbb{P}=\prod_{j} \bigoplus_{i} K\left({ }^{2} \ddot{\mathcal{N}}_{i j}^{a}\right) .
$$

Recall that the convolution product in $K$-theory, denoted $*$, yields $\mathbb{C}$-algebra structures on $\mathbb{K}, \mathbb{S K}$; see (3.6.4). It also yields the linear maps

$$
\text { * : }{ }^{2} \mathbb{P} \otimes \mathbb{K} \otimes{ }^{1} \mathbb{P} \rightarrow \mathbb{S} \mathbb{K}, \quad *:{ }^{1} \mathbb{P} \otimes \mathbb{S K} \otimes{ }^{2} \mathbb{P} \rightarrow \mathbb{K} .
$$


See [V] for details. Put $o=\prod_{i, j} o_{i j},{ }^{12} o=\prod_{i, j}{ }^{12} o_{i j}$, etc., where

$$
o_{i j} \in \mathbb{S} \mathbb{K}, \quad{ }^{12} o_{i j} \in \mathbb{K}, \quad{ }^{1} o_{i j} \in{ }^{1} \mathbb{P} \quad{ }^{2} o_{i j} \in{ }^{2} \mathbb{P}
$$

are the fundamental classes of the varieties

$$
\begin{aligned}
& \left\{\left(x, \mathfrak{p}, \mathfrak{p}^{\prime}\right) ; \mathfrak{p}=\mathfrak{p}^{\prime}\right\}, \quad\left\{\left(x, \mathfrak{p}, \mathfrak{p}^{\prime}\right) ; \pi(\mathfrak{p})=\pi\left(\mathfrak{p}^{\prime}\right)\right\} \\
& \left\{\left(x, \mathfrak{p}, \mathfrak{p}^{\prime}\right) ; \pi(\mathfrak{p})=\mathfrak{p}^{\prime}\right\}, \quad\left\{\left(x, \mathfrak{p}, \mathfrak{p}^{\prime}\right) ; \pi\left(\mathfrak{p}^{\prime}\right)=\mathfrak{p}\right\} .
\end{aligned}
$$

We define the linear maps

$$
\begin{array}{ll}
\alpha: \mathbb{K} \rightarrow \mathbb{S K}, & x \mapsto{ }^{2} o * x *{ }^{1} o, \\
\beta: \mathbb{S K} \rightarrow \mathbb{K}, & x \mapsto{ }^{1} o * x *{ }^{2} o .
\end{array}
$$

The unit of $\mathbb{S K}$ for $*$ is $1_{S K}=o$. The unit of $\mathbb{K}$ for $*$ is the product of the fundamental classes of the varieties $\left\{\left(x, \mathfrak{p}, \mathfrak{p}^{\prime}\right) ; \mathfrak{p}=\mathfrak{p}^{\prime}\right\}$. We will denote it by $1_{K}$.

Further, it is proved in $[\mathrm{V}$, thm. $4.9(i)$, sec. 6.1.1] that there is a $\mathbb{C}$-algebra homomorphism

$$
\Psi: \mathbb{H}_{\mathbb{C}} \rightarrow \mathbb{K}
$$

which is continuous with a dense image. Here $\mathbb{K}$ is given the finite topology as in section 3.6. A routine computation yields the following lemma (left to the reader).

\section{A.16.1. Lemma. The following relations hold:}

(a) we have $\alpha\left(1_{K}\right)=\gamma 1_{S K}$ for some $\gamma \in \mathbb{C}^{\times}$, and $\beta\left(1_{S K}\right)={ }^{12} o$,

(b) we have $\Psi\left(\mathbb{S H}_{\mathbb{C}}\right)={ }^{12} \mathrm{o} * \Psi\left(\mathbb{H}_{\mathbb{C}}\right) *{ }^{12}$ o.

From A.16.1( $a)$ we obtain

$$
\begin{aligned}
& \alpha \beta(x)=\alpha\left(1_{K}\right) * x * \alpha\left(1_{K}\right)=\gamma^{2} x, \quad \forall x \in \mathbb{S K}, \\
& \beta \alpha(x)=\beta\left(1_{S K}\right) * x * \beta\left(1_{S K}\right)={ }^{12} o * x *{ }^{12} o, \quad \forall x \in \mathbb{K} .
\end{aligned}
$$

So $\beta$ factors to an isomorphism

$$
\beta: \mathbb{S K} \rightarrow{ }^{12} o * \mathbb{K} *{ }^{12} o .
$$

Thus, by A.16.1(b) the map $\Psi$ factors to a continuous algebra homomorphism

$$
\mathrm{S} \Psi: \mathbb{S H}_{\mathbb{C}} \rightarrow \mathbb{S K}
$$

with a dense image. So the pullback of a smooth simple $\mathbb{S K}$-module by $\mathrm{S} \Psi$ is a simple $\mathbb{S H}_{\mathbb{C}}$-module. It yields a map

$$
\left.\{\text { smooth simple } \mathbb{S K} \text {-modules }\} \rightarrow \text { simple } \mathbb{S H}_{\mathbb{C}}-\text { modules }\right\} .
$$

The injectivity is obvious; see the proof of [V, thm. 4.9(iv)].

\section{A.17. Proof of 4.1 .}

Proof of 4.1.1(b). Let us prove that $T_{\pi,+}$ is an integral complete intersection of dimension $2 n+n^{2}$. Let $G_{+, \star} \subset G_{+}$be the set of diagonalizable matrices with eigenvalues $h_{1}, h_{2}, \ldots, h_{n}$ such that $\zeta^{2 l} h_{j} \neq h_{i}$ for each $i, j$ and $h_{j} \neq h_{i}$ for $i \neq j$. Let $T_{\pi,+, \star} \subset T_{\pi,+}$ be the set of tuples $\left(h, g^{\prime}, v, \varphi\right)$ such that $h \in G_{+, \star}$. Set $H_{+, \star}=$ $G_{+, \star} \cap H$. Each $G$-orbit in $T_{\pi,+, \star}$ contains a tuple of the form $\left(h, g^{\prime}, v, \varphi\right)$ with $h \in H_{+, \star}$ and $g^{\prime}$ a matrix with $(i, j)$ th entry $v_{i} \varphi_{j} /\left(\zeta^{2 l} h_{j}-h_{i}\right)$. So we have

$$
T_{\pi,+, \star} \simeq\left(H_{+, \star} \times T^{*} \mathbb{A}^{n}\right) \times_{H} G .
$$

It is smooth, connected and of dimension $2 n+n^{2}$. 
Since $T_{\pi,+}$ is given by $n^{2}$ equations, each irreducible component has dimension $\geqslant 2 n+n^{2}$. We claim that the closed subset

$$
F=T_{\pi,+} \backslash T_{\pi,+, \star}
$$

has dimension $<2 n+n^{2}$. Thus $T_{\pi,+}$ is irreducible of dimension $2 n+n^{2}$. Further, it is generically reduced and a complete intersection. So it is reduced.

Now, we concentrate on the claim. Let $J_{k}(a)$ be the Jordan block of size $k$ and eigenvalue $a$. Let $m$ be the order of $\zeta^{2 l}$. Note that $m \neq 1$. Fix $g \in G_{+}$of the form

$$
g=\bigoplus_{a=1}^{r} g_{a}, \quad g_{a}=\bigoplus_{s \in \mathbb{Z} / m} \bigoplus_{i=1}^{i_{a, s}} J_{k_{a, s},}\left(\lambda_{a} \zeta^{2 s l}\right) .
$$

Here we have $\lambda_{a} \notin \lambda_{b} \zeta^{2 l \mathbb{Z}}$ if $a \neq b, i_{a, s}$ is an integer $\geqslant 0$ and $k_{a, s}=\left\{k_{a, s, i} ; i=\right.$ $\left.1,2, \ldots, i_{a, s}\right\}$ is a decreasing sequence of integers $>0$. Set $n_{a, s}=\sum_{i} k_{a, s, i}$ and $n_{a}=\sum_{a, s} n_{a, s}$ for all $a$. We will assume that $n_{a} \neq 0$ and that $n_{a, s}=0$ if $\lambda_{a}=0$ and $s \neq 0$. Note that $g \notin G_{+, \star}$ iff at least one of the following conditions holds:

- $\lambda_{a}=0$ for some $a$,

- $n_{a, s}, n_{a, s-1} \neq 0$ for some $a, s$,

- $n_{a, s}>1$ for some $a, s$.

The decomposition $g=\bigoplus_{a=1}^{r} g_{a}$ yields a partition of $\mathbb{C}^{n}, \mathbb{C}^{n, *}$ into $r$ subspaces. For each $v \in \mathbb{C}^{n}, \varphi \in \mathbb{C}^{n, *}$ let $v=\sum_{a} v(a), \varphi=\sum_{a} \varphi(a)$ be the corresponding decomposition. We have also a decomposition of each matrix $A \in G_{+}$as a sum of blocs $A(a, b)$. In the same way we define the bloc $A(a, s, i, b, t, j)$. Let $a_{x, y}^{\nu}$ be the $(x, y)$ th entry of the bloc $A(\nu)$. We may abbreviate $a_{x, y}=a_{x, y}^{\nu}$ when the bloc is clear from the context. As usual we set $a_{x, y}^{a, s, i, b, t, j}=0$ if $y<1, x<1, x>k_{a, s, i}$ or $y>k_{b, t, j}$.

For $g \in G_{+}, z \in \mathbb{C}$ we put $\mathrm{k}_{g, z}=\operatorname{Ker}\left(Z_{g, z}\right), \mathrm{I}_{g, z}=\Im\left(Z_{g, z}\right)$ where

$$
Z_{g, z}: G_{+} \rightarrow G_{+}, g^{\prime} \mapsto g g^{\prime}-z^{2 l} g^{\prime} g
$$

Also, set

$$
\mathrm{V}_{g, \zeta}=\left\{(v, \varphi) ; v \otimes \varphi \in \mathrm{I}_{g, \zeta}\right\}
$$

Consider the map

$$
q: T_{\pi,+} \rightarrow G_{+},\left(g, g^{\prime}, v, \varphi\right) \mapsto g
$$

We have $Z_{g, \zeta}\left(g^{\prime}\right)+v \otimes \varphi=0$ for each $\left(g, g^{\prime}, v, \varphi\right) \in T_{\pi,+}$. Thus for $g \in G_{+}$we have

$$
\operatorname{dim} q^{-1}(g) \leqslant \operatorname{dim}\left(\mathrm{V}_{g, \zeta}\right)+\operatorname{dim}\left(\mathrm{k}_{g, \zeta}\right) .
$$

Therefore we also have $\operatorname{dim} q^{-1}(G g) \leqslant n^{2}+d(g)$ where

$$
d(g)=\operatorname{dim}\left(\mathrm{V}_{g, \zeta}\right)+\operatorname{dim}\left(\mathrm{k}_{g, \zeta}\right)-\operatorname{dim}\left(\mathrm{k}_{g, 1}\right) .
$$

Recall that we must prove that $\operatorname{dim}(F)<2 n+n^{2}$.

For each $r=1,2, \ldots, n$ let $G(r) \subset G_{+}$be the set of matrices which are conjugated to a matrix $\bigoplus_{a=1}^{r} g_{a}$ as above. Given $g \in G(r)$ we compute $d(g)$ as in $\mathrm{O}$, sec. 2.3].

First we assume that $r=1$, i.e., we have $g=g_{a}, n=n_{a}$. Note that for each $w \in \Sigma_{n}$ the set of tuples $(v, \varphi)$ such that the matrix $B=v \otimes \varphi$ satisfies $b_{x, w(x)}=0$ for all $x$ has dimension $n$. The following holds:

- Assume that $\lambda_{a}=0$. We have $d(g)=\operatorname{dim}\left(\mathrm{V}_{g, \zeta}\right)$ by $\mathrm{A} .17 .1(b)$. If $(v, \varphi) \in$ $\mathrm{V}_{g, \zeta}$, the diagonal entries of $v \otimes \varphi$ vanish by A.17.1(c). So $d(g) \leqslant n \leqslant 2 n-1$. 
- Assume that $\lambda_{a} \neq 0$ and either $n_{a, s}=0$ or $n_{a, s-1}=0$ for each $s$. We have $d(g)<\operatorname{dim}\left(\mathrm{V}_{g, \zeta}\right)$ by (A.17.2). Hence $d(g) \leqslant 2 n-1$. Furthermore, if $n_{a, s}>1$ for some $s$, then either $k_{a, s, 1} \geqslant 2$ or $i_{a, s} \geqslant 2$, and in both cases we have $d(g)<\operatorname{dim}\left(\mathrm{V}_{g, \zeta}\right)-1$ by (A.17.2), so $d(g)<2 n-1$.

- Assume that $\lambda_{a} \neq 0$ and $n_{a, s}, n_{a, s-1} \neq 0$ for some $s$. The equations in A.17.1(c) are nontrivial. So we have $\operatorname{dim}\left(V_{g, \zeta}\right)<2 n$. Thus $d(g) \leqslant 2 n-1$. Furthermore, $d(g)<2 n-1$ if $k_{a, s} \neq k_{a, t}$ for some $t$ by A.17.1(b).

- Assume that $\lambda_{a} \neq 0$ and $k_{a, s}=k_{a, t}$ for each $s, t$. Since $m>1$ we have also $n>1$. Choose $s=t+1$. We have $d(g)=\operatorname{dim}\left(\mathrm{V}_{g, \zeta}\right)$ by A.17.1(b). Given $(v, \varphi) \in \mathrm{V}_{g, \zeta}$ we put $B=v \otimes \varphi$. By A.17.1(c) the diagonal entries of $B(a, s, a, t)$ vanish. Thus we have $\operatorname{dim}(g) \leqslant n<2 n-1$.

Now let $r$ be arbitrary. By A.17.1 $(a)$ we have $d(g) \leqslant \sum_{a} d\left(g_{a}\right)$. The discussion above implies that $d\left(g_{a}\right) \leqslant 2 n_{a}-1$ for all $a$. Further, the equality may only occur if

- $\lambda_{a}=0$ and $n_{a}=1$,

- $\lambda_{a} \neq 0$, either $n_{a, s}=0$ or $n_{a, s-1}=0$ for each $s$, and $n_{a, s} \leqslant 1$ for each $s$.

In the second case we have necessarily $g \in G_{+, \star}$. So if $g \in G(r) \cap\left(G_{+} \backslash G_{+, \star}\right)$, then $d(g) \leqslant 2 n-r$ and the equality occurs only if $g \notin G$. For each $a$ the matrix $g_{a}$ has only one continuous paramater if $\lambda_{a} \neq 0$, and 0 otherwise. So we have

$$
\operatorname{dim}\left(q^{-1} G(r) \cap F\right)<2 n+n^{2}, \quad \forall r .
$$

We are done.

\section{A.17.1. Lemma.}

(a) We have $d(g) \leqslant \sum_{a} d\left(g_{a}\right)$.

(b) If $r=1$, then $\operatorname{dim}\left(\mathrm{k}_{g, \zeta}\right) \leqslant \operatorname{dim}\left(\mathrm{k}_{g, 1}\right)$ with the equality iff either $\lambda_{a}=0$ or $k_{a, s}=k_{a, t}$ for each $s, t$.

(c) If $r=1$ and $A \in \mathrm{I}_{g, \zeta}$ has rank one, then $a_{x, y}^{a, s, i, a, t, j}=0$ if $y-x \leqslant$ $\min \left\{0, k_{a, t, j}-k_{a, s, i}\right\}$ and either $\lambda_{a}=0$ or $s=t+1$.

Proof. (a) First we claim that

$$
\mathrm{k}_{g, \zeta}=\bigoplus_{a} \mathrm{k}_{g_{a}, \zeta}, \quad \mathrm{k}_{g, 1}=\bigoplus_{a} \mathrm{k}_{g_{a}, 1} .
$$

We have $A \in \mathrm{k}_{g, \zeta}$ iff

$$
J_{k_{a, s, i}}\left(\lambda_{a} \zeta^{2 s l}\right) A(a, s, i, b, t, j)=\zeta^{2 l} A(a, s, i, b, t, j) J_{k_{b, t, j}}\left(\lambda_{b} \zeta^{2 t l}\right), \quad \forall a, s, i, b, t, j .
$$

These equations are equivalent to

$$
a_{x, y}\left(\lambda_{a} \zeta^{2 s l}-\lambda_{b} \zeta^{2(t+1) l}\right)=\zeta^{2 l} a_{x, y-1}-a_{x+1, y}, \forall x, y, a, s, i, b, t, j
$$

where the superscripts $a, s, i, b, t, j$ are omitted. These equations are also equivalent to the following:

- if $a \neq b$ or $\left(a=b, \lambda_{a} \neq 0, s \neq t+1\right)$, then $A(a, s, i, b, t, j)=0$,

- otherwise $a_{x+1, y}=\zeta^{2 l} a_{x, y-1}$ for each $x=1,2, \ldots, k_{a, s, i}, y=1,2, \ldots, k_{b, t, j}$ (in particular, we have $a_{x, y}=0$ if $x-y>\min \left\{0, k_{a, s, i}-k_{b, t, j}\right\}$ ).

This implies the first claim. The second one is identical.

Now, the assignment $(v, \varphi) \mapsto(v(a), \varphi(a))$ maps $\mathrm{V}_{g, \zeta}$ into $\mathrm{V}_{g_{a}, \zeta}$ because

$$
A=Z_{g, \zeta}\left(g^{\prime}\right) \Rightarrow A(a, a)=Z_{g_{a}, \zeta}\left(g^{\prime}(a, a)\right) .
$$

Thus we have $\operatorname{dim}\left(\mathrm{V}_{g, \zeta}\right) \leqslant \sum_{a} \operatorname{dim}\left(\mathrm{V}_{g_{a}, \zeta}\right)$. We are done. 
(b) Set $r=1$. For each $u$ we put $h_{a, s, u}=\sharp\left\{i ; k_{a, s, i} \geqslant u\right\}$. If $\lambda_{a} \neq 0$, the equations above imply that $\operatorname{dim}\left(\mathrm{k}_{g, \zeta}\right)=\sum_{s, i, j} \min \left\{k_{a, s, i}, k_{a, s-1, j}\right\}$. Hence we have

$$
\begin{aligned}
\operatorname{dim}\left(\mathrm{k}_{g, \zeta}\right)-\operatorname{dim}\left(\mathrm{k}_{g, 1}\right) & =\sum_{s, i, j}\left(\min \left\{k_{a, s, i}, k_{a, s-1, j}\right\}-\min \left\{k_{a, s, i}, k_{a, s, j}\right\}\right) \\
& =\sum_{s} \sum_{u \geqslant 1} h_{a, s, u}\left(h_{a, s-1, u}-h_{a, s, u}\right) \\
& =-\sum_{s} \sum_{u \geqslant 1}\left(h_{a, s, u}-h_{a, s-1, u}\right)^{2} / 2 .
\end{aligned}
$$

If $\lambda_{a}=0$, they yield $\operatorname{dim}\left(\mathrm{k}_{g, \zeta}\right)=\operatorname{dim}\left(\mathrm{k}_{g, 1}\right)=\sum_{i, j} \min \left\{k_{a, 0, i}, k_{a, 0, j}\right\}$.

(c) Set $r=1$. Note that $A \in \mathrm{I}_{g, \zeta}$ iff $\operatorname{tr}(A B)=0$ for all $B \in \mathrm{k}_{g, \zeta^{-1}}$.

Assume first that $\lambda_{a} \neq 0$. For each triple $(a, s, i)$ we have

$$
\sum_{b, t, j} \operatorname{tr}(A(a, s, i, b, t, j) B(b, t, j, a, s, i))=0, \forall B \in \mathrm{k}_{g, \zeta^{-1}}
$$

iff we have

$$
\sum_{e=1}^{k_{a, s, i}} \zeta^{-2 l e} a_{e, y+e}^{a, s, i, b, t, j}=0
$$

if $y \leqslant \min \left\{0, k_{b, t, j}-k_{a, s, i}\right\}, a=b$ and either $s=t+1$ or $\lambda_{a}=\lambda_{b}=0$. The claim follows, because the lowest nonzero diagonal of a rank one matrix contains only one nonzero entry.

If $\lambda_{a}=0$, we get the equations

$$
\sum_{e=1}^{k_{a, 0, i}} \zeta^{-2 l e} a_{e, y+e}^{a, 0, i, a, 0, j}=0
$$

for all $i, j$ and all $y \leqslant \min \left\{0, k_{a, 0, j}-k_{a, 0, i}\right\}$. We are done.

Proof of 4.1.2. (a) Fix a tuple $x=\left(h, g^{\prime}, v, \varphi\right) \in T_{\pi, \star}$. We may assume that $h \in H_{\star}$. The equation $m_{+}(x)=0$ implies that the $(i, j)$ th entry of $g^{\prime}$ is

$$
v_{i} \varphi_{j} /\left(\zeta^{2 l} h_{j}-h_{i}\right) \text {. }
$$

Since the matrix $g^{\prime}$ is invertible this yields $v, \varphi \in H$. Set $h^{\prime}=v \varphi / h$ and $g=\varphi / h$. We have $g x=x_{h, h^{\prime}}$. So $T_{\pi, \star}$ consists of all tuples in $T_{\pi}$ whose $G$-orbit contains a representative in $\left\{x_{h, h^{\prime}} ; h \in H_{\star}, h^{\prime} \in H\right\}$. Thus the assignment $\left(h, h^{\prime}\right) \mapsto x_{h, h^{\prime}}$ yields an isomorphism

$$
\left(H_{\star} \times H\right) / \Sigma_{n} \rightarrow T_{\pi, \star} / G=N_{\star} .
$$

We have $T_{\pi, \star} \subset T_{\pi, \diamond}$ and $\mathfrak{F}_{T}\left(T_{\pi, \diamond}^{\prime}\right)=T_{\pi, \diamond}$. Let us check that $T_{\pi, \star} \subset T_{\pi, \diamond}$. Fix $h, h^{\prime}$ as above. Any subspace of $\mathbb{C}^{n, *}$ containing $h$ and is stable by the $h$-action contains $h^{m}$ for all $m \in \mathbb{Z}$. Thus $\mathbb{C}^{n, *}$ is the only subspace with these properties, because $h_{i} \neq h_{j}$ if $i \neq j$. Hence $x_{h, h^{\prime}} \in T_{\pi, \diamond}$ by 2.5.6(c).

(b) It is easy to see that $m_{+}$is a submersion at each point of $T_{\pi, \diamond}$. So $T_{\pi, \diamond}$ is smooth. The freeness of the $G$-action follows from 2.5.6(c).

The proof that $\mathfrak{q}$ yields an isomorphism $T_{\star} \rightarrow N_{\star}$ is easy. It suffices to note that if $x \in T_{\pi, \star}$, then we may assume that $x=x_{h, h^{\prime}}$ for some $h, h^{\prime}$ by part $(a)$. Then the claim is routine. 
Now, let us concentrate on the second claim. We have $\mathcal{O}(N)=\mathcal{O}\left(T_{\pi}\right)^{0}$. By A.17.4 the algebra $\mathcal{O}(N)$ is generated by the set

$$
\left\{(\operatorname{det} m)^{ \pm 1}, \operatorname{tr}(m), \varphi(m v) ; m\right\} \text {. }
$$

Here $m$ runs over all monomials in $g, g^{\prime}$. Since $m_{S}=\zeta^{2 l} e$ over $T_{\pi}$, it is indeed generated by the subset

$$
\left\{(\operatorname{det} m)^{ \pm 1}, \operatorname{tr}(m) ; m\right\} \text {. }
$$

So the pullback by the map $T_{\pi} \subset \bar{S}_{\pi} \rightarrow D,\left(g, g^{\prime}, v, \varphi\right) \mapsto\left(g, g^{\prime}\right)$ yields a surjective algebra homomorphism

$$
\mathcal{O}(D)^{0} \rightarrow \mathcal{O}(N)
$$

The Poisson bracket on $\mathcal{O}(N)$ is the restriction of the Poisson bracket on $\mathcal{O}\left(T_{\pi}\right)$. By 2.2.3 $(e)$ we have $\mathcal{O}(D)=Z\left(\mathbf{D}^{\prime}\right)$. Thus A.10.2 yields a Poisson bracket on $D$. The inclusion $\mathcal{O}(D) \subset \mathcal{O}\left(\bar{S}_{\pi}\right)$ is a Poisson homomorphism. See the proof of 2.5.6(d). So the map (A.17.3) is a Poisson algebra homomorphism. Thus, to compute the Poisson bracket on $N$ it is enough to compute the bracket on $D$. To do so we may assume that $l=1$. By 1.8.3 we have

$$
\begin{aligned}
& \left\{L_{1}, L_{2}\right\}=R_{21}^{c} L_{1} L_{2}-L_{1} L_{2} R_{12}^{c}+L_{1} R_{12}^{c} L_{2}-L_{2} R_{21}^{c} L_{1}, \\
& \left\{L_{1}^{\prime}, L_{2}^{\prime}\right\}=R_{21}^{c} L_{1}^{\prime} L_{2}^{\prime}-L_{1}^{\prime} L_{2}^{\prime} R_{12}^{c}+L_{1}^{\prime} R_{12}^{c} L_{2}^{\prime}-L_{2}^{\prime} R_{21}^{c} L_{1}^{\prime}, \\
& \left\{L_{1}, L_{2}^{\prime}\right\}=-R_{21}^{c} L_{1} L_{2}^{\prime}-L_{1} L_{2}^{\prime} R_{21}^{c}+L_{2}^{\prime} R_{21}^{c} L_{1}-L_{1} R_{12}^{c} L_{2}^{\prime},
\end{aligned}
$$

where $R^{c}$ is the classical $R$-matrix corresponding to $R^{q}$. These are exactly the formulas found in [FR, A.2-A.4] (up to a sign).

A.17.4. Lemma. Let A be a field of characteristic 0 or large enough. The Aalgebra $\mathcal{O}\left(D \times T^{*} \mathbb{A}^{n}\right)^{+}$is generated by

$$
\left\{\operatorname{det}\left(\varphi m_{1}, \varphi m_{2}, \ldots, \varphi m_{n}\right), \operatorname{det}(m)^{ \pm 1}, \operatorname{tr}(m), \varphi(m v)\right\}
$$

where $m, m_{i}$ are monomials in $g, g^{\prime}$. Further, the natural map $\mathcal{O}\left(D \times \mathbb{A}^{n, *} \times G\right)^{+} \rightarrow$ $\mathcal{O}\left(T_{\pi}\right)^{+}$is surjective.

Proof. Since a field extension is faithfully flat, it is enough to assume that $\alpha$ is an algebraically closed field. Then the first claim is well known. Let us concentrate on the second one. Recall the homomorphism

$$
\begin{aligned}
& \mathcal{O}\left(D \times \mathbb{A}^{n, *} \times G\right) \rightarrow \mathcal{O}\left(D \times T^{*} \mathbb{A}^{n}\right), \\
& f \otimes w \otimes 1 \mapsto f \otimes 1 \otimes w, \\
& f \otimes 1 \otimes c_{\psi, w} \mapsto \psi(w) f \otimes 1 \otimes 1+f \otimes \psi \otimes w .
\end{aligned}
$$

The induced map $\mathcal{O}\left(D \times \mathbb{A}^{n, *} \times G\right)^{+} \rightarrow \mathcal{O}\left(D \times T^{*} \mathbb{A}^{n}\right)^{+}$is surjective by the first part of the lemma. The restriction $\mathcal{O}\left(D \times T^{*} \mathbb{A}^{n}\right) \rightarrow \mathcal{O}\left(T_{\pi}\right)$ is also surjective. Thus, if the characteristic of $\mathrm{A}$ is large enough, we have a surjection by A.11.3,

$$
\mathcal{O}\left(D \times \mathbb{A}^{n, *} \times G\right)^{+} \rightarrow \mathcal{O}\left(T_{\pi}\right)^{+} .
$$

Proof of 4.1.3. Recall that $\mathcal{O}(N)=\mathcal{T}^{0}$ by 2.6.2(a). Thus the maps $z, z^{\prime}$ in (1.11.2) yield an algebra homomorphism $\mathcal{L} \rightarrow \mathcal{O}(N)$. See the proof of 3.4.1. A routine computation shows that this map equips $\mathcal{O}(N)$ with the structure of an $\mathcal{L}$-module of finite type. 


\section{A.18. Proof of 4.2 .}

Proof of 4.2.2. First, note that $M$ is a torsion-free B-module, because $M \subset M \otimes \mathrm{k}$ and $\mathrm{B} \subset \mathrm{B} \otimes \mathrm{k}$. Further, $M \otimes \mathrm{k}$ and $M \otimes \mathrm{k}$ are locally free over $\mathrm{B} \otimes \mathrm{k}$ and $\mathrm{B} \otimes \mathrm{k}$ respectivelly, because they are flat and the $\mathrm{B}$-module $M$ is finitely presented. So it is enough to check that both ranks coincide. Let $\mathrm{B}^{\prime}$ be the localization of $\mathrm{B}$ at the generic point of $\operatorname{Spec}(\mathrm{B} \otimes \mathrm{k})$. It is a DVR. Further, the $\mathrm{B}^{\prime}$-module $M \otimes_{\mathrm{B}} \mathrm{B}^{\prime}$ is finitely generated and torsion-free. Thus $M \otimes_{\mathrm{B}} \mathrm{B}^{\prime}$ is a free $\mathrm{B}^{\prime}$-module. This implies our claim.

Proof of 4.2.4. (a) Serre's functor $\mathcal{Q g r}(\mathrm{A}) \rightarrow \mathcal{Q} \operatorname{coh}\left(\mathcal{O}_{X}\right)$ is an equivalence of categories which takes $\mathrm{B}$ to the sheaf of $\mathcal{O}_{X}$-algebras $\mathcal{E}$. Thus it yields an equivalence of categories $\mathcal{Q} \operatorname{gr}(\mathrm{B}) \rightarrow \mathcal{Q} \operatorname{coh}(\mathcal{E})$ such that $\mathrm{B} \mapsto \mathcal{E}$. There is also an equivalence of categories $\mathcal{Q} \operatorname{gr}(\mathrm{B}) \rightarrow \mathcal{Q} \operatorname{coh}\left(\mathcal{O}_{Y}\right)$ such that $\mathrm{B} \mapsto \mathcal{O}_{Y}$. Therefore we have graded ring isomorphisms

$$
H^{\bullet}\left(Y, \mathcal{O}_{Y}\right)=\operatorname{Ext}_{\mathcal{O}_{Y}}\left(\mathcal{O}_{Y}, \mathcal{O}_{Y}\right)=\operatorname{Ext}_{\mathcal{E}}^{\bullet}(\mathcal{E}, \mathcal{E})
$$

So the claim follows from the adjunction formula (i.e., Frobenius reciprocity), which gives

$$
\operatorname{Ext}_{\mathcal{E}}^{\bullet}(\mathcal{E}, \mathcal{E})=\operatorname{Ext}_{\mathcal{O}_{X}}^{\bullet}\left(\mathcal{O}_{X}, \mathcal{E}\right)=H^{\bullet}(X, \mathcal{E}) .
$$

(b) By A.18.2 we have $H^{>0}(X, \mathcal{E}) \otimes \mathrm{k}=0$. Since $\mathcal{E}$ is a coherent sheaf and $f$ is a proper morphism, for each $i$ we have the $\mathcal{O}\left(\bar{Y}_{i}\right)$-module of finite type

$$
H_{i}=H^{>0}(X, \mathcal{E}) \otimes_{\mathcal{O}(Y)} \mathcal{O}\left(\bar{Y}_{i}\right) .
$$

Note that

$$
H_{i} \otimes_{\alpha} \mathrm{k}=\left(H^{>0}(X, \mathcal{E}) \otimes \mathrm{k}\right) \otimes_{\mathcal{O}(Y \otimes \mathrm{k})} \mathcal{O}\left(\bar{Y}_{i} \otimes \mathrm{k}\right)=0 .
$$

Note also that $\mathcal{O}\left(\bar{Y}_{i}\right)$ is a domain and that there is a morphism $\mathcal{O}\left(\bar{Y}_{i}\right) \rightarrow \mathrm{k}$. Further a module of finite type over a CID which vanishes at some closed point is generically trivial by Nakayama's lemma. Thus we have

$$
H_{i} \otimes_{\mathcal{O}\left(\bar{Y}_{i}\right)} \mathrm{k}\left(\bar{Y}_{i}\right)=0 .
$$

On the other hand, $H^{\bullet}(X \otimes \mathbb{C}, \mathcal{E} \otimes \mathbb{C})$ is a Poisson order over $Y \otimes \mathbb{C}$ by A.10.3(b). Hence its restriction to $Y_{i} \otimes \mathbb{C}$ is locally free for each $i$ by A.10.3(a), thus it is zero by (A.18.1). Since $\left\{Y_{i} \otimes \mathbb{C}\right\}$ is a partition of $Y \otimes \mathbb{C}$ into locally closed subsets, this implies that $H^{>0}(X \otimes \mathbb{C}, \mathcal{E} \otimes \mathbb{C})=0$.

A.18.2. Lemma. Let A be a DVR with residue field $\mathrm{k}$. Let $X$ be a separated A-scheme of finite type and $\mathcal{E} \in \mathcal{Q} \operatorname{coh}\left(\mathcal{O}_{X}\right)$. If $\mathcal{E}$ is a flat A-module there is an exact sequence

$$
0 \rightarrow H^{\bullet}(X, \mathcal{E}) \otimes \mathrm{k} \rightarrow H^{\bullet}(X \otimes \mathrm{k}, \mathcal{E} \otimes \mathrm{k}) \rightarrow \operatorname{Tor}_{1}^{\mathrm{A}}\left(H^{\bullet+1}(X, \mathcal{E}), \mathrm{k}\right) \rightarrow 0 .
$$

Proof. Since $X$ is a Noetherian separated scheme, $H^{\bullet}(X, \mathcal{E})$ is the cohomology of the Cech complex of an affine open cover $X=\bigcup_{i} U_{i}$. Each term of the Cech complex is a flat A-module, because $\mathcal{E}$ is flat. Thus, the claim follows from the universal coefficient theorem; see [B2, X.4 cor. 1].

Proof of 4.2.7. Let us give a few details on the splitting of $\mathfrak{T}$ on the completion $\widehat{T}_{x}$ of $T$ along the subscheme $\mathfrak{q}^{-1}(x)$. The rest of the proof is as in BFG], BK1]. We must check that there is a vector bundle $\mathcal{V}_{x}$ on $\widehat{T}_{x}$ such that

$$
\left.\mathfrak{T}\right|_{\widehat{T}_{x}} \simeq \mathcal{E} n d_{\mathcal{O}_{\widehat{T}_{x}}} \mathcal{V}_{x}
$$


Morita equivalence classes of sheaves of Azumaya algebras on a scheme $X$ are classified by the Brauer group $\operatorname{Br}(X)$. We must check that the class [T of $\mathfrak{T}$ in $\operatorname{Br}(T)$ belongs to $\mathfrak{q}^{*}(B r(N))$, because the Brauer group of a local complete $\mathbb{C}$ algebra is trivial.

Recall that $\mathbf{D}_{0}$ is a $\Sigma_{n}$-equivariant Azumaya algebra over $\mathcal{D}_{0}$ (it is a quantum torus). Hence its class belongs to $\operatorname{Br}\left(\operatorname{Spec}\left(\mathcal{D}_{0}\right)\right)^{\Sigma_{n}}$. A theorem of Gabber implies that $\operatorname{Br}(X)$ is the torsion part of the étale cohomology group of the sheaf $\mathbb{G}_{m, X}$ if $X$ is affine. Since $\zeta^{2 l}=1$ we have $N \simeq \operatorname{Spec}\left(\mathcal{D}_{0}^{\Sigma_{n}}\right)$. So the norm-map associated to the projection $\mathfrak{r}: \operatorname{Spec}\left(\mathcal{D}_{0}\right) \rightarrow N$ gives a map

$$
\mathfrak{r}_{*}: \operatorname{Br}\left(\operatorname{Spec}\left(\mathcal{D}_{0}\right)\right)^{\Sigma_{n}} \rightarrow \operatorname{Br}(N) .
$$

Fix an element $0 \neq f \in \mathcal{L}$ such that $T_{f} \simeq N_{f}$ and $\mathfrak{T}\left(T_{f}\right)=\mathbf{T}_{f}^{0}$. We have proved that $\Phi$ yields algebra isomorphisms

$$
\mathfrak{T}\left(T_{f}\right) \simeq \mathbf{D}_{0, f}^{\Sigma_{n}}, \quad \mathcal{O}\left(T_{f}\right) \simeq \mathcal{D}_{0, f}^{\Sigma_{n}} .
$$

See the proof of 4.2.1(b). Therefore we have

$$
\left.\mathfrak{T}\right|_{T_{f}} \simeq \mathfrak{q}^{*}\left(\left.\mathfrak{r}_{*}\left(\mathbf{D}_{0}\right)\right|_{N_{f}}\right) .
$$

The restriction to a Zariski open dense subset induces an injective morphism of the corresponding Brauer groups. Thus we have

$$
[\mathfrak{T}]=\mathfrak{q}^{*} \mathfrak{r}_{*}\left[\widetilde{\mathbf{D}}_{0}\right] .
$$

\section{List OF NOTATIONS}

$1.1 X, X_{+}, Y, Y_{+}, \Pi, \Pi_{+}, G, H, U_{+}, U_{-}, \Sigma_{n}, \widehat{\Sigma}_{n}, \widetilde{\Sigma}_{n}, P, I, \delta$,

$1.2 \mathbf{A}_{\circ}, Z(\mathbf{A}), \mathcal{M o d}^{\mathrm{lf}}(\mathbf{A}), \mathbf{A}^{\chi}, \mathcal{G r}(\mathbf{A}), \boldsymbol{Q} \boldsymbol{g r}(\mathbf{A}), \widetilde{M}, \operatorname{lgd}(\mathbf{A}), \operatorname{fd}(M), \operatorname{pd}(M)$, $C I D, I D, N I D, C N I D, D V R, \bar{v}_{i}, \bar{\varphi}_{i}, e_{i j}$,

$1.3 \mathbf{H}^{\circ}, \mathbf{H}^{\varepsilon}, \mathbf{H}^{e}, \mathbf{H}^{\mathrm{lf}}, \mathbf{F}, \mathbf{U}^{[2]}, \mathbf{U}^{[3]}, \Lambda, r_{s}^{ \pm}, R^{ \pm}, \varpi_{2}, \varpi_{3}, \kappa, \bar{\kappa}$,

$1.4 \operatorname{Mod}(\mathbf{A}, \mathbf{H}), \mathbf{F}^{\prime}, \mathbf{A} \sharp \mathbf{H}, \mathbf{U}^{(2)}, \ell, \partial_{\triangleright}$,

$1.5 \mathbf{A} / /{ }_{\chi} \mathbf{H}^{\prime}, \mathbf{A} / / \mathbf{H}^{\prime}$

$1.6 X^{\prime}, \mathcal{K}, \mathbb{U}, \mathbb{U}_{ \pm}, \mathbb{U}_{0}, \mathfrak{a}, \mathfrak{a}^{\prime}, V(\lambda), M(\lambda), e_{i}, f_{i}, k_{\lambda}, k_{i}, v_{\lambda}, \varphi_{\lambda}, q^{\lambda}$,

$1.7 \mathbb{F}, \mathbb{F}^{\prime}, \mathbb{F}_{+}, \mathbb{F}_{+}^{\prime}, \mathbb{F}_{0}, \mathbb{F}_{\Sigma}, \mathbb{F}^{\pi}, \mathbb{U}^{\prime},\left(\mathbb{U}^{\prime}\right)^{[i]}, \mathbb{U}_{\Sigma}^{\prime}, \mathbb{U}_{\pi}, \mathbb{U}_{\pi}^{\prime}, \mathbb{U}_{\tilde{\pi}}, \mathbb{V}, R^{q}, \Sigma, \varrho_{0}, c_{\lambda}$, $c_{i j}, \bar{c}_{\lambda}, \bar{c}_{i j}, G_{\Sigma}$

$1.8 \mathbb{D}_{\triangleleft}, \mathbb{D}_{\triangleright}, \mathbb{D}, \mathbb{D}^{\prime}, \mathbb{D}_{+}, \mathbb{D}_{+}^{\prime},\left(\mathbb{U}^{\prime}\right)^{e}, \partial_{2}$,

$1.9 \mathbb{R}_{\triangleright}^{\pi}, \mathbb{R}^{\pi}, \mathbb{R}_{\Sigma}^{\pi}$

$1.10 \mathbb{H}, \mathbb{H}^{\prime}, \mathbb{G}, \mathbb{G}^{\pi}, \mathbb{E}, \mathbb{E}_{+}, \mathbb{E}_{\triangleright}, \mathbb{E}^{\pi}, \mathbb{S}, \mathbb{S}^{\pi}, \partial_{3}, \partial_{a}, \partial_{b}, \partial_{c}, \partial_{d}, \gamma, \gamma^{\prime}, \psi$,

$1.11 V \hat{\otimes} \mathbb{F}, I(V, W), \mathbb{D}_{0}, \mathbb{D}_{0, \star}, \mathbb{F}_{0, \star}, \mathbb{G}_{\chi}, \mathbb{T}^{0}, W, \Phi, L, \Omega, \nabla, \varrho, \pi, g_{\lambda}, \chi, a_{\mu}$,

$2.1 G^{*}, D, D^{*}, D_{\Sigma}, \bar{e}_{i}, \bar{f}_{i}, \bar{h}_{\lambda}$,

$2.2 \mathcal{A}, \mathrm{A}, \mathrm{k}, \mathrm{k}, \Gamma_{c}, \mathcal{A}_{c}, \mathrm{~A}_{c}, \mathrm{k}_{c}, \mathrm{k}_{c}, \mathrm{k}_{c}, \mathcal{A}_{b}, \mathrm{~A}_{b}, \mathrm{k}_{b}, \mathrm{k}_{b}, \mathbf{D}, \mathbf{F}, \mathbf{F}^{\prime}, \dot{\mathbf{U}}, \mathbf{U}, \mathbf{U}^{\prime}, \mathcal{D}$,

$\mathcal{F}, \mathcal{U}, \mathcal{U}^{\prime}, \mathfrak{z}_{D}, \mathfrak{z}_{U^{\prime}}, \mathfrak{z}_{F}, \mathfrak{a}, \mathfrak{s}_{0}, \mathfrak{s}, x_{\alpha}, y_{\alpha}, z_{\lambda}, c, k, m, l=p^{e}$,

$2.3 \mathbb{H}_{\mathrm{A}}^{\bullet}(\lambda), \mathbb{R}_{+, \mathrm{A}}^{\pi}, \mathbb{T}_{\mathrm{A}}$,

$2.4 \mathcal{R}, R, R_{\pi}, \bar{R}_{\pi}, R_{\pi, \Sigma}, \mathfrak{R}, \mathbf{V}, \mathcal{V}, \mathbb{A}_{\diamond}^{n}, m_{R}, G_{\pi}$,

$2.5 \mathcal{S}, \mathcal{S}^{\pi}, S, \bar{S}_{\pi}, S_{\pi}, S_{\pi, \diamond}, \mathfrak{S}_{\pi}$,

$2.6 \mathcal{S}_{(s)}^{\pi}, \mathcal{T}, T, T_{t}, T_{\pi}, N, m_{S}, \mathfrak{q},\left[g, g^{\prime}\right]$,

$2.7 \mathbf{T}, \mathbf{T}^{\mathbf{u}}, T_{\pi, \diamond}, \mathfrak{T}, \mathfrak{T}_{\pi}, \mathfrak{p}$,

$3.1 \mathbb{H}, \mathbb{S H}, o, \mathfrak{F}_{H}, x_{\lambda}, y_{\lambda}$,

$3.3 \Psi, \Psi^{\prime}$, 
$3.4 \mathcal{L}, C M, \sigma_{x}, \sigma_{y}$,

$3.6 \mathbb{S K K}_{\mathrm{A}}, \widetilde{S L}(\mathrm{~F})$,

$4.1 H_{\star}, G_{\star}, T_{\pi, \Sigma}, T_{\pi, \star}, T_{\pi, \odot}, N_{\odot}, \mathfrak{F}_{T}, x_{h, h^{\prime}}, D_{+}, m_{+}, T_{\pi,+}$,

A.8 $D_{\Sigma}^{*}, \Sigma^{l}, \mathfrak{a}^{\prime}, \dagger$.

\section{REFERENCES}

[AM] Alekseev, A.Y., Malkin, A.Z., Symplectic structures associated to Lie-Poisson groups, Comm. Math. Phys. 162 (1994), 147-173. MR1272770 (95g:58074)

[AZ] Artin, M., Zhang, J.J., Noncommutative projective schemes, Adv. Math. 109 (1994), 228-287. MR 1304753 (96a:14004)

[APW] Andersen, H. H., Polo, P., Wen, K., Representations of quantum algebras, Invent. Math. 104 (1991), 1-59. MR.1094046 (92e:17011)

[B1] Bourbaki, N., Algèbre commutative, Eléments de mathématiques, Hermann, 1961-1965.

[B2] Bourbaki, N., Algèbre, Eléments de mathématiques, Masson, 1980.

[BFG] Bezrukavnikov, R., Finkelberg, M., Ginzburg, V., Cherednik algebras and Hilbert schemes in characteristic p, Represent. Theory 10 (2006), 254-298 (electronic). MR.2219114 $(2007 \mathrm{j}: 14004)$

[BG1] Brown, K., A., Goodearl, I., Homological aspects of Noetherian PI Hopf algebras and irreducible modules of maximal dimension, J. Algebra 198 (1997), 240-265. MR1482982 (99c:16036)

[BG2] Brown, K., A., Gordon, I., Poisson orders, symplectic reflexion algebras, and representation theory, J. Reine Angew. Math 559 (2003), 193-216. MR1989650(2004i:16025)

[BG3] Brown, K., A., Goodearl, I., Lectures on algebraic quantum groups, Advanced Courses in Mathematrics CRM Barcelona, Birkhäuser, 2002. MR1898492 (2003f:16067)

[BGS] Brown, K., A., Gordon, I., Stafford, $\mathcal{O}_{\epsilon}[G]$ is a free module over $\mathcal{O}[G]$, Preprint math.QA/0007179.

[BK1] Bezrukavnikov, R., Kaledin, D., MacKay equivalence for symplectic quotient singularities, Proc. Steklov Inst. Math. 246 (2004), 13-33. MR2101282 (2006e:14006)

[BK2] Brion, M., Kumar, S., Frobenius splitting methods in geometry and representation theory, Progress in Mathematics, vol. 231, Birkhäuser, 2004. MR2107324 (2005k:14104)

[BK3] Backelin, E., Kremnitzer, K., Quantum flag varieties, equivariant quantum D-modules, and localization of quantum groups, Adv. Math. 203 (2006), no. 2, 408-429. MR.2227727 (2007b:17021)

[BS] Bauman, P., Schmitt, F., Classification of bicovariant differential calculi on quantum groups (a representation theoretic approach), Commun. Math. Phys. 194 (1998), 71-96. MR:1628278 (99f:58012)

[C] Cherednik, I., Double affine Hecke algebras, Cambridge University Press, 2005. MR2133033 (2007e:32012)

[CV] Crawley-Boevey, W., Van Den Bergh, M., Absolutely indecomposable representations and Kac-Moody Lie algebras, Invent. Math. 155 (2004), 537-559. MR2038196 (2004m:17032)

[CH] Crawley-Boevey, W., Holland, M. P., Noncommutative deformations of Kleinian singularities, Duke Math. J.. MR.1620538 (99f:14003)

[D1] Dixmier, J., Enveloping algebras, Graduate Studies in Math., vol. 11, Amer. Math. Soc., 1996. MR.1393197 (97c:17010)

[DM] Donin, J., Mudrov, A., Reflection equation, twist, and equivariant quantization, Isr. J. Math. 136 (2003), 11-28. MR.1998103 (2004g:16046)

[DKP] De Concini, C., Kac, V., Procesi, C., Quantum coadjoint action, J. Amer. Math. Soc. 5 (1992), 151-190. MR.1124981 (93f:17020)

[DL] De Concini, C., Lyubashenko, V., Quantum function algebra at roots of 1, Adv. Math. 108 (1994), 205-262. MR1296515 (95m:17014)

[DP] De Concini, C., Procesi, C., Quantum groups, Lecture Notes in Math., vol. 1565, SpringerVerlag, 1993, pp. 31-140. MR.1288995 (95j:17012)

[E] Eisenbud, D., Commutative algebra with a view toward algebraic geometry, Graduate Texts in Math., vol. 150, Springer-Verlag, 1994. MR1322960(97a:13001) 
[EG] Etingof, P., Ginzburg, V., Symplectic reflection algebras, Calogero-Moser space, and deformed Harish-Chandra homomorphism, Invent. Math. 147 (2002), 243-348. MR.1881922 (2003b:16021)

[EGA3] Grothendieck, A., Étude cohomologique des faisceaux cohérents, Publications Mathématiques de l'IHES, vol. 17, 1963.

[EK] Etingof, P., Kirillov, A., Macdonald polynomials and representations of quantum groups, Math. Res. Letters 1 (1994), 279-296. MR.1302644 (96m:17025)

[FR] Fock, V.V., Rosly, A.A., Poisson structure on moduli of flat connections on Riemann surfaces and the r-matrix, Amer. Math. Soc. Transl. Ser. 21991 (1999). MR.1730456 (2001k:53167)

[G] Gabriel, P., Des catégories Abéliennes, Bulletin de la S.M.F. 90 (1962), 323-448. MR0232821 (38:1144)

[GG] Gan, W.L., Ginzburg, V., Almost-commuting variety, D-modules, and Cherednik algebras, Int. Math. Res. Publ. 2006, 26439, 1-54. MR2210660 (2008c:14062)

[J1] Jantzen, J. C., Representations of algebraic groups (2nd edition), Mathematical Surveys and Monographs, vol. 107, American Mathematical Society, 2003. MR2015057 (2004h:20061)

[J2] Joseph, A., Quantum groups and their primitive ideals, Ergeb. Math. Grenzgeb. (3), vol. 29, Springer-Verlag, 1995. MR1315966 (96d:17015)

[K2] Kirillov, A., Lectures on affine Hecke algebras and Macdonald conjectures, Bull. Amer. Math. Soc. (N.S.) 34 (1997), 251-292. MR.1441642 (99c:17015)

[L1] Lin, Z., Induced representations of Hopf algebras: applications to quantum groups at roots of 1, J. Algebra 154 (1993), 152-187. MR.1201918 (94e:16046)

[L2] Lusztig, G., Introduction to quantum groups, Birkhäuser, 1993. MR.1227098 (94m:17016)

[L3] Lu, J.-H., Momentum mappings and reduction of Poisson actions, Symplectic geometry, groupoids, and integrable systems (Berkeley, 1989), Math. Sci. Res. Inst. Publ., vol. 20, 1991, pp. 209-226. MR.1104930 (92j:58036)

[L4] Lu, J.-H., Moment maps at the quantum level, Commun. Math. Phys. 157 (1993), 389404. MR $1244874(94 \mathrm{~m}: 58096)$

[LR] Lunts, V.A., Rosenberg, A. L., Differential operators on noncommutative rings, Selecta Math. New. Ser. 3 (1997), 335-359. MR1481133 (98k:16038)

[LW] Lu, J.H., Weinstein, A., Poisson Lie groups, dressing transformations, and Bruhat decompositions, J. Differential Geometry 31 (1990), 501-526. MR.1037412 (91c:22012)

[M1] Majid, S., Foundations of quantum group theory, Cambridge University Press, 1995. MR.1381692 (97g:17016)

[M2] Milne, J.-S., Étale cohomology, Princeton University Press, 1980. MR559531 (81j:14002)

[MR] McConnell, J.-C., Robson, J.-C., Noncommutative Noetherian rings, Pure Appl. Math., John Wiley and Sons, 1987. MR934572 (89j:16023)

[N] Nakajima, H., Lectures on Hilbert schemes of points on surfaces, University Lecture Series, vol. 18, Amer. Math. Soc., 1999. MR1711344 (2001b:14007)

[NV1] Nastasescu, C., Van Oystaeyen, F., Graded and filtered rings and modules, Lectures Notes in Math., vol. 758, Springer-Verlag, 1979. MR.551625 (80k:16002)

[NV2] Nastasescu, C., Van Oystaeyen, F., Graded ring theory, North-Holland, 1982. MR676974 (84i:16002)

[P] Paradowski, J., Filtrations of modules over the quantum algebra, Algebraic groups and their generalizations: classical methods (Haboush, W.F., Parshall, B.J., eds.), Proc. Sympos. Pure Math., vol. 56:2, 1994, pp. 93-108. MR1278729 (95c:20060)

[O] Oblomkov, A., Double affine Hecke algebras and Calogero Moser spaces, Int. Math. Res. Not. 18 (2004), 877-912. MR2037756 (2005j:20005)

[R] Ryom-Hansen, S., A q-analogue of Kempf's vanishing theorem, Mosc. Math. J. 3 (2003), 173-187. MR.1996807 (2004e:17014)

[S] Seshadri, C. S., Geometric reductivity over arbitrary base, Adv. Math. 26 (1977), 225-274. MR0466154(57:6035)

[T1] Tanisaki, T., Killing forms, Harish-Chandra isomorphisms, and universal R-matrices for quantum algebras, Int. J. Mod. Phys. A7, Suppl. 1B (1992), 941-961. MR1187582 (93k:17040)

[V] Vasserot, E., On induced and simple modules of double affine Hecke algebras, Duke Math. J. 126 (2005), 251-323. MR2115259 (2006j:20011) 
[VV1] Van den Bergh, M., Van Oystaeyen, F., Lifting maximal orders, Comm. Algebra 17 (1989), 341-349. MR978479 (90a:16006)

[VV2] Varagnolo, M., Vasserot, E., From double affine Hecke algebras to quantum affine Schur algebras, Int. Math. Res. Not. 26 (2004), 1299-1333. MR2046511 (2005c:20009)

[VV3] Varagnolo, M., Vasserot, E., Finite-dimensional representations of DAHA and affine Springer fibers: the spherical case, Duke Math. J. 147 (2009), no. 3, 439-540. MR.2510742 (2010e:20011)

Département de Mathématiques, Université de Cergy-Pontoise, 2 av. A. Chauvin, BP 222, 95302 Cergy-Pontoise Cedex, France

E-mail address: michela.varagnolo@math.u-cergy.fr

Département de Mathématiques, Université Paris 7, 175 rue du Chevaleret, 75013 Paris, France

E-mail address: vasserot@math.jussieu.fr 